\title{
Andreas Trügler
}

\section{OPTICAL PROPERTIES OF METALLIC NANOPARTICLES}

\section{DiSSERTATION}

zur Erlangung des akademischen Grades

Doktor rerum naturalium (Dr. rer. nat.)

Institut für Physik, Fachbereich Theoretische Physik

Karl-Franzens-Universität Graz

Betreuer: Ao.Univ.-Prof. Mag. Dr. Ulrich Hohenester

Begutachter: Univ.-Prof. Mag. Dr. Joachim Krenn

Graz, Juli 2011

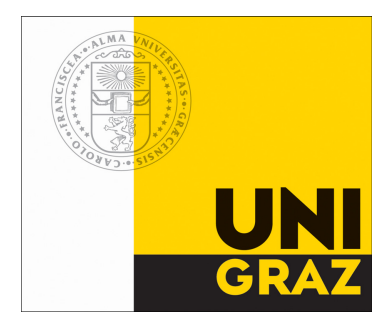





\section{Contents}

Contents

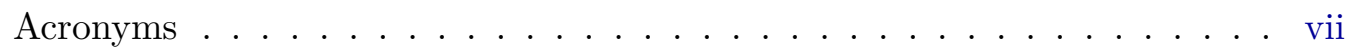

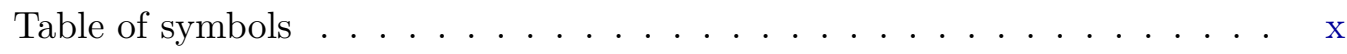

$\begin{array}{lr}\text { I. Introduction and basic principles } & 1\end{array}$

$\begin{array}{ll}\text { 1. Prologue } & 3\end{array}$

1.1. The glamour of plasmonics $\ldots \ldots \ldots \ldots$

1.2. Structure of this thesis . . . . . . . . . . . . 4

1.2.1. Measurement units and material parameter ........ 5

$\begin{array}{lr}\text { 2. The world of plasmons } & 7\end{array}$

2.1. From the first observation to the modern concept of surface plasmons 7

2.2. Derivation of surface plasmon polaritons . . . . . . . . . . . 9

2.2.1. Electromagnetic waves at interfaces . . . . . . . . . . 12

2.2.2. Particle plasmons . . . . . . . . . . . . . . . 17

2.3. Tuning the plasmon resonance . . . . . . . . . . . . . . 18

2.3.1. Principle of plasmonic (bio-)sensing . . . . . . . . . 20

2.3.2. Light absorption in solar cells . . . . . . . . . . . . . . . 25

2.4. Damping mechanisms of surface plasmon polaritons . . . . . . . . 27

2.5. Magnetic effects . . . . . . . . . . . . . . . . . . . 29 
$\begin{array}{ll}\text { 3. Theory } & 31\end{array}$

3.1. Quantum versus classical field theory . . . . . . . . . . . . . . . 31

3.2. Maxwell's theory of electromagnetism . . . . . . . . . . . . 33

3.2.1. Linear and nonlinear optical response . . . . . . . . . . . 35

3.2.2. Nonlocal in space and time . . . . . . . . . . . . 37

3.2.3. Electromagnetic potentials . . . . . . . . . . . . 39

3.3. Rayleigh scattering - The quasistatic approximation . . . . . . . . 42

3.3.1. From boundary integrals to boundary elements . . . . . . . . 44

3.3.2. Eigenmode expansion . . . . . . . . . . . . . . . 45

3.4. Solving the full Maxwell equations . . . . . . . . . . . . . . 50

3.4.1. Boundary conditions . . . . . . . . . . . . . . 52

3.4.2. Surface charges and currents . . . . . . . . . . . 52

4. Modeling the optical response of metallic nanoparticles 55

4.1. Analytic solutions . . . . . . . . . . . . . . . 56

4.1.1. Quasistatic approximation - Rayleigh theory . . . . . . . 56

4.1.2. Mie theory $\ldots \ldots \ldots \ldots \ldots \ldots$

4.1.3. Mie-Gans solution . . . . . . . . . . . . . . . 61

4.2. Discrete Dipole Approximation . . . . . . . . . . . . . . 63

4.2.1. Cross sections with DDA . . . . . . . . 66

4.3. Boundary Element Method . . . . . . . . . . . . . . . 67

4.4. Other methods . . . . . . . . . . . . . . . . . . 69

4.5. Comparison between different approaches . . . . . . . . . . . 70

4.5.1. Accuracy . . . . . . . . . . . . . . 70

4.5.2. Performance .................... . . 71

4.5.3. Limits and inaccuracies . . . . . . . . . . 73 
5. Imaging of surface plasmons

5.1. Principles of near-field optics . . . . . . . . . . . . 75

5.2. How to picture a plasmon . . . . . . . . . . . . 78

5.2.1. Mapping the plasmonic LDOS . . . . . . . . . . 78

5.2.2. Electron energy loss spectroscopy . . . . . . . . . . . . . . . 78

6. Influence of surface roughness 85

6.1. Generation of a rough particle in the simulation . . . . . . . . . 85

6.2. Theoretical analysis of surface roughness . . . . . . . . . . . . . . 89

7. Nonlinear optical effects of plasmonic nanoparticles 91

7.1. Autocorrelation . . . . . . . . . . . . . . . . . 93

7.2. Third harmonic imaging . . . . . . . . . . . . . . . . . . 94

$\begin{array}{ll}\text { II. Paper reprints } & 97\end{array}$

8. Optimal aspect ratio for Bio-sensing, Plasmonics 5, 161 (2010) 101

8.1. Introduction . . . . . . . . . . . . . . . . . . . . . . . . 102

8.2. Plasmon sensor quality . . . . . . . . . . . . . . . 103

8.3. Results. . . . . . . . . . . . . . . . . 105

8.4. Discussion . . . . . . . . . . . . . . . 106

8.5. Conclusion . . . . . . . . . . . . . . . . . 110

8.5.1. Acknowledgment . . . . . . . . . . . . . . . . . 111

8.6. Appendix - Methods . . . . . . . . . . . . . . . . . . . 111

8.6.1. Simulations based on boundary element method . . . . . . . 111

8.6.2. Determination of $S, \mathrm{FOM} \mathrm{FOM}^{*}$, and $\mathrm{FOM}_{\text {layer }}^{*} \ldots \ldots . . .112$

8.6.3. Gold nanorods preparation . . . . . . . . . . . . . . 112

8.6.4. Single particle spectroscopy . . . . . . . . . . . . . . 112 
$\begin{array}{ll}\text { 9. Highly sensitive silver nanorods, submitted (2011) } & 115\end{array}$

9.1. Introduction . . . . . . . . . . . . . . . 116

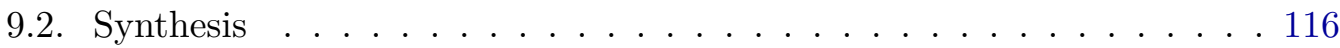

9.3. Plasmonic nanorod sensitivity . . . . . . . . . . . . . . 117

9.4. Sensitivity measurements _ . . . . . . . . . . . . . . . 119

9.5. Discussion and Model . . . . . . . . . . . . . . . . . . . 121

9.6. Sensing Reversibility . . . . . . . . . . . . . . . . . . . 122

9.7. Conclusion . . . . . . . . . . . . . . . . . 122

9.8. Methods . . . . . . . . . . . . . . . . . . . . 123

9.8.1. Reagents . . . . . . . . . . . . . . . . 123

9.8.2. Synthesis of Silver Nanorods . . . . . . . . . . . . . . . . 124

9.8.3. Characterization . . . . . . . . . . . . . . . 124

9.9. Supporting Material . . . . . . . . . . . . . . . . 125

9.9.1. Derivation of the plasmonic sensitivity equation . . . . . . . . 127

9.10. Acknowledgments . . . . . . . . . . . . . . . . . . . 128

10. Superresolution Moiré mapping, PRL 104, 143901 (2010) 129

11. High-resolution plasmon imaging, PRB 79, 041401(R) (2009) 139

11.1. Introduction . . . . . . . . . . . . . . . . . . . 140

11.2. Experiment . . . . . . . . . . . . . . . . 141

11.3. Theory . . . . . . . . . . . . . . . . . . . . . . 143

11.4. Discussion . . . . . . . . . . . . . . . . . . . . 145 
12. Influence of surface roughness, PRB 83, 081412(R) (2011) 149

12.1. Introduction . . . . . . . . . . . . . . . . . . . . . 150

12.2. Experiment . . . . . . . . . . . . . . . . . . 151

12.3. Simulation . . . . . . . . . . . . . . . . . . . . . 151

12.4. Theory . . . . . . . . . . . . . . . . . 155

12.5. Summary . . . . . . . . . . . . . . . . . . 158

13. Light confinement in nanoantennas, submitted (2011) 159

13.1. Light confinement in nanoantennas . . . . . . . . . . . . . . . . . 160

13.2. Supplementary Information . . . . . . . . . . . . . . . . . . . 169

$\begin{array}{ll}\text { III. Résumé } & 173\end{array}$

$\begin{array}{lr}\text { 14. Summary } & 175\end{array}$

14.1. What has been done . . . . . . . . . . . . . . . . . . 175

14.2. What is left to do . . . . . . . . . . . . . . . . 175

$\begin{array}{lr}\text { IV. Supplement } & 179\end{array}$

$\begin{array}{ll}\text { A. Appendix - Utilities } & 181\end{array}$

A.1. Boundary conditions at different media . . . . . . . . . . . . . . 181

A.2. Conversion between $\mathrm{nm}$ and $\mathrm{eV} \ldots \ldots$. . . . . . . . . . . . 184

A.3. Conversion between FWHM and decay time . . . . . . . . . . 185

A.4. Derivation of retarded surface charges and currents . . . . . . . . 187

A.5. Spherical harmonics . . . . . . . . . . . . . . . . 188

A.5.1. Completeness and normalisation . . . . . . . . . . . 190

A.5.2. Expansions in series of the spherical harmonics . . . . . . . . . 190

A.6. Generating functions for the vector harmonics . . . . . . . . . . . . 192 
$\begin{array}{ll}\text { B. Appendix - Fabrication methods } & 193\end{array}$

B.1. Chemical synthesis . . . . . . . . . . . . . . . . . . . . 193

B.2. Electron beam lithography . . . . . . . . . . . . . . . . . . . . 194

$\begin{array}{lr}\text { C. Appendix - MATLAB } & 197\end{array}$

C.1. MATLAB memory limitations for different operating systems . . . . . 197

C.2. MATLAB script for Mie solution . . . . . . . . . . . . . . . . . . 199

$\begin{array}{ll}\text { Bibliography } & 203\end{array}$

List of Equations 223

$\begin{array}{ll}\text { List of Figures } & 225\end{array}$

$\begin{array}{ll}\text { List of Tables } & 229\end{array}$

$\begin{array}{lr}\text { Index } & 230\end{array}$ 


\section{Acronyms}

ABC absorbing boundary conditions 72

AR aspect ratio 103, 105-110, 117, 127

BEM Boundary Element Method 44, 50, 52, 67, 68, 70$73,80,88,95,103,105-107,109,110,115,119$ $121,126,134,135,137,143,144,151-153,155$, $157,164,166,175$

CTAB cetyl trimethylammonium bromide 120

DDA Discrete Dipole Approximation 63, 64, 70-72, 175

DFG Deutsche Forschungsgemeinschaft 111, 128

DNA deoxyribonucleic acid 3

EELS Electron Energy Loss Spectroscopy 7, 78-83, 140$147,175,176$

EFTEM energy-filtered transmission electron microscopy 81, 141-147

EIT electromagnetically induced transparency 104, 111

FDTD Finite Difference Time Domain 69, 72

FOM 'figure of merit' 103-108, 110-112, 119

FWF Austrian science fund 147, 158

FWHM full width at half maximum 27, 28, 116, 165, 171, $185,186,195$

GASS Graz Advanced School of Science 111, 128, 138

ITO indium-tin oxide 151, 194 
LDOS local density of states $78,81,82,130,132,134,136$, 138,175

LED light emitting diode 116, 126

M $\quad$ Mol 118, 122, 124

MNP metallic nanoparticle 4, 5, 20, 21, 25, 55, 79, 85, 91

MoM Method of Moments 69

OS $\quad$ operating system 198

PET polyethylene terephthalate 112

PMMA poly(methyl methacrylate) 195

PtTFPP platinum(II)-5,10,15,20-tetrakis-(2,3,4,5,6pentafluorphenyl)-porphyrin 131-134, 136-138

PVP polyvinylpyrrolidone 120, 123, 124

QED quantum electrodynamics 31,32

QSA quasi-static approximation 105-107

RIU refractive index unit 103, 120

SEM scanning electron microscope 88, 92, 151, 152, 162, 172

SHG second-harmonic generation 91-93

SNOM scanning near-field optical microscopy 30,78

SPP surface plasmon polariton 16

STD standard deviation 125

STEM scanning transmission electron microscopy 81, 140, $141,143,146$

SU8 epoxy resin 131-134, 136-138

TE transverse electric 13,14 
TEM transmission electron microscopy 119, 124, 125, 141,142

THG third-harmonic generation 92-95, 161, 162, 164, 165,172

TM transverse magnetic 13

ZLP $\quad$ zero-loss peak 140, 145, 146 


\section{Table of symbols}

$\tilde{\boldsymbol{A}} \quad$ Complex $3 N \times 3 N$ matrix 64,65

$\boldsymbol{A} \quad$ Vector potential of Maxwell's theory 40, 50

$\boldsymbol{B} \quad$ Magnetic field 30, 33, 182

$C_{\text {abs }} \quad$ Absorption cross section 66

$C_{\text {ext }} \quad$ Extinction cross section 66

$C_{\text {sca }} \quad$ Scattering cross section $66,112,127$

$\Delta h \quad$ Scaling of stochastic height variations $h 86,87,154$, 155

$\Delta k_{x} \quad$ Uncertainty of the momentum of a microscopic particle in the spatial $x$-direction $75-77$

$\Delta x \quad$ Uncertainty in the spatial position of a microscopic particle 75

D Dielectric displacement 33, 56, 182

$E_{F} \quad$ Fermi energy 29

$\tilde{\boldsymbol{E}} 3 N$-dimensional (complex) vector of the electric field at each lattice site 64,65

$E_{\text {res }} \quad$ Resonance energy 103, 108

$\boldsymbol{E} \quad$ Electric field 9, 23, 30, 33, 35-39, 56, 64, 65, 92, 183,185

$\boldsymbol{F}_{L} \quad$ Lorentz force 29

$\boldsymbol{F} \quad$ Surface derivative of Green function $G$ expressed as a matrix 45,46

$\mathcal{F}^{-1} \quad$ Inverse Fourier transform 38, 87, 153

$\mathcal{F} \quad$ Fourier transform 38

$F \quad$ Surface derivative of Green function $G 44,45,51$, $89,156,157$

$G_{2} \quad$ Second order autocorrelation function 93

$G_{3} \quad$ Third order autocorrelation function 93

$\Gamma \quad$ Homogeneous linewidth (FWHM) of a spectral resonance $27,28,103,106,107,110,166,185$

$\mathcal{G} \quad$ Grating constant 130-133, 135, 137 
$G \quad$ Green function, same symbol for quasistatic and retarded case 42-44, 50, 51, 155, 156

$\boldsymbol{H} \quad$ Magnetic field 33

$\boldsymbol{\Lambda}$ Matrix containing the dielectric information of a quasistatic problem 45, 47, 156, 157

$L_{i} \quad$ Geometrical shape factor for ellipsoidal particles needed for the Mie-Gans solution 62

$L \quad$ Shape factor, characteristic length scale of a structure $75,107,127,128$

$\mathcal{M}$ Vector harmonic, $\mathcal{M}=\boldsymbol{\nabla} \times(\boldsymbol{r} \psi) 57,58,192$

$\boldsymbol{M} \quad$ Magnetic moment per unit volume 35

NA Numerical aperture of an optical system 75, 161163

$\mathcal{N} \quad$ Vector harmonic, $\mathcal{N}=\frac{1}{k} \boldsymbol{\nabla} \times \mathcal{M} 57,58$

$N_{\omega} \quad$ Number of calculated frequencies 72

$N \quad$ Normalization factor $(N \in \mathbb{R})$ or total number of entities $(N \in \mathbb{N})$, e.g. lattice sites or number of phases $63-65,86,87$

$\Omega \quad$ Boundary region of a metallic nanoparticle or sphere segment 136

$\tilde{\boldsymbol{P}} \quad 3 N$-dimensional (complex) vector of the dipole polarization at each lattice site $64-66$

$\Phi \quad$ Scalar potential 40, 42, 43, 155, 182

$P_{\alpha}^{(L)} \quad \alpha$ th component of the linear dipole moment per unit volume, $\alpha \in\{x, y, z\} 37,39$

$P_{l}^{m} \quad$ Associated Legendre functions of the first kind of degree $l$ and order $m 58$

$\boldsymbol{P} \quad$ Dipole moment per unit volume (polarization) $35-$ $39,56,64$

Q Quality factor 109, 110

$R \quad$ Radius of a sphere 44 
$S \quad$ Sensitivity, often simply denoted as $\Delta \lambda /$ RIU, can be expressed in wavelength $\left(S_{\lambda}\right)$ or energy $\left(S_{E}\right)$ units 103, 105-111, 119-121

$T_{2} \quad$ Decoherence time 167

$T \quad$ Time constant 93

$V \quad$ Volume of a nanoparticle 56, 72, 107, 181

$\boldsymbol{\alpha} \quad$ Polarizability tensor 64

$\alpha \quad$ Polarizability, e.g. of a spheroid 56, 61, 62, 107, 127

$a_{l} \quad$ Mie scattering coefficient for mode $l 60$

$b_{l} \quad$ Mie scattering coefficient for mode $l 60$

$\chi_{e} \quad$ Electric susceptibility (also see $\chi$ ) 37

$\chi^{(i)} \quad$ Susceptibility tensor of rank $(i+1) 36-38,92$

$\chi_{m} \quad$ Magnetic susceptibility (also see $\boldsymbol{\chi}$ ) 29

$c_{l} \quad$ Mie coefficient for inside field and mode $l 60$

c Speed of light, in vacuum: $299792458 \mathrm{~m} / \mathrm{s}$ 15, 29, $33,75,121,127,184$

$\delta F \quad$ Small deviation of $F$ due to surface roughness 89 , 156, 157

$\partial \Omega \quad$ Boundary of a region $\Omega$, e.g. the surface of a nanoparticle 43, 44, 89, 155, 156, 181, 182

$\delta \quad$ Plasmon propagation length 17

$d_{l} \quad$ Mie coefficient for inside field and mode $l 60$

$\boldsymbol{d}$ Dipole moment of a two level system, e.g. a molecule 135

$d \Omega \quad$ Element of the solid angle, in spherical polar coordinates: $d \Omega=\sin (\theta) d \theta d \varphi 66$

$\hat{e} \quad$ Unit vector, for example along the axis of an orthogonal coordinate system 40, 44, 62

$e \quad$ Electron charge 9

$\gamma \quad$ Decay rate $9,10,127,135,136$

$\hbar \quad$ Reduced Planck constant or Dirac constant 75, 135, 184, 185, 189 
$h \quad$ Stochastic height variations to model surface roughness $85,87,89,153$

$j \quad$ Subscript index referring to a dielectric medium $j$ or a general counting index, $j \in \mathbb{N} 51$

$\boldsymbol{j} \quad$ Electromagnetic current density 33, 34, 64, 65, 182

$\boldsymbol{k} \quad$ Wave vector of light $13-17,40,75,83,89$

$k_{j} \quad$ Wave number in medium $j 51$

$\tilde{k}$ Imaginary part of complex refractive index $n=$ $\tilde{n}+i \tilde{k} 11,199$

$k \quad$ Wave number in vacuum $40,42,50,66,75,76$

$\lambda_{k} \quad$ Eigenenergy of mode $k$ of matrix $\boldsymbol{F} 47,49,89,156$, 157

$\lambda \quad$ Wavelength of light $16,17,42,63,66,75,76,103-$ $106,112,119,121,123,124,126-128,185$

$\triangle \quad$ Laplace operator 188

$l \quad$ Thickness of a layer of homogeneous coating around a nanoparticle 104, 105, 107, 112

$m_{e} \quad$ Mass of the free electron 9

$m_{\gamma} \quad$ Photon mass $\left(m_{\gamma}<4 \times 10^{-51} \mathrm{~kg}\right) 34$

$\mu \quad$ Magnetic permeability, $\mu=1$ for optical wavelengths 29, 32, 33, 60

$n_{b} \quad$ Background refractive index, usually $n_{b} \in \mathbb{R}^{+} 53$, 68, 109, 113, 119, 121, 127, 128, 151, 154

$n_{e} \quad$ Electron density 10

$\hat{\boldsymbol{n}} \quad$ Unit vector (for example pointing in the scattering direction) 44, 66, 181

$\tilde{n} \quad$ Real part of complex refractive index $n=\tilde{n}+i \tilde{k}$ 11, 199

$n \quad$ Refractive index of a medium, for metals $n \in \mathbb{C} 10$, 11, 15, 26, 60, 103, 104, 106, 107, 112, 119, 121, $123,128,199$

$\omega_{p} \quad$ (Bulk) plasma frequency of the conduction electrons of a metal (9 eV for gold) 10, 109, 121, 127 
$\omega \quad$ Angular frequency of a photon 9, 10, 14, 15, 40, 47, $61,64,72,73,75,89,91,127,135,144,155-157$, 185,186

$\phi_{\text {rnd }} \quad$ Stochastic phase factors 87, 153

$\phi_{\text {ext }}$ Potential of an external excitation like a plane wave or a dipole $43,155,156$

$\psi \quad$ Generating function for the vector harmonics $\mathcal{N}$ and $\mathcal{M}, \psi=\psi(r, \theta, \varphi) 57,58$

$p \quad$ Norm of the momentum $\boldsymbol{p}$ of a particle 75

$q \quad$ Electromagnetic charge 29

$\boldsymbol{r} \quad$ Spatial position vector, $\boldsymbol{r} \in \mathbb{R}^{3} 9,35-38,40,43,44$, 73, 135, 136, 155

$\rho \quad$ Radius in a polar coordinate system 44

$r_{s} \quad$ Electron gas parameter 10

$r \quad$ Norm of spatial position vector, radial component of spherical polar coordinates 57, 80, 188

$s \quad$ Spatial position vector on a surface $\partial \Omega 43-45,155$, 156

$\sigma_{k}^{L} \quad$ Left eigenvector of $\boldsymbol{F}$, can be interpreted as the $k^{\text {th }}$ surface plasmon eigenmode 46, 49, 156

$\sigma_{k}^{R} \quad$ Right eigenvector of $\boldsymbol{F}$, can be interpreted as the $k^{\text {th }}$ surface plasmon eigenmode 46,156

$\sigma \quad$ Surface charge distribution 43-45, 49, 51, 53, 154, 155, 181-183

$\tau$ Dephasing time of a surface plasmon polariton, typically $\tau<10$ fs. Further 27, 28, 161, 162, 166, $167,185,186$

$\hat{\boldsymbol{t}} \quad$ Tangential unit vector 181

$\theta \quad$ Inclination angle of spherical polar coordinates 57, 58,188

$t \quad$ Time variable, $t \in \mathbb{R}$ 35-38, 40, 93, 185

$v_{F} \quad$ Fermi velocity 29 
$\varepsilon \quad$ Dielectric description of a material, may be a constant or a frequency dependent (complex) function $10,11,13,16,17,19,20,32,33,45,56,61,70,71$, 73, 89, 107, 109, 121, 127, 128, 155, 199

$\varphi \quad$ Angle in a polar coordinate system 44, 57, 188

$\varrho \quad$ External or free charge distribution of Maxwell's theory 33-36, 42, 80, 182

$\varsigma \quad$ Variance of height fluctuations in a Gaussian autocorrelation function 85, 87, 153, 154

$\boldsymbol{v} \quad$ Velocity of a point charge 29

$\zeta \quad$ Skin depth of evanescent field 16, 17, 72

$z_{l} \quad$ Substitute for any of the four spherical Bessel functions $j_{l}, y_{l}, h_{l}^{(1)}$, or $h_{l}^{(2)} 58$ 

Dedicated to my parents. 



\section{Part I.}

\section{Introduction and basic principles}





\section{Prologue}

A lot has happened since the struggle between the devotees of the undulatory and corpuscular theory of light. For millennia we have been fascinated by optical phenomena and the groundbreaking works of many brilliant scientists allowed deep insights into the question of what holds the world together in its inmost folds. Step by step we gain more understanding of what light actually is and especially the interaction of light with matter is a treasure trove for new applications and a demanding criterion for the underlying physical theories.

\subsection{The glamour of plasmonics}

Half a century ago, Richard Feynman was already aware of the fact that there is plenty of room at the bottom [1] and he invited his listeners to open up a new field of physics. In the 1970s the term nanotechnology was formed [2,3] and remarkable progress and new discoveries in the "nanoworld" followed - often resulting in a Nobel prize for the respective scientists ${ }^{1}$.

The study of optical phenomena related to the electromagnetic response of metals, which is the topic of this work, led to the development of an emerging and fast growing research field called plasmonics [4] - named after the electron density waves that propagate along the interface of a metal and a dielectric like the ripples that spread across a water surface after throwing a stone into the water [5].

Particularly the enormous progress in the fabrication and manipulation methods of nanometer-sized objects in the last decades allowed us to enter into the fascinating world at the length scale of molecules and DNA strands. There are certain promises of

\footnotetext{
${ }^{1}$ E.g. the invention of the scanning tunneling microscope, the discovery of fullerenes, etc.
} 
plasmonics [5] that are responsible for the current boom in this research field, like the prediction of new superfast computer chips [5], new possibilities to treat cancer [6], ultrasensitive molecular detectors [7-9] or the ability of making things invisible with negative-refraction materials [10-12]. All this is possible because plasmonics builds a bridge between two different length scales by confining light on sub-wavelength volumes. The building bricks of this arch are metallic nanoparticles (MNPs) and colloids, or thin metal films in the case of plasmonic waveguides. The focus of this work lies on the description of metallic nanoparticles - their optical properties, how they influence and interact with their surrounding, and how we can make these events visible although the involved structural sizes are much below the wavelength of light. Besides the already mentioned, the capability to manipulate and control light on the nanometer scale opens up a plethora of further possible applications, as diverse as data storage [13], optical data processing [14,15], quantum optics [16,17], optoelectronics $[18,19]$, photovoltaics [20], or quantum information processing.

\subsection{Structure of this thesis}

This thesis is divided into three main parts. The first one aims at providing a general introduction to the topic as well as a detailed description of the underlying theoretical concepts. At the beginning of Chap. 2 a short historical overview of plasmons is given - from their first observation to their modern perception. This synopsis is then followed by a sound discussion of surface plasmon polaritons and the rest of the chapter is dedicated to different plasmonic properties and how they can be engineered and exploited. Chap. 3 represents the formal part of this dissertation where the mathematical description of metallic nanoparticles is formulated. In Chap. 4 this theoretical part is then followed by the introduction and discussion of different numerical approaches to solve the previous derivated equations. Chap. 5 is dedicated to the question of how to picture a plasmon, Chap. 6 and Chap. 7 cover the influence of surface roughness and nonlinear optical effects of metallic nanoparticles, respectively.

The second main part consists of a reprint of six papers, that were elaborated during the last years and that represent the essential outcome of the research activity of 
the author. Four of these papers have already been published in high quality peerreviewed journals like Physical Review Letters or Physical Review B. The other two have been submitted and are being reviewed.

After a brief conclusion, the last part of this work contains supplementary material like appendices, a list of figures and tables as well as a cross-reference index. For quick reference several important relations that are highlighted by a box throughout this thesis are collected in the list of equations. App. A contains additional derivations and explanations and App. B provides a short overview about fabrication methods of MNPs. Finally, in App. C some details about the simulation software MATLAB ${ }^{\circledR}$ is given.

A comprehensive glossary is given at the beginning of this document, containing a list of all important symbols and acronyms. To make work easier with the cited papers and books, they have been cross-referenced with online links; the symbol in the bibliography constitutes a direct link to pdf-files whenever possible.

\subsubsection{Measurement units and material parameter}

We are use Gaussian units throughout. The quantities plotted in the figures are almost exclusively expressed in femtoseconds [fs], electron Volts $[\mathrm{eV}]$ or nanometers $[\mathrm{nm}]$. Unless indicated otherwise, the considered metallic nanoparticles are solely made of gold $(\mathrm{Au})$ or silver $(\mathrm{Ag})$. 



\section{The world of plasmons}

Many of the fundamental electronic properties of the solid state can be described by the concept of single electrons moving between an ion lattice. If we ignore the lattice, in a first approximation, we end up with a different approach where the free electrons of a metal can be treated as an electron liquid of high density [21,22]. From this plasma model it follows that longitudinal density fluctuations, so called plasma oscillations or Langmuir waves, with an energy of the order of $10 \mathrm{eV}$ will propagate through the volume of the metal. These volume excitations have been studied in detail with Electron Energy Loss Spectroscopy (EELS) ${ }^{1}$ and have led to the discovery of surface plasmon polaritons.

\subsection{From the first observation to the modern concept of surface plasmons}

The first documented observation of surface plasmon polaritons dates back to 1902, when Wood ${ }^{2}$ illuminated a metallic diffraction grating with polychromatic light and noticed narrow dark bands in the spectrum of the diffracted light, which he referred to as anomalies $[24,25]$.

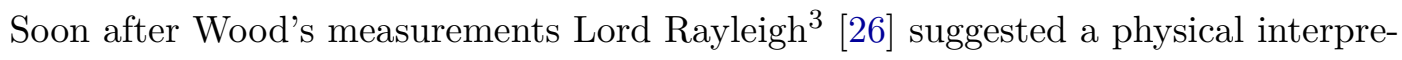
tation of the phenomenon [27], but nevertheless it took several years until $\mathrm{Fano}^{4}$ [28] associated these anomalies with the excitation of electromagnetic surface waves on

\footnotetext{
${ }^{1}$ A historical overview of electron beam experiments to study surface plasmons can be found in [23], page 47.

${ }^{2}$ Born $2^{\text {nd }}$ May 1868 in Concord, Massachusetts; $\dagger 11^{\text {th }}$ August 1955 in Amityville, New York

${ }^{3}$ Born $12^{\text {th }}$ November 1842 in Langford Grove, Essex; $\dagger 30^{\text {th }}$ June 1919 in Witham, Essex.

${ }^{4}$ Born $28^{\text {th }}$ July 1912 in Turin; $\dagger 13^{\text {th }}$ February 2001 in Chicago.
} 
the diffraction grating. In 1957 Ritchie proposed the concept of surface plasmons in the context of electron energy loss in thin films [29] and the experimental verification followed two years later by Powell and Swan [30,31].

In 1958 experiments with metal films on a substrate [32] again showed a large drop in optical reflectivity, and ten years later the explanation and repeated optical excitation of surface plasmons were reported almost simultaneously by Otto [33] as well as Kretschmann and Raether [34]. They established a convenient method for the excitation of surface plasmons [24].

The principle of the plasmon excitation by Otto and Kretschmann is shown in Fig. 2.6 and discussed in the caption. 


\subsection{Derivation of surface plasmon polaritons}

Plasmons are (bosonic) elementary excitations in a metallic ${ }^{5}$ solid. The question whether we should treat them in a quantum mechanical or classical way is discussed in Sec. 3.1.

One of the most simple but nevertheless very utile models to describe the response of a metallic particle exposed to an electromagnetic field was proposed by Paul Drude ${ }^{6}$ $[37,38]$ at the beginning of the 20th century and further extended by Hendrik Lorentz ${ }^{7}$ five years later (consult [39] for a detailed discussion). In 1933 Arnold Sommerfeld ${ }^{8}$ and Hans Bethe ${ }^{9}$ expanded the classical Lorentz-Drude model and eliminated some problems in the description of thermal electrons by accounting for the Pauli principle of quantum mechanics and replacing the Maxwell-Boltzmann with the Fermi-Dirac distribution (again see [39]).

Drude assumed a microscopic description of the electron dynamics in a metal in classical terms, and obtained the equation of motion of a damped oscillator where the electrons are moving between heavier, relatively immobile background ions:

\section{Drude-Sommerfeld model of a free electron gas}

$$
m_{e} \frac{\partial^{2} \boldsymbol{r}}{\partial t^{2}}+m_{e} \gamma_{d} \frac{\partial \boldsymbol{r}}{\partial t}=e \boldsymbol{E}_{0} e^{-i \omega t}
$$

where $\gamma_{d}$ describes a phenomenological damping term, $m_{e}$ the effective free electron mass, $e$ the free electron charge and $\omega$ and $\boldsymbol{E}_{0}$ are the frequency and amplitude of the applied electric field respectively. Eq. (2.1) can be solved by the ansatz $\boldsymbol{r}(t)=\boldsymbol{r}_{0} e^{-i \omega t}$ [4] which directly leads to the dielectric function of Drude form

\footnotetext{
${ }^{5}$ Recently it has been shown [35,36] that also doped graphene may serve as an unique twodimensional plasmonic material with certain advantages compared to metals (lower losses and much longer plasmon lifetimes).

${ }^{6}$ Born $12^{\text {th }}$ July 1863 in Braunschweig; $\dagger 5^{\text {th }}$ July 1906 in Berlin.

${ }^{7}$ Born $18^{\text {th }}$ July 1853 in Arnheim; $\dagger 4^{\text {th }}$ February 1928 in Haarlem.

${ }^{8}$ Born $5^{\text {th }}$ December 1868 in Königsberg; $\dagger 26^{\text {th }}$ April 1951 in München.

${ }^{9}$ Born $2^{\text {th }}$ July 1906 in Straßburg; $\dagger 6^{\text {th }}$ March 2005 in Ithaca, New York.
} 


\section{Dielectric function of Drude form}

$$
\varepsilon_{d}(\omega)=\varepsilon_{\infty}-\frac{\omega_{p}^{2}}{\omega^{2}+i \gamma_{d} \omega}, \quad \text { with } \quad \omega_{p}=\sqrt{\frac{4 \pi n_{e} e^{2}}{m_{e}}}
$$

Here $\omega_{p}$ is the volume or bulk plasma frequency (electron density $n_{e}=3 /\left(4 \pi r_{s}{ }^{3}\right), r_{s}$ is the electron gas parameter and takes the value $0.16 \mathrm{~nm}$ for gold and silver [39]) and $\varepsilon_{\infty}$ describes the ionic background in the metal. If we neglect $\gamma_{d}$ and $\varepsilon_{\infty}$ for the moment, the Drude dielectric function simplifies to $\varepsilon_{d}=1-\omega_{p}^{2} / \omega^{2}$ and we can distinguish two frequency regions: If $\omega$ is larger than $\omega_{p}, \varepsilon_{d}$ is positive and the corresponding refractive index $n=\sqrt{\varepsilon_{d}}$ is a real quantity ${ }^{10}$. But if $\omega$ is smaller than $\omega_{p}, \varepsilon_{d}$ becomes negative and $n$ is imaginary. An imaginary refractive index implies that an electromagnetic wave cannot propagate inside the medium.

The specific value of $\omega_{p}$ for most metals lies in the ultraviolet region, which is the reason why they are shiny and glittering in the visible spectrum. A light wave with $\omega<\omega_{p}$ is reflected, because the electrons in the metal screen the light. On the other hand if $\omega>\omega_{p}$ the light wave gets transmitted (the metal becomes transparent), since the electrons in the metal are too slow and cannot respond fast enough to screen the field.

This treatment of a free electron gas already gives quite accurate results for the optical properties of metals in the infrared region, but since higher-energy photons can also promote bound electrons from lower-lying bands into the conduction band [4] (see Fig. 2.1) the Drude model becomes inaccurate for the visible regime as indicated in Fig. 2.2.

As will be discussed in Sec. 3.1 in more detail, the dielectric function describes the response of a material and can either be obtained by first principle calculations or

\footnotetext{
${ }^{10}$ In general $n= \pm \sqrt{\varepsilon}$ and the positive sign is chosen for causality reasons in the system. A negative refractive index does not occur in nature but can be artificially generated with metamaterials, e.g. see $[10-12]$.
} 

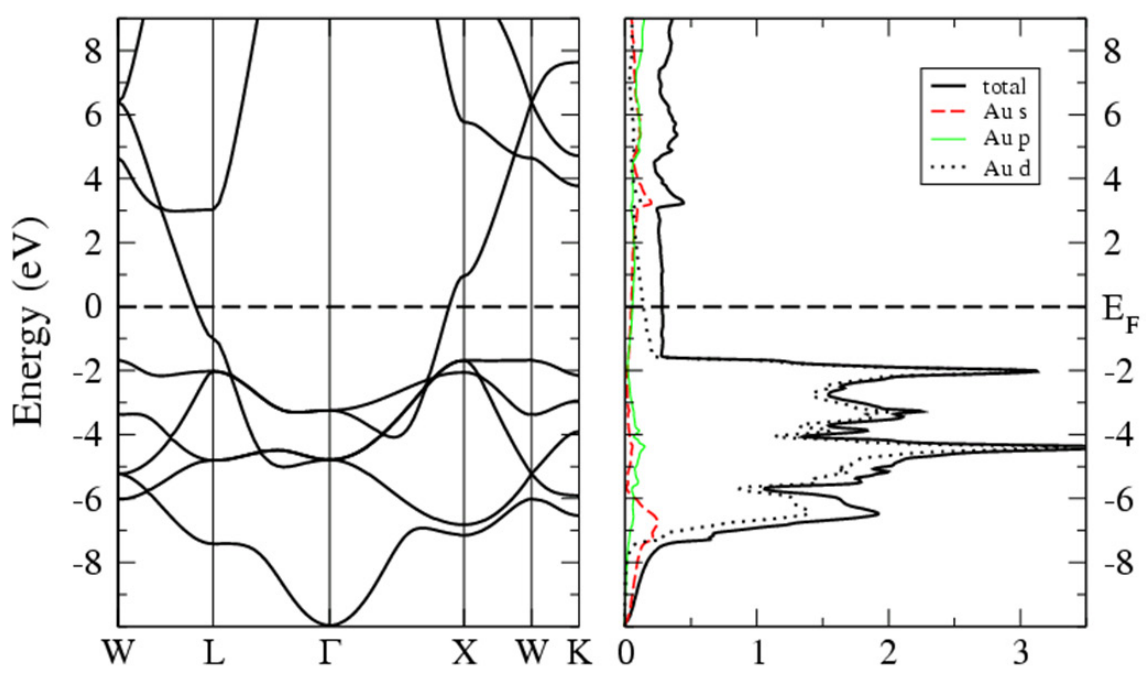

Figure 2.1.: Electronic band structure of gold, taken from [40]. Above $2 \mathrm{eV}$ (i.e. light wavelengths shorter than $620 \mathrm{~nm}$ ) electrons can be promoted from the d-bands below the Fermi energy to states above, which leads to strong plasmon damping and the absorption of bluish light yielding the golden color.

from measurements. In our simulations we will use the dielectric data ${ }^{11}$ obtained from experiments, but unfortunately there are some difficulties. First the results from different experiments are not always consistent as shown in Fig. 2.3. Especially the data published in [43] shows some additional features compared to [41] for gold and silver around $1 \mathrm{eV}(\approx 1240 \mathrm{~nm})^{12}$, see real part in Fig. 2.3(a) and zig-zag features in Fig. 2.3(b). Second the dielectric function can be determined from optical experiments on bulk solids, thin solid films or clusters [44].

\footnotetext{
${ }^{11}$ In the literature most of the time the complex refractive index is tabulated, the connection to the dielectric function is given by $\varepsilon=\varepsilon_{1}+i \varepsilon_{2}=n^{2}=(\tilde{n}+i \tilde{k})^{2}$. The real and imaginary parts of $\varepsilon$ then follow as $\varepsilon_{1}=\tilde{n}^{2}-\tilde{k}^{2}$ and $\varepsilon_{2}=2 \tilde{n} \tilde{k}$.

${ }^{12}$ The conversion factor between $\mathrm{eV}$ and $\mathrm{nm}$ is 1239.84 as discussed in App. A.2.
} 

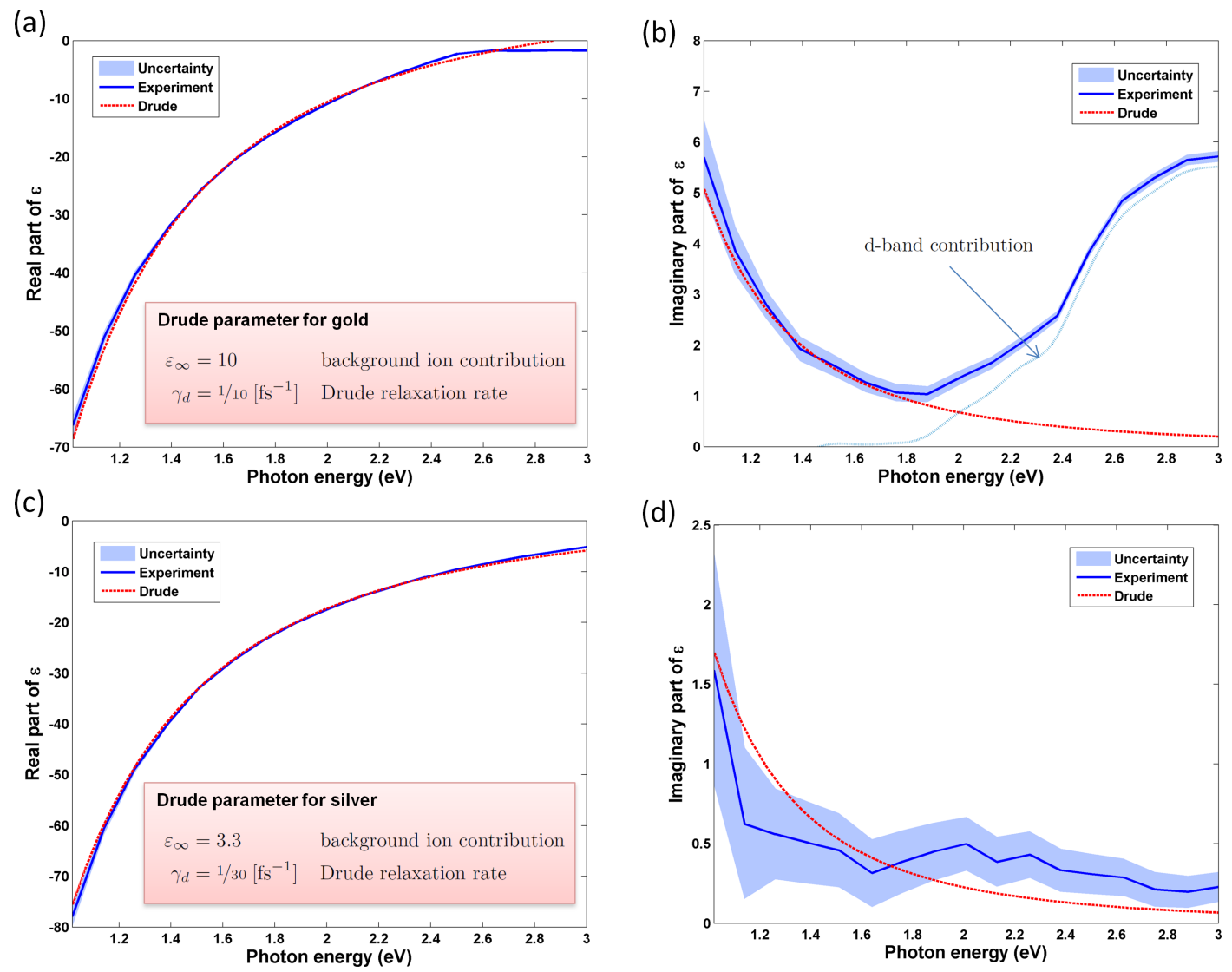

Figure 2.2.: Real and imaginary parts of the dielectric function for gold (a)-(b) and silver (c)-(d). The experimental data together with the measuring uncertainty have been taken from Johnson and Christy [41]. In the inset on the left hand panels we list the corresponding Drude parameters, see Eq. (2.2). The imaginary part of the Drude dielectric function for gold becomes invalid for energies above $1.9 \mathrm{eV}$, see (b), because at this energy interband transitions set in. The line for the dband contribution in (b) is obtained from a simple comparison between the Drude dielectric function and the experimental result, also see [42].

\subsubsection{Electromagnetic waves at interfaces}

In Chap. 3 we will see that the wave equation (3.16) in Helmholtz form is the one relation to rule them all, electromagnetic fields always have to obey:

$$
\left(\nabla^{2}+\varepsilon k^{2}\right) \boldsymbol{E}(\boldsymbol{r}, \omega)=\mathbf{0} .
$$



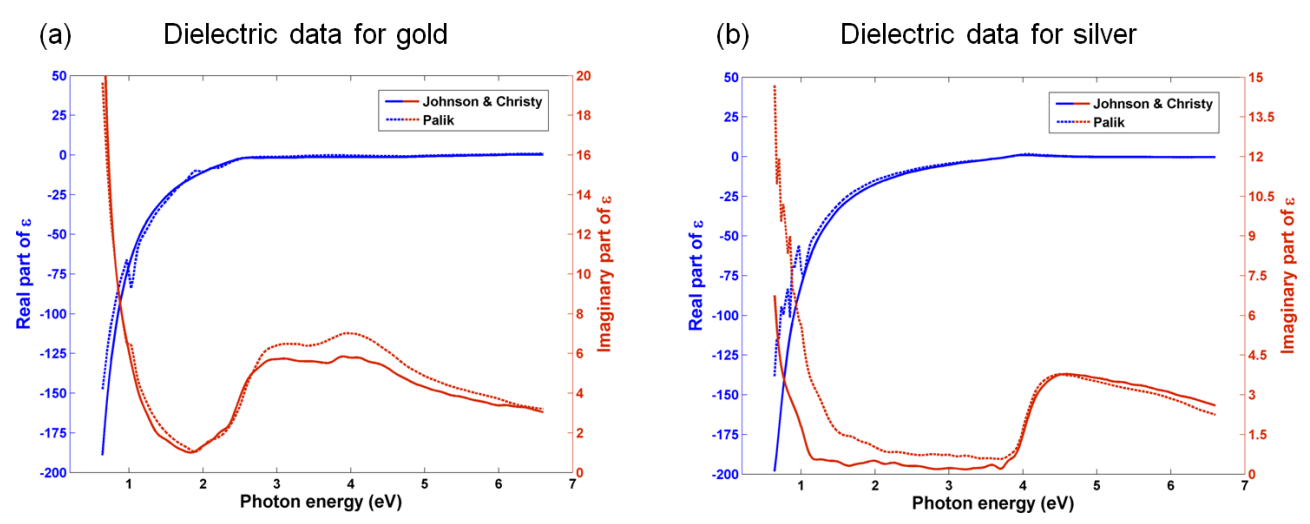

Figure 2.3.: Comparison of the dielectric data for gold (a) and silver (b) obtained from experiment and published in [41] and [43].

By following [4] let us investigate a planar interface between a metal and a dielectric with $\varepsilon_{1}=\varepsilon_{m}$ for the metal at $z<0$, and $\varepsilon_{2}=\varepsilon_{b}$ for the dielectric at $z>0$. The wave equation now has to be solved separately in each region of constant $\varepsilon$ and the corresponding boundary conditions tell us how to match the two solutions at the interface. In general, Maxwell's equations allow two sets of self-consistent solutions with different polarizations - TE (or s-polarized) and TM (or p-polarized) modes. Since we do not get a plasmonic excitation for the latter (see [45] for example), we neglect TM modes and write down the solution of Eq. (3.16) as [4]:

$$
\boldsymbol{E}_{j}=\left(\begin{array}{c}
E_{x_{j}} \\
0 \\
E_{z_{j}}
\end{array}\right) e^{i k_{x} x-i \omega t} e^{i k_{z_{j}} z}, \quad j=1,2
$$

The component $k_{x}$ of the wave vector parallel to the interface is conserved, thus the index $j$ indicating the medium is unnecessary there. The boundary conditions (see App. A.1) yield the following relations

$$
\begin{aligned}
E_{x_{1}}-E_{x_{2}} & =0, \\
\varepsilon_{1} E_{z_{1}}-\varepsilon_{2} E_{z_{2}} & =0,
\end{aligned}
$$

i.e. the parallel field component is continuous, whereas the perpendicular component is discontinuous. To solve (2.4a) the corresponding determinant has to vanish. To- 
gether with Gauss' law [Eq. (3.1a)], we obtain the following relations for the wave vector $\boldsymbol{k}$

$$
k_{x}^{2}+k_{z_{j}}^{2}=\varepsilon_{j} k^{2}=\varepsilon_{j}\left(\frac{\omega}{c}\right)^{2} \longrightarrow \quad k_{z_{j}}=\sqrt{\varepsilon_{j}\left(\frac{\omega}{c}\right)^{2}-k_{x}^{2}}, \quad j=1,2 .
$$

This directly yields the dispersion relation between the wave vector components and the angular frequency $\omega$

\section{Plasmon dispersion relation}

$$
\begin{aligned}
k_{x} & =\frac{\omega}{c}\left(\frac{\varepsilon_{1} \varepsilon_{2}}{\varepsilon_{1}+\varepsilon_{2}}\right)^{1 / 2}, \\
k_{z_{j}} & =\frac{\omega}{c}\left(\frac{\varepsilon_{j}^{2}}{\varepsilon_{1}+\varepsilon_{2}}\right)^{1 / 2}, \quad j=1,2 .
\end{aligned}
$$

We are looking for solutions that are propagating along the surface, i.e. we require a real $k_{x}$.

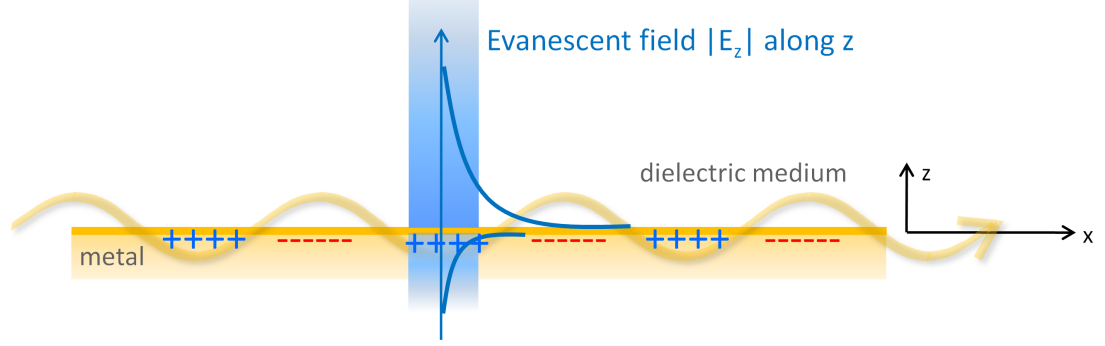

Figure 2.4.: An evanescent wave corresponds to a TE-mode that propagates along the interface of a metal and a dielectric, where the z-component of the electric field decays exponentially.

From the dispersion relation it follows that the conditions for the existence of an 
interface mode are given by

$$
\varepsilon_{1} \varepsilon_{2}<0, \quad \varepsilon_{1}+\varepsilon_{2}<0,
$$

which results in an electromagnetic wave bound to the interface ${ }^{13}$, see Fig. 2.4. The resulting imaginary $k_{z_{j}}$ corresponds to exponentially decaying (so called evanescent) waves.

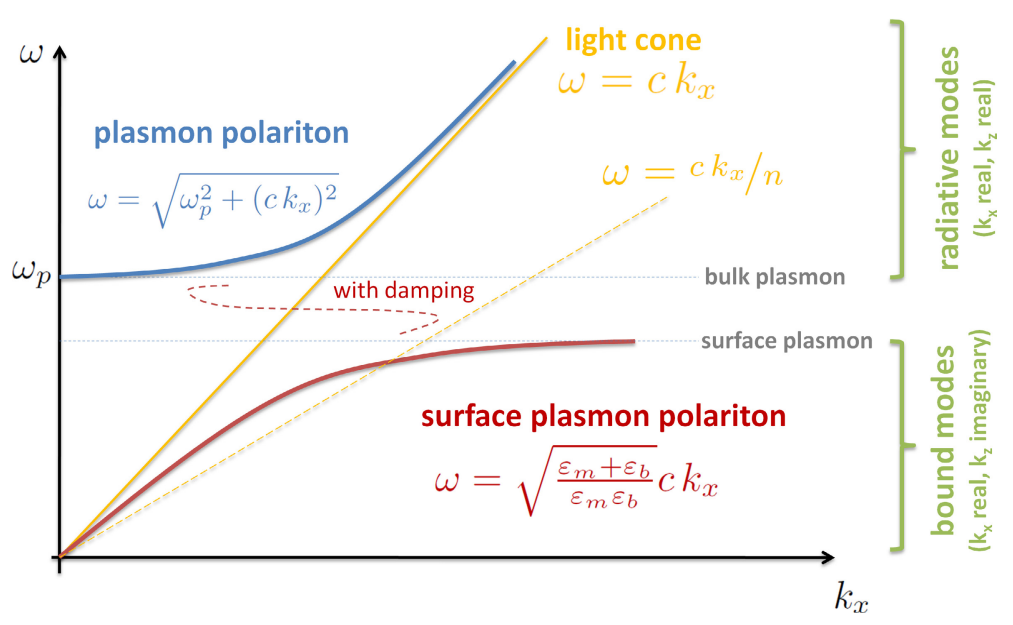

Figure 2.5.: Plasmon dispersion relation for a metal/air interface. Since the dispersion line of plasmons (red line, without damping; blue line for free electrons) does not cross the light cone (yellow line) at any point, it is not possible to excite a surface plasmon at a metal air interface with a light wave. Yet the light cone can be tilted (dotted yellow line) if we change from free space to a dielectric medium.

The dispersion relation (2.6) is plotted in Fig. 2.5. Since the red line of the surface plasmon polariton does not cross the light cone at any point, a direct excitation of surface plasmons with an electromagnetic wave is not possible (the momentum of light is always too small). Nevertheless surface plasmon polaritons can be excited, of course, one just has to provide the missing momentum contribution. This can be done through a tilt of the light line $\omega=c k_{x}$ to $c k_{x} / n$ in a dielectric medium as shown in Fig. 2.5 and Fig. 2.6.

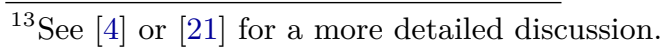


(a)

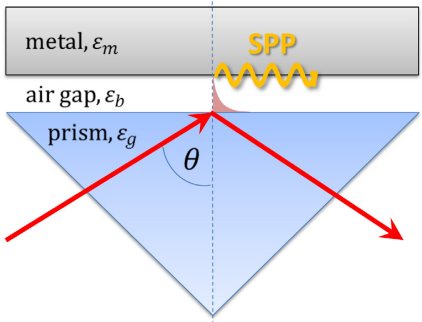

(b) Kretschmann configuration

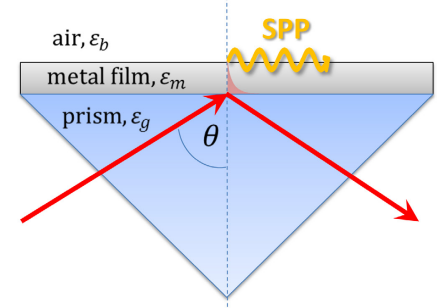

Figure 2.6.: Two configurations that provide the missing momentum contribution discussed in the text for the excitation of surface plasmons. (a) Otto configuration: The total reflection at the prism/air interface generates an evanescent field that excites a SPP at the dielectric/metal interface. This is an useful method when the metal surface should not be damaged, but it is difficult to keep the constant distance (usually of the order of $\lambda$ ) between the metal and the prism. (b) Kretschmann configuration: Here the total reflection at the prism/metal interface generates an evanescent field that excites a surface plasmon at the opposite metal/air interface. The thickness of the metal film must be smaller than the skin depth [see Eq. (2.9)] of the evanescent field.

\section{Skin depth and propagation length}

The imaginary part of the dielectric function is related to the energy dissipation of the material, i.e. if an electromagnetic waves impinges on a metal surface it can only penetrate the solid up to a certain material dependent depth. This so called skin depth $\zeta$ is defined as the distance, where the exponentially decreasing evanescent field $e^{-\left|\boldsymbol{k}_{z_{j}}\right||z|}$ falls to $1 / e[21,44]$ :

\section{Skin depth}

$$
\zeta_{i}=\frac{1}{\left|k_{z i}\right|} \quad \text { or } \quad \begin{cases}\zeta_{b}=\frac{\lambda}{2 \pi}\left(\varepsilon_{b}-\frac{\varepsilon_{m} \varepsilon_{b}}{\varepsilon_{m}+\varepsilon_{b}}\right)^{-1 / 2} & \text { in medium with } \varepsilon_{b}, \\ \zeta_{m}=\frac{\lambda}{2 \pi}\left(\varepsilon_{m}-\frac{\varepsilon_{m} \varepsilon_{b}}{\varepsilon_{m}+\varepsilon_{b}}\right)^{-1 / 2} & \text { in metal with } \varepsilon_{m} .\end{cases}
$$

Since the dielectric background constant $\varepsilon_{b}$ is usually much smaller than the real part 
of $\varepsilon_{m}$, inside the metal Eq. (2.9) can be replaced with the approximation $\zeta \approx \lambda /\left(2 \pi \varepsilon_{m}^{\prime}\right)$, where $\varepsilon_{m}=\varepsilon_{m}^{\prime}+i \varepsilon_{m}^{\prime \prime}$.

If a surface plasmon propagates along a smooth surface, its intensity decreases as $e^{-2 k_{x}^{\prime \prime} x}[21]$, where $k_{x}=k_{x}^{\prime}+i k_{x}^{\prime \prime}$. The length $\delta=1 / 2 k_{x}^{\prime \prime}$ after which the intensity has fallen to $1 / e$ can be defined as the propagation length.

\subsubsection{Particle plasmons}

In general, we have seen that plasmons arise from an interplay of electron density oscillations and the exciting electromagnetic fields. In this sense, we should talk about surface plasmon polaritons and also distinguish the propagating (evanescent) modes at the interface of a metal and a dielectric from their localized counterpart at the surface of metallic particles (so called particle plasmon polaritons). If an electromagnetic wave impinges on a metallic nanoparticle (whose spatial dimension is assumed to be much smaller than the wavelength of light), the electron gas gets polarized (polarization charges at the surface) and the arising restoring force again forms a plasmonic oscillation, see Fig. 2.7.

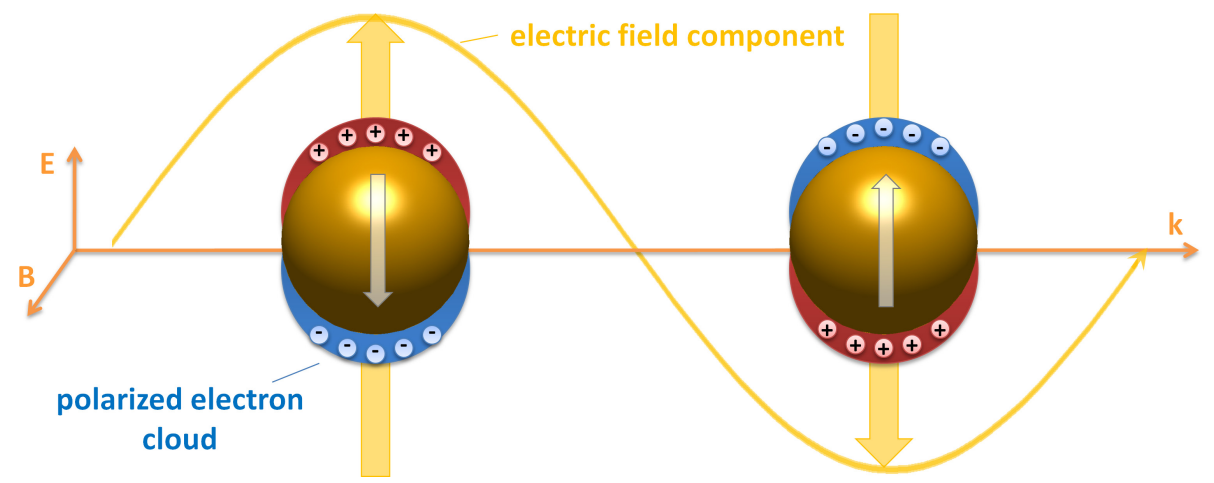

Figure 2.7.: Excitation of particles plasmons through the polarization of metallic nanoparticles. At the resonance frequency the plasmons are oscillating with a $90^{\circ}$ phase difference $\left(180^{\circ}\right.$ above resonance). Also a magnetic polarization occurs, but most of the time it can be neglected for reasons discussed in Sec. 2.5.

The metallic particle thus acts like an oscillator and the corresponding resonance behavior determines the optical properties [46]. Since we solely discuss particle plasmon polaritons in this work, we will furthermore always refer to them. 


\subsection{Tuning the plasmon resonance}

When a metallic nanoparticle is illuminated by white light, the plasmonic resonance determines the color we observe, see Fig. 2.8. This behavior is nothing new: Microscopic gold and silver particles incorporated in the stained glasses of old church windows are responsible for their beautiful lustrous colors ${ }^{14}$. Another very famous example dates back to antiquity - a Roman cup made of dichroic glass illustrating the myth of King Lycurgus ${ }^{15}[49,50]$.

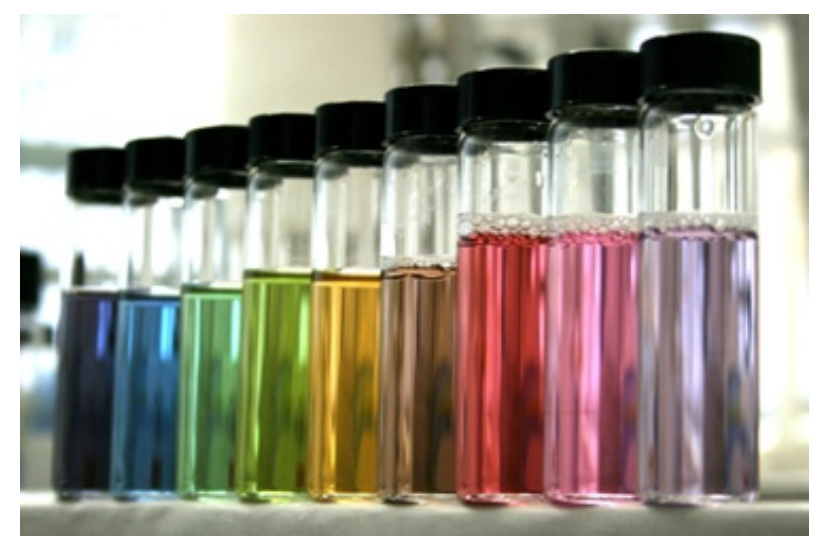

Figure 2.8.: Nanoparticles of various shape and size in solution - the plasmonic resonance determines the color.

(Photo by Carsten Sönnichsen, http://www.nano-bio-tech.de/).

Let us discuss this topic more precisely: We can tune the resonance of the surface plasmon polariton by changing the size or shape of a metallic nanoparticle, as plotted in Fig. 2.9. We recognize that the effect of squeezing a sphere to a rod-like particle has a much bigger impact than increasing its diameter and the upper panel in Fig. 2.9 (b) also shows that the resonance intensity has a maximum for the aspect ratio somewhere between 0.3 and 0.4 .

The plasmonic resonance is not only sensitive to the shape and size of a nanostructure,

\footnotetext{
${ }^{14}$ The windows of Sainte Chapelle in Paris are a very nice example for this: Light transmission through the metal ions in the stained glass strongly depends on the incident and viewing angles. At sunset, the grazing-angle scattering of light by gold particles in the window creates a pronounced red glow that appears to slowly move downward, while intensities of blue tints from ions of copper or cobalt remain the same $[47,48]$.

${ }^{15}$ Lycurgus cup, 4th century AD, British Museum, London.
} 
(a)
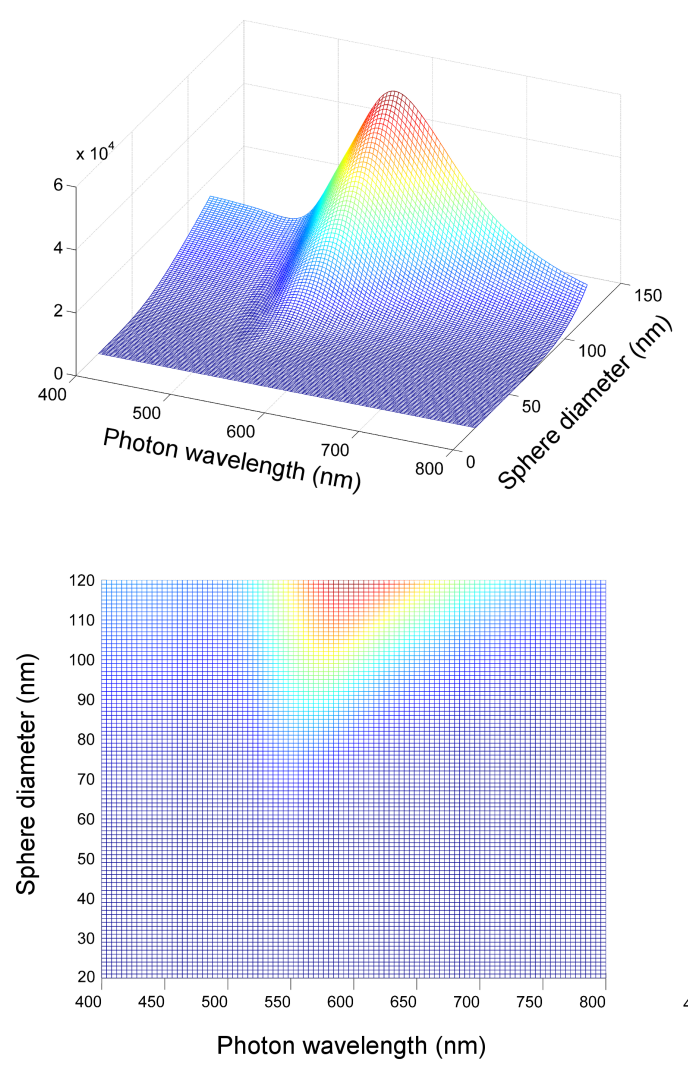

(b) Change of aspect ratio
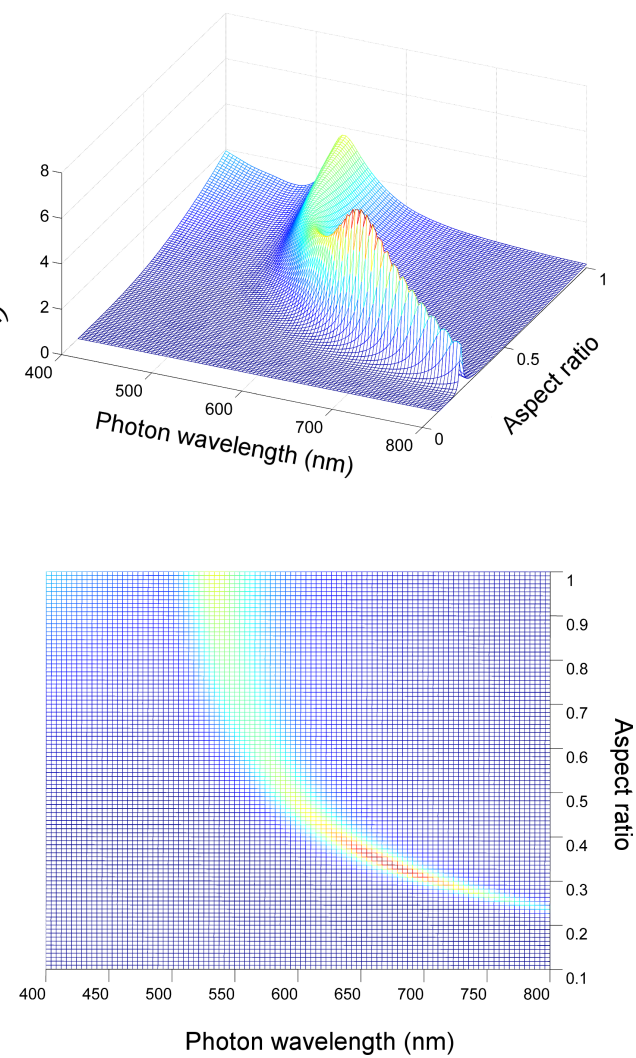

Figure 2.9.: Tuning the resonance of a surface plasmon polariton by changing the diameter of a gold nanosphere (a) or by squeezing its aspect ratio (b). The upper panels show the scattering cross section, in the lower panels we report the corresponding density plots.

also the dielectric medium surrounding the particle plays a key role, see [51] for example. In Fig. 2.10 the sensitivity of a nanorod to the dielectric background $\varepsilon_{b}$ is shown.

Even the slightest change in the dielectric surrounding leads to a detectable shift of the resonance energy. That is the reason why metallic nanoparticles are very suitable for sensing applications: Placing a molecule in the vicinity of a nanoparticle effects the dielectric environment and therefore shifts the plasmon peak. As already mentioned in the introduction, bio-sensing is one of the major applications for plasmonic nanoparticles. Thus naturally the question of the optimal shape and size of a sensor 


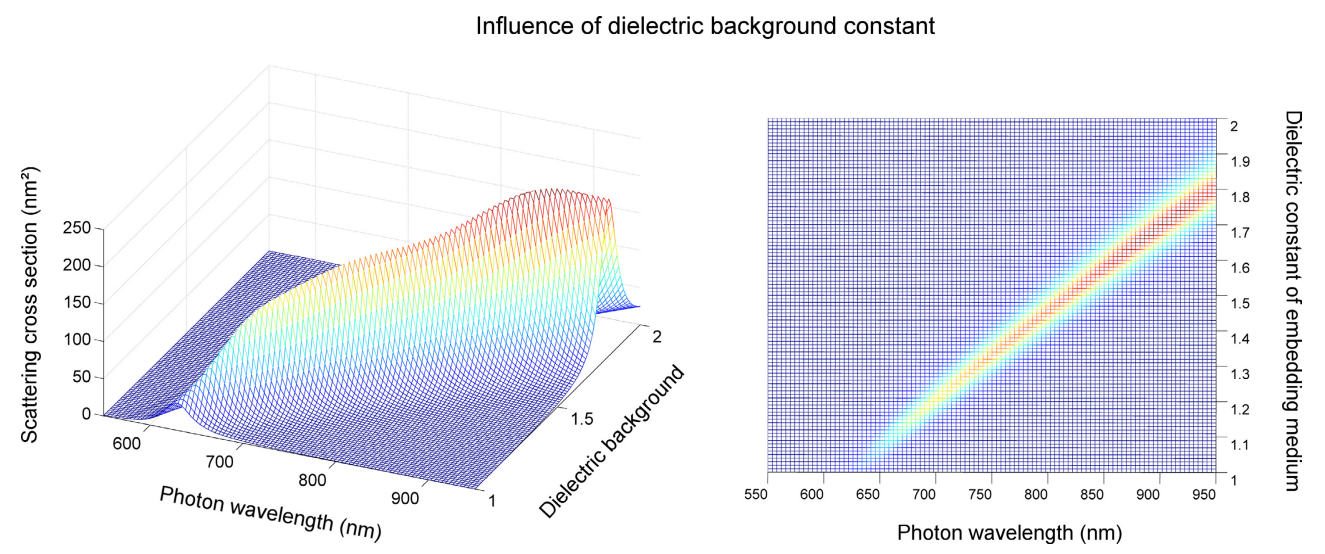

Figure 2.10.: Scattering cross section of a gold nanorod with diameter $10 \mathrm{~nm}$ and an arm length of $35 \mathrm{~nm}$. The dielectric constant $\varepsilon$ of the embedding medium varies from 1.0 to 2.0 , the panel on the right again shows the corresponding density plot.

made of nanoparticles arises. A comprehensive analysis of this question can be found in Chap. 8 and Chap. 9, and we will also briefly highlight the principle of plasmonic sensing in the following subsection.

The influence of the embedding medium on a metallic nanoparticle is a tricky topic as discussed in Sec. 4.5.3. For example, if particles in aqueous solution are investigated, a constant and homogeneous water temperature must be assured because the refractive index of water is temperature dependent [52]. Indeed, the change from $20{ }^{\circ} \mathrm{C}$ to $40{ }^{\circ} \mathrm{C}$ leads to a resonance shift of about $1 \mathrm{~nm}$ [53], which for very accurate sensing applications may become important - especially when heat is generated through an exciting laser field [54].

\subsubsection{Principle of plasmonic (bio-)sensing}

One possible route to plasmonic molecular sensors is given by exploiting the enhancement of the decay rates of fluorophores in the vicinity of MNPs $[55,56]$. The molecule uses the nanoparticle as an antenna in order to emit its energy much faster - the enhancement can be two orders of magnitude and more. In Fig. 2.11 an example published in [55] is shown, where fluorophores were deposited onto two different samples 
of nanodisk-arrays. The molecules absorb in the ultraviolet and emit in the visible regime, which allows a separation of the excitation and emission channel.

(a)

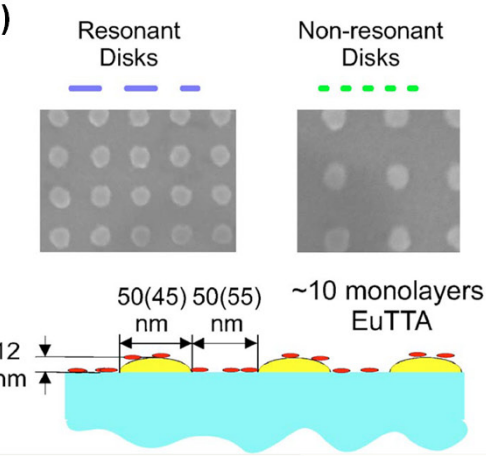

(b)

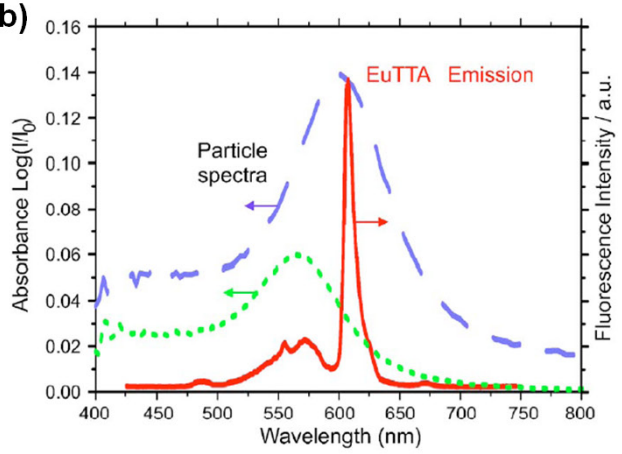

Figure 2.11.: Figure taken from [55]. (a) Electron-microscopic image of two different quadratic arrays of gold nanodisk samples on a silicon dioxide substrate, one geometry is chosen resonant and the other off-resonant to the fluorophore emission. The size parameters and mutual distances are shown in the lower panel. The large interparticle distance of $\sim 50 \mathrm{~nm}$ ensures that coupling effects between the disks can be neglected. (b) Absorbance spectra (dashed and dotted line) of the two nanodisk samples as well as the emission spectrum (solid line, maximum at 612 $\mathrm{nm}$ ) of the fluorophores.

If the plasmon frequency of the disk-arrays is in resonance with the molecular emission (blue dashed line in Fig. 2.11), each disk acts as a supplemental antenna for the molecules. The nonradiative near-field of the fluorophores gets converted into radiating far-field, which leads to the dramatic increase of the radiative decay rate [55]. as shown in Fig. 2.12.

The decay rate of the molecule can be calculated by Fermi's golden rule, as has been shown in [56]. In the cited work the decay rate of the coupled MNP-fluorophore system is derived, which is fully determined by the dyadic Green function of classical electrodynamics. It can be described in terms of a self-interaction, where the molecular dipole polarizes the MNP, and the total electric field acts, in turn, back on the dipole [see Fig. 2.13(b)].

But not only the radiative decay rate gets enhanced, also the nonradiative decay channel is strongly increased because of Ohmic power loss inside the nanodisks as indicated in Fig. 2.13 and discussed in [55]. 

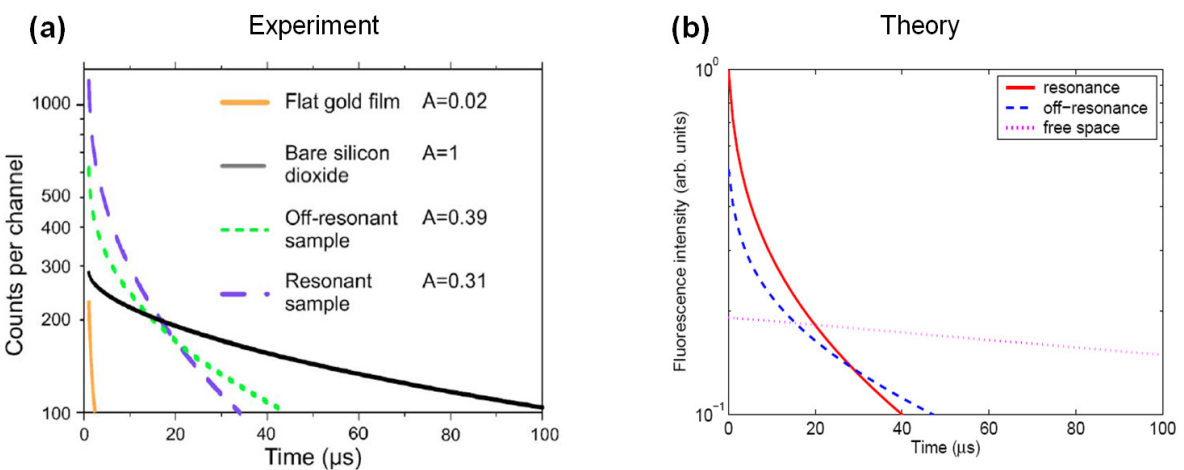

Figure 2.12.: Figure taken from [55]. Panel (a) shows the fitted fluorescence curves for the two samples of Fig. 2.11 (dashed and dotted lines), for a flat gold film (yellow line in lower left corner), and for the bare silicon substrate (black solid line). (b) Fluorescence intensity results of simulations with the boundary element method (see 4.3) for the corresponding setup, for further details see [55].

(a) free molecule

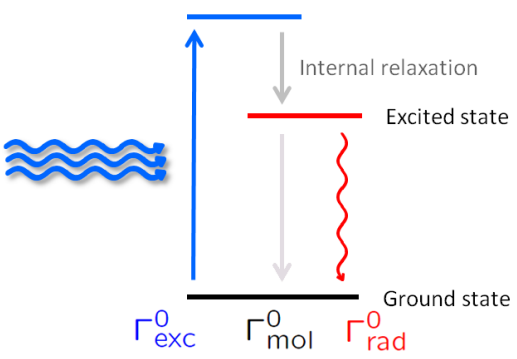

(b) molecule \& nanopartilce

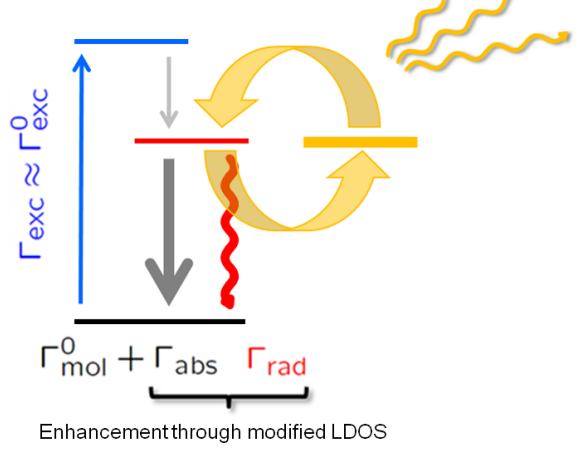

Figure 2.13.: Energy levels, radiative $\left(\Gamma_{\text {rad }}\right)$ and non-radiative $\left(\Gamma_{\text {mol }}\right)$ decay rates of a free molecule (a) and a molecule coupled to a metallic nanoparticle (b). The decay rates for the free fluorophore are indicated with a 0 in the superscript and the excitation is noted as $\Gamma_{\text {ext }}$. The plasmon resonance tuned to the molecular emission enhances both, the radiative as well as the nonradiative decay channel [55].

This example illustrated the cumulative effect of many molecules spread over an array of nanoparticles, but the crux of the whole sensing problem is given by the possibility to detect one single molecule. Here once again the near-field enhancement of metallic nanoparticles is of great importance. In Fig. 2.14 the near-field enhancement of a bowtie nanoantenna is plotted on a logarithmic scale. 

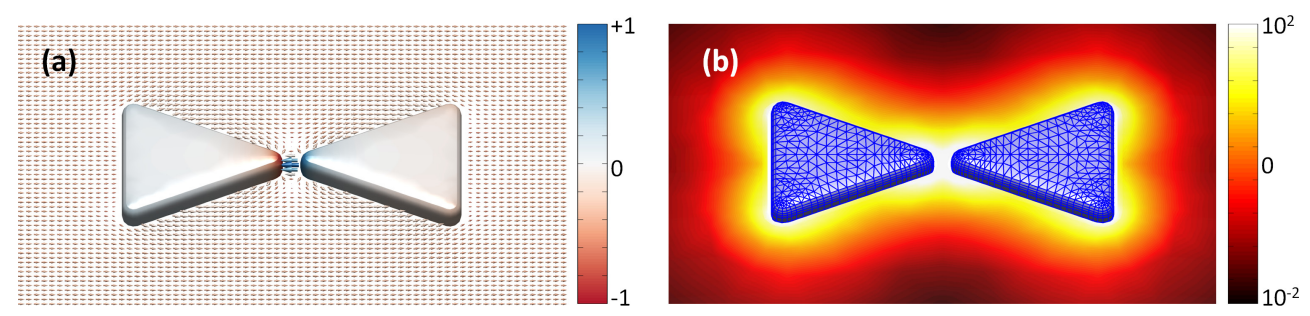

Figure 2.14.: (a) Real part of electric field (in the gap region the field is about 7 times larger than the background) and normalized surface charge, each one at the resonance energy for the gold bowtie nanoantenna with $5 \mathrm{~nm}$ gap distance. (b) Near-field enhancement $|\boldsymbol{E}|^{2} /\left|\boldsymbol{E}_{0}\right|^{2}$ calculated for the same nanoentenna. The triangle size is $45 \mathrm{~nm}$ in $\mathrm{x}, 40 \mathrm{~nm}$ in $\mathrm{y}$ and $8 \mathrm{~nm}$ in z direction.

For a gap distance of $5 \mathrm{~nm}$ we get an strong intensity enhancement as well as a localization in the gap region. Placing a single molecule in the hot spot at the gap leads the way to single molecule sensors, see Fig. 7.4. In [57], for example, single molecule fluorescence enhancements up to a factor of 1340 for gold bowtie nanoantennas have been reported. 


\section{Additional remark}

A nice review about advances in the field of optical-biosensors can be found in [58]. A typical example of the working principle of a sensor based on surface plasmons is shown in Fig. 2.15 below, where the binding of analytes can be measured non-invasively in real time.

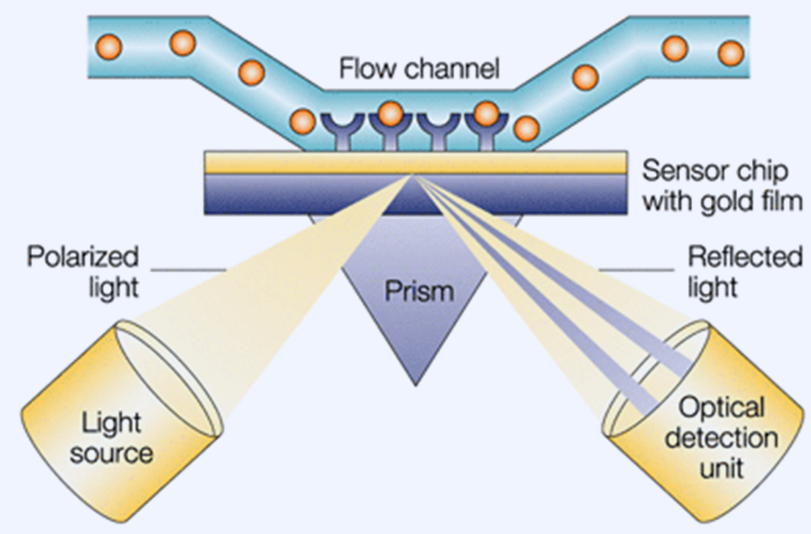

Figure 2.15.: The changes in the refractive index in the immediate vicinity of a surface layer are detected with a sensor chip. The plasmonic resonance is observed as a sharp shadow in the reflected light at an angle that depends on the mass of material at the surface - this angle shifts if biomolecules bind to the surface. (Figure and caption adopted from [58].) 


\subsubsection{Light absorption in solar cells}

Another of the many possible applications that could be revolutionized (or at least very much improved) by plasmonic structures are photovoltaic devices [20,59]. The combination of MNPs and semiconductor materials, for example, allows a considerable reduction in the physical thickness of absorber layers, and yields new possibilities for the design of solar-cells. We don't want to go into the details of this broad research field, but since some of the solar cell aspects are again nice examples for the tunability of the plasmonic resonance we will shortly dwell upon this subject. A review about recent advances at the intersection of plasmonics and photovoltaics can be found in [59] and a brief overview of three different light-trapping thin-film geometries is depicted in Fig. 2.16.
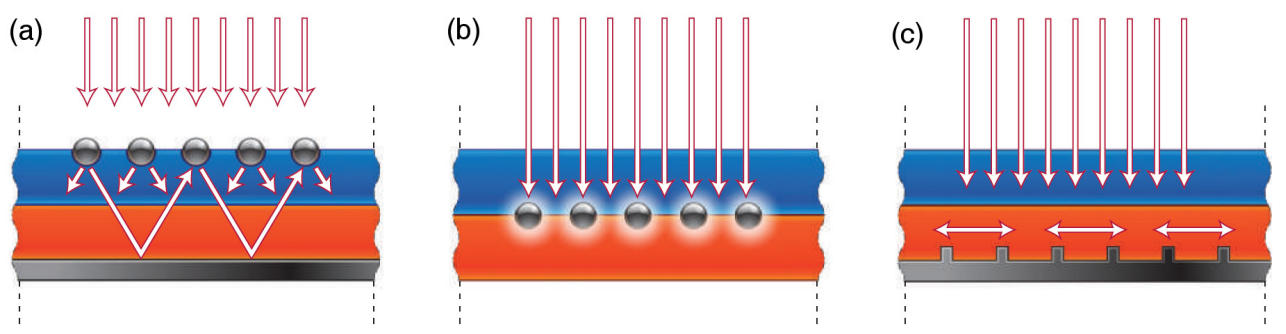

Figure 2.16.: Three different plasmonic geometries for light-trapping for thin-film solar cells, figure and caption taken from [59]. (a) Metallic nanoparticles embedded at the solar cell surface scatter light preferentially into the semiconductor thin film which leads to an increase of the optical path length in the cell. (b) If the particles are embedded in the semiconductor, the creation of electron-hole pairs is caused by the particle's near-field. (c) Light coupling through a corrugated metal back surface.

Unfortunately for the developing of photovoltaic cells our sun is an incoherent black body source and has nothing to do with the compliant light of a laser. An efficient solar device must therefore have a very broad absorption spectrum for all parts of sun light. There are two easy ways to broaden the absorption of a MNP, either by adding an additional coating around the structure or simply by coupling it to another particle, see Fig. 2.17. 


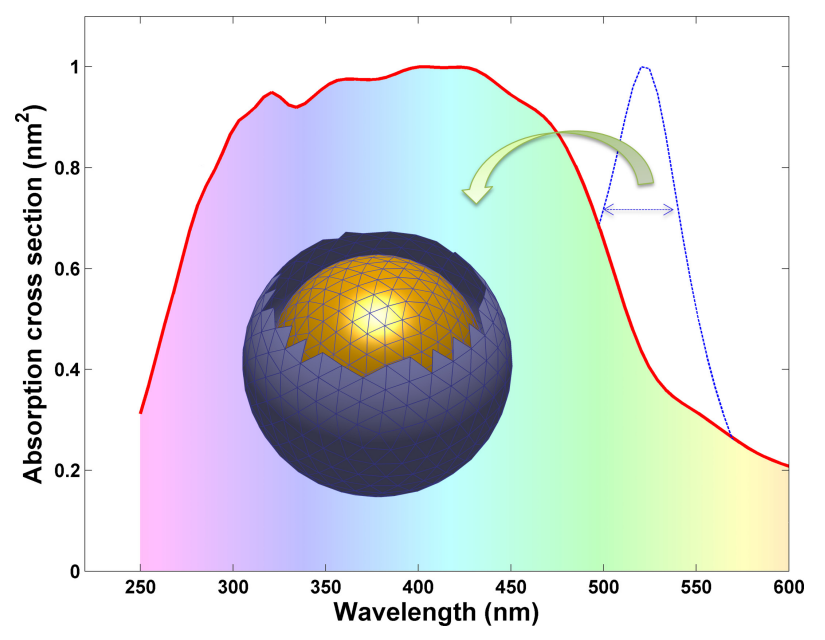

Figure 2.17: The blue dotted line shows the normalized absorption spectrum for a 10 nm gold sphere. Adding an additional layer around the sphere (see inset, $n_{\text {layer }}=$ 10), yields the broadened red absorption line (again normalized to 1). The light color range was approximated according to Fig. A.2.

\section{Additional remark}

The coupling of two or more metallic nanoparticles leads to a hybridization of the energy levels. Fig. 2.18 shows the example for spherical dimers.

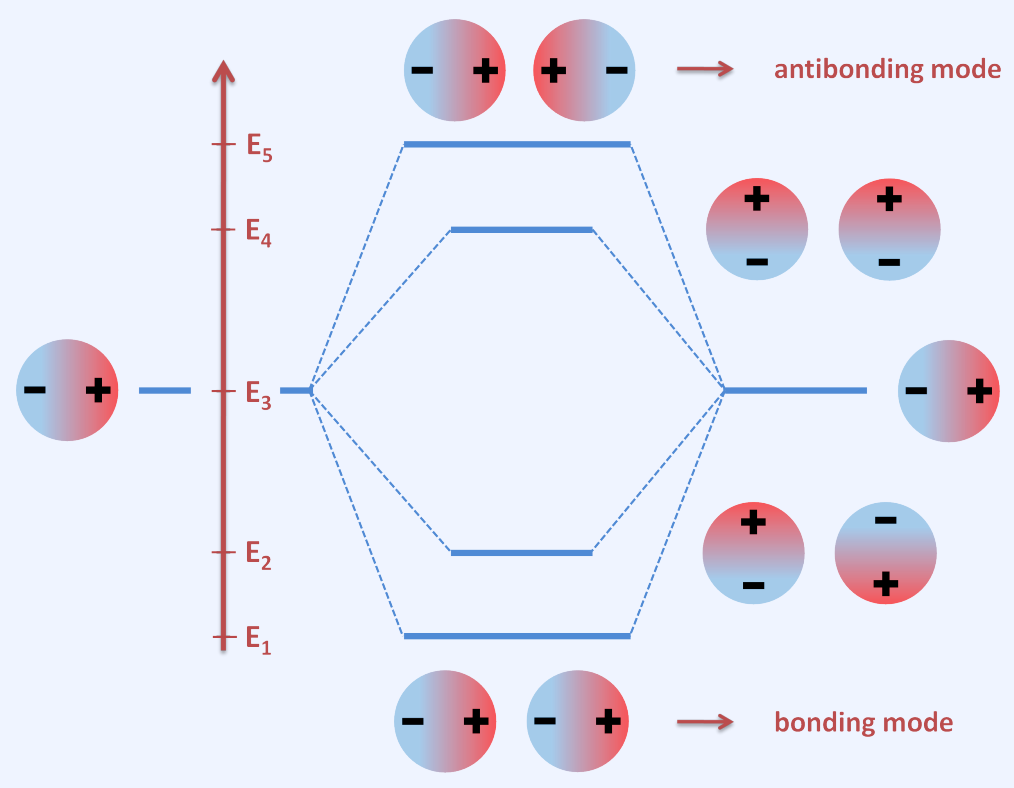

Figure 2.18.: Energy levels of two coupled spherical nanoparticles (see [60,61]), note the occurrence of bonding and antibonding modes. 


\subsection{Damping mechanisms of surface plasmon polaritons}

As discussed at the beginning of this chapter, a plasmon is formed when a coherent charge density oscillation is induced in the electron gas of a metallic nanoparticle by an external excitation. This collective motion of the electrons can easily be disturbed, for example by scattering events that destroy the phase coherence. One can imagine this dephasing by simply kicking an electron out of the lock-step march, due to scattering with impurities, phonons, other electrons, and so on. The electron still has its kinetic energy but the phase coherence gets destroyed. Fig. 2.19 gives an overview about the different decay channels and in Fig. 2.20 the corresponding time scales are plotted.

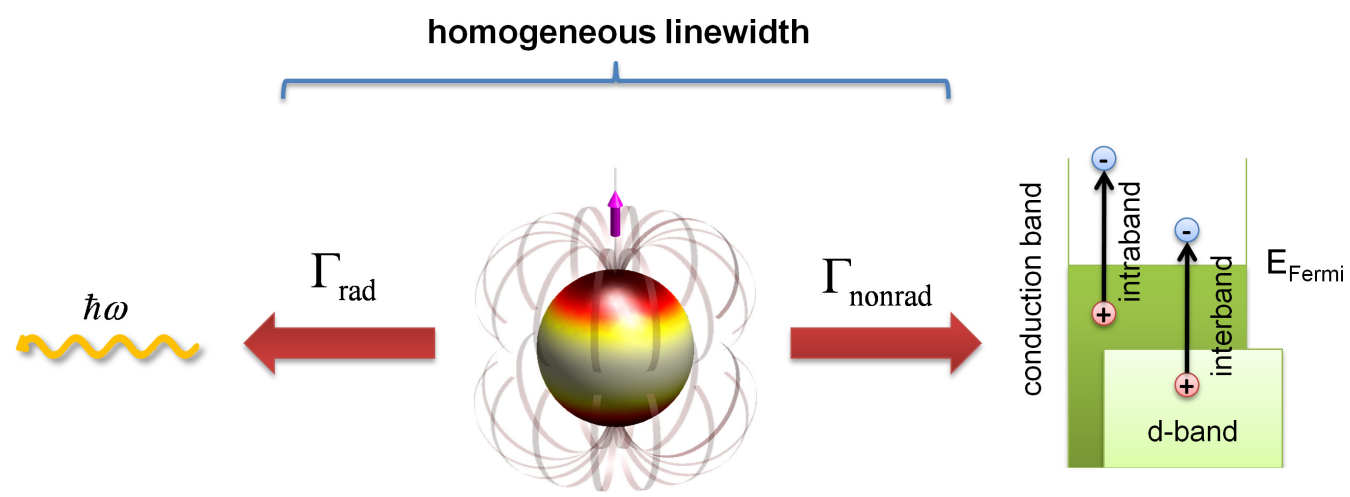

Figure 2.19.: Usually a particle plasmon shares the destiny of a mayfly, albeit on a different time scale: After a quite short existence it is doomed to decay. It can either decay radiatively (left) and emit photons or lose its energy non radiatively via intra- and interband transitions (right), also see Fig. 2.20. Both decay channels contribute to the homogeneous linewidth $\Gamma$.

The radiative and nonradiative break up and decay processes of plasmons result in highly excited electron-hole pairs, which thermalize by further collision processes on a sub-ps time scale $[62,63]$ to a distribution of "hot" electrons ${ }^{16}$ and holes [46].

The decay time $\tau$ of a particle plasmon oscillation can be determined from the homogeneous linewidth $\Gamma$ of the spectral resonance of a plasmon, see App. A.3. $\Gamma$ is defined as the full width at half maximum (FWHM) and is inversely proportional to

\footnotetext{
${ }^{16}$ Since the heat capacity of the electronic system is much smaller than that of the ion lattice, an excitation by femtosecond laser pulses can generate extremely high electron temperatures [46].
} 


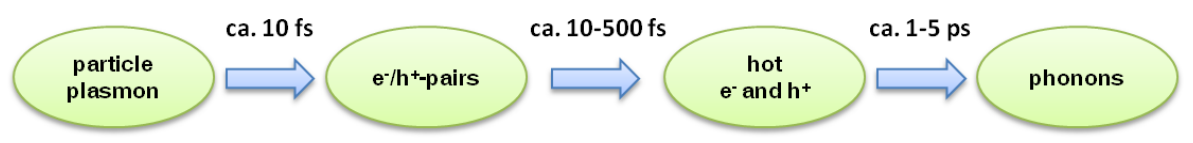

Figure 2.20.: List of typical plasmon decay times obtained from [46]. After about $10 \mathrm{fs}$ the particle plasmon decomposes into electron-hole pairs, which further thermalize on a sub-ps timescale to a distribution of hot electrons and holes. Finally through electron-phonon coupling the energy is transfered to the lattice as heat.

the decay time $\tau: \Gamma \propto \tau^{-1}$, see Eq. (A.17). Fig. 2.21 shows an example for a nanorod antenna, also see Fig. 13.1.

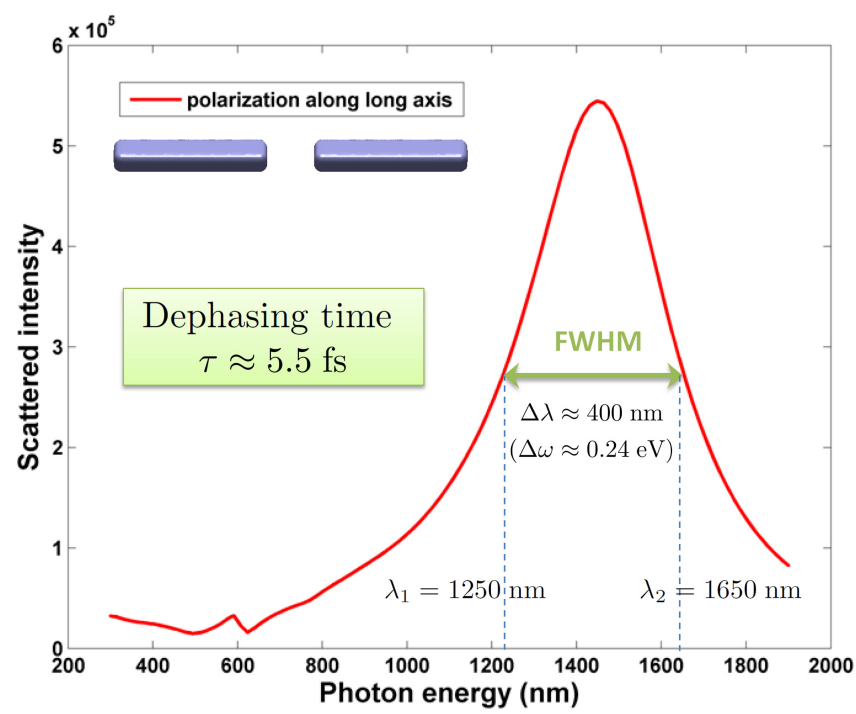

Figure 2.21.: Homogeneous linewidth (FWHM) $\Gamma$ and plasmon decay time $\tau$ for a gold nanorod antenna (rod length $280 \mathrm{~nm}$, width $60 \mathrm{~nm}$, height $40 \mathrm{~nm}$, and gap distance $65 \mathrm{~nm}$ ). The resulting decay time $\tau \approx 5.5$ fs for this geometry has also been verified by autocorrelation measurements, see Chap. 13 .

Increased damping, for example caused by defects in the nanoparticles' crystal structure, thus leads to a broadening of the spectral linewidth. Note that $\Gamma$ can vary more than a factor of ten for different nanoparticle geometries - defining the quality of a plasmonic sensor simply over the shift of the resonance may therefore be a little bit simplistic. In Chap. 8 we will introduce several 'figures of merit' to allow a better quality comparison of different plasmonic sensors. 
As discussed in Chap. 7, for the direct measurement of the temporal evolution of particle plasmons ultrashort laser pulses are necessary. These pulses have become available in the past decade and thus probing ultrafast plasmon dynamics directly in the time domain with fs time-resolution has become possible, see Chap. 13.

\subsection{Magnetic effects}

A light wave always consists of an oscillating electric and magnetic field, the one never occurring without the other. But since at optical frequencies the value of the magnetic permeability $\mu=\left(1+4 \pi \chi_{m}\right) \approx 1$, the magnetic component of light generally plays an insignificant role and can often be neglected. This aspect can be easily understood in a simplified picture with the Lorentz force $\boldsymbol{F}_{L}$ [64-66]. As we have highlighted above, the electron cloud of a metallic nanoparticle can interact with an impinging light wave. In classical electrodynamics the effect of the electromagnetic field on a moving charge $q$ is described through [66]

\section{Lorentz force in Gaussian units}

$$
\boldsymbol{F}_{L}=q\left[\boldsymbol{E}+\left(\frac{\boldsymbol{v}}{c} \times \boldsymbol{B}\right)\right] .
$$

The ratio of the velocity $|\boldsymbol{v}|$ to the speed of light $c$ determines the ratio of the magnetic contribution to it's electric counterpart [65]. The norm of the charge velocity in solid state physics is roughly given by the Fermi velocity ${ }^{17} v_{F}$ which implies for the ratio that $v_{F} / c \approx 1 / 300$ [65]. The magnetic response of a material is determined by the magnetic susceptibility $\chi_{m}$, which scales as $\left(v_{F} / c\right)^{2}$. Thus it follows that the magnetic response is four orders of magnitude weaker than the ease with which the same material is polarized [64].

${ }^{17}$ Numerical value for gold and silver particles $v_{F} \approx 1.4^{\mathrm{nm}} / \mathrm{fs}$, Fermi energy $E_{F} \approx 5.53 \mathrm{eV}$ respectively $[39])$. 
When we try to detect light in experiments, we are most of the time blind to its magnetic part and can only perceive its electric component [64]. One way to visualize both, the magnetic- and electric-field distribution of propagating light, has been demonstrated by Burresi et al. in [64] through the combination of near-field measurements and metamaterials (see Fig. 2.22).

(a)

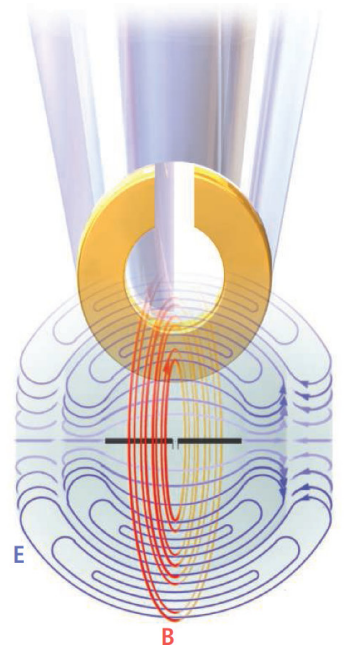

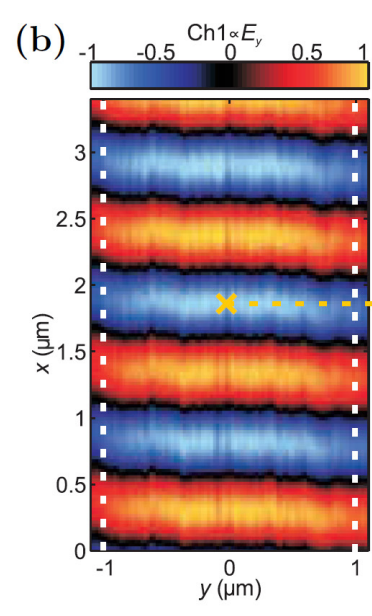

Figure 2.22.: (a) Scheme of the experimental setup used by [64] to measure the electric (blue lines) and magnetic (red lines) field of optical waves, figure taken from [65]. Burresi et al. used a metallic splitring resonator at the tip of a glass fiber to read out the scattered light from a scanning near-field optical microscope (SNOM). (b) Normalized distributions of $E_{x}$ and $\boldsymbol{B} \mathrm{s}_{z}$ published in [64], see discussion therein. The yellow dashed line shows the $\pi / 2$ phase shift. 


\section{Theory}

The unification of the theories describing electric and magnetic aspects of our world was one of the great scientific achievements in the 19th century [67] and brought us a very successful part of theoretical physics: classical field theory. A detailed overview about the historical evolution from René Descartes up to Maxwell and Lorentz can be found in the excellent book of Whittaker [68]. After the revolution of our understanding of the basic forces and constituents of matter in the last 100 years, classical electrodynamics found its place in a sector of the unified description of particles and interactions known as the standard model [66].

\subsection{Quantum versus classical field theory}

Atoms and their corresponding electromagnetic fields fluctuate quite rapidly on the nanoscale, so usually we need to average over a larger region to obtain a macroscopic theory. In this sense the concept of the ordinary electromagnetic fields is a classical notion. It can be thought of as the classical limit (limit of large photon numbers and small momentum and energy transfers) of quantum electrodynamics (QED) ${ }^{1}$ [66]. But nanoparticles are situated in the gray zone between the micro- and macrocosm - they are very small compared to classical objects but they still consist of several thousands to millions of atoms. Nevertheless surface plasmons are bosonic quasiparticles and have a true quantum nature that has been demonstrated by tunneling experiments for example, see [70]. Hence, for the theoretical description we can either come from the bottom and try to apply a quantum mechanical treatment or we can deal with plasmonic structures in terms of classical field theory (and hope that the particles are not too small).

\footnotetext{
${ }^{1} \mathrm{~A}$ nice introduction into the topic of QED can be found in [69] for example.
} 
At which point is it justified, that we neglect (or at least gloss over) the discrete photon aspect of the electromagnetic field and change from QED to Maxwell's theory? In the domain of macroscopic phenomena virtually always, as the examples discussed in [66] elucidate: The root mean square electric field one meter away from a $100 \mathrm{watt}$ light bulb is of the order of $50 \mathrm{~V} / \mathrm{m}$ and there are of the order of $10^{15}$ visible photons per $\mathrm{cm}^{2}$ per second. Similarly, an antenna that emits isotropically with a power of 100 watts at $10^{8} \mathrm{~Hz}$ produces a root mean square electric field of only $0.5 \mathrm{mV} / \mathrm{m}$ at a distance of 100 kilometers, but this still corresponds to a flux of $10^{12}$ photons per $\mathrm{cm}^{2}$ per second. Ordinarily an apparatus will not be sensitive to the individual photons; the cumulative effect of many photons emitted or absorbed will appear as a continuous, macroscopically observable response. Then a completely classical description in terms of the Maxwell equations is permitted and is appropriate.

A rough estimate for the justification of a classical treatment is given by a high number of involved photons where at the same time their momentum has to be small compared to the material system ${ }^{2}$. This is true for metallic nanoparticles and in linear response, one can employ the fluctuation-dissipation theorem to relate the dielectric response to the dyadic Green tensor of Maxwell's theory where all the details of the metal dynamics are embodied in the dielectric function. This is exactly what we are going to do, we will hide the quantum-mechanical properties of matter in their dielectric description which is obtained by experiment (see Fig. 2.2 and [41]). In this way, we are communicating with the microscopic world via $\varepsilon$ and $\mu$.

Nevertheless, the whole concept of a dielectric function becomes questionable if the investigated nanoparticles are too small (caution for structures below $5 \mathrm{~nm}$ may be justified). Also if coupled particles get very close to each other, the onset of screening effects and electron tunneling across the gap region significantly modifies the optical response as reported in [72]. In the cited work, the authors present a fully quantum mechanical description of nanoparticle dimers in terms of time-dependent density functional theory and state that quantum effects for dimers become important for dimer separations below $1 \mathrm{~nm}$.

\footnotetext{
${ }^{2}$ Because of energy and momentum conservation at least the time averaged electromagnetic field can still be treated in a classical way, also for clearly quantum mechanical processes like spontaneous emission [66]. A nice discussion of this topic is also given in the introductory chapters of [71]
} 


\subsection{Maxwell's theory of electromagnetism}

We treat the electromagnetic fields as three-dimensional vector fields. Such fields are fully determined by their divergence and curl - that explains the structure and appearance of their mathematical description: Maxwell's equations. In Gaussian units and in their macroscopic version they read as [66]

$$
\begin{array}{rlrl}
\text { Macroscopic Maxwell equations in atomic units } & \\
\nabla \cdot \boldsymbol{D}(\boldsymbol{r}, t) & =4 \pi \varrho(\boldsymbol{r}, t), & & \text { (Gauss's Law) } \\
\boldsymbol{\nabla} \cdot \boldsymbol{B}(\boldsymbol{r}, t) & =0, & & \text { (magnetic analogon) } \\
\boldsymbol{\nabla} \times \boldsymbol{H}(\boldsymbol{r}, t) & =\frac{4 \pi}{c} \boldsymbol{j}+\frac{1}{c} \frac{\partial \boldsymbol{D}(\boldsymbol{r}, t)}{\partial t}, & & \text { (Ampère's Circuital Law) } \\
\boldsymbol{\nabla} \times \boldsymbol{E}(\boldsymbol{r}, t) & =-\frac{1}{c} \frac{\partial \boldsymbol{B}(\boldsymbol{r}, t)}{\partial t} . & & \text { (Faraday's Induction Law) }
\end{array}
$$

Here $\boldsymbol{B}=\mu \boldsymbol{H}$ is the magnetic field (magnetic permeability $\mu=1$ throughout, see Sec. 2.5), $\boldsymbol{D}=\varepsilon \boldsymbol{E}$ is the dielectric displacement ${ }^{3}, \varrho$ the free charge density, $c$ the speed of light, and $j$ the current density.

Maxwell's equations are partial differential equations of first order. In many cases they are linear ${ }^{4}$ in the fields $\boldsymbol{E}$ and $\boldsymbol{B}$. Because of this linearity it is sufficient to only investigate time harmonic fields, any complex solution of the system can then be described as a superposition of them. Henceforth we will use

$$
\boldsymbol{E}(\boldsymbol{r}, t)=\boldsymbol{E}(\boldsymbol{r}) e^{-i \omega t}, \quad \boldsymbol{B}(\boldsymbol{r}, t)=\boldsymbol{B}(\boldsymbol{r}) e^{-i \omega t}
$$

\footnotetext{
${ }^{3}$ This relation connects the microscopic response with a macroscopic field, a more detailed discussion follows in Sec. 3.2.1.

${ }^{4}$ Nonlinear effects may arise at interaction with fiber glass or certain magnetic materials and many other systems.
} 


\section{Additional remark}

The inverse square law of the electrostatic force was shown quantitatively in experiments by Coulomb ${ }^{5}$ and Cavendish ${ }^{6}$ [66]. Applying the divergence theorem together with Gauss's law allows the derivation of the first of Maxwell's equations, Eq. (3.1a). But Coulomb's inverse square law also leads to another remarkably condition: The photon has to be a massless particle [66]! We can verify this hypothesis solely with experiments, and Maxwell's equations are based on this assumption. The consequences of a massive photon are once again discussed in [66], for example, and the experimental verification of Coulomb's law already gives a very good upper limit for the photon mass $m_{\gamma}$, see [73]. Very accurate results for $m_{\gamma}$ can be obtained by measuring the magnetic field of earth, viz.

$$
m_{\gamma}<4 \times 10^{-51} \mathrm{~kg}
$$

or the cosmic magnetic vector potential, see [74]. Also the Schumann resonances (stationary electromagnetic waves along the circumference of the earth) allow for a very simple but surprisingly accurate estimation of the upper limit of $m_{\gamma}$.

If no sources are present $(\varrho=0, j=0)$ Maxwell's equations reduce to

\section{Maxwell's equations in vacuum}

$$
\begin{array}{ll}
\boldsymbol{\nabla} \cdot \boldsymbol{E}=0, & \boldsymbol{\nabla} \times \boldsymbol{B}-\frac{1}{c} \frac{\partial \boldsymbol{E}}{\partial t}=0, \\
\boldsymbol{\nabla} \cdot \boldsymbol{B}=0, & \boldsymbol{\nabla} \times \boldsymbol{E}+\frac{1}{c} \frac{\partial \boldsymbol{B}}{\partial t}=0 .
\end{array}
$$

\footnotetext{
${ }^{5}$ Born $14^{\text {th }}$ June 1736 in Angoulême; $\dagger 23^{\text {th }}$ August 1806 in Paris.

${ }^{6}$ Born $10^{\text {th }}$ October 1731 in Nice; $\dagger 24^{\text {th }}$ February 1810 in London.
} 


\subsubsection{Linear and nonlinear optical response}

If we apply an external electric field to a polarizable (dielectric) medium, the electrons in the material response with a microscopic shift but still remain bound to their associated atoms. The cumulative effect of all displaced electrons results in a macroscopic polarization of the material that can be described by a net charge distribution. In terms of Maxwell's theory it becomes useful if we distinguish between this bound charge distribution and that of free charges, which we have introduced as $\varrho$ in Eq. (3.1a). The contribution of the bound charges is incorporated elsewhere, but let us discuss this in more detail. The electromagnetic fields obey the following relations

$$
\boldsymbol{D}=\boldsymbol{E}+4 \pi \boldsymbol{P}, \quad \boldsymbol{B}=\boldsymbol{H}+4 \pi \boldsymbol{M},
$$

where $\boldsymbol{P}$ is the dipole moment per unit volume and $\boldsymbol{M}$ refers to the magnetic moment per unit volume. Since we focus our attention only to nonmagnetic media, we can set $\boldsymbol{M} \equiv 0$ (see Sec. 2.5) and combine Maxwell's equations to

$$
\nabla^{2} \boldsymbol{E}-\nabla(\nabla \cdot \boldsymbol{E})-\frac{1}{c^{2}} \frac{\partial^{2} \boldsymbol{E}}{\partial t^{2}}-\frac{4 \pi \partial^{2} \boldsymbol{P}}{c^{2} \partial t^{2}}=0
$$

In principle, one now requires a full microscopic theory of the response of a particular material to relate the macroscopic electric field $\boldsymbol{E}$ to the polarization $\boldsymbol{P}[66,75]$. Making some assumptions about the relationship between $\boldsymbol{P}$ and $\boldsymbol{E}$ will make our lives much more easy.

The change in electrostatic potential over distances of the order of an angstrom can be several electron volts. In this sense, an electron bound to an atom or molecule, or moving through a solid or dense liquid, experiences electric fields of the order of $10^{9} \mathrm{~V} / \mathrm{cm}[75]$. The laboratory fields of interest are then small compared to the electric fields experienced by the electrons in the atoms and molecules of the investigated matter. In this circumstance, we can expand $\boldsymbol{P}(\boldsymbol{r}, t)$ in a Taylor series in powers of the macroscopic field ${ }^{7} \boldsymbol{E}(\boldsymbol{r}, t)$. The $\alpha$ th Cartesian component of the dipole moment per unit volume is a function of the three Cartesian components of the electric field $E_{\beta}=$

\footnotetext{
${ }^{7}$ Another method sometimes discussed in literature models the atomic or molecular structure explicitly. There one relates the dipole moment per unit volume to that of an atomic or molecular constituent and writes this as a Taylor series similar to our approach, see $[75,76]$ and references therein.
} 
$E_{\beta}(\boldsymbol{r}, t)$, with $\beta \in\{x, y, z\}$; therefore we can write the Taylor series as follows:

$$
\begin{aligned}
P_{\alpha}(\mathbf{r}, t)=P_{\alpha}^{(0)}+\sum_{\beta}\left(\frac{\partial P_{\alpha}}{\partial E_{\beta}}\right)_{0} E_{\beta}+\frac{1}{2 !} & \sum_{\beta \gamma}\left(\frac{\partial^{2} P_{\alpha}}{\partial E_{\beta} \partial E_{\gamma}}\right)_{0} E_{\beta} E_{\gamma}+ \\
& +\frac{1}{3 !} \sum_{\beta \gamma \delta}\left(\frac{\partial^{3} P_{\alpha}}{\partial E_{\beta} \partial E_{\gamma} \partial E_{\delta}}\right)_{0} E_{\beta} E_{\gamma} E_{\delta}+\cdots
\end{aligned}
$$

Here we have assumed that the dipole moment $\boldsymbol{P}(\boldsymbol{r}, t)$ depends on the electric field $\boldsymbol{E}$ at the same point $\boldsymbol{r}$ in space and the same time $t$, which is not really a realistic assumption - we will introduce a more proper treatment in the next section and we will see, that we can incorporate certain nonlocal aspects in the susceptibility tensor.

In this work, we are interested in dielectric materials within which any dipole moment is induced by the external field. Therefore the electric dipole moment per unit volume at zero field, $P_{\alpha}^{(0)}$, vanishes ${ }^{8}$, and we will henceforth write

$$
P_{\alpha}(\mathbf{r}, t)=\sum_{\beta} \chi_{\alpha \beta}^{(1)} E_{\beta}+\sum_{\beta \gamma} \chi_{\alpha \beta \gamma}^{(2)} E_{\beta} E_{\gamma}+\sum_{\beta \gamma \delta} \chi_{\alpha \beta \gamma \delta}^{(3)} E_{\beta} E_{\gamma} E_{\delta}+\cdots
$$

where the susceptibilities $\chi^{(i)}$ are tensors of $(i+1)$ th rank. $\chi^{(1)}$ is the ordinary susceptibility of dielectric theory (usually a diagonal matrix) and $\chi^{(2)}, \chi^{(3)}$ are referred to as the second and third order susceptibilities, respectively. Now we can decompose the dipole moment into a part which is linear in the electric field, and one part which is nonlinear:

$$
P_{\alpha}(\mathbf{r}, t)=P_{\alpha}^{(L)}(\mathbf{r}, t)+P_{\alpha}^{(N L)}(\mathbf{r}, t)
$$

where

\footnotetext{
${ }^{8}$ The electrical analogs of ferromagnets, which possess a spontaneous magnetization per unit volume, are the so called ferroelectrics. In these materials the dipole moment $\boldsymbol{P}^{(0)}$ in the absence of an electric field is nonzero and leads to the presence of a static, macroscopic electric field, $\boldsymbol{E}^{(0)}(\boldsymbol{r})$. Such time independent effects may be analyzed by the methods of electrostatics and can be accounted for by including an effective charge density $\varrho_{p}=-\boldsymbol{\nabla} \cdot \boldsymbol{P}^{(0)}$, for example.
} 


\section{Linear and nonlinear dipole moment per unit volume}

$$
\begin{aligned}
P_{\alpha}^{(L)}(\mathbf{r}, t) & =\sum_{\beta} \chi_{\alpha \beta}^{(1)} E_{\beta}, \\
P_{\alpha}^{(N L)}(\mathbf{r}, t) & =\sum_{\beta \gamma} \chi_{\alpha \beta \gamma}^{(2)} E_{\beta} E_{\gamma}+\sum_{\beta \gamma \delta} \chi_{\alpha \beta \gamma \delta}^{(3)} E_{\beta} E_{\gamma} E_{\delta}+\cdots
\end{aligned}
$$

With the electric susceptibility $\chi_{e}$ (simplified symbol instead of $\chi^{(1)}$ ) we now obtain the previously discussed relation, where the microscopic response is incorporated in the dielectric function:

$$
\boldsymbol{D}=\boldsymbol{E}+4 \pi \boldsymbol{P}=\left(1+4 \pi \chi_{e}\right) \boldsymbol{E}=\varepsilon \boldsymbol{E}
$$

Furthermore we now have a clear distinction between linear and nonlinear optics: If we insert $P_{\alpha}^{(L)}(\boldsymbol{r}, t)$ into Maxwell's equations we obtain a description of electromagnetic wave propagation in (possibly crystalline) media, described by an electric susceptibility tensor $\chi_{\alpha \beta}$, in linear response. All nonlinear effects are part of the higher order susceptibilities.

Since $\boldsymbol{P}$ and $\boldsymbol{E}$ are vectors, and thus are odd under inversion symmetry, $\chi^{(2)}$ must vanish in any material that is left invariant in form under inversion ${ }^{9}$. But if the symmetry is broken (at the interface from one medium to another, for example) or in the case of surface imperfections [77], we also get $\chi^{(2)}$ contributions for centrosymmetric materials like gold or silver, see Sec. 7 and [78].

\subsubsection{Nonlocal in space and time}

The macroscopic field $\boldsymbol{E}(\boldsymbol{r}, t)$ acts as a driving field that leads to a rearrangement of the electrons and nuclei in the material. The result is the induced dipole moment $\boldsymbol{P}$,

\footnotetext{
${ }^{9}$ This is the case for metals like $\mathrm{Au}$ or $\mathrm{Ag}$, for the semiconductors $\mathrm{Si}$ and Ge as well as for liquids, gases, and for a number of other common crystals. The interested reader may find a very useful compilation of the nonzero elements of $\chi^{(2)}$ and $\chi^{(3)}$ for crystals of various symmetry in [76].
} 
which of course will not be built up instantaneously, but is instead the consequence of the response of the system over some characteristic time interval $t-t^{\prime}>0$ in the recent past. If, on the other hand, we consider an incident electric field well localized in space, it will lead to an electronic rearrangement in a certain small region of the material. Because of the interaction with neighboring constituents, the material gets polarized in the vicinity of the excitation as well. It follows then that the dipole moment $\boldsymbol{P}(\boldsymbol{r}, t)$ depends not only on the field at time $t$ and position $\boldsymbol{r}$, but must be written as a convolution in space and time (exemplified only for linear response)

$$
P_{\alpha}^{(L)}(\mathbf{r}, t)=\sum_{\beta} \int_{\mathbb{R}^{3} \otimes \mathbb{R}} d^{3} r^{\prime} d t^{\prime} \chi_{\alpha \beta}^{(1)}\left(\boldsymbol{r}-\boldsymbol{r}^{\prime}, t-t^{\prime}\right) E_{\beta}\left(\boldsymbol{r}^{\prime}, t^{\prime}\right) .
$$

If there are no variations in density or composition, the medium can be treated as homogeneous in nature. Then the susceptibility tensor $\chi$ will not depend on $r$ or $\boldsymbol{r}^{\prime}$ separately but only on the spatial difference $\boldsymbol{r}-\boldsymbol{r}^{\prime}$. A second simplification can be exploited. If the electric field exhibits only a slow variation in space and time we may use $\boldsymbol{E}\left(\boldsymbol{r}^{\prime}, t^{\prime}\right) \approx \boldsymbol{E}(\boldsymbol{r}, t)$ and recover Eq. (3.9a), where we now have shown the structure of the susceptibility tensor in more detail:

$$
P_{\alpha}^{(L)}(\mathbf{r}, t)=\sum_{\beta} \chi_{\alpha \beta}^{(1)}(\boldsymbol{r}, t) E_{\beta}(\boldsymbol{r}, t), \quad \text { with } \quad \chi_{\alpha \beta}^{(1)}(\boldsymbol{r}, t)=\int d^{3} r^{\prime} d t^{\prime} \chi_{\alpha \beta}\left(\boldsymbol{r}-\boldsymbol{r}^{\prime}, t-t^{\prime}\right)
$$

The physical meaning of nonlinear response of a material becomes clear if analyzed in Fourier space. Therefore we will briefly list the basic Fourier decompositions, again exemplified for linear response. For simplicity we will use the same symbols for functions in Fourier as well as in real space and follow the notation in [75]. The Fourier transform operator and its inverse are denoted by the symbols $\mathcal{F}$ and $\mathcal{F}^{-1}$, respectively.

$$
\begin{gathered}
E_{\beta}(\boldsymbol{k}, \omega)=\mathcal{F}\left[E_{\beta}(\boldsymbol{k}, \omega)\right] \equiv \int d^{3} r d t E_{\beta}(\boldsymbol{r}, t) e^{-i \boldsymbol{k} \cdot \boldsymbol{r}} e^{i \omega t} \\
E_{\beta}(\boldsymbol{r}, t)=\mathcal{F}^{-1}\left[E_{\beta}(\boldsymbol{k}, \omega)\right] \equiv \int \frac{d^{3} k d \omega}{(2 \pi)^{4}} E_{\beta}(\boldsymbol{k}, \omega) e^{i \boldsymbol{k} \cdot \boldsymbol{r}} e^{-i \omega t} .
\end{gathered}
$$


With these definitions the transformation for $P_{\alpha}^{(L)}$ follows directly from Eq. (3.11):

$$
P_{\alpha}^{(L)}(\boldsymbol{r}, t)=\int \frac{d^{3} k d \omega}{(2 \pi)^{4}} P_{\alpha}^{(L)}(\boldsymbol{k}, \omega) e^{i \boldsymbol{k} \cdot \boldsymbol{r}} e^{-i \omega t}
$$

where

$P_{\alpha}^{(L)}(\boldsymbol{k}, \omega)=\sum_{\beta} \chi_{\alpha \beta}^{(1)}(\boldsymbol{k}, \omega) E_{\beta}(\boldsymbol{k}, \omega), \quad$ and $\quad \chi_{\alpha \beta}^{(1)}(\boldsymbol{k}, \omega)=\int d^{3} r d t \chi_{\alpha \beta}^{(1)}(\boldsymbol{r}, t) e^{-i \boldsymbol{k} \cdot \boldsymbol{r}} e^{i \omega t}$.

For the rest of this chapter we will stick to the linear response and assume the simple linear proportionality between $\boldsymbol{P}$ and $\boldsymbol{E}$. Nonlinear optical responses will again be discussed in Chap. 7.

\subsubsection{Electromagnetic potentials}

We now return to Maxwell's equations. Let us recall their appearance in a source free frequency space:

$$
\begin{array}{lll}
\boldsymbol{\nabla} \cdot \boldsymbol{D}(\boldsymbol{r}, \omega)=0, & \boldsymbol{\nabla} \times \boldsymbol{B}(\boldsymbol{r}, \omega)+i \omega \boldsymbol{D}(\boldsymbol{r}, \omega)=0, \\
\boldsymbol{\nabla} \cdot \boldsymbol{B}(\boldsymbol{r}, \omega)=0, & \boldsymbol{\nabla} \times \boldsymbol{E}(\boldsymbol{r}, \omega)-i \omega \boldsymbol{B}(\boldsymbol{r}, \omega)=0 .
\end{array}
$$

Taking the curl on Ampère's and Faraday's law respectively and substituting the corresponding equations leads us to the wave equation of Helmholtz form:

Wave equation for electromagnetic fields

$$
\left(\nabla^{2}+\varepsilon \frac{\omega^{2}}{c^{2}}\right)\left\{\begin{array}{l}
\boldsymbol{E}(\boldsymbol{r}, \omega) \\
\boldsymbol{B}(\boldsymbol{r}, \omega)
\end{array}\right\}=\mathbf{0}
$$


This equation is a central result of Maxwell's theory, since it postulates the existence of electromagnetic waves. Thus as a possible solution we can write down a plane wave propagating in $\hat{\boldsymbol{e}}_{r}$-direction: $e^{i \boldsymbol{k} \cdot \boldsymbol{r}-i \omega t}$, with $\boldsymbol{k}=k \hat{\boldsymbol{e}}_{k}$.

If we change from first to second order equations, we can combine the four coupled expressions of Eq. (3.15) into two new equations and introduce the vector potential $\boldsymbol{A}$ and the scalar potential $\Phi$. The fields can then be expressed as

\section{Electromagnetic fields expressed with potentials}

$$
\begin{aligned}
& \boldsymbol{B}=\boldsymbol{\nabla} \times \boldsymbol{A}, \\
& \boldsymbol{E}=-\nabla \Phi+i k \boldsymbol{A} .
\end{aligned}
$$

The differential equations for $\boldsymbol{A}$ and $\Phi$ still form a coupled system. But because of the gauge invariance of the potentials we can choose $\boldsymbol{A}$ and $\Phi$ in such a way that they fulfill the Lorenz condition [79]

$$
\boldsymbol{\nabla} \cdot \boldsymbol{A}-i k \varepsilon \Phi=0
$$

This condition does not entirely fix the gauge, but it is coordinate independent (and therefore naturally fits into special relativity) and leads to two decoupled wave equations for $\boldsymbol{A}$ and $\Phi$ that are completely equivalent to Maxwell's equations (3.1):

\section{Helmholtz equation for potentials}

$$
\begin{aligned}
\nabla^{2} \Phi+k^{2} \varepsilon \Phi & =-4 \pi \varrho, \\
\nabla^{2} \boldsymbol{A}+k^{2} \varepsilon \boldsymbol{A} & =-\frac{4 \pi}{c} \boldsymbol{j} .
\end{aligned}
$$

Here we again included external charges and currents as in (3.1). 


\section{Additional remark}

Maxwell's equations interweave space and time, electric and magnetic fields in such a wonderful way, that made Boltzmann express his deepest admiration [67]. Throughout time the equations changed their appearance and hence are a nice example for the mathematical beauty and the huge amount of physics that can be contained in one single line (constants set to 1 , table adopted from [67]):

\section{Homogeneous equations}

\section{Inhomogeneous equations}

Original form:

$$
\begin{array}{ll}
\frac{\partial B_{x}}{\partial x}+\frac{\partial B_{y}}{\partial y}+\frac{\partial B_{z}}{\partial z}=0, & \frac{\partial E_{x}}{\partial x}+\frac{\partial E_{y}}{\partial y}+\frac{\partial E_{z}}{\partial z}=\varrho, \\
\frac{\partial E_{z}}{\partial y}-\frac{\partial E_{y}}{\partial z}=-\frac{\partial B_{x}}{\partial t} & \frac{\partial B_{z}}{\partial y}-\frac{\partial B_{y}}{\partial z}=j_{x}+\frac{\partial E_{x}}{\partial t} \\
\frac{\partial E_{x}}{\partial z}-\frac{\partial E_{z}}{\partial x}=-\frac{\partial B_{y}}{\partial t} & \frac{\partial B_{x}}{\partial z}-\frac{\partial B_{z}}{\partial x}=j_{y}+\frac{\partial E_{y}}{\partial t} \\
\frac{\partial E_{y}}{\partial x}-\frac{\partial E_{x}}{\partial y}=-\frac{\partial B_{z}}{\partial t} & \frac{\partial B_{y}}{\partial x}-\frac{\partial B_{x}}{\partial y}=j_{z}+\frac{\partial E_{z}}{\partial t}
\end{array}
$$

End of 19th century:

$$
\begin{array}{rlrl}
\operatorname{div} \boldsymbol{B} & =0 & \operatorname{div} \boldsymbol{E}=\varrho \\
\operatorname{rot} \boldsymbol{E}=-\dot{\boldsymbol{B}} & \operatorname{rot} \boldsymbol{B}=\boldsymbol{j}+\dot{\boldsymbol{E}}
\end{array}
$$

Beginning of 20th century:

$$
* F_{, \alpha}^{\beta \alpha}=0 \quad F_{, \alpha}^{\beta \alpha}=j^{\beta}
$$

Mid of 20th century:

$$
d F=0
$$

$\delta F=J$ 


\subsection{Rayleigh scattering - The quasistatic approximation}

Before we discuss the solution of the full wave equations in the next section, let us elucidate the main idea for a more simplified case. In the limit of small particles (compared to the wavelength $\lambda$ ) we can put $k \approx 0$ and neglect all retardation effects. The wave equation (3.19a) for the scalar potential $\Phi$ and a non vanishing external charge distribution $\varrho$ transforms to the Poisson equation

$$
\nabla^{2} \Phi(\boldsymbol{r})=-4 \pi \varrho(\boldsymbol{r})
$$

If there are again no external charges present, this equation reduces to the Laplace equation

$$
\nabla^{2} \Phi(\boldsymbol{r})=0
$$

Because of an almost 200 years old work [80] from the extraordinary autodidact George Green ${ }^{10}$, we know that we can solve this kind of differential equations with the introduction of the Green function $G$

$$
\nabla^{2} G\left(\boldsymbol{r}-\boldsymbol{r}^{\prime}\right)=-4 \pi \delta\left(\boldsymbol{r}-\boldsymbol{r}^{\prime}\right)
$$

With the Dirac delta distribution on the right hand side, we already get a first hint about the singularity of $G$. In the quasistatic regime the solution of Eq. (3.22) is

\section{Quasistatic Green function}

$$
G\left(\boldsymbol{r}-\boldsymbol{r}^{\prime}\right)=\frac{1}{\left|\boldsymbol{r}-\boldsymbol{r}^{\prime}\right|}=G\left(\boldsymbol{r}, \boldsymbol{r}^{\prime}\right)
$$

plus an arbitrary function which has to obey the Laplace equation [66]. With this static Green function $G$ we are now prepared to solve the Poisson equation within an

${ }^{10}$ Born $14^{\text {th }}$ July 1793 in Sneinton, Nottingham; $† 31^{\text {st }}$ May 1841 in Nottingham. See e.g. [81] for more details on his life. 
unbounded region, simply by applying Green's theorem together with appropriate Dirichlet or Neumann boundary conditions, see [66] for more details.

Let us assume a given external excitation $\phi_{\text {ext }}$, which, for example, corresponds to plane waves impinging on a nanoparticle. The only possible sources are bound to the nanoparticle surface $\partial \Omega$, therefore the scalar potential can be expressed as $[82,83]$

$$
\phi(\boldsymbol{r})=\int_{\partial \Omega} G\left(\boldsymbol{r}-\boldsymbol{s}^{\prime}\right) \sigma\left(\boldsymbol{s}^{\prime}\right) d a^{\prime}+\phi_{\mathrm{ext}}(\boldsymbol{r})
$$

with $\sigma$ being the surface charge distribution situated at the interface $\partial \Omega$ between the two media. Note that this equation fulfills the Poisson equation everywhere except at the boundaries.

We make an important step here, that highlights the main idea of our approach in the quasistatic regime: For an unbounded region we can write down the solutions of the Poisson or Laplace equation quite easily. For a bounded region the corresponding boundary conditions come into play and we add artificially a surface charge distribution that will be chosen such that the boundary constraints are fulfilled.

The electromagnetic boundary conditions are discussed in App. A.1: The tangential electric field and the normal component of the dielectric displacement have to be continuous at the boundary between two media. The first constraint implies that the scalar potential is continuous $\left.\Phi_{1}\right|_{\text {surf }}=\left.\Phi_{2}\right|_{\text {surf }}$, which is guaranteed when the surface charge distribution is the same on each side of the boundary: $\sigma_{1}=\sigma_{2}$.

For the second constraint we have to evaluate the surface derivative of $\Phi$

$$
\lim _{\boldsymbol{r} \rightarrow \boldsymbol{s}} \hat{\boldsymbol{n}} \cdot \nabla \Phi(\boldsymbol{r}) \equiv \lim _{\boldsymbol{r} \rightarrow \boldsymbol{s}} \frac{\partial \Phi(\boldsymbol{r})}{\partial n}=\lim _{\boldsymbol{r} \rightarrow \boldsymbol{s}}\left\{\frac{\partial}{\partial n} \int_{\partial \Omega} G\left(\boldsymbol{r}-\boldsymbol{s}^{\prime}\right) \sigma\left(\boldsymbol{s}^{\prime}\right) d a^{\prime}+\frac{\partial \phi_{\mathrm{ext}}(\boldsymbol{r})}{\partial n}\right\} .
$$

Because of the singularity of $G$ we have to be careful about the limit $r \rightarrow s$ in the integral. Let us consider

$$
\lim _{r \rightarrow s} \hat{\boldsymbol{n}} \cdot \nabla \int G\left(\boldsymbol{r}, \boldsymbol{s}^{\prime}\right) \sigma\left(\boldsymbol{s}^{\prime}\right) d s^{\prime}
$$


for a coordinate system where $\hat{\boldsymbol{n}}=\hat{\boldsymbol{e}}_{z}, \boldsymbol{r}=(0,0, z)^{T}$, and $\boldsymbol{s}^{\prime}=\rho(\cos \varphi, \sin \varphi, 0)^{T}$ is given in polar coordinates $\rho$ and $\varphi$, see [56]. We compute the boundary integral within a small circle with radius $R$, within which the surface charge $\sigma$ can be approximated by a constant. The integral then becomes

$$
\lim _{z \rightarrow \pm 0} \hat{\boldsymbol{n}} \cdot \int \frac{\boldsymbol{r}-\boldsymbol{s}^{\prime}}{\left|\boldsymbol{r}-\boldsymbol{s}^{\prime}\right|^{3}} d s^{\prime} \rightarrow \lim _{z \rightarrow \pm 0} 2 \pi z \int_{0}^{R} \rho d \rho\left(\rho^{2}+z^{2}\right)^{-\frac{3}{2}}= \pm 2 \pi .
$$

The positive or negative sign depends on the direction from where we approach the surface, whether from inside or outside of the particle boundary (i.e. positive sign inside the particle, negative sign outside of it). With the abbreviation $F(s$, $\left.\boldsymbol{s}^{\prime}\right)=(\hat{\boldsymbol{n}} \cdot \boldsymbol{\nabla}) G\left(\boldsymbol{s}, \boldsymbol{s}^{\prime}\right)$ we finally obtain

$$
\lim _{\boldsymbol{r} \rightarrow \boldsymbol{s}}(\hat{\boldsymbol{n}} \cdot \nabla) \Phi(\boldsymbol{r})=\frac{\partial \Phi(\boldsymbol{s})}{\partial n}=\int_{\partial \Omega} F\left(\boldsymbol{s}, \boldsymbol{s}^{\prime}\right) \sigma\left(\boldsymbol{s}^{\prime}\right) d s^{\prime} \pm 2 \pi \sigma(\boldsymbol{s})+\frac{\partial \Phi_{\mathrm{ext}}(\boldsymbol{s})}{\partial n} .
$$

\subsubsection{From boundary integrals to boundary elements}

The calculation of an analytical solution of Eq. (3.27) is only possible for very restricted geometries, for example if $\partial \Omega$ is a sphere boundary. In Chap. 4 different numerical methods and approaches will be discussed, to get solutions for more general shapes of $\partial \Omega$. The method that has been used throughout this work is the so called Boundary Element Method (BEM). In this approach one approximates the surface charge as a discrete number of points located at the centroids of small surface elements (see Sec. 4.3 for details). Eq. (3.27) then becomes

$$
\left(\frac{\partial \Phi}{\partial n}\right)_{i}=\sum_{j} F_{i j} \sigma_{j} \pm 2 \pi \sigma_{i}+\left(\frac{\partial \Phi_{\mathrm{ext}}}{\partial n}\right)_{i}
$$

or in a compact matrix notation 


$$
\frac{\partial \Phi}{\partial n}=\boldsymbol{F} \sigma \pm 2 \pi \sigma+\frac{\partial \Phi_{\mathrm{ext}}}{\partial n}
$$

where $\boldsymbol{F}$ is the matrix with elements $F\left(s_{i}, \boldsymbol{s}_{j}\right)$.

So far so good, but we still have to account for Maxwell's boundary conditions. The continuity of the normal component of the dielectric displacement leads to

$$
\begin{aligned}
\varepsilon_{2}\left(F \sigma+2 \pi \sigma+\frac{\partial \Phi_{\text {ext }}}{\partial n}\right) & =\varepsilon_{1}\left(F \sigma-2 \pi \sigma+\frac{\partial \Phi_{\text {ext }}}{\partial n}\right) \\
2 \pi \sigma\left(\varepsilon_{2}+\varepsilon_{1}\right)+F \sigma\left(\varepsilon_{2}+\varepsilon_{1}\right) & =-\left(\varepsilon_{2}-\varepsilon_{1}\right) \frac{\partial \Phi_{\text {ext }}}{\partial n}
\end{aligned}
$$

where $\varepsilon_{1,2}$ is the (frequency dependent) dielectric function of the corresponding medium, see Fig. 2.2. From the last expression it follows that

$$
\sigma=-\left\{2 \pi \frac{\epsilon_{2}+\epsilon_{1}}{\epsilon_{2}-\epsilon_{1}} \mathbb{1}+\boldsymbol{F}\right\}^{-1} \frac{\partial \Phi_{\mathrm{ext}}}{\partial n} .
$$

With the abbreviation $\Lambda=2 \pi \frac{\varepsilon_{2}+\varepsilon_{1}}{\varepsilon_{2}-\varepsilon_{1}}$ we get our final result

\section{Quasistatic surface charge}

$$
\sigma=-(\boldsymbol{\Lambda}+\boldsymbol{F})^{-1} \frac{\partial \Phi_{\mathrm{ext}}}{\partial n}
$$

Note the elegance of this expression, where material parameters (incorporated by the frequency dependent $\boldsymbol{\Lambda}$ ) and structural properties (established by $\boldsymbol{F}$ ) of the problem are fully decoupled.

\subsubsection{Eigenmode expansion}

To compute the surface charge $\sigma$ according to Eq. (3.31) we have to perform a matrix inversion. The size of the matrix (i.e. the refinement of our surface discretization) 
is the limiting parameter for the needed computation time (see Sec. C.1). In the quasistatic regime we can significantly speed up the simulation if we expand Eq. (3.31) to plasmon eigenmodes and reduce the inversion of the fully populated matrix to a much faster inversion of a diagonal matrix. Additionally it turns out that we do not need a complete set of eigenmodes to get very accurate results, see [84].

At first we define left and right eigenvectors $\sigma_{k}^{L}$ and $\sigma_{k}^{R}$ of the surface derivative of the Green function through $[85,86]$

$$
\left\langle\boldsymbol{F}, \sigma_{k}^{R}\right\rangle=\lambda_{k} \sigma_{k}^{R}, \quad\left\langle\sigma_{k}^{L}, \boldsymbol{F}\right\rangle=\lambda_{k} \sigma_{k}^{L}
$$

which form a biorthogonal set with $\left\langle\sigma_{k}^{L}, \sigma_{k^{\prime}}^{R}\right\rangle=\left\langle\sigma_{k}^{R}, \sigma_{k^{\prime}}^{L}\right\rangle=\delta_{k k^{\prime}}$. The inner product is defined through a surface integration:

$$
\langle A, B\rangle=\int_{\partial \Omega} A(s) B(s) d s .
$$

Note that $\boldsymbol{F}$ is a non-Hermitian matrix, but with real eigenvalues, consult $[85,87]$ for further details.

The functions $\sigma_{k}^{R}$ can be interpreted as the surface plasmon eigenmodes, and the response to any external perturbation can be decomposed into these modes viz.

$$
\left\langle\sigma_{k}^{L},(\boldsymbol{\Lambda}+\boldsymbol{F})\right\rangle \sigma=\left(\boldsymbol{\Lambda}+\lambda_{k}\right) \sigma_{k}^{L} \sigma=-\left\langle\sigma_{k}^{L}, \frac{\partial \phi_{\mathrm{ext}}}{\partial n}\right\rangle,
$$

which leads to

\section{Quasistatic surface charge eigenmode expansion}

$$
\sigma=-\sum_{k} \frac{\sigma_{k}}{\Lambda(\omega)+\lambda_{k}}\left\langle\sigma_{k}^{L}, \frac{\partial \phi_{\mathrm{ext}}}{\partial n}\right\rangle
$$

It is apparent that a given mode $k$ gives a noticeable contribution only if the coupling $\left\langle\sigma_{k}^{L}, \frac{\partial \phi_{\text {ext }}}{\partial n}\right\rangle$ to the external potential is sufficiently strong and if the denominator 
becomes small - this second requirement brings us directly to the plasmon resonance condition that connects our calculated eigenenergy $\lambda_{k}$ with the actual photonic energy $\omega:$

$$
\operatorname{Re}\left[\Lambda(\omega)+\lambda_{k}\right]=0
$$

We have to be careful here, because $\boldsymbol{\Lambda}$ is a complex quantity and we assume that the spectral variation of the imaginary part is sufficiently small. For gold particles, this is only true if $\omega$ is far away from the d-band absorption. For small gold nanospheres Eq. (3.36) will therefore not give the proper resonance frequency!
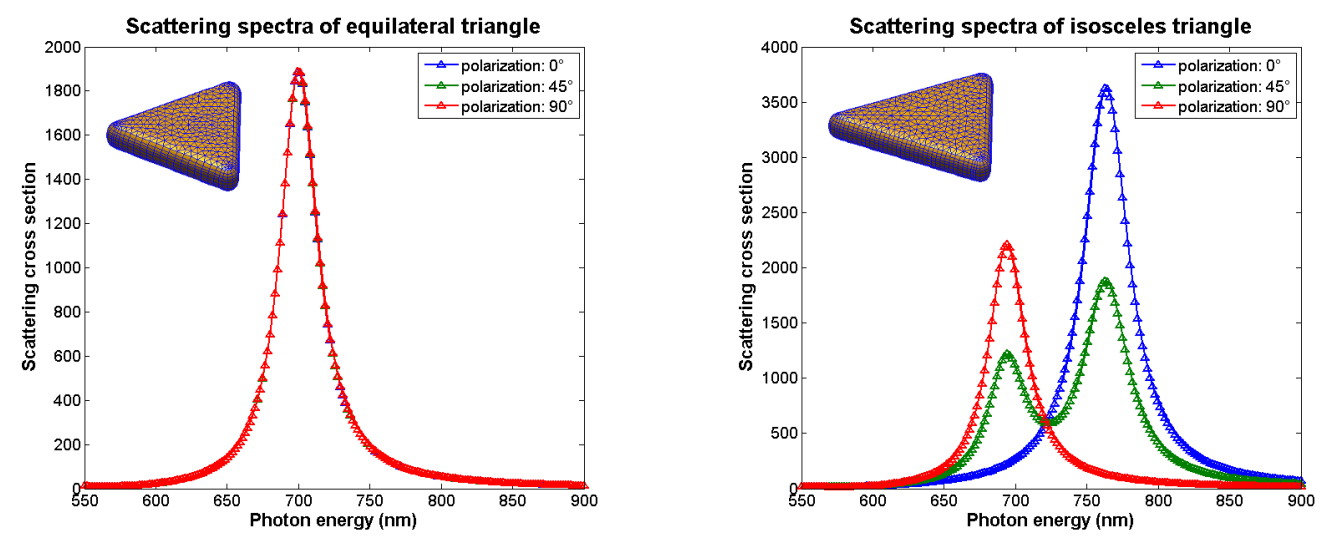

Figure 3.1.: Scattering spectra of an equilateral (left hand side) and an isosceles (right hand side) gold nanotriangle with three different polarization angles. The basis edge length of the triangles is $50 \mathrm{~nm}$ and the isosceles triangle has an altitude of $55 \mathrm{~nm}$. The dielectric surrounding is modeled with an effective refraction index $n_{b}=1.33$.

Nevertheless the eigenmode expansion is very useful for the physical interpretation of optical phenomena of nanoparticles and allows new insight on the behavior of plasmons. A very nice illustration of the convenience of eigenmodes is the optical spectrum of an equilateral triangle. If we illuminate such a triangle with polarized light, at first it is a little bit surprising that we do not find any polarization dependence at all in contrast to spectra of other triangles, see Fig. 3.1. Plotting the surface charge at the resonance energy of $700 \mathrm{~nm}$ of the equilateral triangle in Fig. 3.1 gives 
us no hint about its polarization independence, instead we see the expected behavior plotted in Fig. 3.2.
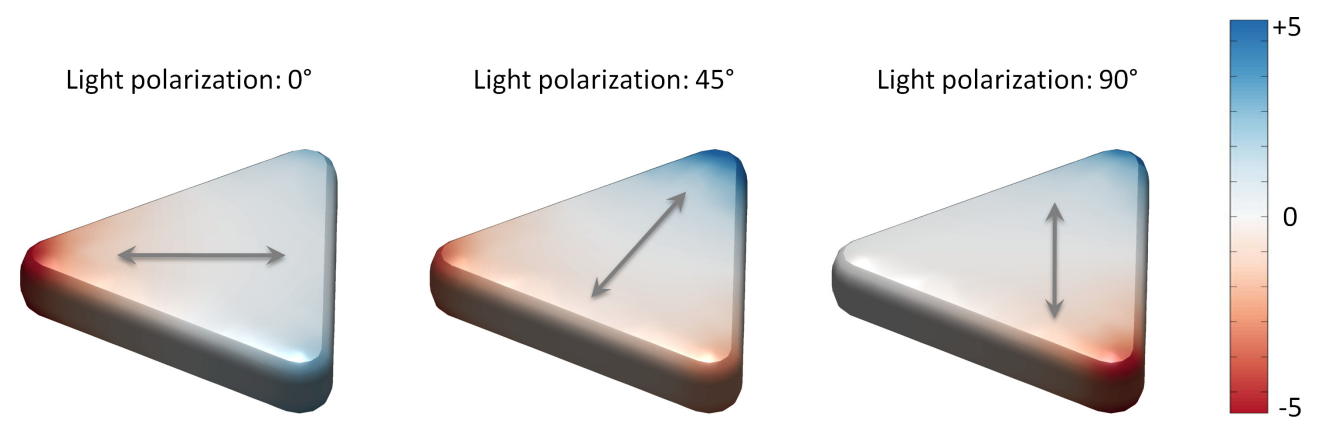

Figure 3.2.: Surface charge in arbitrary units at the resonance energy of $688.8 \mathrm{~nm}$ for an equilateral triangle with $50 \mathrm{~nm}$ edge length and a height of $10 \mathrm{~nm}$. The particle is illuminated from the top.

The calculation of the eigenmodes on the other hand shows us very quickly, that for an equilateral triangle several eigenmodes are degenerate, whereas (because of the broken symmetry) there is no degeneracy for an isosceles triangle, see Fig 3.3 and Fig. 3.5.
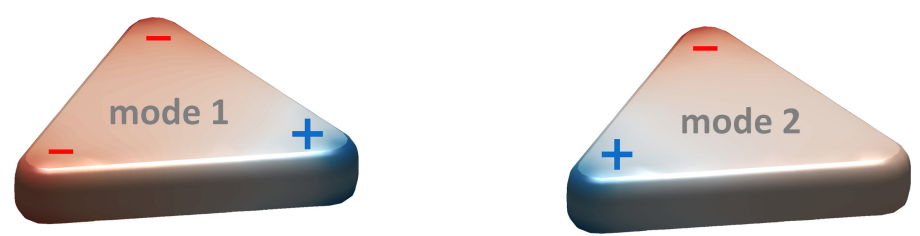

Figure 3.3.: The first two (degenerate) dipolar eigenmodes of an equilateral triangle with edge length $50 \mathrm{~nm}$ and a height of $10 \mathrm{~nm}$.

Thus, for equilateral triangles the surface charge distribution in Fig. 3.2 can be generated through a linear combination of the first two optical active and degenerated eigenmodes plotted in Fig. 3.3, yielding always the same peak position independent of the polarization angle. In Fig. 3.4 we show an example for the surface charge distribution with polarization along the $\mathrm{x}$-axis. 


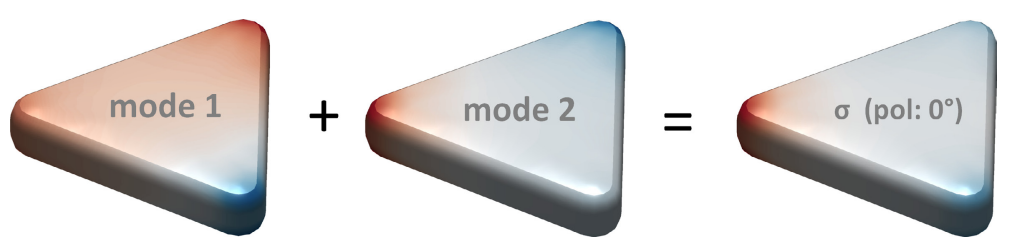

Figure 3.4.: The linear combination of the first two (degenerate) dipolar eigenmodes of an equilateral triangle generates the surface charge distribution plotted in Fig. 3.2.

The corresponding coefficients of the linear combination can be calculated from $c_{k}=$ $\left\langle\sigma_{k}^{L}, \sigma\right\rangle$ and show again, that the first two eigenmodes are dominating the surface charge distribution.

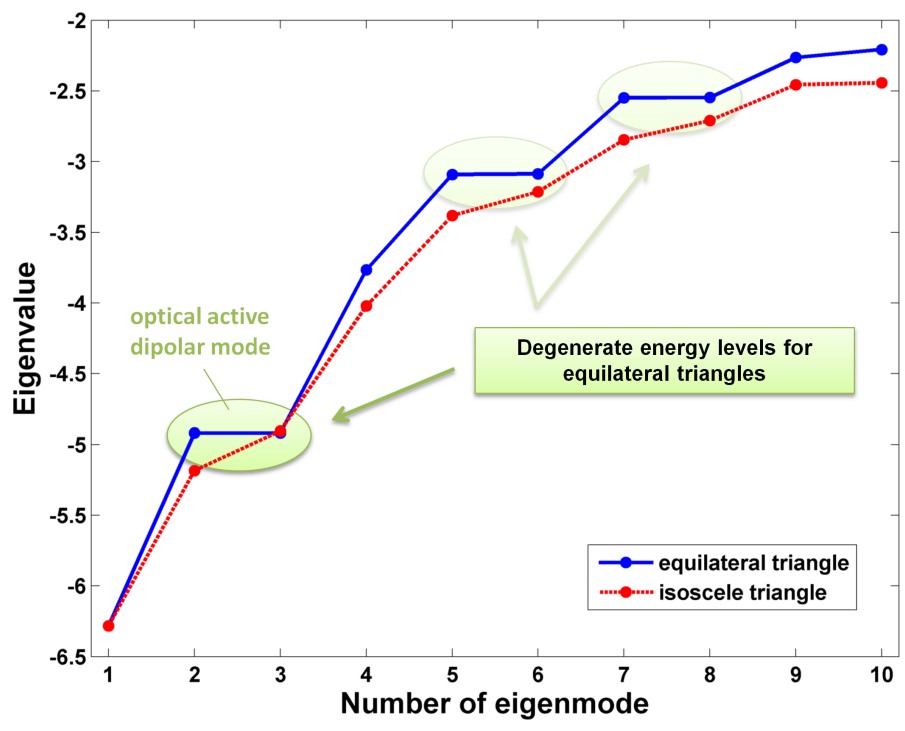

Figure 3.5.: The eigenenergies $\lambda_{k}$ of the first ten eigenmodes for an for an equilateral and isosceles triangle with horizontal light polarization, see Eq. (3.35) and Eq. (3.36). 


\subsection{Solving the full Maxwell equations}

In the last section we made the assumption, that the characteristic length of the investigated system is much smaller than the wavelength of light. For bigger particles or structures this approximation becomes questionable and we have to solve Maxwell equations in their full glory.

Let us first note the main impacts of $k \neq 0$ : The vector potential $\boldsymbol{A}$ does not vanish anymore and the electromagnetic fields remain in the form of Eq. (3.17)

$$
\mathbf{E}=i k \mathbf{A}-\nabla \phi, \quad \mathbf{B}=\nabla \times \mathbf{A} .
$$

As already discussed in section 3.2 we will apply the Lorenz gauge Eq. (3.18)

$$
\boldsymbol{\nabla} \cdot \boldsymbol{A}-i k \varepsilon \Phi=0
$$

This allows us in principle to consider only the vector potential ${ }^{11}$ and to express the scalar one through Eq. (3.18). Nevertheless, in the following we keep both potentials and exploit a scheme [84] where we only need the Green function and it's surface derivative (in the interest of readability we use the same symbol $G$ as in the quasistatic approach). The retarded Green function now has to obey

$$
\left[\nabla^{2}+k_{j}^{2}\right] G_{j}(r)=-4 \pi \delta(\boldsymbol{r})
$$

The solution to this equation is given by

\section{Retarded Green function and surface derivative}

$$
G_{j}=\frac{e^{i k_{j}\left|\boldsymbol{r}-\boldsymbol{r}^{\prime}\right|}}{\left|\boldsymbol{r}-\boldsymbol{r}^{\prime}\right|}, \quad F_{j}=\hat{\boldsymbol{n}}_{s} \cdot \nabla_{s} G_{j}\left(\left|\boldsymbol{s}-\boldsymbol{s}^{\prime}\right|\right),
$$

\footnotetext{
${ }^{11}$ It is also possible to establish a numerical approach based on the electromagnetic fields instead of the potentials. But in contrast to our simple collocation scheme, see Sec. 4.3, this usually requires more complex numerical implementations. In the potential-based BEM approach we have to invert matrices of the order $N \times N$, whereas in the field-based BEM approach the matrices are of the order $3 N \times 3 N$.
} 
where the subscript $j$ indicates the medium. Accordingly the wave number $k_{j}$ follows as

$$
k_{j}=k \sqrt{\varepsilon_{j} \mu_{j}}=k \sqrt{\varepsilon_{j}}
$$

where the square root is understood to yield positive imaginary parts (this sign choice ensures that the potentials vanish at infinity, while at the same time, it is consistent with the retarded response formalism) [82].

Armed with $G$ and its surface derivative $F$ we are now well prepared to solve Eq. (3.19) inside each medium $j$ analogous to the quasistatic case:

$$
\begin{aligned}
& \phi(\boldsymbol{r})=\int_{\partial \Omega_{j}} G_{j}\left(\boldsymbol{r}-\boldsymbol{s}^{\prime}\right) \sigma_{j}\left(\boldsymbol{s}^{\prime}\right) d a^{\prime}+\phi_{\mathrm{ext}}(\boldsymbol{r}) \\
& \boldsymbol{A}(\boldsymbol{r})=\int_{\partial \Omega_{j}} G_{j}\left(\boldsymbol{r}-\boldsymbol{s}^{\prime}\right) \boldsymbol{h}_{j}\left(\boldsymbol{s}^{\prime}\right) d a^{\prime}+\boldsymbol{A}_{\mathrm{ext}}(\boldsymbol{r}) .
\end{aligned}
$$

Up to now everything was very similar to the quasistatic approach and the solutions above automatically fulfill the Helmholtz equations within the different media again. But there are two major differences compared to the quasistatic regime [84]: Identical surface charges and currents at different boundary sides are in general no longer possible and we have to chose the external potentials inside the different regions $j$ such that they are only induced by sources neighboring the particle boundaries directly (and are sitting on the correct boundary side), see Fig. 3.6.

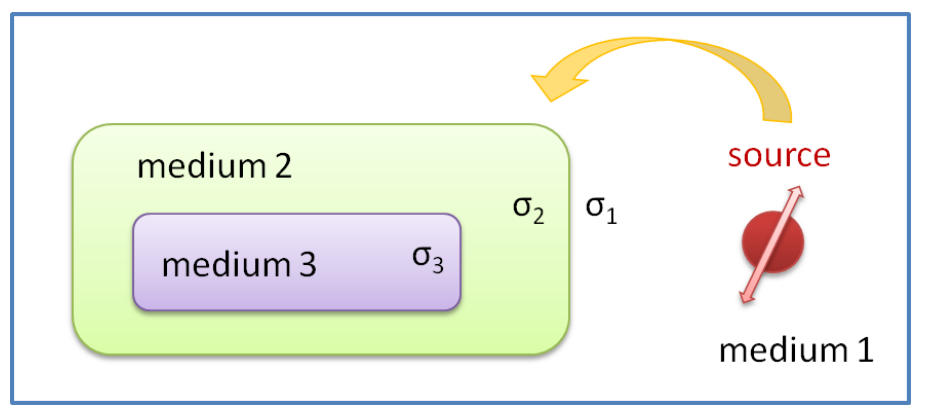

Figure 3.6.: A source in medium 1 can only excite the outer boundary of medium 2 , but neither the inner boundary of medium 2 nor the boundaries of medium 3 . Also $\sigma_{1} \neq \sigma_{2} \neq \sigma_{3}$ 


\subsubsection{Boundary conditions}

Similar to Eq. (3.24) we once again wrote down a solution for the potentials in terms of auxiliary surface charges and currents [Eq. (3.40)], which can be calculated from Maxwell's boundary conditions. Since the expressions are a little bit more laborious than in the quasistatic case, it becomes handy to introduce shorthand notations. By following [84] and [88] we will use the notation hereafter for the scalar potentials inside and outside the particle boundaries:

$$
\begin{aligned}
& \Phi_{1}=G_{11} \sigma_{1}+G_{12} \sigma_{2}+\phi_{1}^{\mathrm{ext}} \\
& \Phi_{2}=G_{21} \sigma_{1}+G_{22} \sigma_{2}+\phi_{2}^{\text {ext }} .
\end{aligned}
$$

The following expressions Eq. (3.42) - Eq. (A.19) are the central part of the fully retarded BEM approach. Let us now exploit the boundary conditions discussed in App. A.1. The continuity of the potential can be expressed as

$$
\begin{aligned}
G_{1} \sigma_{1} & =G_{2} \sigma_{2}+\Delta \phi_{\mathrm{ext}}, & G_{1} \boldsymbol{h}_{1} & =G_{2} \boldsymbol{h}_{2}+\Delta \boldsymbol{A}_{\mathrm{ext}} \\
\sigma_{1} & =G_{1}^{-1}\left(G_{2} \sigma_{2}+\Delta \phi_{\mathrm{ext}}\right), & \boldsymbol{h}_{1} & =G_{1}^{-1}\left(G_{2} \boldsymbol{h}_{2}+\Delta \boldsymbol{A}_{\mathrm{ext}}\right),
\end{aligned}
$$

where we have used

$$
\begin{aligned}
G_{1} & =G_{11}-G_{21}, & \Delta \phi_{\text {ext }} & =\phi_{2}^{\text {ext }}-\phi_{1}^{\text {ext }}, \\
G_{2} & =G_{22}-G_{12}, & \Delta \boldsymbol{A}_{\text {ext }} & =\boldsymbol{A}_{2}^{\text {ext }}-\boldsymbol{A}_{1}^{\text {ext }} .
\end{aligned}
$$

Note that Eq. (3.43) shows us one of the differences to the quasistatic expression mentioned above - the surface charge (and current) on the inside and outside is not identical but rather related through this equation.

\subsubsection{Surface charges and currents}

In the next step we have to account for the boundary conditions. Since the calculation is somewhat bulky, we refer to App. A.4 and present the surface charges and currents for the fully retarded regime without further fanfare: 


\section{Retarded surface charge and current}

$$
\begin{array}{lll}
\sigma_{2}=G_{2}^{-1} \Sigma^{-1}\left[D_{e}+i k \hat{\boldsymbol{n}}\left(L_{1}-L_{2}\right) \Delta^{-1} \vec{\alpha}\right], & \sigma_{1}=G_{1}^{-1}\left(G_{2} \sigma_{2}+\Delta \phi_{\mathrm{ext}}\right), \\
\boldsymbol{h}_{2}=G_{2}^{-1} \Delta^{-1}\left[i k \hat{\boldsymbol{n}}\left(L_{1}-L_{2}\right) G_{2} \sigma_{2}+\vec{\alpha}\right], & \boldsymbol{h}_{1}=G_{1}^{-1}\left(G_{2} \boldsymbol{h}_{2}+\Delta \boldsymbol{A}_{\mathrm{ext}}\right)
\end{array}
$$

The corresponding abbreviations used in Eq. (3.44) can again be found in App. A.4. In Fig. 3.7 the surface charge and current density for a gold nanotriangle is exemplified.
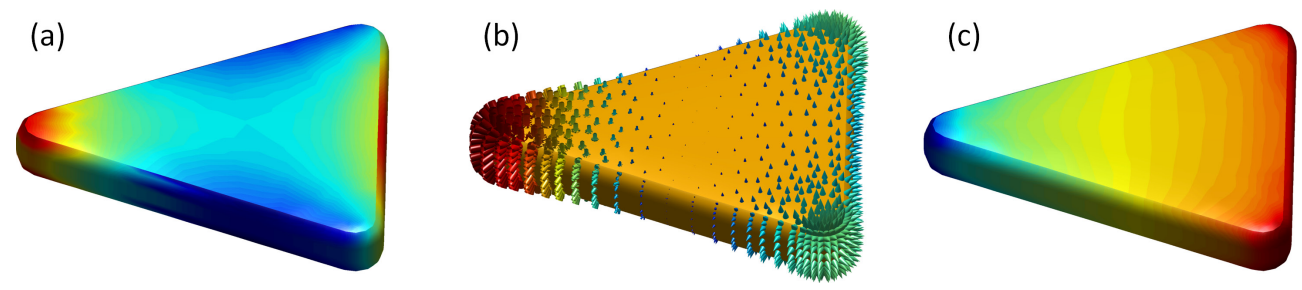

Figure 3.7.: (a) Surface charge density $\sigma_{2}$ of a gold nanotriangle $\left(55 \times 50 \times 8 \mathrm{~nm}, n_{b}\right.$ $=1.34$ ) at the resonance energy of $792 \mathrm{~nm}$, also see Fig. 4.4. (b) Surface current density $\boldsymbol{h}_{2}$ again at the resonance and (c) surface charge density at $1200 \mathrm{~nm}$. 



\title{
4. Modeling the optical response of metallic nanoparticles
}

\begin{abstract}
A formal solution of rigorous scattering theory for nanoparticles is unfortunately only possible for restricted geometries. There exist analytical solutions for light scattering problems if we expand the electromagnetic potentials and fields to spherical harmonics and limit ourselves to spherical or spheroidal particle shapes. In reality, however, we want to work with arbitrary shaped nanostructures and take advantage of certain structure dependent qualities like the hot spots in the gap regions of bowtie antennas or the magnetic response of split-ring resonators. So in general a more sophisticated numerical method for solving Maxwell's equations is essential and inevitable. Several different techniques are available and some of them will be discussed in the next sections.
\end{abstract}

A critical comparison of the capabilities of the most popular and efficient approaches can be found in [60], for example, an overview is also presented at the end of this chapter. One approximation that all of these methods have in common is the locality of the dielectric description of the material, i.e. the dielectric function depends only on the frequency of light $\varepsilon\left(\boldsymbol{r}, \boldsymbol{r}^{\prime}, \omega\right) \approx \varepsilon(\omega)$ and bodies with abrupt interfaces are assumed. This is a valid assumption as long as the studied structures are sufficiently large and finite size effects can be neglected, see Sec. 4.5.3 for more details.

The optical theorem ${ }^{1}$ relates the forward scattering amplitude to the total cross section of the scatterer, and thus allows us to determine the optical response of MNPs.

${ }^{1}$ Consult [66] for a derivation and discussion of the theorem, also see [89]. 


\subsection{Analytic solutions}

\subsubsection{Quasistatic approximation - Rayleigh theory}

Let us start with the quasistatic regime, a topic that has already been discussed in Sec. 3.3. If the investigated structures have spatial dimensions below say $50 \mathrm{~nm}$, we can connect the macroscopic dielectric function with the microscopic polarizability $\alpha$. The elastic scattering of light can then be described in terms of Rayleigh scattering.

For a microscopic derivation of $\alpha$ consult [39], for example. Expressing the dipole moment $\boldsymbol{P}$ through the local microscopic electric field on one hand, and connecting it with the dielectric function $\varepsilon$ (through $\boldsymbol{D}=\varepsilon \boldsymbol{E}=\boldsymbol{E}+4 \pi \boldsymbol{P}$ ) on the other hand, leads to the Clausius-Mossotti relation for spherical particles [39]:

\section{Clausius-Mossotti relation}

$$
\alpha=\frac{3 V}{4 \pi} \frac{\varepsilon_{r}-1}{\varepsilon_{r}+2}
$$

where $V$ is the volume and $\varepsilon_{r}$ is the effective dielectric function. The expressions for the scattering and absorption cross section then simply follow as [90]

\section{Cross sections within the quasistatic approximation}

$$
C_{\mathrm{sca}}=\frac{8 \pi}{3} k^{4}|\alpha|^{2}, \quad C_{\mathrm{abs}}=4 \pi k \Im m\{\alpha\}, \quad C_{\mathrm{ext}}=C_{\mathrm{sca}}+C_{\mathrm{abs}} .
$$




\subsubsection{Mie theory}

The solution of Maxwell's equations for spherical particles (or infinitely long cylinders) is named after the physicist Gustav $\mathrm{Mie}^{2}$ and the expansion for elliptical particles became known as Gans or Mie-Gans theory ${ }^{3}$, see Sec. 4.1.3. An interesting overview about Mie's theory annotated with historical remarks can be found in [91], which was written in 2008 at the occasion of the centenary anniversary of Mie's original publication [92].

A rigorous derivation of Mie's formal solution can be found in [90,92-94] for example, we will only present the basic steps here. We are going to calculate the time-harmonic electromagnetic field of a sphere of arbitrary size embedded in a linear, isotropic, homogeneous medium. Because of the spherical symmetry of the problem, the use of spherical harmonic functions and a multipole extension of the fields is clearly an advantage. As shown in App. A.6, we can introduce vector harmonics $\mathcal{M}$ and $\mathcal{N}$ that satisfy the wave Eq. (3.16) and have all the required properties of an electromagnetic field. The scalar function $\psi$ is called the generating function for these vector harmonics, see Eq. (A.43). With $\psi$ the problem of finding solutions for the electromagnetic fields reduces to the comparatively simpler problem of finding solutions to the scalar wave equation, see Eq. (A.47). The symmetry of the investigated problem dictates the choice of generating functions, i.e. in our case $\psi$ is a function of spherical coordinates:

\section{Scalar wave equation in spherical coordinates}

$$
\left[\frac{1}{r^{2}} \frac{\partial}{\partial r}\left(r^{2} \frac{\partial}{\partial r}\right)+\frac{1}{r^{2} \sin \theta} \frac{\partial}{\partial \theta}\left(\sin \theta \frac{\partial}{\partial \theta}\right)+\frac{1}{r^{2} \sin ^{2} \theta} \frac{\partial^{2}}{\partial \varphi^{2}}\right] \psi=0
$$

This equation can be solved by the usual product ansatz for $\psi(r, \theta, \varphi)$, as discussed App. A.5 for example. This leads to three decoupled differential equations and after

\footnotetext{
${ }^{2}$ Born $29^{\text {th }}$ September 1868 in Rostock $; ~ \uparrow 13^{\text {th }}$ February 1957 in Freiburg im Breisgau.

${ }^{3}$ At this point usually a little pedantism sets in and we should not speak of it as a theory but rather call it Mie oder Mie-Gans solution, since it is just a result of Maxwell's equations under certain circumstances.
} 
some algebra [93] we can construct the solution of (4.3) as a linear combination of even and odd generating functions [90]:

$$
\psi_{l m}= \begin{cases}\cos (m \varphi) P_{l}^{m}(\cos \theta) z_{l}(k r), & \text { even solution } \\ \sin (m \varphi) P_{l}^{m}(\cos \theta) z_{l}(k r), & \text { odd solution }\end{cases}
$$

where $P_{l}^{m}(\cos \theta)$ are the associated Legendre functions of the first kind of degree $l$ and order $m$. The symbol $z_{l}$ is a substitute for any of the four spherical Bessel functions $j_{l}, y_{l}, h_{l}^{(1)}$, or $h_{l}^{(2)}$. Because of the completeness of each individual function on the right hand side of Eq. (4.4), $\psi_{l m}$ may serve as a basis and any quantity that fulfills Eq. (4.3) may be expanded as an infinite series in these generating functions. But since we are interested in solutions of the field equations, it is more convenient to go one step backwards and use the vector spherical harmonics as basis:

$$
\mathcal{M}_{l m}=\nabla \times\left(\boldsymbol{r} \psi_{l m}\right), \quad \mathcal{N}_{l m}=\frac{1}{n k}\left(\nabla \times \mathcal{M}_{l m}\right)
$$

If $u$ and $v$ are two solutions of Eq. (4.3), we can derive Maxwell's electromagnetic fields in terms of $\mathcal{M}_{u}, \mathcal{N}_{u}, \mathcal{M}_{v}$, and $\mathcal{N}_{v}$ (see [93] for the proof)

$$
\begin{aligned}
\boldsymbol{E} & =\boldsymbol{\mathcal { M }}_{v}+i \mathcal{N}_{u} \\
\boldsymbol{H} & =n\left(-\boldsymbol{M}_{u}+i \mathcal{N}_{v}\right)
\end{aligned}
$$

Before we are able to derive Mie's solution for the scattering of a plane wave, we still have to express the incident plane wave in the same basis functions. This derivation can again be found in many textbooks and the interested reader may once again be referred to the excellent book of Bohren and Huffman [93] - and thereby acquire virtue through suffering, in the words of Bohren and Huffman [93]: ". . this is undoubtedly the result of the unwillingness of a plane wave to wear a guise in which it feels uncomfortable; expanding a plane wave in spherical wave functions is somewhat like trying to force a square peg into a round hole." 
It can be proven, that the following two choices of $u$ and $v$ together with Eq. (4.6) generate an adequate expression for the incident plane wave:

$$
\begin{aligned}
& u=e^{-i \omega t} \cos (\varphi) \sum_{l=1}^{\infty}(-i)^{l} \frac{2 l+1}{l(l+1)} P_{l}^{1}(\cos \theta) j_{l}(k r), \\
& v=e^{-i \omega t} \sin (\varphi) \sum_{l=1}^{\infty}(-i)^{l} \frac{2 l+1}{l(l+1)} P_{l}^{1}(\cos \theta) j_{l}(k r),
\end{aligned}
$$

The field outside the sphere is then given by a superposition of the incident plane wave plus the scattered wave. If we exploit Maxwell's boundary conditions and the conditions to be satisfied at infinity we find the following general expressions:

\section{Outside Mie solution, scattered wave}

$$
\begin{aligned}
& u=e^{-i \omega t} \cos (\varphi) \sum_{l=1}^{\infty} a_{l}(i)^{l} \frac{2 l+1}{l(l+1)} P_{l}^{1}(\cos \theta) h_{l}^{(2)}(k r), \\
& v=e^{-i \omega t} \sin (\varphi) \sum_{l=1}^{\infty} b_{l}(i)^{l} \frac{2 l+1}{l(l+1)} P_{l}^{1}(\cos \theta) h_{l}^{(2)}(k r) .
\end{aligned}
$$

Similarly, the field inside the sphere is given by

\section{Inside Mie solution}

$$
\begin{aligned}
& u=e^{-i \omega t} \cos (\varphi) \sum_{l=1}^{\infty} n c_{l}(-i)^{l} \frac{2 l+1}{l(l+1)} P_{l}^{1}(\cos \theta) j_{l}(n k r), \\
& v=e^{-i \omega t} \sin (\varphi) \sum_{l=1}^{\infty} n d_{l}(-i)^{l} \frac{2 l+1}{l(l+1)} P_{l}^{1}(\cos \theta) j_{l}(n k r) .
\end{aligned}
$$

The different appearance of the Bessel functions $h_{l}^{(2)}$ and $j_{l}$ is due to the asymptotic behavior of the scattered wave and the finite field at the origin, respectively. The 
expressions of the undetermined coefficients $a_{l}, b_{l}, c_{l}$, and $d_{l}$ follow once again from the boundary conditions. After some algebra (that once again can be found in [90, 93]) we derive the Mie coefficients for the outside field with the refractive index $n$ (remember $\mu=1$ ):

\section{Mie scattering coefficients}

$$
\begin{aligned}
a_{l} & =\frac{n^{2} \psi_{l}(n x) \psi_{l}^{\prime}(x)-\psi_{l}(x) \psi_{l}^{\prime}(n x)}{n^{2} \psi_{l}(n x) \xi_{l}^{\prime}(x)-\xi_{l}(x) \psi_{l}^{\prime}(n x)}, \\
b_{l} & =\frac{\psi_{l}(n x) \psi_{l}^{\prime}(x)-n \psi_{l}(x) \psi_{l}^{\prime}(n x)}{\psi_{l}(n x) \xi_{l}^{\prime}(x)-n \xi_{l}(x) \psi_{l}^{\prime}(n x)},
\end{aligned}
$$

where we have introduced the Riccati-Bessel functions

\section{Riccati-Bessel functions}

$$
\psi_{l}(\rho)=\rho j_{l}(\rho), \quad \xi_{l}(\rho)=\rho h_{l}^{(1)}(\rho) .
$$

Note that $a_{l}$ and $b_{l}$ vanish as $n$ approaches unity: When the particle disappears, so does the scattered field. Likewise we can derive the Mie coefficients for the inside field:

Mie coefficients for inside field

$$
\begin{aligned}
c_{l} & =\frac{\psi_{l}(x) \xi_{l}^{\prime}(x)-\xi_{l}(x) \psi_{l}^{\prime}(x)}{\psi_{l}(n x) \xi_{l}^{\prime}(x)-\xi_{l}(x) \psi_{l}^{\prime}(n x)}, \\
d_{l} & =\frac{n \psi_{l}(x) \xi_{l}^{\prime}(x)-n \xi_{l}(x) \psi_{l}^{\prime}(x)}{n^{2} \psi_{l}(n x) \xi_{l}^{\prime}(x)-\xi_{l}(x) \psi_{l}^{\prime}(n x)} .
\end{aligned}
$$




\section{Cross sections with Mie theory}

Once the Mie coefficients are determined, we can calculate the extinction, absorption and scattering cross sections or the electromagnetic fields inside and outside of the spherical particle.
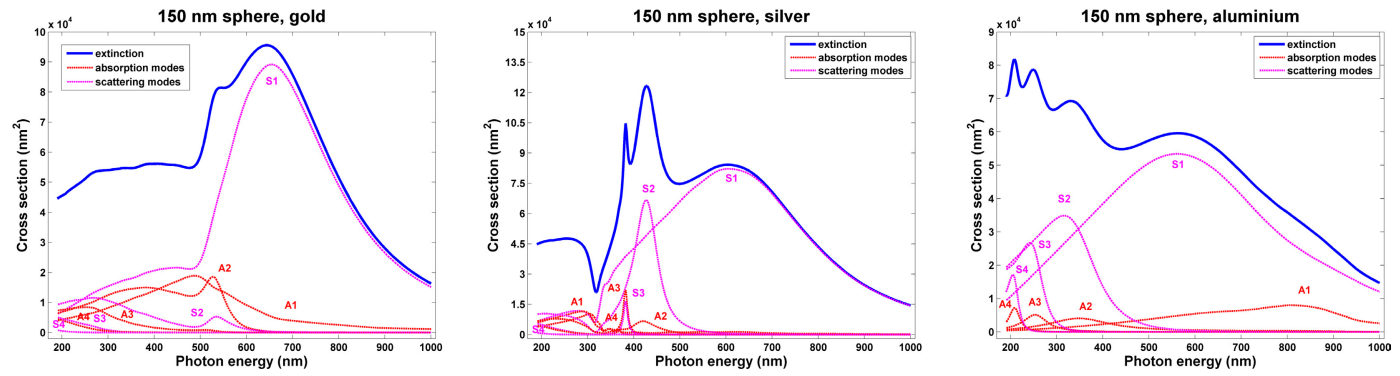

Figure 4.1.: Extinction cross sections of a sphere with $150 \mathrm{~nm}$ diameter made of different materials: (a) gold, (b) silver and (c) aluminum (measured values for $\varepsilon(\omega)$ from [41] and the dielectric function database of F. J. García de Abajo). The spectra can be decomposed into contributions from absorption $\left(A_{n}\right)$ and scattering modes $\left(S_{n}\right)$ with $n=1,2,3, \ldots$ for the dipole, quadrupole, octopole mode, $\ldots$ respectively (see also [91]). To simplify matters magnetic modes have been omitted here but also give important contributions.

A MATlaB ${ }^{\circledR}$ code example for Mie scattering can be found in App. C.2 (a dielectric table containing the photon energy in $\mathrm{eV}$ and the corresponding real and imaginary part of the refractive index has to be provided, cf. [41]). An analogue example for Mathematica $^{\mathrm{TM}}$ can be found in [42].

\subsubsection{Mie-Gans solution}

An additional analytical solution for elliptical, spheroidal particles is also possible and is called Mie-Gans solution [90,93]. Scattering characteristics for oblate and prolate spheroidal particles can be calculated, as long as a quasistatic approximation is adopted.

The three main values $\alpha_{1}, \alpha_{2}, \alpha_{3}$ of the polarizability tensor can be computed with [90] 


\section{Polarizations for Mie-Gans solution}

$$
\alpha_{i}=\frac{V}{4 \pi}\left(L_{i}+\frac{1}{\varepsilon_{r}-1}\right)^{-1} .
$$

The geometrical factors $L_{i}$ are related to the particle shape and always fulfill the sum rule $L_{1}+L_{2}+L_{3}=1$. Thus for a sphere we get $L_{i}=1 / 3$ for all $i$ and we immediately recover the Clausius-Mossotti relation (4.1).
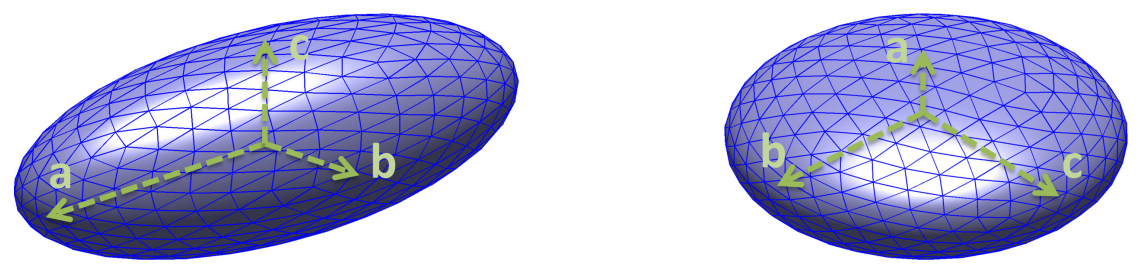

Figure 4.2.: Prolate (left, $a>b=c$ ) and oblate (right, $a<b=c$ ) ellipsoids.

For an arbitrary ratio of the particle's semiaxis $a, b$, and $c$ the factor $L_{i}$ can be calculated from [90]

$$
L_{1}=\int_{0}^{\infty} \frac{a b c d s}{2\left(s+a^{2}\right)^{3 / 2}\left(s+b^{2}\right)^{1 / 2}\left(s+c^{2}\right)^{1 / 2}}
$$

with cyclical changes for $L_{2}$ and $L_{3}$. For the special case of spheroidal particles ( $b=c$, see Fig. 4.2), we get the solutions [90]

$$
\begin{array}{lll}
\text { prolate }(a>b): & L_{1}=\frac{1-e^{2}}{e^{2}}\left(-1+\frac{1}{2 e} \ln \frac{1+e}{1-e}\right), & e=1-\left(\frac{b^{2}}{a^{2}}\right), \\
\text { oblate }(a<b): & L_{1}=\frac{1+f^{2}}{f^{2}}\left(1-\frac{1}{f} \arctan f\right), & f=\left(\frac{b^{2}}{a^{2}}\right)-1 .
\end{array}
$$

The corresponding cross sections again follow from Eq. (4.2), with $\alpha=\sum_{i} \alpha_{i} \hat{e}_{i}$, where $\hat{e}$ is the light polarization. 


\subsection{Discrete Dipole Approximation}

The Discrete Dipole Approximation (DDA) is a numerical technique for computing electromagnetic scattering and absorption by targets of arbitrary shape [95], where the continuum target is approximated by a finite point array. In response to the local electric field each point acquires a dipole moment and the scattering problem can then be solved in a self-consistent way. Thus, in principle this method is completely flexible regarding the geometry of the target, the only limitation is given by the need to use an interdipole separation that is small compared to any structural lengths in the target and to the wavelength $\lambda[96]$. The theoretical basis for the DDA is summarized in [97].

\section{Additional remark}

Nature provides the physical inspiration for the DDA: In 1909 Lorentz showed [98] that the dielectric properties of a substance could be directly related to the polarizabilities of the individual atoms of which it was composed, with a particularly simple and exact relationship, the Clausius-Mossotti relation (4.1), when the atoms are located on a cubic lattice. We may expect that, just as a continuum representation of a solid is appropriate on length scales that are large compared with the interatomic spacing, an array of polarizable points can accurately approximate the response of a continuum target on length scales that are large compared with the interdipole separation.

For a finite array of point dipoles the scattering problem may be solved exactly, so the only approximation that is present in the DDA is the replacement of the continuum target by an array of $\mathrm{N}$-point dipoles.

This annotation is adopted from [95].

The basic idea of the DDA was already known in 1964, but it was limited to structures that were small compared to the wavelength. This limitation disappeared, when Purcell and Pennypacker introduced the DDA to study interstellar dust grains in 1973 [99]. Fortunately the method is not restricted to astrophysics and Draine and 
Flatau published a free ForTRAN software package (DDSCAT, current version 7.1) that can be applied to plasmonic scattering problems.

By following [97] very closely, we will shortly show that with the DDA the problem of electromagnetic scattering of an incident light wave can then be cast to the following simple matrix equation

\section{Discrete Dipole Approximation}

$$
\tilde{\boldsymbol{A}} \tilde{\boldsymbol{P}}=\tilde{\boldsymbol{E}}_{\mathrm{inc}},
$$

where $\tilde{\boldsymbol{E}}_{\text {inc }}$ is a $3 N$-dimensional (complex) vector of the incident electric field at the $N$ lattice sites, $\tilde{\boldsymbol{P}}$ is a $3 N$-dimensional (complex) vector of the (unknown) dipole polarizations, and $\tilde{\boldsymbol{A}}$ is a $3 N \times 3 N$ complex matrix.

Let us assume a point lattice with $N$ occupied sites and an index $j=1, \ldots, N$ running over these elements. Each dipole $\boldsymbol{j}$ is characterized by a polarizability tensor $\boldsymbol{\alpha}_{j}$, which is diagonal with equal components if the material is isotropic (i.e., $\boldsymbol{\alpha}_{j}$ may be treated as a scalar quantity in this case). We will restrict our attention to the case where all individual dipole polarizability tensors can be simultaneously diagonalized, although it is straightforward to generalize the problem to non diagonal tensors (see [97] for more details). It is nontrivial to choose an adequate $\boldsymbol{\alpha}_{j}$ for the individual dipoles - Purcell and Pennypacker for example used the Clausius-Mossotti relation (4.1) to obtain an estimate for the polarizability. This assumption is exact in the zero-frequency limit, but it fails at finite $\omega$ [97].

Let $\boldsymbol{P}_{j}$ be the instantaneous complex dipole moment of dipole $\boldsymbol{j}$, and $\boldsymbol{E}_{\text {ext }, j}$ the instantaneous complex electric field at position $j$ due to the incident radiation plus the other $N-1$ oscillating dipoles. Then we get

$$
\boldsymbol{P}_{j}=\boldsymbol{\alpha}_{j} \boldsymbol{E}_{\mathrm{ext}, j}
$$


As noted in [99], this expression can now be recast as $N$ simultaneous vector equations of the form

$$
\boldsymbol{P}_{j}=\boldsymbol{\alpha}_{j}\left(\boldsymbol{E}_{\mathrm{inc}, j}-\sum_{k \neq j} \boldsymbol{A}_{j k} \boldsymbol{P}_{k}\right)
$$

where $\boldsymbol{E}_{\text {inc,j }}$ corresponds to the electric field of the incident plane wave at position $j$,

$$
\boldsymbol{E}_{\mathrm{inc}, j}=\boldsymbol{E}_{0} \exp \left(i \boldsymbol{k} \cdot \boldsymbol{r}_{j}-i \omega t\right)
$$

and $-\boldsymbol{A}_{j k} \boldsymbol{P}_{k}$ is the contribution to the electric field at position $j$ due to the dipole at position $k$. The matrices $\boldsymbol{A}_{j k}$ are defined for $j \neq k$ through

$$
\boldsymbol{A}_{j k} \boldsymbol{P}_{k}=\frac{e^{i k r_{j k}}}{r_{j k}^{3}}\left\{k^{2} \boldsymbol{r}_{j k} \times\left(\boldsymbol{r}_{j k} \times \boldsymbol{P}_{k}\right)+\frac{1-i k r_{j k}}{r_{j k}^{2}} \times\left[r_{j k}^{2} \boldsymbol{P}_{k}-3 \boldsymbol{r}_{j k}\left(\boldsymbol{r}_{j k} \cdot \boldsymbol{P}_{k}\right)\right]\right\}
$$

where $\boldsymbol{r}_{j k} \equiv \boldsymbol{r}_{j}-\boldsymbol{r}_{k}$ and $r_{j k}=\left|\boldsymbol{r}_{j k}\right|$. By defining the matrix elements for $j=k$ as $\boldsymbol{A}_{j j}=\boldsymbol{\alpha}_{j}^{-1}$, the scattering problem can be compactly formulated as

$$
\sum_{k=1}^{N} \boldsymbol{A}_{j k} \boldsymbol{P}_{k}=\boldsymbol{E}_{\mathrm{inc}, j} \quad(j=1, \ldots, N)
$$

If we now introduce the two $3 N$-dimensional vectors $\tilde{\boldsymbol{P}}=\left(\boldsymbol{P}_{1}, \boldsymbol{P}_{2}, \ldots, \boldsymbol{P}_{N}\right)$ and $\tilde{\boldsymbol{E}}_{\text {inc }}=\left(\boldsymbol{E}_{\text {inc }, 1}, \boldsymbol{E}_{\text {inc }, 2}, \ldots, \boldsymbol{E}_{\text {inc }, N}\right)$ and the $3 N \times 3 N$ symmetric matrix $\tilde{\boldsymbol{A}}$, we recover the single matrix Eq. (4.15) from the beginning of this section:

$$
\tilde{\boldsymbol{A}} \tilde{\boldsymbol{P}}=\tilde{\boldsymbol{E}}_{\text {inc }} .
$$

The most simple (and brute) method to solve this system for the unknown vector $\tilde{\boldsymbol{P}}$ is a direct inversion of $\tilde{\boldsymbol{A}}$. But since $3 N$ is a large number (For a typical nanoparticle the number of dipoles is of the order of $10^{4}$ to $10^{5}$ !), such an direct method is quite impractical. Many different techniques for solving such equation systems are available and DDSCAT uses an iterative method [96]. It begins with a guess (typically $\tilde{\boldsymbol{P}}=\mathbf{0}$ ) 
for the unknown polarization vector, and then iteratively improves the estimate for $\tilde{\boldsymbol{P}}$ until Eq. (4.15) is solved to some error criterion (usually the error tolerance is user-defined and smaller than $10^{-5}$, see [96]). Under some circumstances there may be problems with the convergence of this iterative method (see for example [99]), so in general convergence tests are necessary to choose the right grid size.

\subsubsection{Cross sections with DDA}

Once Eq. (4.15) has been solved, the extinction $C_{\text {ext }}$, absorption $C_{\text {abs }}$, and scattering $C_{\text {sca }}$ cross sections can be computed from the optical theorem. The results can again be found in [97] and are given by

\section{Cross sections with DDA}

$$
\begin{aligned}
C_{\mathrm{ext}} & =\frac{4 \pi k}{\left|\boldsymbol{E}_{\mathrm{inc}}\right|^{2}} \sum_{j=1}^{N} \Im m\left(\boldsymbol{E}_{\mathrm{inc}, j}^{*} \cdot \boldsymbol{P}_{j}\right), \\
C_{\mathrm{abs}} & =\frac{4 \pi k}{\left|\boldsymbol{E}_{\mathrm{inc}}\right|^{2}} \sum_{j=1}^{N}\left\{\Im m\left[\boldsymbol{P}_{j} \cdot\left(\boldsymbol{\alpha}_{j}^{-1}\right)^{*} \boldsymbol{P}_{j}^{*}\right]-\frac{2}{3} k^{3} \boldsymbol{P}_{j} \cdot \boldsymbol{P}_{j}^{*}\right\}, \\
C_{\mathrm{sca}} & \equiv C_{\mathrm{ext}}-C_{\mathrm{abs}}
\end{aligned}
$$

with the wave number $k=2 \pi / \lambda$. When absorption is dominant, it may become difficult to calculate $C_{\text {sca }}$ with the above subtraction, because then $C_{\text {ext }}$ and $C_{\text {abs }}$ have to be computed with high accuracy. It is also possible to compute the scattering cross section directly by calculating the power radiated by the array of oscillating dipoles [97]:

$$
C_{\text {sca }}=\frac{k^{4}}{\left|\boldsymbol{E}_{i n c}\right|^{2}} \int d \Omega\left|\sum_{j=1}^{N}\left[\boldsymbol{P}_{j}-\hat{\boldsymbol{n}}\left(\hat{\boldsymbol{n}} \cdot \boldsymbol{P}_{j}\right)\right] e^{-i k \hat{\boldsymbol{n}} \cdot \boldsymbol{r}_{j}}\right|^{2},
$$

where $\hat{\boldsymbol{n}}$ is an unit vector in the direction of the scattering, and $d \Omega$ is the element of solid angle. 


\subsection{Boundary Element Method}

The Boundary Element Method (BEM) has emerged as a powerful numerical technique for solving a wide variety of computational engineering and science problems ${ }^{4}$. The method has remote deep roots in the history of mechanics ${ }^{5}$ and its mathematical foundations include the theorems of Gauss, Green and Stokes - they allow the basic reduction from volume differential equations to boundary integral equations [100] as we have already seen in Chap. 3 .

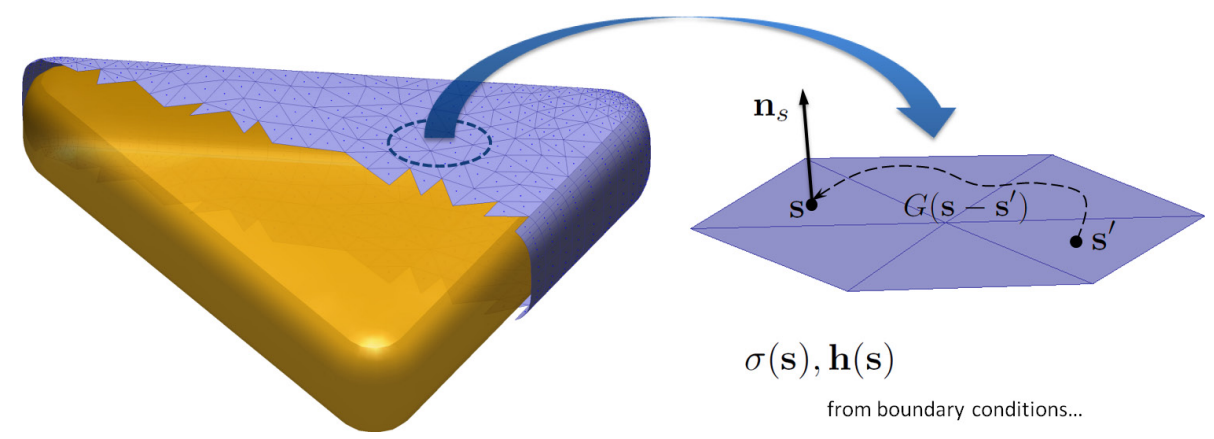

Figure 4.3.: After discretizing the particle surface the corresponding continuous surface charges and currents are approximated as points in the centroids of the small surface elements. The Green function connects these different points with each other, see Eq. (3.38).

The first step in developing the BEM is based upon transforming the considered set of differential equation [viz. Maxwell's equations, see Eq. (3.19)] into integral equations [we did this in Eq. (3.24) and Eq. (3.40)]. The new integral expressions are valid everywhere - inside and outside the domain as well as on its boundary. Now the structure of the considered problem comes into play: Usually we can solve the equations inside and outside the domain, the only non-trivial part comes from the boundary (note that this approach is based upon a mathematically rigorous definition of the integrals as limits to the boundary [100]). As mentioned in Sec. 3.3.1, the BEM is based on an approximation of a continuous surface to a discrete number of points located at the centroids of small surface elements, see Fig. 4.3 - this is the so called

\footnotetext{
${ }^{4}$ Examples of application cover the fields of elasticity, geomechanics, structural mechanics, electromagnetics, acoustics, hydraulics, biomechanics, and much more.

${ }^{5} \mathrm{~A}$ short overview about the historical development of the BEM can be found in [100] for example.
} 
collocation method, in contrast to the more complex Galerkin approach [100], for example, where a linear interpolation within the surface elements is used.

Once the surface charges and currents have been derived as discussed in Chap. 3 (see Eq. (3.31) and Eq. (3.44)), we can determine the electromagnetic fields (see Fig. 4.4) and simply compute the scattering, absorption and extinction cross sections from the Poynting vector $[66,88]$ :

\section{Cross sections for retarded simulation}

$$
\begin{aligned}
& C_{\mathrm{sca}}=n_{b} \oint_{\partial \Omega} \Re e\left\{n\left(\boldsymbol{E} \times \boldsymbol{B}^{*}\right)\right\} d a, \\
& C_{\mathrm{ext}}=-\frac{1}{n_{b}} \oint_{\partial \Omega} \Re e\left\{n\left(\boldsymbol{E} \times \boldsymbol{B}_{\mathrm{inc}}^{*}+\boldsymbol{E}_{\mathrm{inc}}^{*} \times \boldsymbol{B}\right)\right\} d a, \\
& C_{\mathrm{abs}}=P_{\mathrm{ext}}-P_{\mathrm{sca}} .
\end{aligned}
$$
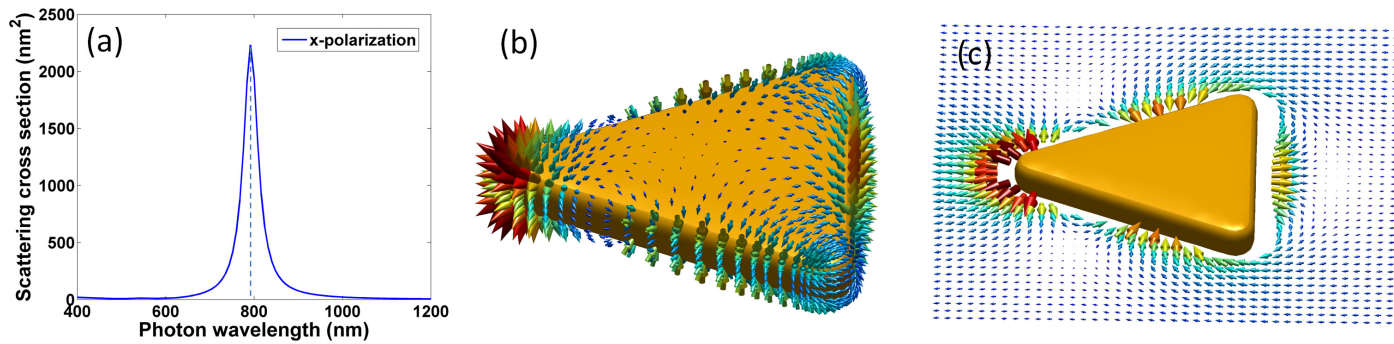

Figure 4.4.: (a) Scattering cross section of a gold nanotriangle $\left(55 \times 50 \times 8 \mathrm{~nm}, n_{b}\right.$ $=1.34$ ). The panels (b) and (c) show the electric field at the resonance energy of $792 \mathrm{~nm}$ at the particle surface and on the outside, respectively. (Also see Fig. 3.7.)

Related to this thesis we have developed a MATLAB ${ }^{\circledR}$ toolbox called MNPBEM for the simulation of metallic nanoparticles based on the BEM. Further details on this toolbox can be found in [84] and [88]. 


\subsection{Other methods}

There exists a whole bunch of other methods for the calculation of light scattering and absorption of plasmonic structures. The majority of them is based on a discretization of space (and time). For example with the Finite Difference Time Domain (FDTD) approach the hole space containing the particle together with the time parameter has to be discretized and Maxwell's equations are solved in their differential form. Another technique is based on the dyadic Green tensor, which mediates the response between small volume elements of the scatterer. Other approaches are the multiple multipole method [4], the Method of Moments (MoM), or the multiple scattering method [23]. 


\subsection{Comparison between different approaches}

In this section we will compare some of the different methods discussed before and highlight the pros and cons of the individual approaches. There exist several papers in the literature where a more extensive comparison is performed, for example see $[23,60]$ or [101-103] and references therein.

\subsubsection{Accuracy}

It is possible to generate very accurate results with all different methods, as long as the discretization is chosen appropriately. In Fig. 4.5 the simulation results of BEM and DDSCAT are compared to the analytical Mie solution.

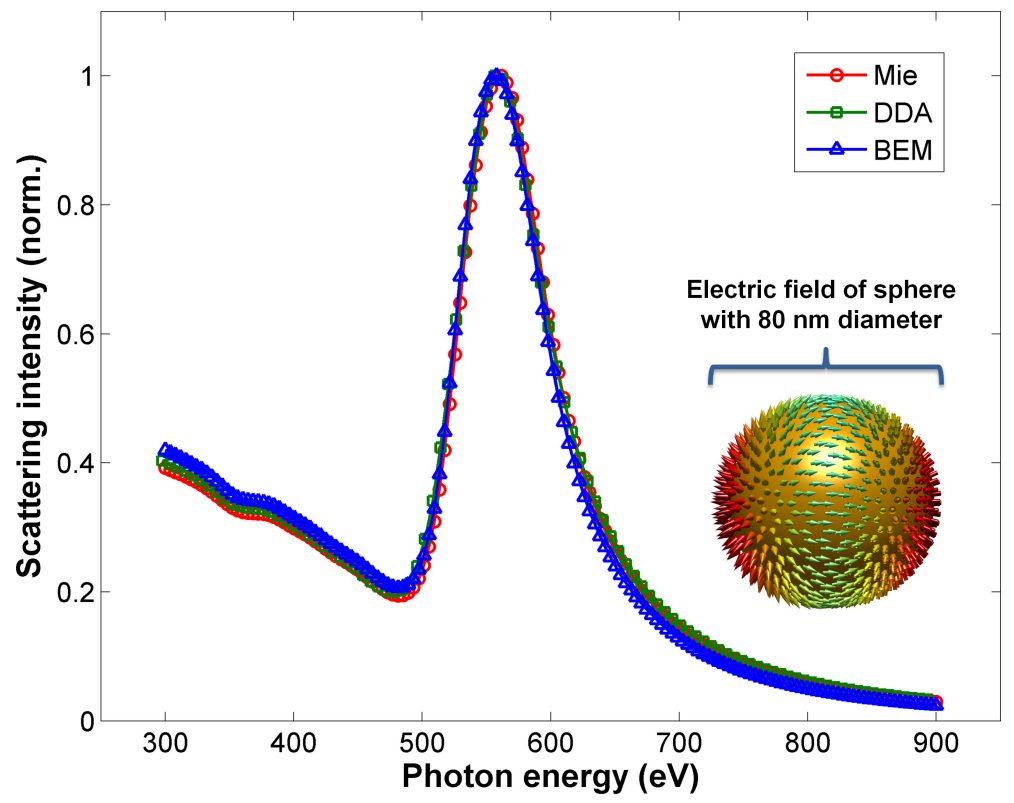

Figure 4.5.: Normalized scattering cross section of a gold sphere with $80 \mathrm{~nm}$ diameter in water with $\varepsilon_{b}=1.34^{2}$, calculated for plane wave illumination with $\mathrm{x}$-polarization with different methods. Whereas the BEM result followed almost instantaneous, the DDA simulation took more than one day, see Tab. 4.2.

The agreement between BEM and DDA simulations is very good, also if we change to other particle shapes, see Fig. 4.6. 

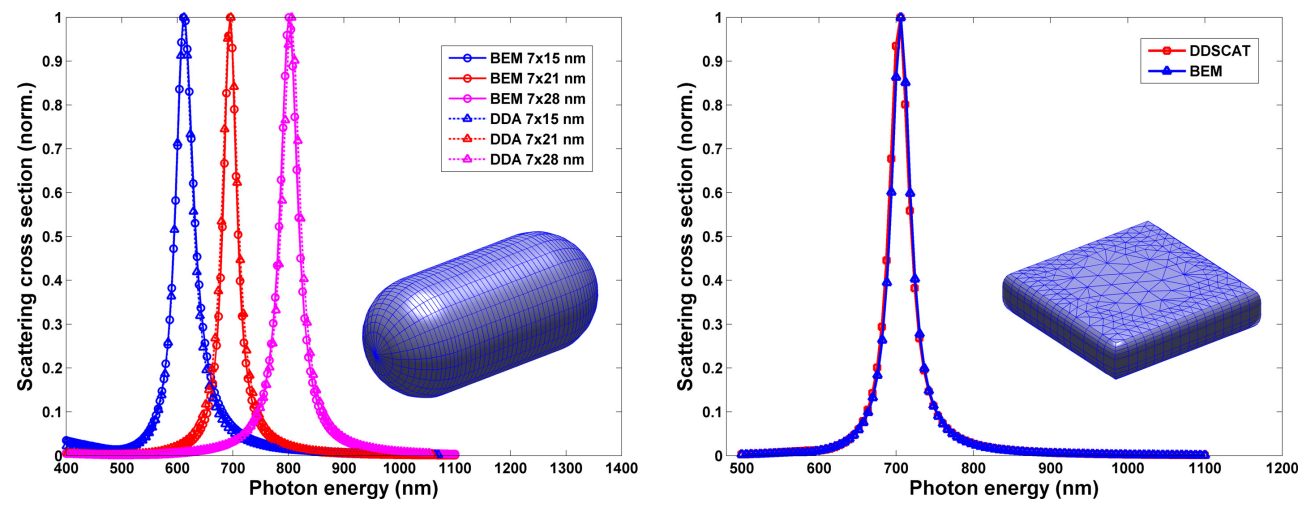

Figure 4.6.: Comparison of the normalized scattering cross section calculated with $\mathrm{BEM}$ and DDSCAT for gold rods with changing aspect ratio (left) and a $15 \mathrm{x} 15 \mathrm{x}$ $8 \mathrm{~nm}$ gold cube (right), $\varepsilon_{b}=1.34^{2}$.

\subsubsection{Performance}

A performance comparison for the different methods is a challenging task since all of them have their own assets and drawbacks. In this subsection we will only show scattering results for different particle geometries which we obtained from BEM and DDA simulations. For the DDA simulation we used the previously discussed DDSCAT program. The BEM simulations were carried out with our MNPBEM toolbox.

In Tab. 4.1 a computing time comparison for the quasistatic and the full retarded solution of Maxwell's equations as discussed in Chap. 3 is shown. Tab. 4.2 is adopted from [60], for details see table caption.

\begin{tabular}{ccccc}
\hline No. of vertices & Nr. of faces & quasistatic & $\begin{array}{c}\text { eigenmode } \\
\text { expansion }\end{array}$ & $\begin{array}{c}\text { full retarded } \\
\text { solution }\end{array}$ \\
\hline 144 & 284 & $2.26 \mathrm{~s}$ & $0.23 \mathrm{~s}$ & $8.81 \mathrm{~s}$ \\
256 & 508 & $4.83 \mathrm{~s}$ & $0.43 \mathrm{~s}$ & $32.85 \mathrm{~s}$ \\
400 & 796 & $16.05 \mathrm{~s}$ & $0.94 \mathrm{~s}$ & $103.96 \mathrm{~s}$ \\
676 & 1348 & $71.82 \mathrm{~s}$ & $2.82 \mathrm{~s}$ & $446.38 \mathrm{~s}$ \\
\hline
\end{tabular}

Table 4.1.: CPU time elapsed for a $10 \mathrm{~nm}$ sphere with changing discretization for different kinds of BEM simulations, see [84]. The calculations were performed on a standard office PC running on Windows XP with MATLAB ${ }^{\circledR}$ R2010b 32-bit. 


\begin{tabular}{|c|c|c|c|}
\hline & BEM & $\mathrm{DDA}$ & FDTD \\
\hline $\begin{array}{l}\text { Computational } \\
\text { demand }\end{array}$ & $V^{2} N_{\omega}$ & $V^{3} N_{\omega}$ & $V^{\prime} \omega / \Delta \omega$ \\
\hline Storage demand & $V^{4 / 3}$ & $V^{2}$ & $V^{\prime}$ \\
\hline $\begin{array}{l}\text { Average CPU time } \\
\text { for } 80 \mathrm{~nm} \text { sphere }\end{array}$ & $1 \mathrm{~min}$ & $26 \mathrm{~h}(\sim 2$ days $)$ & not tested yet \\
\hline Advantages & $\begin{array}{l}\text { low computational } \\
\text { and storage demand, } \\
\text { only surface is } \\
\text { discretized }\end{array}$ & $\begin{array}{c}\text { simple } \\
\text { parametrization, only } \\
\text { particle volume is } \\
\text { discretized }\end{array}$ & $\begin{array}{c}\text { simple } \\
\text { implementation, full } \\
\text { spectrum in single } \\
\text { run }\end{array}$ \\
\hline Disadvantages & $\begin{array}{c}\text { complex } \\
\text { parametrization }\end{array}$ & $\begin{array}{c}\text { time consuming, } \\
\text { particle size } a \text { and } \varepsilon \\
\text { limited to }|a \sqrt{\varepsilon}| / \lambda<5\end{array}$ & $\begin{array}{l}\text { time consuming, } \\
\text { difficult to apply to } \\
\text { arb. } \varepsilon(\omega) \text {, parametr. } \\
\text { of outside volume } \\
\text { and ABCs. }\end{array}$ \\
\hline
\end{tabular}

Table 4.2.: Critical comparison of the performance of BEM, DDA, and FDTD adopted from [60], where we have added a CPU time comparison. $N_{\omega}$ is the number of calculated frequencies and $V$ is the particle volume measured in units of the cube of the skin depth [see Eq. (2.9), $\zeta \approx 15 \mathrm{~nm}$ in the visible near-infrared (see App. A.2 for the frequency range of the visible spectrum)]. $V^{\prime}$ is the discretized volume for the FDTD simulation and it extends considerably outside the particle in order to account for light propagation in the surrounding medium [also absorbing boundary conditions (ABCs) have to be applied]. In this table for the BEM and DDA approach a direct inversion of the secular linear equations is assumed (DDSCAT uses conjugate gradient algorithms to solve the corresponding equations in an iterative approach - three different algorithms can be chosen in the current version of DDSCAT [96]. DDA scales as $V \log V N_{\omega}$ when using the iterative method, whereas BEM scales as $V^{4 / 3} N_{\omega}$ in this case, see [60]). The factor $\omega / \Delta \omega$ in FDTD is proportional to the number of time steps needed to describe a component of frequency $\omega$ with frequency resolution $\Delta \omega$. For the comparison of a typical average computing time the optical spectrum of a gold sphere with a diameter of $80 \mathrm{~nm}$ and $N_{\omega}=150$ has been calculated (particle symmetry has not been exploited). The DDA simulation ran on 32 nodes of a Sun Fire V20z cluster with $2 \times$ AMD Opteron $2482(2 \mathrm{GHz})$ processors each (the number in brackets corresponds to the simulation time on only 4 nodes to allow a better comparison), whereas the BEM simulation was started on a transtec CALLEO 431L server with four AMD six-core Opteron F 2431 (2.4 GHz) processors and 64 GB DDR2-667 memory. On a standard PC the same BEM simulation takes about 2 minutes, see Tab. 4.1. 


\subsubsection{Limits and inaccuracies}

The BEM approach is suited for homogeneous and isotropic dielectric environments, where the embedded bodies are separated by sharp boundaries. Besides the computational limitations (for an overview of the MATLAB ${ }^{\circledR}$ memory limitations for different operating systems consult App. C.1), there are some other points, that are responsible for inaccuracies or difficult (or impossible) to implement. Nevertheless the BEM works remarkably well and the agreement with experimental data is striking. Here is a short list of possible problems that are inherent to all discussed simulation methods (fortunately they mostly become important only if the nanoparticles are very small):

- The sharp and abrupt two dimensional Maxwell boundary conditions become questionable for very small particles because of the spill-out effect [91]: The surfaces boundary changes to three dimensional inhomogeneous interface.

- The surface and interface gets charged because of the different chemical potential of the particle and dielectric background (chemical interface damping [104]).

- The dielectric function of a nanoparticle is different from the dielectric function of bulk material.

- As already mentioned before, the dielectric function $\varepsilon(\omega)$ is not homogeneous, it is a local spatial dependent function $\varepsilon\left(\boldsymbol{r}, \boldsymbol{r}^{\prime}, \omega\right)$ and changes continuously while approaching the surface or defects in the material [91].

- The idea of a surface charge density or a surface current density is an idealization of macroscopic electromagnetism. In reality the charge or current is confined to the immediate neighborhood of the surface [66]. (Nevertheless this idealization works quite well and is an essential part of classical field theory.) 



\section{Imaging of surface plasmons}

Maxwell's equations are about 150 years old, so why (without any intention to be blasphemous) can we still do interesting physics with them? The answer lies in the interplay with sources: Electromagnetic interactions down at the nanoscale open up the fascinating field of nanooptics, interactions with quantum objects allow us to enter the prosperous world of quantum optics.

\begin{tabular}{lccc}
\hline coherent (classical) optics & $L$ & $(\gg)>$ & $\lambda$ \\
diffraction grating & $L$ & $>$ & $\lambda$ \\
photonic crystals & $L$ & $(\ll)<$ & $\lambda$ \\
metamaterials & $L$ & $\gg$ & $\lambda$ \\
\hline
\end{tabular}

Table 5.1.: Characteristic length scales $L$ of different parts of optics compared to the wavelength $\lambda$.

\subsection{Principles of near-field optics}

In free space, the propagation of light is determined by the dispersion relation $\hbar \omega=c p=c \hbar k$, which connects the wave vector $k=\sqrt{k_{x}^{2}+k_{y}^{2}+k_{z}^{2}}=2 \pi / \lambda$ of a photon (for a real lens the wavelength $\lambda$ must be corrected by the numerical aperture NA) with its angular frequency $\omega[4]$. Heisenberg's uncertainty principle now already predicts a fundamental resolution limit for optical microscopes: The product of the uncertainty of the momentum of a microscopic particle $\Delta k_{x}$ and the uncertainty in the spatial position $\Delta x$ in the same direction cannot become smaller than $\hbar / 2$ : 


$$
\hbar \Delta k_{x} \cdot \Delta x \geq \frac{\hbar}{2}
$$

Since the maximal possible value of $k_{x}$ is the total length of the free-space wave vector $2 \pi / \lambda$, we can rewrite the uncertainty equation to

$$
\Delta x \geq \frac{1}{2 \Delta k_{x}}=\frac{\lambda}{4 \pi}
$$

This states, that the accomplishable spatial confinement for photons is inversely proportional to the spread ${ }^{1}$ in the magnitude of the associated wave vector component $\Delta k_{x}$.

The expression (5.2) is very similar to the diffraction-limited resolution derived by Lord Rayleigh [105] or Ernst Abbe $^{2}$ [106] in the late 19th century:

$$
\Delta x=0.6098 \frac{\lambda}{\mathrm{NA}}
$$

We see that there is some arbitrariness in the definition of a resolution limit: We started this discussion with the uncertainty principle of Quantum mechanics, Lord Rayleigh investigated a grating spectrometer [4], while Abbe based his formulation on the distinguishability of the image pattern of two point dipoles. A simple Fourier transformation from position to momentum space also leads us to a similar result:

$$
\Delta k_{x} \cdot \Delta x \geq 1, \quad \Longrightarrow \quad \Delta x \geq \frac{\lambda}{\pi \mathrm{NA}} \approx 0.3183 \frac{\lambda}{\mathrm{NA}},
$$

see [4] for more details. This last expression is almost two times less pessimistic than Eq. (5.3), but it seems that we cannot get a better resolution than approximately half of the wavelength. Does this predict that there is no reasonable chance to investigate objects on the nanoscale without violating fundamental laws of nature? Fortunately the answer is no, there are several ways to elude this limitation. On one

\footnotetext{
${ }^{1}$ Such a spread in wave vector components occurs for instance in a light field that converges toward a focus, e.g. behind a lens [4].

${ }^{2}$ Born $23^{\text {th }}$ January 1840 in Eisenach; $\dagger 14^{\text {th }}$ January 1905 in Jena.
} 
hand we can apply some tricks based on the exploitation of distinctive features of the investigated objects. For example we can increase the resolution trough selective excitation of two close-by molecules, if they are distinguishable in energy, orientation or something else.

But on the other hand if we look at Eq. (5.2) once again, we see that in theory there is no limit to optical resolution if the bandwidth of $\Delta k_{x}$ is arbitrarily large. In this sense if we include evanescent fields in optical microscopy we are able to increase the resolution limit and to investigate near fields and structures at the nanoscale. 


\subsection{How to picture a plasmon}

The previously discussed increase of the resolution limit is nicely realized in SNOM experiments for example, where we are able to map the photonic local density of states (LDOS). Another method that allows to directly picture surface plasmon polaritons of a metallic nanoparticle is EELS, see Chap. 11. In this approach a beam of electrons with high kinetic energy (of the order of $100 \mathrm{keV}$ ) passing by (or through) a metallic nanoparticle excites a plasmon and therefore looses a small fraction of its energy. By detecting this energy loss and rasterscanning the electron beam across the particle, we get a picture of the plasmon oscillation.

\subsubsection{Mapping the plasmonic LDOS}

The principal idea for SNOM is to bring a detector very close to surface of the investigated structure - as close that it can detect the evanescent fields. Such an detector can be realized by a fluorophore for example, since its spontaneous emission rate serves as a direct probe of the photonic LDOS at the fluorophore position. In Chap. 10 we exploit this capability for mapping the plasmonic modes of gold nanoparticles. We use combined regular arrays of identical gold and fluorophoredoped polymer nanoparticles with a slightly different grating constant. This setup allows th generate an optical Moiré pattern corresponding to a $200 \times$ magnified map of the plasmonic mode, which can be directly imaged with an optical microscope, see Fig. 5.1 and Fig. 10.3.

\subsubsection{Electron energy loss spectroscopy}

As mentioned above another approach for imaging a plasmon is energy-filtered transmission electron microscopy in the low-energy-loss region of EELS experiments. By varying the position of the high energetic electron beam one can once again map out the evanescent fields. This was first demonstrated by [107] and [108] in 2007, see Fig. 5.2.

The dispersion relation of a free electron does not cross the light cone at any point [23], so at first it is not obvious how to couple an electron with light. Besides nonlinear 


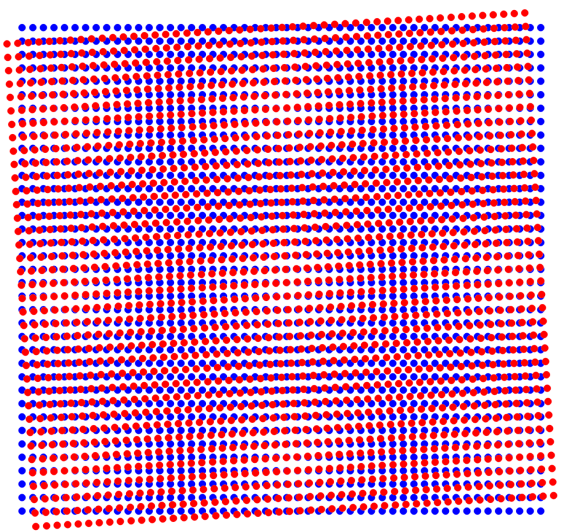

Moiré effect for MNPs and fluorophores:

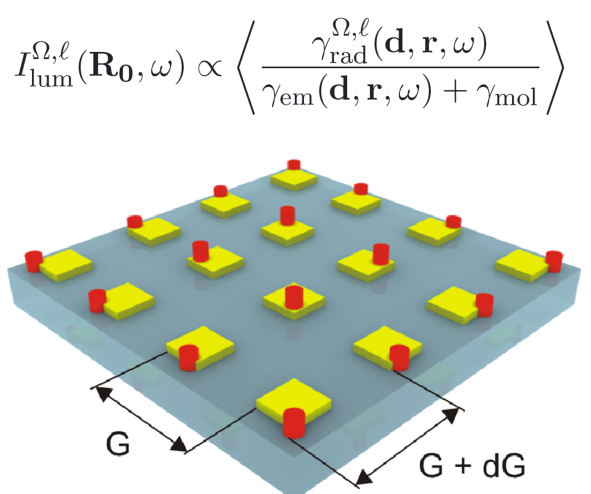

Figure 5.1.: Principal scheme of optical Moiré effect and the discussed MNP and fluorophore arrays lying upon another together with Eq. (10.1). For a detailed discussion see Chap. 10.

(a)

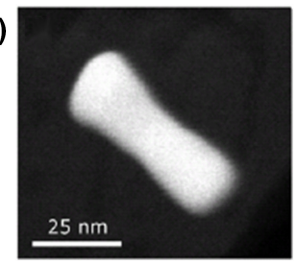

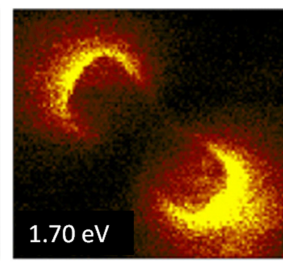

(b)

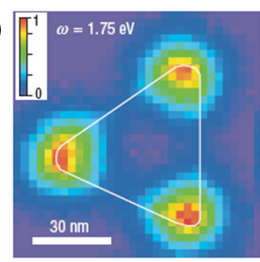

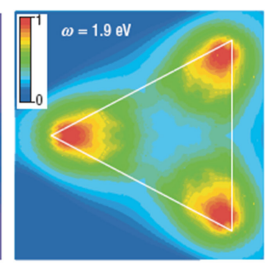

Figure 5.2.: First reported plasmon mapping with EELS experiments. Figures taken from [107] (a) and [108] (b).

processes or electrons with less momentum than their free counterpart (e.g. from photoemission) it is particularly the interaction with light with higher momentum (evanescent fields) that allows the interaction.

The high-energetic electrons move with $70-80 \%$ of the speed of light and thus relativistic effects come into play. During one plasmon oscillation the swift electrons are able to move about $100 \mathrm{~nm}$ - this is sufficient for the plasmon-electron interaction, a process reminiscent of a self energy schematically shown in Fig. 5.3. In a quantum picture, the electron loses energy through the excitation of a surface plasmon. In the classical picture the electron has to perform work against the field of the excited plasmon and thus loses a little bit of its energy. In [109] it is shown that a quantum mechanical description of EELS yields the same results as a semiclassical formalism if all the inelastic signal is collected [23]. This allows us to simply incorporate the 
relativistic motion of the electrons to our BEM approach in terms of the LiènardWiechert potentials. We calculate the induced electric field at the positions of the electron beam and can express the energy-loss probability through the work against this field, see Eq. (11.1).

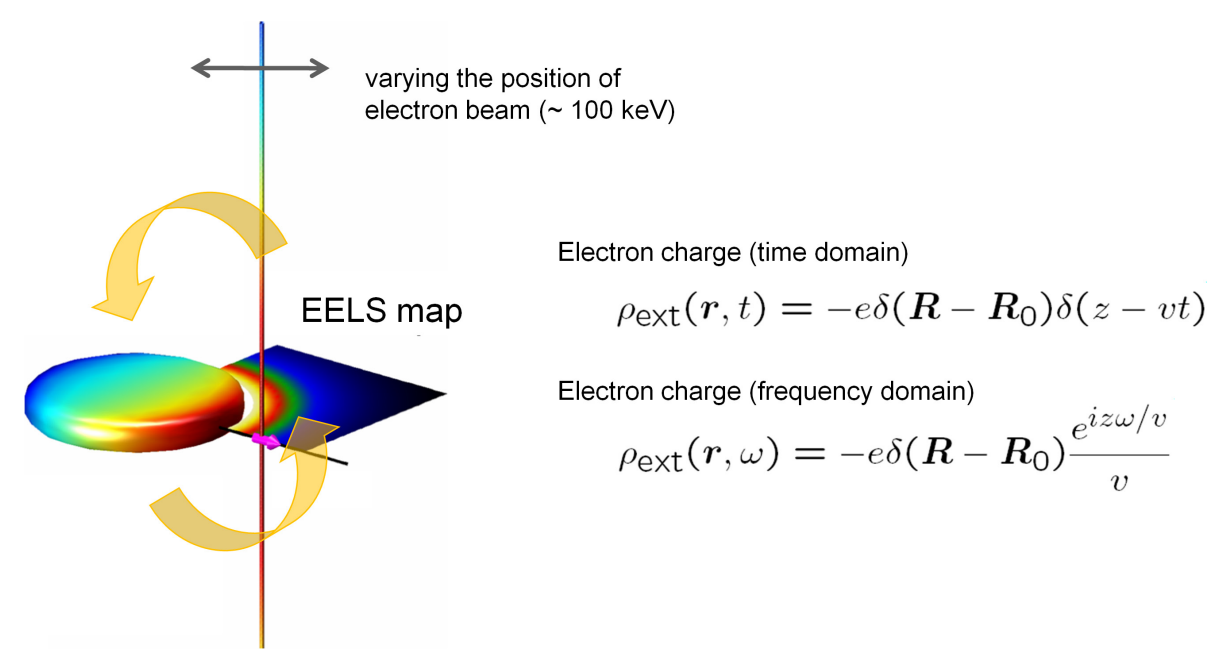

Figure 5.3.: Principle of EELS interaction: The electron beam excites a surface plasmon which in turn acts back on the electrons at a later position. The expression of the electron charge $\varrho_{\text {ext }}$ corresponds to a charged wire.

In the time domain the electron beam interacts with a surface plasmon oscillating in time, whereas in the frequency domain the plasmon oscillation becomes frozen and the electrons interact with a periodically modulated charge distribution as indicated at the expressions for the electron charge in Fig. 5.3. It is important to notice that we get a nonlocal interaction here, the electron at position $r_{e}$ induces the plasmonic field which acts back on the electron at a later time at position $r_{e}^{\prime}$. In this sense the energy loss probability can be expressed through the integral over the imaginary part of the induced Green tensor $[110,111]$ at the beam position $r_{0}=\left(x_{0}, y_{0}\right)$ :

Electron energy loss probability in terms of Green function

$$
\Gamma\left(\boldsymbol{r}_{0}, \omega\right) \propto \int d t d t^{\prime} \Im m\left\{e^{i \omega\left(t^{\prime}-t\right)} G^{\text {ind }}\left[\boldsymbol{r}_{e}(t), \boldsymbol{r}_{e}\left(t^{\prime}\right) ; \omega\right]\right\},
$$


which directly follows from Eq. (11.1). As discussed in Chap. 11 we calculated the energy loss for a rod-shaped particle in comparison to STEM EELS and EFTEM measurements performed by the FeLMI group at the Technical University Graz. In Fig. 5.4 we show the results of experiment and theory, which once again match quite nicely.
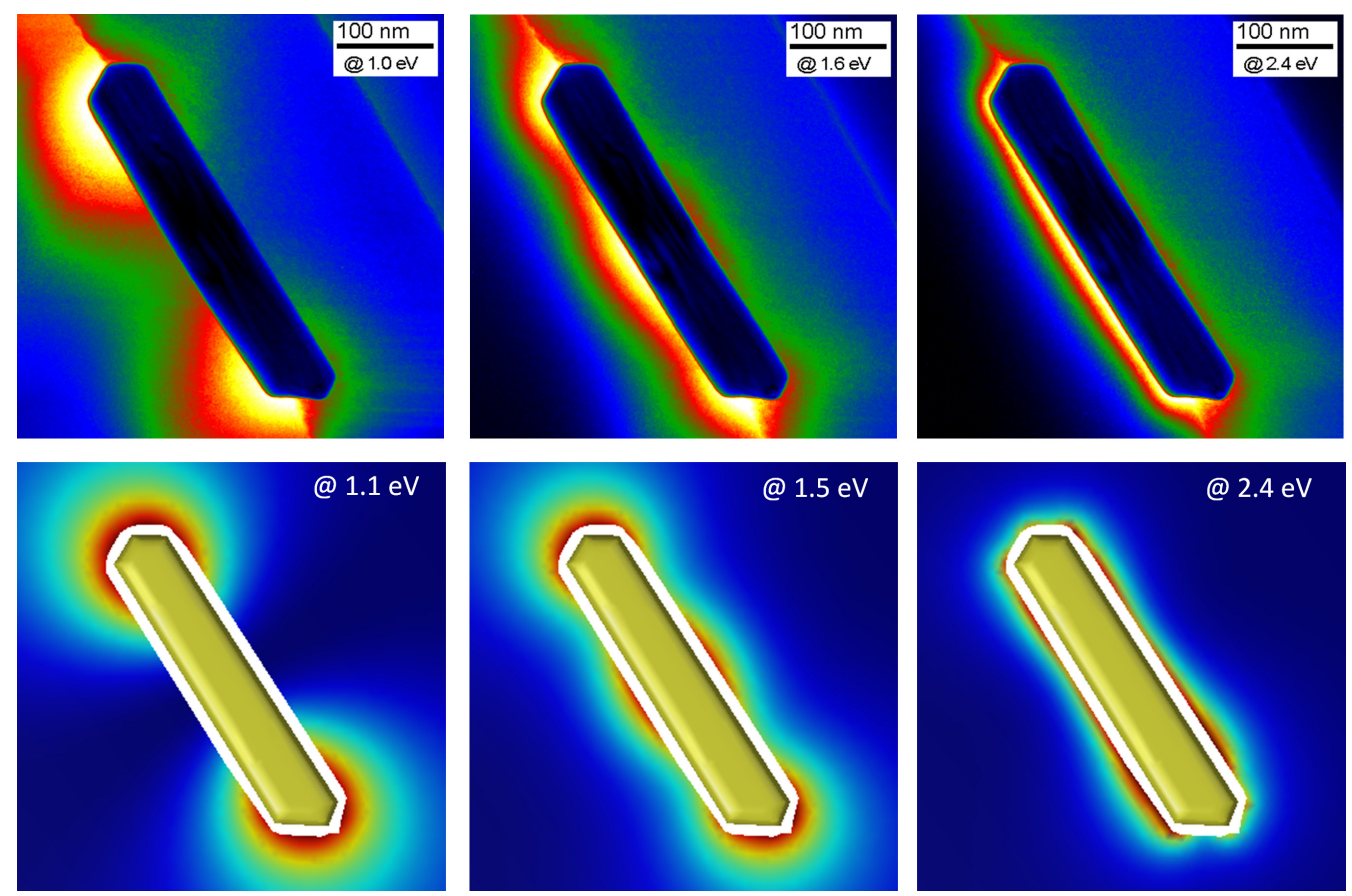

Figure 5.4.: Comparison of measured and simulated EFTEM maps as described in Chap. 11. On the left we see the plasmon dipole mode with an energy of about 1 $\mathrm{eV}$, in the middle the quadrupole mode at around $1.5 \mathrm{eV}$ and the octupole mode at $2.4 \mathrm{eV}$ on the right.

There has been some discussion if the EELS signal directly renders the photonic LDOS $[110,111]$. It turns out, that - although intimately related - there is no direct link between EELS and LDOS maps, and that EELS can even be blind to hot spots in the gap between coupled nanoparticles [110].

For the bonding plasmon mode plotted on the left side of Fig. 5.5 the fields in the gap region are very strong, resulting in a very high LDOS. The EELS signal on the contrary is completely blind to the hot spot. For the antibonding mode on the right hand side the behavior changes to the opposite: Because of the vanishing fields the 


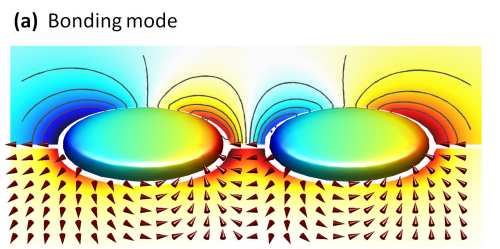

(b) Antibonding mode
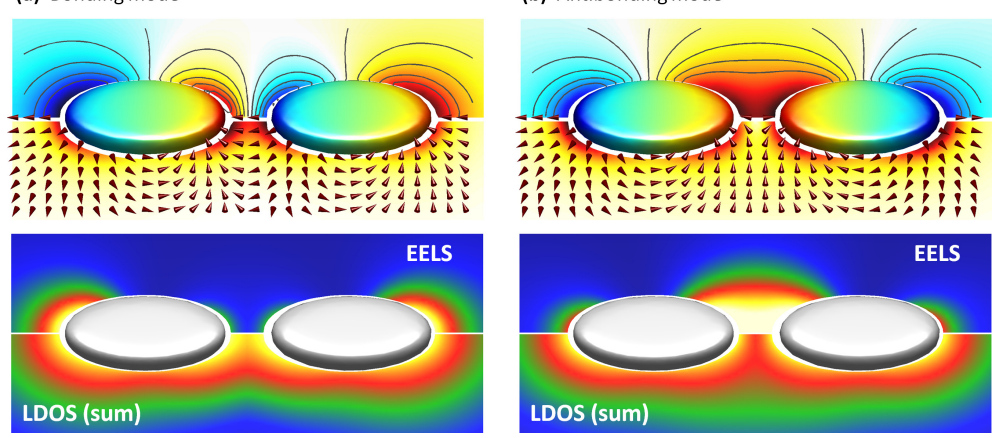

Figure 5.5.: The lower panels show EELS and LDOS maps for the bonding (a) and antibonding modes (b) of coupled nanoparticles. In the upper panels the corresponding scalar potential and electric field is plotted. As discussed in [110], the EELS signal is connected to the scalar potential whereas the photonic LDOS measures the electric field.

LDOS drops in the gap, whereas the EELS rate has a pronounced maximum [110]. 


\section{Additional remark}

Why is the kinetic energy of the electrons in EELS experiments so high? In fact, surface plasmons can also be excited by electron beams with lower energies (e.g. reflection EELS experiments ${ }^{3}$ with energies of 1000-2000 eV). But in transmission EELS the electron has to pass through the material and the smaller the electron wavelength the better the resolution, see Fig. 5.6.

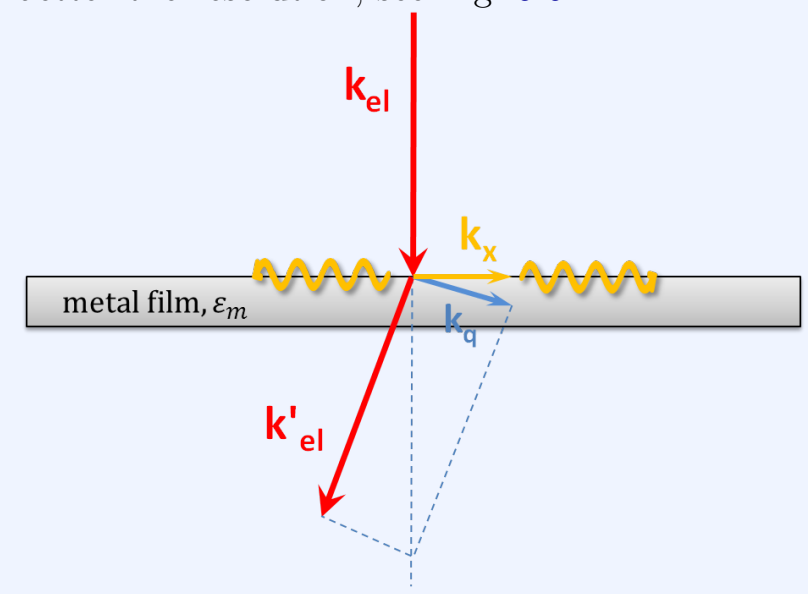

Figure 5.6.: Excitation of surface plasmons through the transmission of swift electrons in thin films $[21,22]$. The incoming wave vector $\boldsymbol{k}_{\mathrm{el}}$ transfers the component $\boldsymbol{k}_{q}$ to the thin film. The energy of the surface plasmon is determined by the projection $\boldsymbol{k}_{x}$ to the surface and the dispersion relation 2.6.

Note that for particle plasmons there is no momentum conservation, see Chap. 2.

\footnotetext{
${ }^{3}$ In 1956 Powell [30] investigated inconsistencies in the EELS spectra of transmission and reflection EELS experiments which lead to the experimental verification of surface plasmons.
} 



\section{Influence of surface roughness}

It is nearly impossible to fabricate perfectly smooth nanoparticles, thus it is quite important to discuss the influence of surface roughness on the optical properties of MNPs. Especially if e-beam lithography (see App. B.2) is used to produce the particles, the resulting metal structures are polycrystalline and the surfaces are quite rough. Contrary to our first guess we found out that a moderate amount of surface roughness has no significant impact on the far-field optical properties of MNP. In Chap. 12 we show that this behavior can be interpreted as some kind of plasmonic averaging over the random height fluctuations of the rough metal surface (motional narrowing).

\subsection{Generation of a rough particle in the simulation}

To allow systematic investigations of surface roughness on metallic nanoparticles, we need to control and quantify the "amount" of roughness on a surface. One possibility to do this is to add (controllable) stochastic height variations to the smooth surface of an ideal nanoparticle. Let us exemplify the idea for the $2 \mathrm{D}$ case: We assume that the height of each vertex of a triangulated plate is given by a function $h(x, y)$. The mean value should vanish $\langle h(x, y)\rangle=0$ and the value of $h$ at $\left(x^{\prime}, y^{\prime}\right)$ should be correlated to the value at position $(x, y)$ through a distance dependent function: $\left\langle h(x, y) h\left(x^{\prime}, y^{\prime}\right)\right\rangle=f\left(x-x^{\prime}, y-y^{\prime}\right)$. This requirements are all fulfilled if we introduce a Gaussian autocorrelation function and attach arbitrary phase factors to all Fourier coefficients. In this way we are able to average the z-coordinate of each vertex corresponding to this random potential and we have two parameters to control the roughness, the standard deviation of the Gaussian function $\varsigma$ (determines 
the amount of roughness) and a height scaling parameter $\Delta h$ (assigns the deepness of the asperity).

Wrapping the created roughness around the surface of a nanorod, we get the structure shown in Fig. 6.1.
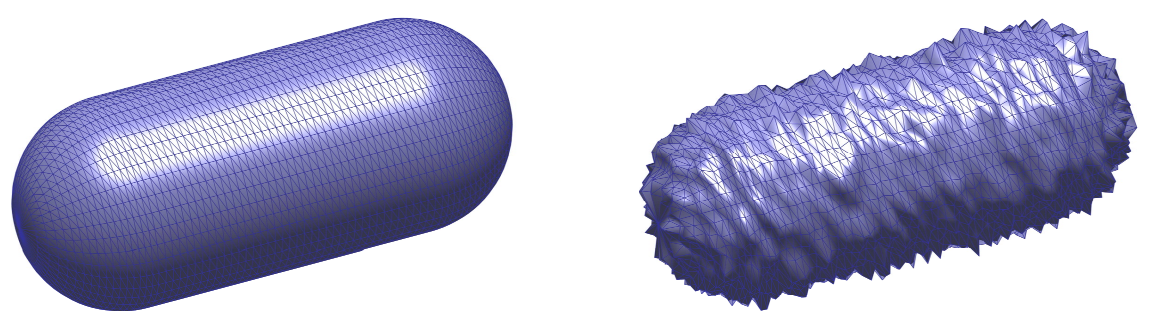

Figure 6.1.: Triangulated nanorod with smooth surface and stochastic height variations generated with a random Gaussian potential. Problems in the simulation may arise if sharp features are present.

Unfortunately very sharp features in the triangulated mesh may lead to inaccuracies in the simulation and may cause diverging surface charge densities. Luckily we get an even better result without sharp peaks, if we do not directly interpolate the height of each vertex with the Gaussian autocorrelation, but rather generate a box around the particle and interpolate the stochastic vertex height with respect to this box, see Fig. 6.2.
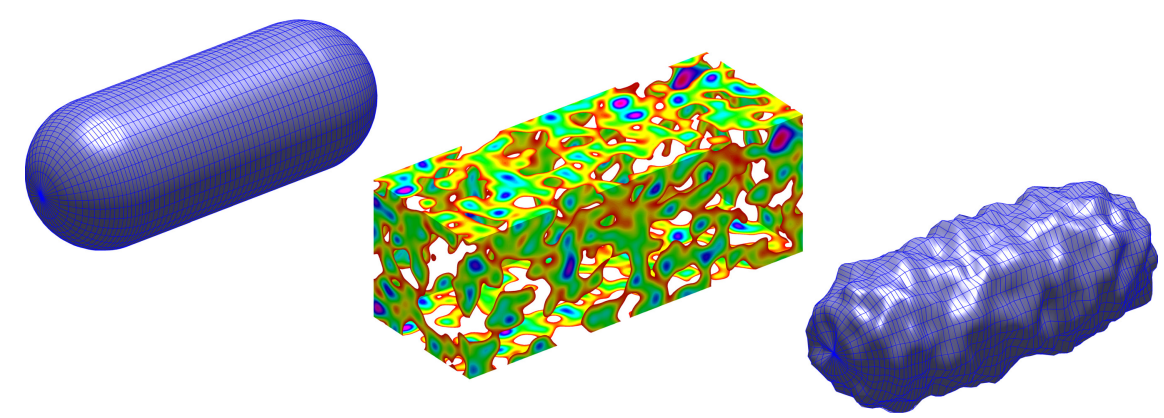

Figure 6.2.: Generating a box around the smooth particle and interpolating stochastic vertex heights with respect to this box gives a much better result without sharp features as in Fig. 6.1. The random height variations are again controllable by parameters of the Gaussian autocorrelation, see Eq. (6.1).

Again we use a normal probability density function multiplied with $N$ random phases 
$e^{i \phi_{\text {rnd }}}$ (for each spatial direction)

\section{Generation of surface roughness}

$$
h(x, y, z)=\frac{1}{N} \Re e\left\{\mathcal{F}^{-1}\left[e^{-\frac{1}{2} \sigma^{2}\left(k_{x}^{2}+k_{y}^{2}+k_{z}^{2}\right)} e^{i \phi_{\mathrm{rnd}}}\right]-\frac{1}{2}\right\}
$$

where $\mathcal{F}^{-1}$ denotes the inverse Fourier transform, $N$ is a normalization factor and $\varsigma^{2}$ is the variance of the height fluctuations. For the arbitrary phase factors $\phi_{\text {rnd }}$ the MATLAB $^{\oplus}$ built-in function rand(n) to create uniformly distributed pseudorandom numbers has been used.

We next interpolate the scaled stochastic height variations $\Delta h \cdot h(x, y, z)$ to the nanoparticle surface and displace the vertices of the nanoparticle along the surface normal directions. The influence of $\Delta h$ and $\varsigma$ is shown in Fig. 6.3.

Constant height scaling $\Delta h$, varying variance $\varsigma$
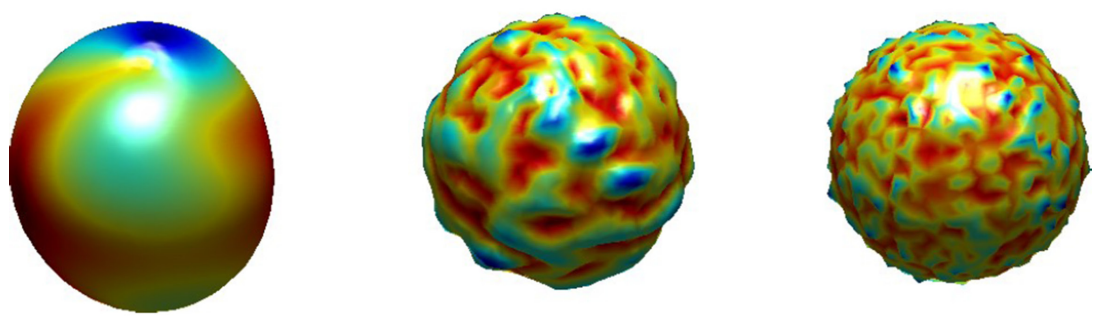

Constant variance $\varsigma$, varying height scaling $\Delta h$
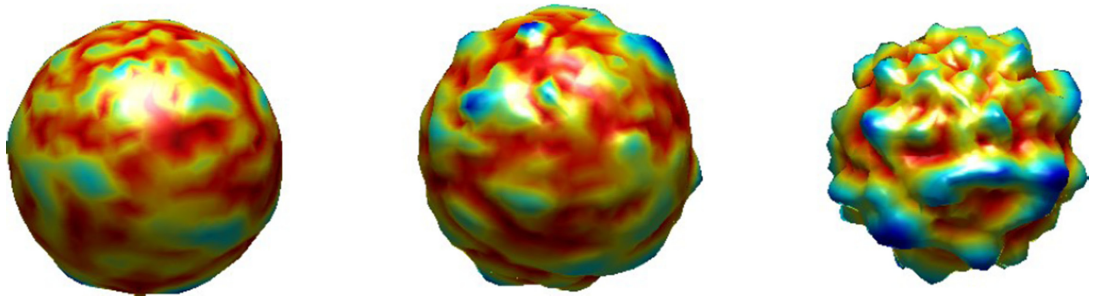

Figure 6.3.: Surface roughness generated with Eq. (6.1) for changing parameters. 
Another possibility for the simulation of "realistic" particles is based on the extraction of their contour out of scanning electron microscope (SEM) images, see Fig. 12.1. The triangulated particle is generated by assigning a certain height profile (with a rounded transition to the top and bottom area, see [84]) to the extracted contourpolyline and wrapping a mesh around the structure. A SEM image together with the extracted contour and the final BEM particle is shown in Fig. 6.4.
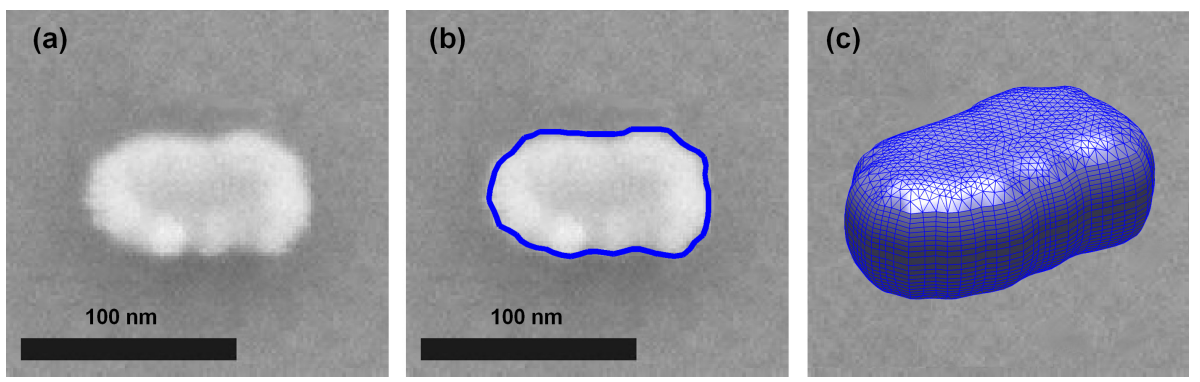

Figure 6.4.: (a) SEM image of a rod-shaped nanoparticle fabricated by e-beam lithography (see Appendix B.2). (b) Contour extraction and (c) creation of the corresponding triangulated particle for a BEM simulation. 


\subsection{Theoretical analysis of surface roughness}

Let us for one moment take only the mean value of the height difference of bumps or hollows at a rough surface as an indicator for the degree of roughness. For a medium sized nanoparticle this mean value is of the order of nanometers. One possibility to describe the optical properties of structures with such small features ${ }^{1}$ is presented in [112], for example. By writing the dielectric constant $\varepsilon(\boldsymbol{k}, \omega)$ as a function of both the wave vector $\boldsymbol{k}$ and the angular frequency $\omega$, the authors incorporate the effect of small features via the spatially nonlocal response of materials to Maxwell's equations ${ }^{2}$

On the other hand we can also understand the influence of rough surfaces if we once again consider the eigenmode expansion discussed in Sec. 3.3.2. We can model the rough surface as a distortion of the surface $\partial \Omega$ from its ideal shape, see Chap. 12. The surface derivative of the Green function $F$ then changes to $F+\delta F$.

We want to determine, how the peak position of the plasmons is affected in case of surface roughness and since this peak position is assigned by the plasmon energy, we need to investigate the modification of the Eigenvalue $\lambda_{k}$. For sufficiently small $\delta F$ we can employ a perturbation analysis, where we treat $F$ as the unperturbed part and $\delta F$ as the "perturbation". This calculation is carried out in Sec. 12.4, and the result is shown in Fig. 12.3.

Our findings show a surprisingly small effect for the influence of surface roughness on the position and width of the plasmon peaks of metallic nanoparticles. We identified the reason for this as motional narrowing ${ }^{3}$, where the plasmon averages over the random height fluctuations $h$.

\footnotetext{
${ }^{1}$ So-called finite size effects have been phenomenologically accounted for by increasing the damping rate of the conduction electrons contribution to the permittivity, see [44].

${ }^{2}$ Their implementation is based on the self-consistently solved hydrodynamic Drude model

${ }^{3}$ This behavior is known from electron-hole pairs in semiconductor quantum wells [113], where the propagating excitons "average" over the random potential of local monolayer fluctuations, which results in a narrowing of the exciton lineshape.
} 



\section{Nonlinear optical effects of plasmonic nanoparticles}

The light-matter interaction between MNPs and an electromagnetic wave happens on a very fast time scale and, as we have discussed in Sec. 2.4, already after a few femtoseconds the plasmonic excitations start to vanish again. The temporal evolution and this ultrafast relaxation of surface plasmon polaritons is of central importance for many kinds of plasmonic applications.

It is amazing that we can explore such rapid dynamic processes with experiments nowadays. One way to enter the ultrafast world of plasmon dephasing is given by nonlinear autocorrelation measurements, which allow to determine sub-10 fs decay times. Usually a bandwidth-limited laser pulse working in the few-cycle regime is used to excite nonlinear effects which serve as a non-invasive monitor for the plasmon dynamics [46].

The simplest nonlinear effect is second-harmonic generation (SHG), where a nonlinear material absorbs two photons of frequency $\omega$ and emits a new photon of frequency $2 \omega$. But because of the inversion symmetry in the atomic arrangement of metals, SHG is forbidden in the bulk $[46,114,115]$. However the symmetry is broken at the surface and so a second order dipole response can exist in the surface region. There can be electric dipolar surface contributions due to the broken symmetry or also higher order bulk signals can contribute the second-order nonlinearity [46], but the latter are known to be much weaker and can therefore be neglected.

Breaking the symmetry of centrosymmetric media also allows a second-order signal for a perfectly symmetric nanosphere for example, it will just strongly depend on defects, facets and other small deviations from the spherical shape [116]. This defect dependence will give a huge varying signal for different particles of the same shape. Another example of SHG measurements for systems with a broken symmetry has 
been reported by [117], where they investigated arrays of noncentrosymmetric Tshaped gold nanodimers.

On the other hand third-harmonic generation (THG), the next higher nonlinear process where a mixing of four fields $\left(\boldsymbol{E} \propto \boldsymbol{\chi}^{(3)} \boldsymbol{E}^{3}\right)$ occurs, is allowed in all media and not restricted to symmetry considerations, see Fig. 7.1. Supported by the above mentioned reasons the $\chi^{(3)}$ nonlinearity for gold nanoparticles is also much stronger than the corresponding SHG signal [78], which makes THG a perfect candidate to investigate the femtosecond dynamics of particle plasmons.

(a)

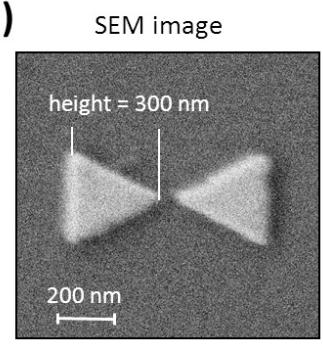

(b)

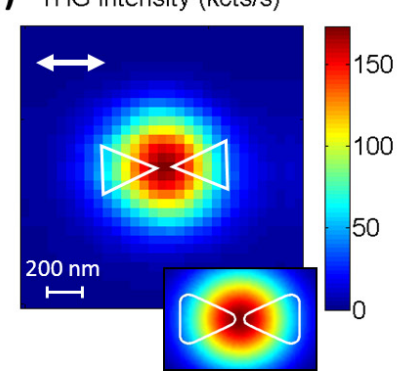

(c) THG intensity (kcts/s)

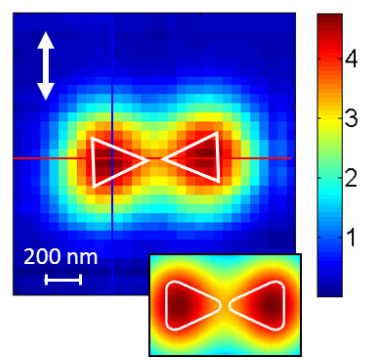

Figure 7.1.: (a) SEM image of a gold bowtie nanoantenna. (b) and(c) measured THG intensity for linear polarization along long and short axis respectively. The lower insets show the corresponding simulation results, further details see Chap. 13. (Measurements by Tobias Hanke, University of Konstanz.)

The first observation of third-harmonic signals from individual gold colloids down to $40 \mathrm{~nm}$ diameter can be found in [116] and a phenomenological macroscopic theory of optical second- and third-harmonic generation from cubic centrosymmetric crystals has been discussed by [118]. 


\subsection{Autocorrelation}

To measure the autocorrelation the sample is excited by two sequenced laser pulses with a varying time delay $t^{\prime}$ between the pulses. The collected intensity is given by the interfering electric fields to the power of four (second order autocorrelation $G_{2}$ ) or to the power of six (third order autocorrelation $G_{3}$ ) and averaged over the detector response time $T[46]$ :

\section{Second and third order autocorrelation function}

$$
\begin{aligned}
\text { SHG: } & G_{2}\left(t^{\prime}\right)=\frac{1}{T} \int_{-T}^{+T}\left[E(t)+E\left(t-t^{\prime}\right)\right]^{4} d t, \\
\text { THG: } & G_{3}\left(t^{\prime}\right)=\frac{1}{T} \int_{-T}^{+T}\left[E(t)+E\left(t-t^{\prime}\right)\right]^{6} d t .
\end{aligned}
$$

This pulse interference can also be described by a simple harmonic oscillator model (see [46]). By inserting harmonic fields in the above expressions for $G_{2}$ or $G_{3}$ the characteristic peak ratio of 1:8 for SHG or 1:32 for THG follows directly from the in and out of phase expression of the electric fields. In Fig. 7.2 the measured and simulated result of $G_{3}$ for three different nanoantennas is shown.

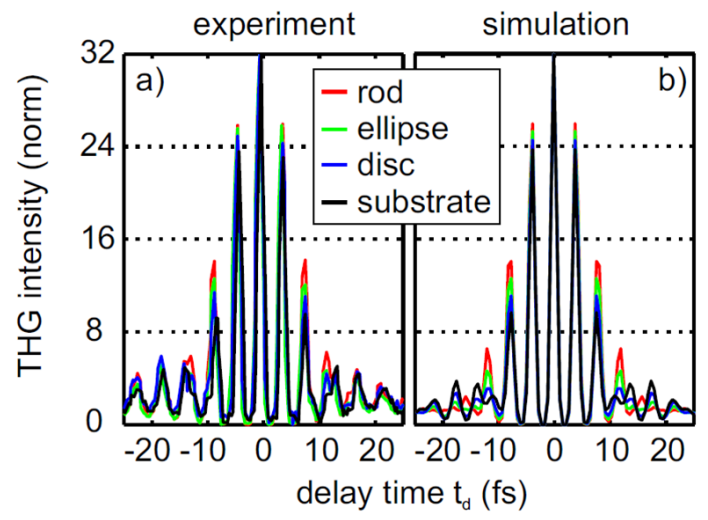

Figure 7.2: Third order autocorrelation for three different nanoantenna geometries, see Tab. 13.1 for spatial dimensions and further details. (a) Experimental and (b) simulation result. (Figure by Tobias Hanke, University of Konstanz.) 


\subsection{Third harmonic imaging}

In Chap. 13 we discuss the third harmonic emission of five different antenna structures. We show, that the THG intensity differs significantly for the different structures and that the knowledge of the plasmon damping time (obtained from autocorrelation measurements) suffices, to explain the differences. The principal scheme for this experiment is depicted in Fig. 7.3. An array of nanoantennas (varying in size with different gap distances) gets illuminated by a laser pulse and the nonlinear emission intensity is collected.

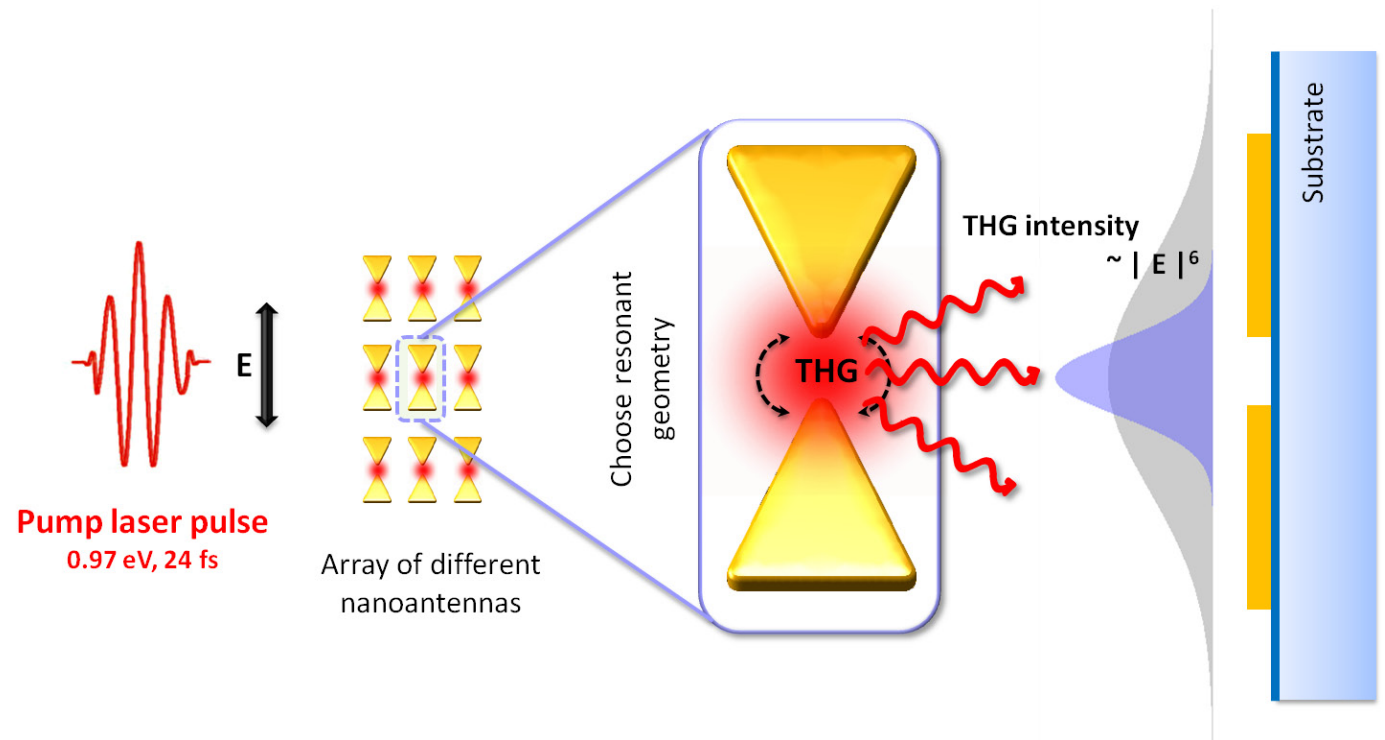

Figure 7.3.: Principle of third harmonic imaging as described in Chap. 13. The few-fs pulse of a pump laser excites a nanoantenna and the nonlinear emission gets collected. By raster scanning the laser pulse over a sample of varying antenna geometries the most resonant structure for this excitation can be picked - see Tab. 13.1.

Anyhow, also the dielectric environment of the metallic particles can contribute to the nonlinear emission, but the susceptibility of air or fused silica is at least several orders of magnitude weaker than the one for gold: 


$$
\begin{aligned}
& \chi^{(3)} \approx 10^{-11} \text { esu for gold [119], } \\
& \chi^{(3)} \approx 10^{-14} \text { esu for fused silica [120], } \\
& \chi^{(3)} \approx 10^{-18} \text { esu for air [121]. }
\end{aligned}
$$

Nonlinear emission spectroscopy emerges as a new powerful tool for the spatiotemporal characterization of nanoantennas. As described in Chap. 13, we demonstrate that not the shape but the volume of the nanoantennas plays a crucial role for the determination of the nonlinear emission intensity. Due to radiative damping the structure with the lowest active volume generates by far the strongest THG emission.

In [116] it has been stated that THG signals also serve as label for bio-sensors and may be used for the tracking of single molecules. Placing an emitter in the gap region of the nanoantennas (see Fig. 7.4 may also be one of the next steps for our analysis (also see [57]).

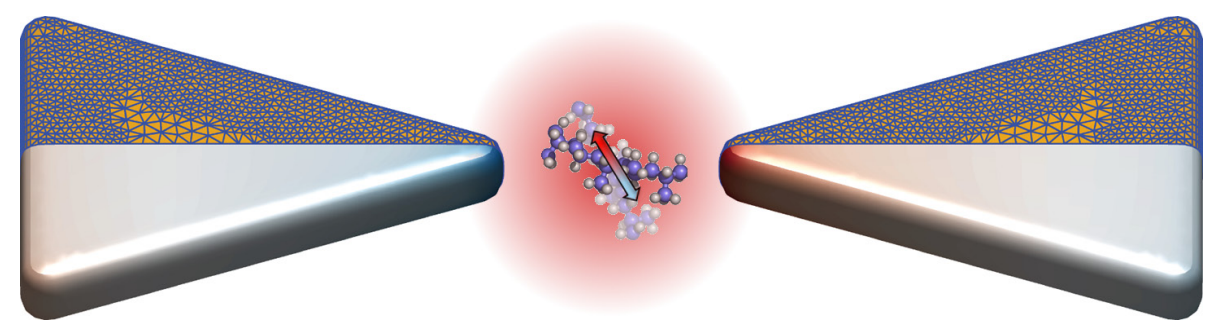

Figure 7.4.: Schematic representation of a bowtie nanoantenna used as one of five different antenna geometries in Chap. 13. One half of the antenna shows the surface charge distribution at the resonance energy (the bonding mode is clearly visible), whereas the other half depicts the surface discretization needed for the simulation with the BEM. 

Part II.

\section{Paper reprints}



In the next chapters reprints of the following papers are included:

- Jan Becker, Andreas Trügler, Arpad Jakab, Ulrich Hohenester, and Carsten Sönnichsen

The Optimal Aspect Ratio of Gold Nanorods for Plasmonic Bio-sensing Plasmonics 5 (2010) 161

- Arpad Jakab, Yuriy Khalavka, Jan Becker, Andreas Trügler, Christina Rosman, Ulrich Hohenester, and Carsten Sönnichsen

Highly sensitive plasmonic silver nanorods submitted (2011)

- Daniel Koller, Ulrich Hohenester, Andreas Hohenau, Harald DitlBacher, Frank Reil, Nicole Galler, Franz Aussenegg, Alfred Leitner, Andreas Trügler, and Joachim Krenn Superresolution Moiré Mapping of Particle Plasmon Modes Physical Review Letters 104 (2010) 143901

- Bernhard Schaffer, Ulrich Hohenester, Andreas Trügler, and FERdinAND Hofer

High-Resolution Surface Plasmon Imaging of Gold Nanoparticles by Energy Filtered Transmission Electron Microscopy

Physical Review B 79 (2009) 041401(R)

- Andreas Trügler, Jean-Claude Tinguely, Joachim Krenn, Andreas Hohenau, and Ulrich Hohenester

Influence of surface roughness on the optical properties of plasmonic nanoparticles

Physical Review B 83 (2011) 081412(R) 
- Tobias Hanke, Julijan Cesar, Vanessa Knittel, Andreas Trügler, Ulrich Hohenester, Alfred Leitenstorfer, and Rudolf Bratschitsch Tailoring spatiotemporal light confinement in single plasmonic nanoantennas submitted (2011)

Other parts of this work that are not included here are published in:

- Ulrich Hohenester and Andreas Trügler MNPBEM - A Matlab toolbox for the simulation of plasmonic nanoparticles submitted to Computer Physics Communications (2011)

- Jürgen Waxenegger, Andreas Trügler, and Ulrich Hohenester Mapping exciton wavefunctions in semiconducting carbon nanotubes with plasmonic nanoparticles to appear in Physical Review B (2011)

- Andreas Trügler and Ulrich Hohenester Strong coupling between a metallic nanoparticle and a single molecule Physical Review B 77 (2008) 115403

- Ulrich Hohenester and Andreas Trügler Interaction of single molecules with metallic nanoparticles IEEE Journal of Selected Topics in Quantum Electronics 14 (2008) 1430 


\title{
8. PAPER: The Optimal Aspect Ratio of Gold Nanorods for Plasmonic Bio-sensing
}

\author{
Jan Becker ${ }^{1}$, Andreas Trügler ${ }^{2}$, Arpad Jakab ${ }^{1}$, Ulrich Hohenester ${ }^{2}$, \\ and Carsten Sönnichsen ${ }^{1}$ \\ ${ }^{1}$ Institute of Physical Chemistry, University of Mainz, Jakob-Welder-Weg 11, 55128 Mainz, Germany \\ ${ }^{2}$ Institut für Physik, Karl-Franzens-Universität Graz,Universitätsplatz 5, 8010 Graz, Austria
}

Published in: Plasmonics 5, 161 (2010)

\begin{abstract}
The plasmon resonance of metal nanoparticles shifts upon refractive index changes of the surrounding medium through the binding of analytes. The use of this principle allows one to build ultra-small plasmon sensors that can detect analytes (e.g., biomolecules) in volumes down to attoliters. We use simulations based on the boundary element method to determine the sensitivity of gold nanorods of various aspect ratios for plasmonic sensors and find values between 3 and 4 to be optimal. Experiments on single particles confirm these theoretical results. We are able to explain the optimum by showing a corresponding maximum for the quality factor of the plasmon resonance.
\end{abstract}




\subsection{Introduction}

Gold nanoparticles have a long history as optical or electron microscopy labels. More recently, their plasmon resonance has been employed for more elaborate optical nanoscopicsensing schemes. The plasmon resonance of coupled particles depends on interparticle distance [122-125], and the strong plasmonic light scattering efficiency allows for the visualization of single nanorods, for example for orientation sensing [126-129]. Furthermore, the resonance position is influenced by the particle charge $[130,131]$ and the refractive index of the particle's immediate environment [51]. The plasmonic sensitivity to the immediate dielectric environment of the particles allows one to monitor the dielectric constant of liquids and binding events of molecules to the gold particle surface. Here, the nanoparticle sensor acts in a way similar to sensors exploiting the surface plasmon resonance on gold films, which is a standard method in many laboratories. However, whereas the detection scheme for surface plasmon resonance is usually a shift in the plasmon excitation angle [132], plasmonic nanoparticles show a shift in the plasmon resonance frequency [133]. The main advantage of using nanoscopic particles as sensors instead of metal films is their extremely small size which allows one, in principle, to measure analytes in volumes as small as attoliters [134]. The key factor for taking advantage of the small detection volume of plasmonic particles is the single particle plasmon-scattering spectroscopy $[133,135]$. Single particle measurements probe the local environment around one specific particle, which - in principle - enables massive parallelization of nanoparticle plasmon sensors either for analyzing different analytes or obtaining statistics. Recently, we demonstrated a scheme that can conduct parallel sensing on randomly arranged nanoparticles using a liquid crystal device as an electronically addressable entrance shutter for an imaging spectrometer [136].

For such dielectric plasmon nanoparticle sensors, one hopes to have a large spectral shift for a given amount of analyte or refractive index change of the environment. Initially, spherical gold particles were used $[134,137]$ but were soon replaced by gold nanorods [138] due to their higher sensitivity on refractive index changes [139]. A lot of effort has been applied towards identifying the ideal plasmon sensor with a large spectral shift for a given change in refractive index, for example, using rodshaped gold nanorattles [140], metamaterials [141], silver-coated gold nanorods [142] 
and others. However, rod-shaped nanoparticles remain popular for plasmonic applications. Some reasons for this are the ability to fabricate gold nanorods in high quality using seeded crystallization from solution, the adjustability of the plasmon resonance by varying the aspect ratio, the strong scattering efficiency, and the low plasmon damping in nanorods.

Here, by means of simulations and experiments, we investigate which aspect ratio (AR) of gold nanorods is ideal for plasmonic sensing by employing various measures for 'ideal' behavior. There are several different quantities that describe the performance of a plasmonic structure for sensing applications on a single particle level and all of them have their merits for certain applications. We will discuss the most important of them in the following paragraphs - the plasmonic sensitivity to refractive index change as well as various 'figures of merit' - and present their dependency on nanorods' geometry from calculations for spherically capped gold rods with the Boundary Element Method (BEM)) [82,83]. We confirm the identified trends by experimental results obtained using single particle dark field scattering spectroscopy.

\subsection{Plasmon sensor quality}

Changing the refractive index $n$ of the embedding medium by a given amount, $d n$ shifts the plasmon resonance position in wavelength or energy units $\left(\lambda_{\text {res }}, E_{\text {res }}\right.$, respectively, see Fig. 8.1 a). The corresponding proportionality constant or sensitivity $S_{\lambda}$ (often simply denoted $\Delta \lambda / \mathrm{RIU}$ ) can be expressed in wavelength $\left(S_{\lambda}\right)$ or energy $\left(S_{E}\right)$ units [143]: $S_{\lambda}=d \lambda_{\mathrm{res}} / d n$ and $S_{E}=d E_{\mathrm{res}} / d n=S_{\lambda} \frac{d E}{d \lambda}=-S_{\lambda} / \lambda^{2} \times 1,240 \mathrm{~nm} / \mathrm{eV}$.

The relatively broad plasmon linewidth $\Gamma$ (full width at half maximum) complicates the analysis further, because the plasmon linewidth of nanostructures with different geometries can vary more than tenfold. Since it is easier to detect a given resonance shift for narrow lines, the resonance shift relative to the linewidth is a more meaningful measure of the sensoric quality. This dimensionless quantity is often referred to as the 'figure of merit' $(\mathrm{FOM})=S / \Gamma[144]$.

The FOM is easily determined experimentally and allows for the comparison of the plasmonic properties of many different structures with a single sharp plasmonic resonance. For more complex plasmonic responses (such as in metamaterial structures 
based on an analog of electromagnetically induced transparency (EIT) [141]), where the plasmon resonance does not follow a simple Lorentz peak shape, the concept needs to be refined. In practice, one would normally detect a spectral shift of a resonance as a relative intensity change $d I / I$ at a fixed wavelength $\lambda_{0}$ induced by a small index change $d n$. We can therefore define an alternative dimensionless figure of merit:

$$
\mathrm{FOM}^{*}=\left(\frac{(d I / d n)}{I}\right)_{\text {max }}=\left(\frac{S_{\lambda}(d I / d \lambda)}{I}\right)_{\text {max }} .
$$

The wavelength $\lambda_{0}$ is chosen such that $\mathrm{FOM}^{*}$ has a maximum value - for gold nanorods in the shoulder of the resonance of the long-axis plasmon near the place where the slope $d I / d \lambda$ is highest.

Bio-sensing applications are even more complex. In this case, one seeks to detect the binding of small (organic) molecules to the nanoparticle surface instead of exchanging the entire embedding medium. The spectral shift now depends on the relative size of the molecules to the volume the plasmon field penetrates into the medium. Furthermore, the sensitivity is reduced with increasing distance to the particles surface. A 'figure of merit' trying to capture the different sensing volumes of various nanostructures can be defined as the $\mathrm{FOM}_{\text {layer }}^{*}$ for a homogeneous coating of molecules with a specific refractive index (for example, $n=1.5$, typical for organic molecules) in a layer of thickness $l$ around the particle normalized to this layer thickness. The formal definition of this 'figure of merit for thin layers' $\mathrm{FOM}_{\text {layer }}^{*}$ is therefore:

$$
\mathrm{FOM}_{\text {layer }}^{*}=\left(\frac{(d I / I)}{d l}\right)_{\text {max }}
$$

To compare the general sensing quality of different nanostructures, the limit of FOM $_{\text {layer }}^{*}$ for $l \rightarrow 0$ gives a defined value. Table 8.1 summarizes the different quantities used to determine the quality of plasmon sensors and their definitions. 


\subsection{Results}

We simulate the light scattering cross-sections of gold nanorods by solving Maxwell's equations using the BEM and tabulated optical constants for gold [41]. Regarding shape, we use rods with spherical end-caps varying the particle length while keeping the diameter constant at $20 \mathrm{~nm}$. Even though the exact end-cap geometry influences the resonance position [145], many researchers have successfully used spherical endcaps for their simulations [146]. We vary the aspect ratio by changing only the particle length because gold nanoparticle synthesis usually results in particles of comparable width. From the BEM calculations, we find a linear relationship between aspect ratio $\mathrm{AR}$ and plasmon resonance wavelength: $\lambda_{\text {res }} / \mathrm{nm}=505+114(\mathrm{AR}-1)$. The calculations of the layer effect for $\mathrm{FOM}_{\text {layer }}^{*}$ were performed within the quasi-static approximation (QSA) for spheroids [147] since we did not implement coated particles in our BEM simulations so far. However, comparisons of QSA with full solutions for the Maxwell equations have shown good qualitative agreement [145].

The plasmon sensitivity in wavelength units $S_{\lambda}$ (calculated with BEM) shows the expected steady increase for an increasing aspect ratio [139] (not shown here), whereas the sensitivity in energy units $S_{E}$ shows a maximum at an aspect ratio of $\mathrm{AR}_{\max }=3$ (Fig. 8.1). Both the 'classical' FOM and the more general FOM* show the same trend with two maxima (Fig. 8.2 a). The classical FOM has a maximum for rods with an aspect ratio of $\mathrm{AR}=4.3$ and a second local maximum at $\mathrm{AR}=3.2$. The maxima of the generalized $\mathrm{FOM}^{*}$ is slightly shifted to rod aspect ratios of $\mathrm{AR}=4.2 / 3.1$ (cf. Table 8.1). The values of the 'figure of merit for small layers' $\mathrm{FOM}_{\text {layer }}^{*}$ (obtained by QSA calculations) are shown in Fig. $8.2 \mathrm{~b}$ for increasing layer thickness $l$ and show maxima at the aspect ratios of $\mathrm{AR}=3.0$ and $\mathrm{AR}=4.3$. The first maximum at $\mathrm{AR}=3.0$ is higher for thin layers and in the limit of layer thickness $l \rightarrow 0$. Hence, rods with aspect ratios in the range of 3 to 4 are the best candidates to investigate changes in the refractive index of the embedding medium.

To verify the theoretical conclusions given above, we compared the theoretical results with experimental values measured on single particles. The particle spectra were obtained for nanorods immobilized on a glass substrate and exposed to liquids with various refractive indices in a dark field microscope coupled with an imaging spectrometer $[135,136]$. The resulting values are shown in Fig. 8.3 and show the 

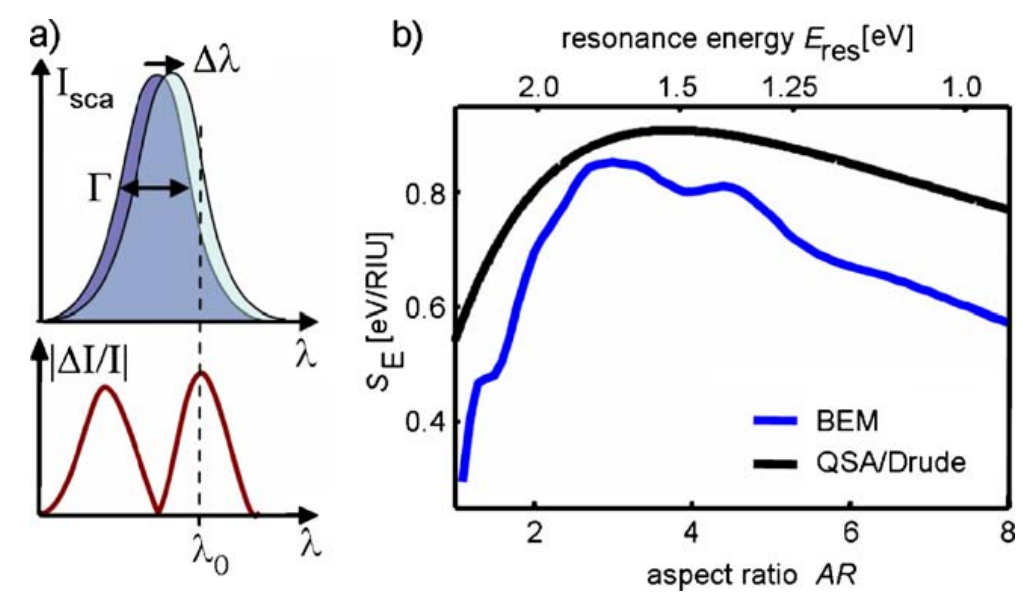

Figure 8.1.: (a) The plasmon resonance shifts $(\Delta \lambda)$ upon changing the refractive index of the surrounding medium by $d n$. To detect such changes, the sensitivity $S_{\lambda}=\Delta \lambda / \Delta n$ needs to be large. Other important parameters that characterize plasmonic sensors are the plasmon linewidth $\Gamma$ and the wavelength $\lambda_{0}$ where the relative intensity change $|\Delta I / I|$ is largest (see text). (b) The plasmonic sensitivity in energy units $S_{E}$ shows a maximum for gold nanorods with aspect ratio AR $=3.0$. The blue line shows data calculated with the BEM, assuming gold rods with a diameter of $20 \mathrm{~nm}$ and spherical end-caps embedded in water $(n=1.33)$. A similar trend is found for a simple calculation using the QSA and a Drude dielectric function for gold (black line).

same trend as predicted by the BEM simulations. The values are generally lower than the calculated values due to the influence of the supporting glass substrate and are potentially also influenced by a thin organic coating of the particles remaining from the synthesis. The variance within the experimental results is not a measurement error but originates from small derivations from the ideal particle geometry and environment. For example, defects in the nanoparticles' crystal structure would increase damping, therefore broadening the plasmon resonance and reducing FOM and $\mathrm{FOM}^{*}$.

\subsection{Discussion}

The existence of a maximum of the plasmon sensitivity in energy units can be derived from a simple calculation of the polarizability of a spheroid in quasistatic approxi- 
a)

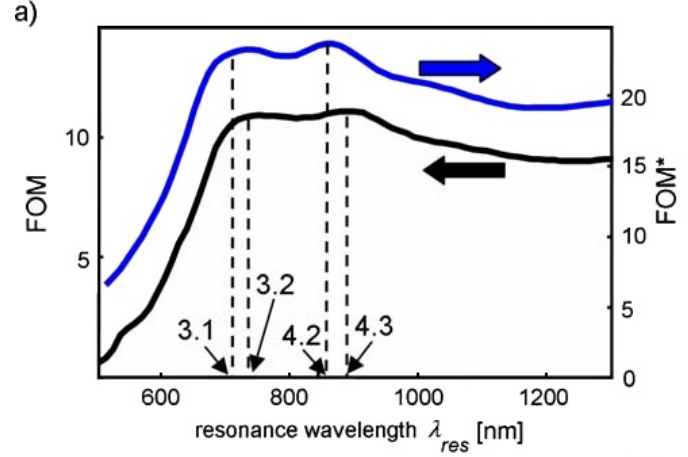

b)

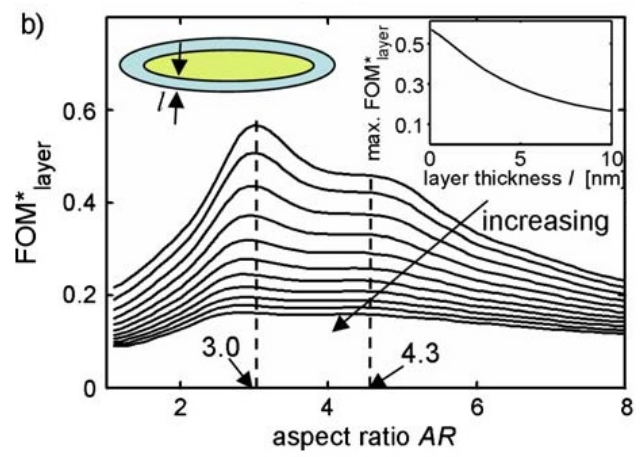

Figure 8.2.: To identify the optimal aspect ratio for plasmon sensing, various 'figure of merits' (FOM) are calculated. (a) The classical FOM defined as plasmon sensitivity $S$ divided by plasmon linewidth $\Gamma$ (left axis) is compared to a generalized $\mathrm{FOM}^{*}$ defined as the maximal relative intensity change $|\Delta I / I|$ for a small refractive index change $\Delta n$ (right axis). While the classical FOM has a maximum for an aspect ratio of $\mathrm{AR}=4.3$ and a second maximum at $\mathrm{AR}=3.2$, the generalized $\mathrm{FOM}^{*}$ has its maximum at $\mathrm{AR}=4.2$ with its second local maximum at $\mathrm{AR}=3.1$. (b) The $\mathrm{FOM}_{\text {layer }}^{*}$ takes into account the sensing volume by considering the plasmon change induced by a small layer around the particle normalized to the layer thickness $(0.01 \mathrm{~nm}<l<10 \mathrm{~nm}$ in $1 \mathrm{~nm}$ steps). The plasmon sensing quality $\mathrm{FOM}_{\text {layer }}^{*}$ decreases with increasing layer thickness (inset) and shows a maximal value of $\mathrm{FOM}_{\text {layer }}^{*}=0.55$ for $d \rightarrow 0$ at an aspect ratio $\mathrm{AR}=3.0$. (Data for a are calculated with the BEM for gold nanorods with spherical end-caps and $20 \mathrm{~nm}$ diameter, data for $\mathbf{b}$ within the QSA for spheroids.)

mation: The polarizability $\alpha$ of a spheroid within the QSA is given by [93]:

$$
\alpha=V \varepsilon_{0} \frac{\varepsilon_{r}-1}{1+L\left(\varepsilon_{r}-1\right)}
$$

where, $V$ is the particle volume, $\varepsilon_{r}=\varepsilon_{\text {particle }} / \varepsilon_{\text {medium }}$ is the relative dielectric function of the particle with respect to the medium, and $L$ is a shape factor. The exact equation for $L$ is given by [148]:

$$
L=\frac{1}{\mathrm{AR}^{2}-1}\left\{\frac{\mathrm{AR}}{2\left(\mathrm{AR}^{2}-1\right)^{1 / 2}} \ln \left[\frac{\mathrm{AR}+\left(\mathrm{AR}^{2}-1\right)^{1 / 2}}{\mathrm{AR}-\left(\mathrm{AR}^{2}-1\right)^{1 / 2}}\right]-1\right\}
$$

For $\mathrm{AR}<8$, this formula is simplified to $L \approx(1+\mathrm{AR})^{-1.6}$ with an error of $<5 \%$ [42]. Equating the real part of the denominator of Eq. (8.3) to zero and using the Drude 
Table 8.1.: Summary of the different quantities describing the quality of plasmon sensors regarding their ability to detect changes in their environment: sensitivity $S_{\lambda}$ and $S_{E}$ in wavelength and energy units, the figures of merit as classical definition (FOM), in generalized form $\left(\mathrm{FOM}^{*}\right)$, and for thin layers $\left(\mathrm{FOM}_{\text {layer }}^{*}\right)$.

\begin{tabular}{cccc}
\hline Quality & Definition & $\mathrm{AR}_{\text {opt }}$ & Max value \\
\hline LC 12-1 & 634 & $57 \pm 6$ & $28 \pm 4$ \\
S702 & 700 & $49 \pm 4$ & $18 \pm 2$ \\
S740 & 743 & $55 \pm 6$ & $18 \pm 3$ \\
\hline
\end{tabular}

The last columns list the optimal aspect ratio $\mathrm{AR}_{\mathrm{opt}}$ (the value in bracket corresponds to the second maximum) for gold nanorods with $20 \mathrm{~nm}$ diameter in an aqueous environment and the value of the corresponding quantity at the maximum (see Sec. 8.3 and Sec. 8.4).
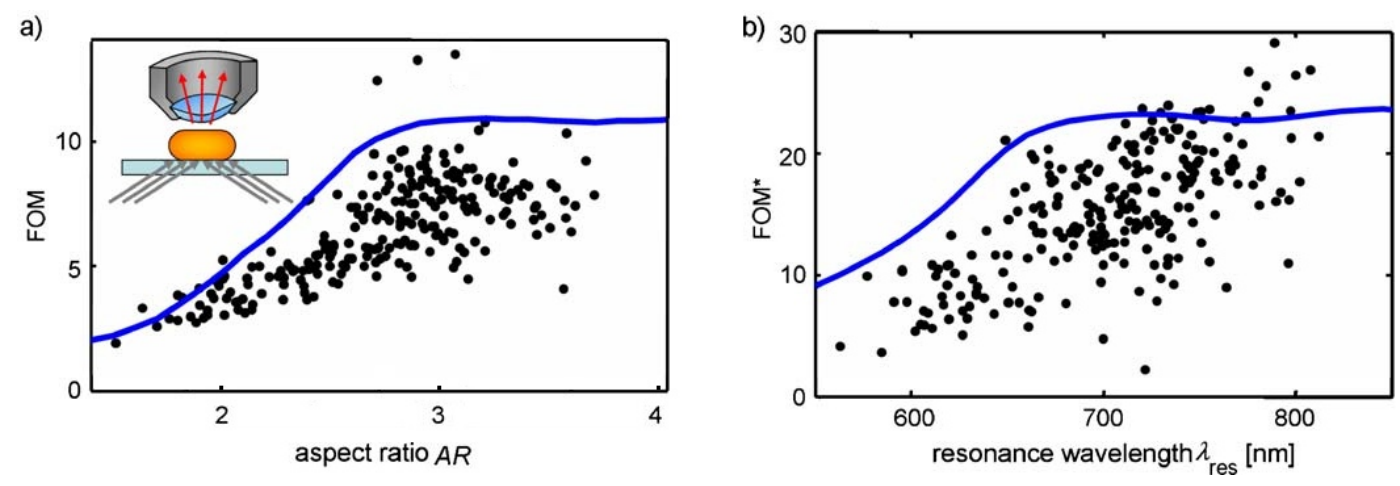

Figure 8.3.: Experimental data (each black dot represents one single particle) for the figures of merit FOM (a) and $\mathrm{FOM}^{*}$ (b) obtained by single particle spectroscopy in a dark field microscope (inset). The absolute values are slightly lower than the theoretical prediction (blue line, see also Fig. 8.2 a) due to the attachment of the gold rods to a glass substrate which limits the accessible surface (inset)

model to describe the dielectric function of the particle $\left(\mathcal{R} e(\varepsilon) \approx \varepsilon_{\infty}-\omega_{p}^{2} / \omega[42]\right)$, the resonance position $E_{\text {res }}$, the plasmon sensitivity $S_{E}$, and the aspect ratio with the highest $S_{E}$ value $\mathrm{AR}_{\max }$ follow: 


$$
\begin{aligned}
E_{\mathrm{res}} & =\frac{\omega_{p}}{\sqrt{n_{b}^{2} / L+\varepsilon_{\infty}-n_{b}^{2}}}, \\
S_{E} & =\frac{d}{d n_{b}} E_{\mathrm{res}}=\frac{\omega_{p} n_{b}(1 / L-1)}{\left(n_{b}^{2} / L+\varepsilon_{\infty}-n_{b}^{2}\right)^{3 / 2}}, \\
\mathrm{AR}_{\max } & =\left(\frac{n_{b}^{2}}{n_{b}^{2}+2 \varepsilon_{\infty}}\right)^{-1 / 1.6}-1 .
\end{aligned}
$$

where, $\omega_{p}$ and $\varepsilon_{\infty}$ are the plasma frequency of the conduction electrons (9 eV for gold [149]) and the polarizability of the inner electrons (9.84 for gold [150]), respectively, and $n_{b}=\sqrt{\varepsilon_{\text {medium }}}$ the medium's refractive index.

The agreement of these simple calculations with the BEM calculations is surprisingly good (Fig. 8.1 black lines) since the quasistatic approximation is only valid for spheroidally shaped particles much smaller than the wavelength of the incident light. The particles we investigate are neither spheroids nor much smaller than the light wavelength (only a factor of up to 5). Our BEM calculations using spherically capped cylinders model the real particle shape more realistically and correctly take the particles' absolute size into account.

Interestingly, the aspect ratio with the highest plasmon sensitivity in energy units $\mathrm{AR}_{\text {max }}$ depends on the polarizability of the inner electrons of the metal $\varepsilon_{\infty}$ and the refractive index of the medium. For water $\left(n_{b}=1.33\right)$ as a medium, the optimal aspect ratio becomes $\mathrm{AR}_{\max }=3.8$. Since the Drude model does not include the contribution of interband damping to the imaginary part of the dielectric function, we conclude that the maximum in plasmonic sensitivity $S_{E}$ is not caused by the effect of those interband excitations.

Yet, what is the reason for the 'optimal aspect ratio' for the three 'figures of merit'? We believe the quality factor $Q$ is the key quantity in explaining this behavior. The quality factor of an oscillation describes the number of oscillations until the oscillation is damped. We find it plausible that a longer plasmon oscillation lifetime (i.e., a higher quality factor) results in a more sensitive dependency on changes in the surroundings. For a classical-driven harmonic oscillator, the resonance frequency 
divided by the linewidth of the resonance $\Gamma$ gives the quality factor $\left(Q=E_{\text {res }} / \Gamma\right.$. Figure 8.4 shows the quality factor as a function of the aspect ratio for gold nanorods (calculated by the BEM and measured experimentally on single particles). Evidently, a maximum is found around $\mathrm{AR}=3$. The initial rise from $Q(\mathrm{AR}=1)=8$ to $Q(\mathrm{AR}=3)=22$ is easily explained considering the reduced plasmon damping as the resonance shifts to lower energies with an increasing aspect ratio [135]. The slow decrease above the aspect ratio of 3 is due to the decreasing resonance frequency divided by the (more or less) constant Drude damping.

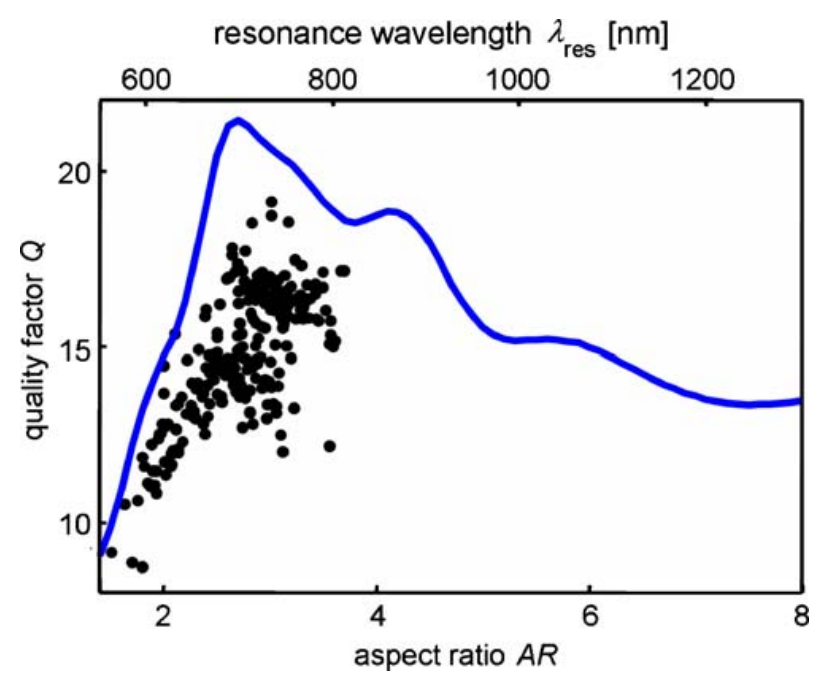

Figure 8.4.: The quality factor $Q$ (determined via $Q=E_{\mathrm{res}} / \Gamma$ ) shows a maximum for rods with an aspect ratio of approximately $\mathrm{AR}=3$ and decreases for larger aspect ratios. The blue line represents the results obtained by BEM calculations, while the black dots correspond to measurements on single gold nanorods.

The similarity between the way the quality factor and the figures of merit depend on the aspect ratio is a strong hint but not solid proof that one is the cause of the other. It is desirable to have more detailed theoretical investigations of this aspect.

\subsection{Conclusion}

In summary, for the four measures for an 'optimal plasmonic sensor', namely sensitivity $S$, figure of merit FOM, generalized figure of merit $\mathrm{FOM}^{*}$, and figure of merit 
for thin layers $\mathrm{FOM}_{\text {layer }}^{*}$, there is an optimum aspect ratio around 3-4, where gold nanorods show the best sensitivity. Only the plasmon sensitivity in wavelength units $S_{E}$ increases steadily with aspect ratio. Contrary to many claims, the plasmonic properties of gold nanorods do not steadily increase towards the infrared spectral region but show an optimum in the near infrared. Even though gold nanorods show very strong plasmon resonances with high quality factors and are, at the same time, small in volume, we cannot exclude the idea that other shapes of metal structures (bi-pyramids, rattles, pairs of particles, EIT structures, etc) are even better plasmon sensors. We hope that our new definition of a generalized figure of merit and the corresponding generalized figure of merit for small layers will trigger the comparison of plasmon sensor quality of many more complicated metal structures.

\subsubsection{Acknowledgment}

We acknowledge financial support by the DFG through the Emmy Noether Program (SO712/1 - 3), the MAINZ graduate school of excellence, and the Graz Advanced School of Science (NAWI GASS).

\subsection{Appendix - Methods}

\subsubsection{Simulations based on boundary element method}

In our computational approach, we discretize the surface of the nanoparticle by a set of triangles and match the electromagnetic potentials at the triangle centers. By fulfilling the boundary conditions imposed by Maxwell's equations through auxiliary surface charges and currents, we end up with a generic and flexible scheme that allows us to compute the optical properties of arbitrarily shaped nanoparticles with complex geometry embedded in dielectric environments.

The dielectric function of the gold nanorods was extracted from optical data [41] and the chosen mesh sizes allow for a spatial resolution of approximately $1 \mathrm{~nm}$. 


\subsubsection{Determination of $S$, FOM, FOM $^{*}$, and FOM $_{\text {layer }}^{*}$}

We calculated the light scattering cross-section $C_{\text {sca }}$ for every particle shape (diameter $20 \mathrm{~nm}$, length from $20 \mathrm{~nm}$ to $160 \mathrm{~nm}$ in steps of $2 \mathrm{~nm}$ ) in $n_{b_{1}}=1.33$ and in $n_{b_{2}}=1.34$ as a function of wavelengths $\lambda$ (in steps of $1 \mathrm{~nm}$ ).

The $\mathrm{FOM}^{*}$ was calculated according to the following equation:

$$
\mathrm{FOM}^{*}=\left[\frac{\left|C_{\mathrm{sca}_{1}}(\lambda)-C_{\mathrm{sca}_{2}}(\lambda)\right|}{1 / 2\left(C_{\mathrm{sca}_{1}}(\lambda)+C_{\mathrm{sca}_{2}}(\lambda)\right)}\right]_{\max } /\left(n_{b_{2}}-n_{b_{1}}\right)
$$

The $\mathrm{FOM}_{\text {layer }}^{*}$ was calculated in the same way in the quasistatic approximation for spheroids for layer thicknesses $l=0.01 \mathrm{~nm}$ to $10 \mathrm{~nm}$ in steps of $1 \mathrm{~nm}(0.01 \mathrm{~nm}$ until the first nanometer).

\subsubsection{Gold nanorods preparation}

Gold nanorods were synthesized according to the seeded growth procedure published by Nikoobakht et al. [151]. In this two-step synthesis, preformed seeds grow into rods in a concentrated surfactant solution. The samples used in this work were characterized by ensemble extinction spectroscopy and transmission electron microscopy to obtain the mean diameters and lengths of the different samples. The samples we used had the following properties:

\begin{tabular}{cccc}
\hline Name & $\lambda_{\text {res }}$ & Width & Length \\
LC 12-1 & 634 & $57 \pm 6$ & $28 \pm 4$ \\
S702 & 700 & $49 \pm 4$ & $18 \pm 2$ \\
S740 & 743 & $55 \pm 6$ & $18 \pm 3$ \\
\hline
\end{tabular}

\subsubsection{Single particle spectroscopy}

To investigate the spectral shift by changes in the refractive index of the environment on the single particle level, we dilute the nanorod suspensions 1:100 with distilled water and rinse them for 5 min through a flat glass capillary connected to PET tubing. Subsequent rinsing of $1 \mathrm{M}$ sodium chloride solution for $1 \mathrm{~min}$ can increase 
the number of immobilized particles. Afterwards, the glass capillary is rinsed for at least 15 min with distilled water $\left(n_{b}=1.333\right)$ to remove as many of the molecules as possible that were attached to the particle surface, and the scattering spectra of all particles in the field of view recorded. After rinsing the glass capillary with glucose solution $\left(25 \mathrm{wt} . \% n_{b}=1.372\right)$ for $15 \mathrm{~min}$, we again investigate the scattering spectra of the same particles and determine the quantities listed in Table 8.1. 



\title{
9. PAPER: Highly sensitive plasmonic silver nanorods
}

\author{
Arpad Jakab ${ }^{1}$, Yuriy Khalavka ${ }^{1}$, Jan Becker ${ }^{1}$, Andreas Trügler ${ }^{2}$, Christina \\ Rosman $^{1}$, \\ Ulrich Hohenester ${ }^{2}$, and Carsten Sönnichsen ${ }^{1}$ \\ ${ }^{1}$ MAINZ graduate school of excellence and Institute of Physical Chemistry, University of Mainz, \\ Jakob-Welder-Weg 11, 55128 Mainz, Germany \\ ${ }^{2}$ Institut für Physik, Karl-Franzens-Universität Graz,Universitätsplatz 5, 8010 Graz, Austria
}

submitted 2011

\begin{abstract}
We report experimental evidence of increased single particle plasmonic sensitivity of silver nanorods compared to gold rods with similar aspect ratios. We measured the plasmonic sensitivity of single gold and silver nanorods by monitoring the spectroscopic change on changing the environment from water to $12.5 \%$ sugar solution. We find that silver has 1.2 to 2 times larger sensitivity than gold, in good agreement with simulations based on the Boundary Element Method (BEM). Using the Drudemodel of optical properties of metals together with the quasi-static-approximation for localized surface plasmons we show that the reason for the higher sensitivity of silver is in the lower background polarizability of the inner shell electrons. However, the reversibility of the silver nanorod sensors, upon repeated cycles of environment changes, is much worse than of gold nanorods. Blocking parts of the illumination light by inserting a long-pass filter reduces this problem significantly.
\end{abstract}




\subsection{Introduction}

Sensor devices based on the refractive index sensitivity of surface plasmons have emerged on the market in the last decade. Attempts to increase the sensitivity and reduce the sensor size focus on single particle based sensors and localized surface plasmons polaritons or particle plasmons $[138,152,153]$. As recently reported, single particle sensors can have a better sensitivity than propagating surface plasmon based sensors [154].

The spectral position of the localized surface plasmon is highly dependent on the dielectric properties of the surrounding medium [155]. For example, a change of the surrounding refractive index of 0.02 from 1.333 to 1.353 results in a red shift of the resonance wavelength by $5.1 \mathrm{~nm}$ for a gold nanorod with an aspect ratio of 2.5. For optimal sensing of smallest dielectric changes (i.e. binding of single molecules), the spectral shift of the resonance wavelength upon changing the surrounding medium has to be maximized. Increasing the spectral shift can be achieved by various methods like: optimizing the shape and size [140,156-158], inter-particle distance [124,159] or the material of the particle $[158,160]$. In recent times, efforts have been intensified to investigate silver nanoparticle sensitivity $[161,162]$. In this work, we present experimental results of single silver nanorod sensitivity revealing that silver nanorods have a larger sensitivity than gold nanorods at the same resonance wavelength and that - contrary to earlier predictions [139] - the background polarizability of the bound electrons strongly influences the sensitivity.

\subsection{Synthesis}

For our study, we form silver nanorods with various aspect ratios in solution adapting a two-step "seeded growth" approach developed by Kitaev et al. [163] with small modifications in illumination and heating. In the first step, faceted silver "seeds" are produced under light illumination. We use a blue LED lamp (141 mW, peak maximum at $459.5 \mathrm{~nm}$, FWHM $20.53 \mathrm{~nm}$ ) instead of the high power metal halide lamp used by Kitaev and co-workers. In the second step, the seeds are added to a "growth solution" where they develop into silver nanorods. We preheat the growth solution in a consumer microwave at $90 \mathrm{~W}$ for 1 minute. Then $\mathrm{AgNO}_{3}$ is added 
and the solution is placed into the microwave again for heating between 2 and 6 minutes resulting in rods with increasing aspect ratio (AR). Two minutes of heating result in rods with an aspect ratio of $2.4 \pm 0.8$, four minutes yield an aspect ratio of $2.9 \pm 1.3$, and six minutes an aspect ratio of $4.0 \pm 1.6$. (Fig. 9.1, top). We compare the plasmonic sensitivity of those silver rods with gold nanorods produced by the method of Nikoobakht et al. [151].

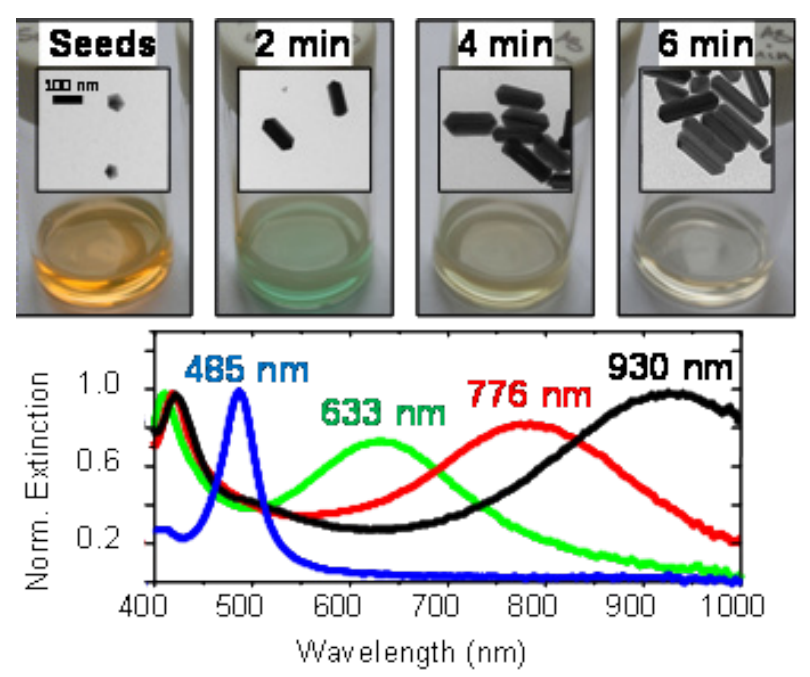

Figure 9.1.: Representative silver nanorod samples are shown as photographs and TEM images together with the corresponding ensemble extinction spectra (bottom). The samples correspond to: Ag-seeds and Ag-nanorods grown with 2, 4, and 6 minutes of heating time (from left to right). Their plasmon peak increases from $485 \mathrm{~nm}$ to $633 \mathrm{~nm}, 776 \mathrm{~nm}$, and $930 \mathrm{~nm}$.

\subsection{Plasmonic nanorod sensitivity}

We observe the light scattering spectra of the single nanoparticles with a scanning dark-field setup [131] that allows for a rapid measurement of a large number of immobilized nanoparticles (Fig. 9.2 A). To measure the plasmon resonance shift upon changes in the refractive index of the environment (single particle sensitivity), the nanoparticles are immobilized in a flat glass capillary or flow cell (the flow-cell preparation is described in detail in the Methods section). 


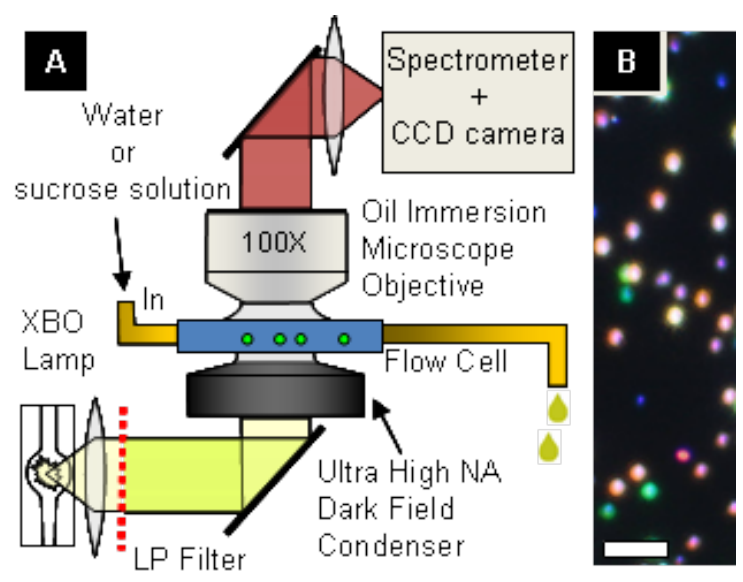

Figure 9.2.: (A) Experimental wet environment dark field setup designed for single nanoparticle spectroscopy in various surrounding media. (B) Typical dark-field image of the nanoparticles, the scale bar is $10 \mu \mathrm{m}$.

To immobilize nanoparticles in the flow cell, we add a suspension of nanoparticles in water followed by a salt solution. The concentration of nanoparticles is adjusted by dilution to create a nanoparticle density in the field-of-view that allows the distinction of single particles (Fig. 9.2 B); i.e. an average inter-particle distance of about $10 \mu \mathrm{m}$. The addition of salt screens the surface charges that stabilize the nanoparticle suspension which leads to nonspecific adsorption of nanoparticles to the glass surface of the flow cell. For gold nanorods, we used $0.1 \mathrm{M} \mathrm{NaCl}$, for silver $0.1 \mathrm{M}$ $\mathrm{KNO}_{3}$ because, as discussed later, chloride ions can cause structural changes to silver nanoparticles.

Table 9.1 summarizes the size characteristics of the nanorods used for single particle spectroscopic measurements. We used only silver nanorods from samples with mean longitudinal plasmon resonances of $633 \mathrm{~nm}$ and $776 \mathrm{~nm}$ (green and red curves in Fig. 9.1). Those silver nanorods had a mean width of about 55-60 nm and aspect ratios of 2.4:1 and 2.9:1, respectively. In comparison, the gold nanorods were smaller with a width of 11-28 nm but with comparable aspect ratios. More details about the nanoparticle size distribution are presented in Fig. 9.5 and Table 9.2 in the Supporting Information 9.9 . 


\begin{tabular}{cccc}
\hline Nanorod Material & $\begin{array}{c}\text { Longitudinal } \\
\text { ensemble peak }\end{array}$ & Width & $\begin{array}{c}\text { Aspect Ratio } \\
\text { (length/width) }\end{array}$ \\
\hline $\mathrm{Ag}$ & $633 \mathrm{~nm}$ & $55 \pm 10 \mathrm{~nm}$ & $2.4 \pm 0.8$ \\
$\mathrm{Ag}$ & $776 \mathrm{~nm}$ & $59 \pm 14 \mathrm{~nm}$ & $2.9 \pm 1.3$ \\
$\mathrm{Au}$ & $634 \mathrm{~nm}$ & $28 \pm 4 \mathrm{~nm}$ & $2.0 \pm 0.1$ \\
$\mathrm{Au}$ & $702 \mathrm{~nm}$ & $18 \pm 2 \mathrm{~nm}$ & $2.7 \pm 0.1$ \\
$\mathrm{Au}$ & $743 \mathrm{~nm}$ & $18 \pm 3 \mathrm{~nm}$ & $3.1 \pm 0.2$ \\
$\mathrm{Au}$ & $816 \mathrm{~nm}$ & $11 \pm 2 \mathrm{~nm}$ & $4.1 \pm 0.1$ \\
\hline
\end{tabular}

Table 9.1.: Properties of the silver and gold nanorods used in our study (sizes from TEM images).

\subsection{Sensitivity measurements}

The plasmon sensitivity quantifies the dependence of the plasmon resonance wavelength or frequency on changes in the dielectric properties of the surrounding medium. Sensitivity $S$ (in wavelength units) is therefore defined by $S=d \lambda_{\text {res }} / d n_{b}$, where $\lambda_{\text {res }}$ is the plasmon resonance wavelength and $n_{b}$ is the refractive index of the surrounding medium. There are more elaborate measures to quantify the sensor quality of nanoparticles taking into account more realistic sensor concepts, for example FOM [144,164], FOM*, [156] MFOM [165]. All of these quantities depend on the plasmon sensitivity. Here we focus on the sensitivity in wavelength units and refer to this term simply as 'sensitivity'.

We determined the plasmonic sensitivity experimentally by recording spectra of single immobilized nanoparticles in the initial environment (pure water, $n_{1}=1.3330$ ), then immersed in $12.5 \%$ sucrose solution $\left(n_{2}=1.3454\right)$, and at the end again in water. The resulting experimental results are shown as red crosses (gold nanorods) and blue stars (silver nanorods) in Fig. 9.3 (top panel). The red and blue continuous lines are trend-lines of the datasets. The experimentally obtained trend-lines qualitatively follow the trend simulated with the BEM [83] (dashed lines) but are lower than the simulation. However, the simulation was performed with a homogenous medium around the particles whereas in the experiment, the particles are attached to a glass substrate reducing the influence of the change in medium. The fact that the experimental silver trend shows a stronger deviation from simulations than the gold nanorods might be caused by additional damping of the plasmon resonance due to their penta-twinned crystal structure [166]. 


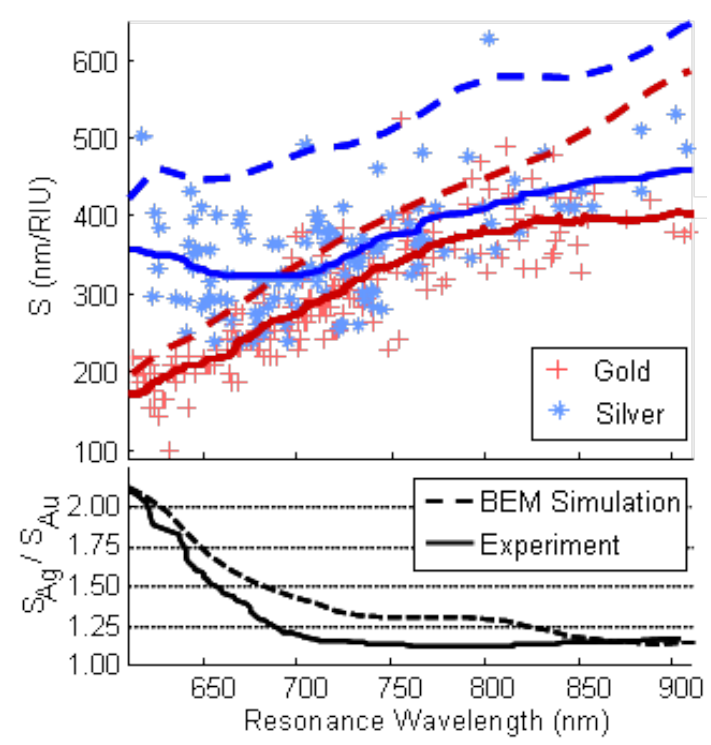

Figure 9.3.: Top: Single particle sensitivity data plotted for each particle both for silver and gold. The continuous blue and red lines are the averages of the experimental data of silver and gold, respectively, the dashed lines are obtained in BEM simulations. Bottom: the experimentally obtained (continuous line) and BEM-simulated (dashed line) sensitivity enhancement $\left(S_{\mathrm{Ag}} / S_{\mathrm{Au}}\right)$ of silver nanorods in relation to gold nanorods.

The average silver sensitivity is larger than the gold sensitivity. The sensitivity of silver nanorods reaches values above $400 \mathrm{~nm} /$ RIU that is, up to now, the largest value for rod-like objects in this wavelength range [157]. Especially in the wavelength range of 600 to $700 \mathrm{~nm}$, where gold has only a sensitivity of $150-270 \mathrm{~nm} / \mathrm{RIU}$, silver performs better with sensitivities of above $300 \mathrm{~nm} / \mathrm{RIU}$. We believe that the scattering of the data points around the trend lines is primarily caused by the varying thicknesses of the surface stabilizing layer (Ag: PVP, Au: CTAB) around the particles.

To directly compare the sensitivity of gold and silver, we show the ratio of their sensitivity (the sensitivity enhancement of silver compared to gold $S_{\mathrm{Ag}} / S_{\mathrm{Au}}$ ) in the bottom plot of Fig. 9.3, both for the experimental data and the simulation. The experiment and simulation agree well in this diagram, the substrate effect effectively cancels out. At shorter wavelengths, around $610 \mathrm{~nm}$, the enhancement reaches a factor of two, at longer wavelengths the enhancement drops to a value between 1.1 and 1.2 . 


\subsection{Discussion and Model}

To understand the origin of the higher plasmonic sensitivity of silver nanorods compared to gold nanorods, it is sufficient to use the quasi-static approximation to calculate the scattering cross section together with a simple Drude-type model for the dielectric function for gold and silver. Within those two approximations, the sensitivity $S$ at a given resonance wavelength $\lambda$ is given by the following equation (see Supporting Information 9.9 for details):

$$
S(\lambda)=\frac{\lambda}{n_{b}}\left(1-\frac{\lambda_{p}^{2}}{\lambda^{2}} n_{\infty}^{2}\right)
$$

where $\lambda_{p}=2 \pi c / \omega_{p}$ is the bulk plasmon wavelength $\left(\lambda_{p}=136 \mathrm{~nm}\right.$ for gold and silver), $n_{\infty}=\sqrt{\varepsilon_{\infty}}$ is the background refractive index, the square root of the background dielectric constant, originating from the polarizability of the bound electrons $\left(\varepsilon_{\infty, \mathrm{Au}}=9.84\right.$ for gold and $\varepsilon_{\infty, \mathrm{Ag}}=3.7$ for silver correspond to $n_{\infty, \mathrm{Au}}=3.1$ and $n_{\infty, \mathrm{Ag}}=1.9$, respectively), and $n_{b}$ is the refractive index of the surrounding medium. The equation above reveals that, contrary to earlier reports [139], the sensitivity depends on the material through the strong difference in the background polarizability $\varepsilon_{\infty}$ of gold and silver (the sensitivity decreases with increasing $\varepsilon_{\infty}$ or $n_{\infty}$ ). Despite the simplifications of the quasi-static approach and the Drude model for the dielectric function, the equation above predicts the sensitivity of nanorods remarkably well (Fig. 9.6 in the Supporting Information compares the sensitivity calculated with the BEM with tabulated dielectric values for gold and silver, to the sensitivity calculated with the simple model above). We have not used any assumptions about the physical dimensions and shape of the particle during the derivation of the equation above. The sensitivity of a plasmonic nanoparticle at a given wavelength is therefore independent from its shape and size as long the conditions for the approximations are valid (e.g. no retardation effects, no interband damping). 


\subsection{Sensing Reversibility}

Whereas sensitivity alone seems to favor silver over gold, another important aspect for sensors is the sensing reversibility upon repeated changes of the environment. Given the fact that silver is less noble than gold, we carefully checked the reversibility. For this purpose, our measurements always went back to the initial environment at the end and we recorded the difference in resonance wavelength between initial and final resonance wavelength, a quantity we call the sensing cycle shift. For a reliable sensor, the mean sensing cycle shift should remain close to the experimental uncertainty $( \pm 0.5 \mathrm{~nm})$ and we had disregarded all particles that did not show a full reversibility in the datasets for estimating particle sensitivity (within one standard deviation). Halide ions $\left(\mathrm{Cl}^{-}\right.$and especially $\left.\mathrm{Br}^{-}\right)$induce a reshaping of silver nanoparticles above a threshold concentration of $\sim 10^{-8} \mathrm{M}$ so we took care to avoid any use of those ions $[167,168]$. Generally, we found that even without halide ions present, silver nanoparticles showed much worse reversibility performance than gold. We suspected a light induced process so we measured the reversibility with different filters in the light path.

In Fig. 9.4 we show the sensing cycle shift of single nanorods plotted as a cumulative distribution probability (an integrated histogram that allows an easy visualization of the mean value) measured under different illumination conditions where each dot corresponds to the sensitivity cycle shift of a single particle. The sensing cycle shift is decreasing with increasing cut-off wavelength of the long pass filter. The mechanism of this light-induced irreversible shift remains unclear and is probably a combination of heating and photochemical processes. The shift has nothing to do with changing the environment as we checked in experiments that show a red shift of nanoparticles monitored continuously in water (Fig. 9.9 in the Supporting Information 9.9). Our experiments indicate that a sufficiently high cut-off filter allows the use of silver nanoparticles for sensing in environments without halide ions.

\subsection{Conclusion}

We have presented experimental results of silver nanorod sensitivity and compared them to results found for gold nanorods. Silver nanorods have up to two times 


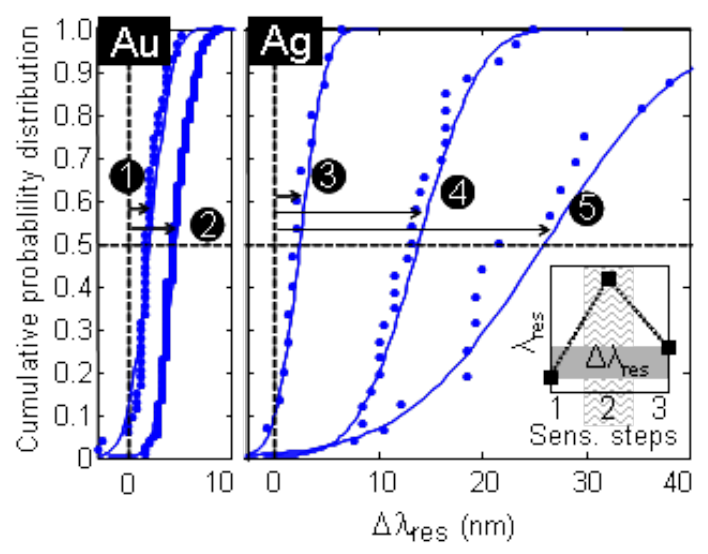

Figure 9.4.: Cumulative distribution of single nanoparticle resonance wavelength shifts after one sensing measurement cycle (the sensing cycle shift). The continuous lines are Gaussian fits, the arrow indicates the mean cycle shift. Left graph: (1) $\mathrm{Au}$ rods with $610 \mathrm{~nm}$ long pass (LP) filter (2) Au rods without LP filter. Right graph: (3) Ag rods with $610 \mathrm{~nm}$ LP filter, (4) Ag rods with $530 \mathrm{~nm}$ LP filter (5) $\mathrm{Ag}$ rods without LP filter. Inset at bottom right: definition of sensing cycle shift $\Delta \lambda_{\text {res }}$ in the cycle $n_{1} \rightarrow n_{2} \rightarrow n_{1}$.

larger sensitivity than gold nanorods in the spectral range of 600 to $700 \mathrm{~nm}$. In the region of 700 to $900 \mathrm{~nm}$, the sensitivity is still about 10 to $20 \%$ higher. From simple theoretical arguments (Drude's model of optical properties of metals and the quasi-static-approximation), we could identify the lower background polarizability of silver compared to gold as the main reason. The reversibility of silver nanorod based sensors is, generally, worse than gold nanorod based sensors, but adding a $610 \mathrm{~nm}$ cut-off long-pass filter in the light path, resulted in a reasonably reversible sensor for both cases.

\subsection{Methods}

\subsubsection{Reagents}

Silver nitrate (99\%), sodium citrate tribasic dihydrate (Aldrich 99\%), polyvinylpyrrolidone (PVP, Mw 40K), sodium borohydride (99\%, Aldrich), L-arginine (98\%, Aldrich) were used as received. Millipore deionized water was used as solvent. 


\subsubsection{Synthesis of Silver Nanorods}

Silver nanorods were synthesized following mainly the procedure developed by Kitaev et al. [163]. Decahedral Ag nanoparticles were used as seeds. For their synthesis, a precursor solution of silver nanoparticles was first prepared using a mixture of 1.000 $\mathrm{mL}$ of $0.05 \mathrm{M}$ sodium citrate, $0.060 \mathrm{~mL}$ of $0.05 \mathrm{M}$ PVP, $0.100 \mathrm{~mL}$ of $0.005 \mathrm{M} \mathrm{L}$ arginine, $0.400 \mathrm{~mL}$ of $0.005 \mathrm{M} \mathrm{AgNO}_{3}$ and $14.0 \mathrm{~mL}$ of deionized water in a $20 \mathrm{~mL}$ vial and then reduced by adding $0.160 \mathrm{~mL}$ of $0.10 \mathrm{M} \mathrm{NaBH}_{4}$. The resulting yellow solution was stirred for several minutes. To produce decahedra, the blue diode array lamp with $\lambda_{\max } 460 \mathrm{~nm}$ with an exposure time of 10 hours was used (spectrum in the Supporting Information 9.9). In the next step, $2 \mathrm{~mL}$ of the freshly prepared decahedral Ag seed solution was centrifuged to replace the supernatant with $1.0 \mathrm{~mL}$ of pure deionized water.

Having prepared the decahedra seed solution, $1 \mathrm{~mL}$ of the purified decahedra solution, $2.0 \mathrm{~mL}$ of water, $0.400 \mathrm{~mL}$ of $0.05 \mathrm{M}$ sodium citrate, and $0.066 \mathrm{~mL}$ of $0.05 \mathrm{M}$ PVP were heated to $95{ }^{\circ} \mathrm{C}$ in a $20 \mathrm{~mL}$ vial in microwave oven at $90 \mathrm{~W}$ power. After temperature equilibration, $0.400 \mathrm{~mL}$ of silver nitrate $(0.005 \mathrm{M})$ were added at once to produce rods with an aspect ratio up to 4-5 in high yield in 2-6 minutes of the reaction under $90 \mathrm{~W}$ microwave power.

\subsubsection{Characterization}

Transmission electron microscopy was performed using FEI Tecnai F30 and Phillips EM-420 microscopes. Nanoparticle solutions were deposited on a carbon-coated Formvar grid (Plano). Operating voltage was varied from 120-300 kV. The average size and standard deviation were determined from TEM images by averaging diameters of about 100 particles. Ensemble optical spectra were acquired with Ocean Optics USB 2000 spectrometer.

\section{Single Particle Microscopy}

The single particle spectroscopy was performed with a self-build microscopy setup employing a 2D piezo scanner. The setup shown in Fig. 9.2 consists of a positioning stage (PI P-542.2CD Nanopositioning and Scanning Stage), microscope optics, an imaging spectrometer (Princeton Instruments/ACTON SpectraPro 2300), and a 
CCD camera (Princeton Instruments Pixis 400B). During a spectral scan, the sample is moved in steps of $1 \mu \mathrm{m}$ along the x-axis. At each step, the spectrum of the particles in the center column is taken. The scan window width is adjusted to the step size to ensure gapless coverage of the scanned area.

\section{Flow Cell}

The glass capillary (Vitrotube W5010-100 by Vitrocom) is initially UV-irradiated for 15 minutes and left for another 15 minutes in the reactive atmosphere, then we attach thin polyethylene tubing using epoxy glue to construct the flow cell system. The flow cell is purged with $1 \%$ Helmanex solution for 24 hours, and finally cleaned with hot deionized water for 1 hour.

\subsection{Supporting Material}
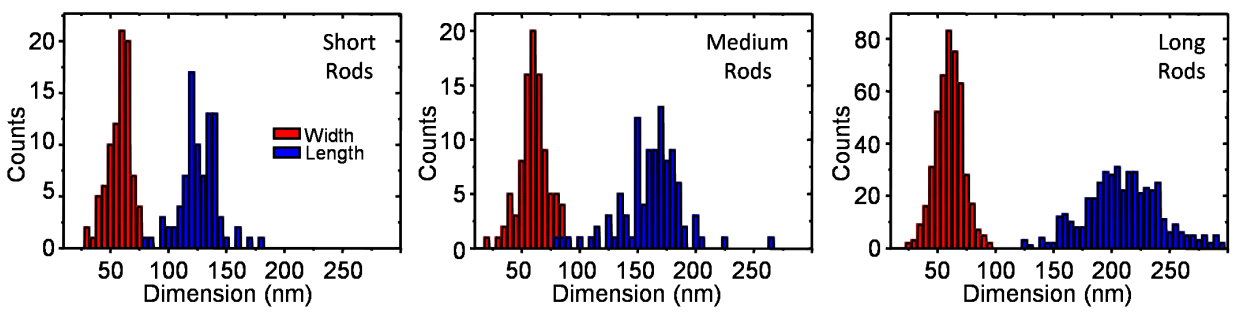

Figure 9.5.: Histogram of width (red) and length (blue) for Ag-rod samples width different average length (from TEM images).

\begin{tabular}{cccccc}
\hline Sample & No. of Part. & Width $(\mathrm{nm})$ & STD $(\mathrm{nm})$ & Length $(\mathrm{nm})$ & STD $(\mathrm{nm})$ \\
\hline Short Rods & 88 & 55 & 10 & 124 & 16 \\
Med. Rods & 98 & 59 & 13 & 159 & 27 \\
Large Rods & 459 & 58 & 12 & 212 & 39 \\
\hline
\end{tabular}

Table 9.2.: Dimensional characterization of the silver nanoparticles that were used for single particle sensitivity measurements. The relative standard deviation of the widths is slightly larger (20\%) than of the lengths (15\%). 


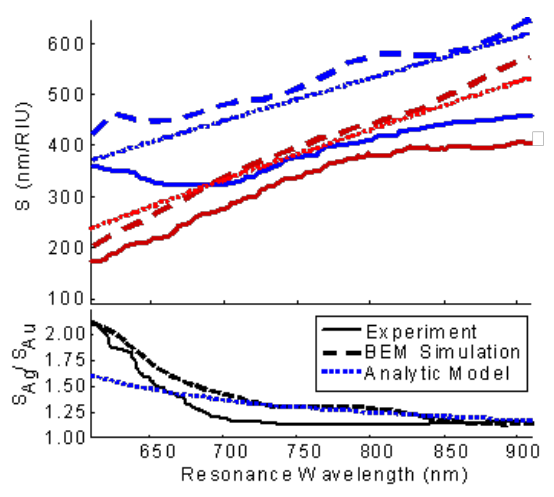

Figure 9.6.: Comparison of sensitivities obtained by BEM simulation (dashed lines) and the free electron Drude-Model of Eq. (9.7) below (dotted lines). For reference, we have also included the experimentally obtained sensitivity data (continuous lines). Despite the simplifications used in the free electron DrudeModel, it follows the simulated curve remarkably well.

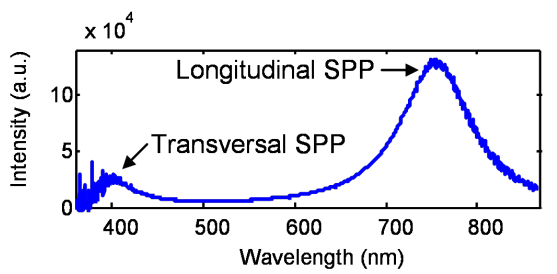

Figure 9.7.: Example of a white light scattering spectrum of a single silver nanorod. For unpolarized excitation, both the long axis (or longitudinal) and the short axis (or transversal) plasmon resonances are visible.

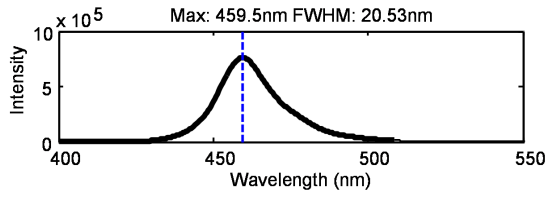

Figure 9.8.: The spectrum of the blue LED lamp used to grow silver seeds.

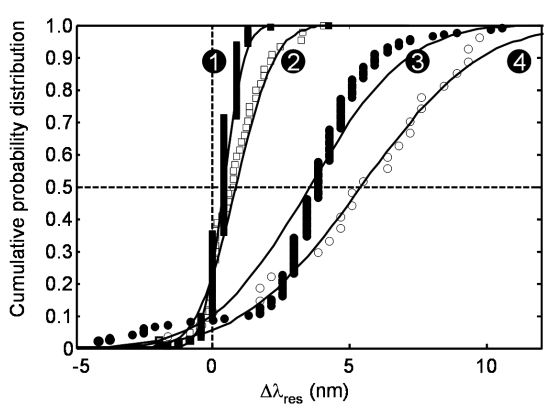

Figure 9.9.: Spectral stability of nanoparticles measured under identical conditions as during sensitivity measurements, except that here the surrounding medium was always water. The graph shows the influence of the long pass filter on the spectral stability of $\mathrm{Au}$ and $\mathrm{Ag}$ nanoparticles by plotting the cumulative probability (integrated histogram) of the spectral shift $\Delta \lambda_{\text {res }} 1$ : $\mathrm{Au}$ with $610 \mathrm{~nm}$ long pass filter, 2: $\mathrm{Ag}$ with $610 \mathrm{~nm}$ long pass filter, 3: Au without long pass filter, 4: Ag without long pass filter. The spectral stability of gold is better (smaller average shift) than of silver. We assume that some activation energy related photochemical process is inducing an irreversible reshaping of the nanoparticle. 


\subsubsection{Derivation of the plasmonic sensitivity equation}

Within the quasi-static approximation (i.e. for particle dimensions much smaller than the light wavelength), the scattering cross-section of a particle is proportional to the square of the modulus of the polarizability: $C_{\text {sca }} \propto|\alpha|^{2}$. The polarizability is given by:

$$
\alpha=\frac{V}{4 \pi} \frac{\varepsilon_{r}-1}{1+L \varepsilon_{r}-1} .
$$

Where $\varepsilon_{r}=\varepsilon(\omega) / \varepsilon_{r}$ is the relative dielectric function of the particle $\varepsilon(\omega)$ to the embedding medium $\varepsilon_{b}, L$ is a geometric depolarization form factor that is well approximated by $L=(1+\mathrm{AR})^{-1.6}$ for rods with aspect ratio (AR) between 1 to 8 . The plasmon resonance occurs at the frequency where the denominator becomes zero, i.e. $1+L\left(\varepsilon_{r}-1\right)=0$.

Within the Drude model, the real part of the metals dielectric function is given by

$$
\Re e(\varepsilon)=\varepsilon_{\infty}-\frac{\omega_{p}^{2}}{\omega^{2}+\gamma^{2}} \approx \varepsilon_{\infty}-\frac{\omega_{p}^{2}}{\omega^{2}}
$$

where $\gamma$ is the free electron damping, which is negligible for gold and silver in the visible spectral range, $\omega_{p}$ is the plasma frequency ( $\omega_{p}=9.1 \mathrm{eV}$ for both gold and silver), and $\varepsilon_{\infty}$ the background polarizability arising from the bound electrons $\left(\varepsilon_{\infty, \mathrm{Au}}=9.84\right.$ for gold and $\varepsilon_{\infty, \mathrm{Ag}}=3.7$ for silver). The lower value for background polarizability of silver reflects the deeper lying d-band with an offset to the Fermi-energy of $4 \mathrm{eV}$ compared to $2 \mathrm{eV}$ for gold.

Using Eq. (9.3) as the frequency dependent dielectric function for the nanoparticle and inserting it into the resonance condition (denominator in Eq. (9.2) is zero) $1+L\left(\varepsilon_{r}-1\right)=0$, we can obtain the resonance wavelength $\lambda_{\text {res }}=2 \pi c / \omega_{\text {res }}$. We replace $\varepsilon_{b}$ for practical purposes with the refractive index of the surrounding medium $n_{b}$ $=\varepsilon_{b}^{1 / 2}$

$$
\lambda_{\mathrm{res}}=\lambda_{p} \sqrt{\varepsilon_{\infty}+n_{b}^{2}\left(\frac{1}{L}-1\right)},
$$

where $\varepsilon_{p}=2 i c / \omega_{p}=136 \mathrm{~nm}$ is the bulk plasmon wavelength with $c$, the speed of light in vacuum.

Since the sensitivity is defined by the change of the resonance wavelength $\lambda_{\text {res }}$ with 
the surrounding refractive index $n_{b}$, we arrive at the equation for the sensitivity:

$$
S=\frac{d}{d n_{b}} \lambda_{\mathrm{res}}=\frac{\lambda_{p} n}{\sqrt{n_{\infty}^{2}\left(\frac{L}{1-L}\right)^{2}+n_{b}^{2}\left(\frac{L}{1-L}\right)^{2}}},
$$

where $n_{\infty}=\varepsilon_{\infty}^{1 / 2}$ is the background refractive index. To be able to directly compare the experimental data to the results of the analytic expression in Eq. (9.5), we need to express the sensitivity as function of the resonance wavelength. Thus we use Eq. (9.4) and rearrange it to express $L / 1-L$ as function of $n_{\infty}, n_{b}, \lambda_{p}$ and $\lambda_{\text {res }}$ we get:

$$
\left(\frac{L}{1-L}\right)=n_{b}^{2} / \frac{\lambda_{\mathrm{res}}^{2}}{\lambda_{p}^{2}}-n_{\infty}^{2} .
$$

Inserting the right side of Eq. (9.6) into Eq. (9.5) and writing $\lambda$ for $\lambda_{\text {res }}$ we get:

$$
S(\lambda)=\frac{\lambda}{n_{b}}\left(1-\frac{\lambda_{p}^{2}}{\lambda^{2}} n_{\infty}^{2}\right) .
$$

\subsection{Acknowledgments}

We acknowledge financial support by the DFG through the Emmy Noether Program (SO712/1-3) and the Graz Advanced School of Science (NAWI GASS). Arpad Jakab and Yuriy Khalavka were recipients of the MAINZ graduate school of excellence fellowship through funding of the Excellence Initiative (DFG/GSC 266). 


\title{
10. PAPER: Superresolution Moiré Mapping of Particle Plasmon Modes
}

Daniel Koller, Ulrich Hohenester, Andreas Hohenau, Harald Ditlbacher, Frank Reil,

Nicole Galler, Franz Aussenegg, Alfred Leitner, Andreas Trügler, and Joachim

Krenn

Institute of Physics and Erwin Schrödinger Institute for Nanoscale Research, Karl-Franzens-University, 8010 Graz, Austria

Published in: Phys. Rev. Lett. 104, 143901 (2010)

\begin{abstract}
The spontaneous emission rate of a fluorophore provides a direct probe of the photonic local density of states at the fluorophore position. Here we exploit this capability to map the plasmonic modes of gold nanoparticles by imaging the fluorescence intensity in combined regular arrays of identical gold and fluorophore-doped polymer nanoparticles. By varying the distance between gold and polymer particles across the array, the fluorophore emission generates an optical Moiré pattern corresponding to a magnified spatial map of the plasmonic mode, which can be directly imaged with an optical microscope. Our results are corroborated by supplementary theoretical model calculations.
\end{abstract}


Surface plasmons enable the nanoscale localization of light and provide an effective means to control the excitation and emission properties of quantum systems. These appealing properties have triggered a large variety of recent research and development in fields as diverse as data storage [13], quantum optics [16,17], optoelectronics $[18,19]$, photovoltaics [20], sensors [7], and cancer research [6]. With a demand for ever increasing miniaturization and integration densities for many of these applications, the spatially and spectroscopically resolved characterization of plasmonic nanostructures has to be improved as well. Recent developments in this direction include progress in near field scanning microscopy $[169,170]$, two-photon luminescence based imaging [171] and spatially resolved electron energy loss spectroscopy $[108,172]$.

In this Letter, we demonstrate a straightforward method to image plasmonic modes with subwavelength resolution, and spectral and polarization discrimination. To this end, we arrange identical copies of plasmonic gold nanoparticles in a 2D array, and superpose this pattern with a geometrically incommensurable $2 \mathrm{D}$ array of dye doped polymer nanoparticles. The dye emission is spectrally tuned to the plasmonic resonance and thus serves as a sensitive probe of the plasmonic mode density [173]. As the distance between gold and polymer particles varies across the array, a microscale Moiré pattern is generated by the dye emission that corresponds to a magnified spatial map of the plasmonic mode.

Metal structures sustaining surface plasmons can substantially alter the excitation and emission rates of a nearby quantum system as, e.g., a fluorophore $[174,175]$. While excitation rates can be increased by the strong local plasmonic fields, the molecular emission rate is boosted in response to the high plasmonic LDOS according to Fermi's Golden Rule [4]. It has been shown recently that fluorophores with a large Stokes shift allow to access the emission channel modification only [173], thereby enabling direct measurements of the plasmonic LDOS. Here, we apply this approach to the high-resolution mapping of the plasmonic modes of lithographically tailored gold nanoparticles with resonance wavelengths in the red spectral range [black line in Fig. 10.1(a)].

Square gold nanoparticles (side length $100 \mathrm{~nm}$, height $50 \mathrm{~nm}$ ) were fabricated over an area of $100 \mu \mathrm{m} \times 100 \mu \mathrm{m}$, arranged in a regular square array with a grating constant 
a

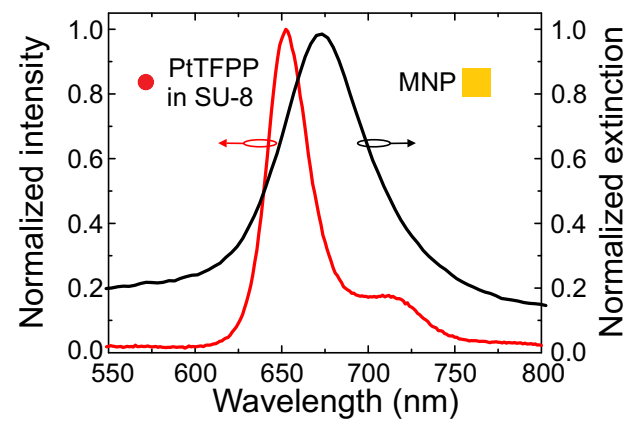

C

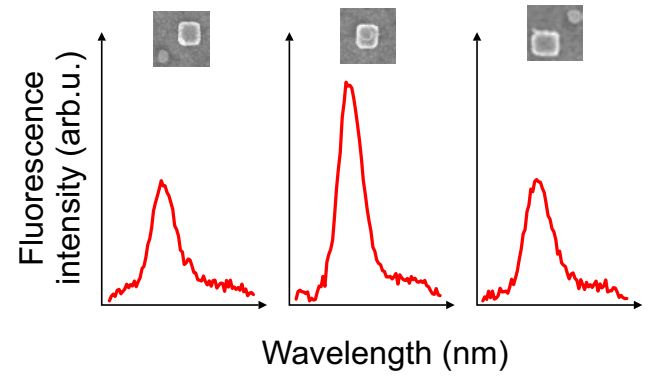

b
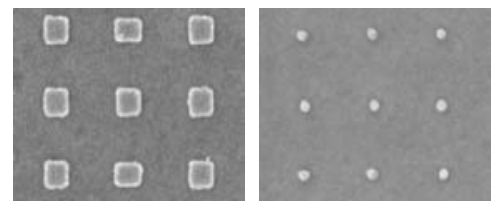

090

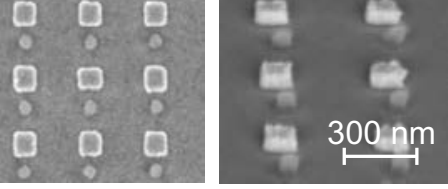

d

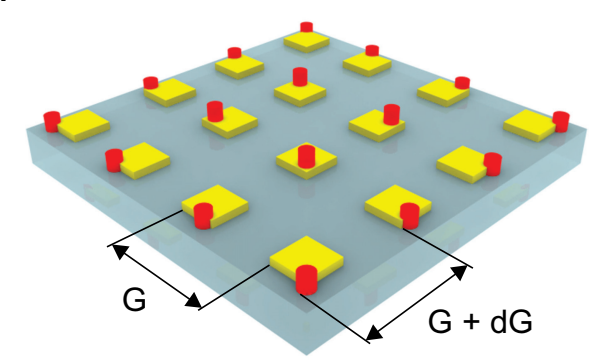

Figure 10.1.: (Color online) Principles of fluorescence Moiré mapping of gold nanoparticles. (a) Normalized plasmonic extinction (black curve) and photoluminescence spectra (red curve) of gold and SU8/PtTFPP nanoparticle arrays, respectively. (b) Scanning electron micrographs of (upper row) arrays of gold and SU8/PtTFPP nanoparticles and (lower row) a combined array of both nanoparticle types. (c) Photoluminescence spectra acquired from heterogeneous arrays of gold and SU8/PtTFPP nanoparticles. The relative mutual position of the nanoparticles within the array is shown in the insets for one particle pair. (d) Schematic sketch of distance variations of both types of nanoparticles within the combined arrays used for Moiré imaging, due to two different grating constants $\mathcal{G}$ and $\mathcal{G}+\mathrm{d} \mathcal{G}$.

of $\mathcal{G}=400 \mathrm{~nm}$ on indium-tin-oxide covered glass substrates by an electron beam lithography lift-off process relying on the positive resist poly(methyl methacrylate). We found the gold nanoparticles to be geometrically virtually identical throughout the array [upper left image in Fig. 10.1(b)]. Individual features in particle geometry deviating from the mean shape can amount to $\pm 3 \mathrm{~nm}$, limiting the achievable spatial resolution, while variations of the plasmonic resonance are negligible. As the fluorophore we chose a platinum porphyrin (PtTFPP) with an absorption band in the blue spectral range and an emission band centered at a wavelength of $650 \mathrm{~nm}$. In 
this case the plasmonic influence on the absorption rate is negligible [173]. PtTFPP was mixed at a concentration of $49.5 \mathrm{~g} / \mathrm{l}$ into the negative tone resist SU-8-3010 (MicroChem Corp.) which was diluted before with SU8-2000-thinner (MicroChem Corp.) at a ratio of 1:20. This concentration was found to yield homogeneously dispersed molecules with easily detectable fluorescence signal levels which are not strongly affected upon electron irradiation.

Electron beam exposure of a $60 \mathrm{~nm}$ thick PtTFPP doped SU8 film deposited by spin coating on indium-tin-oxide covered glass substrates yielded nanoparticles with a diameter of $40 \mathrm{~nm}$ and a height of $60 \mathrm{~nm}$, containing about 2000 PtTFPP molecules each. Again, the particles were arranged in a square array with $\mathcal{G}=400 \mathrm{~nm}$ over an area of $100 \mu \mathrm{m} \times 100 \mu \mathrm{m}$ [upper right image in Fig. 10.1(b)]. A photoluminescence spectrum of this array is shown by the red line in Fig. 10.1(a).

By combining both lithography schemes to an aligned two-step writing process, we fabricated heterogeneous arrays of gold and SU8/PtTFPP nanoparticles, as shown in the lower row of Fig. 10.1(b). SU8/PtTFPP particles positioned directly on gold particles showed about 2 times larger volumes as compared to particles sitting directly on the substrate which is most likely due to increased electron backscattering during the lithographic exposure process. We note that quenching in the case of SU8 particles in direct contact with a gold particle does not play a major role as only a few molecules are within quenching distance [174] due to the height of the SU8 particle.

The measured integral fluorescence intensity from the heterogeneous arrays depends on the mutual distance of gold and SU8/PtTFPP nanoparticles, thus giving a measure of the LDOS at the position of the molecules [Fig. 10.1(c)]. While the presence of the SU8 particle in principle causes a modification of the dielectric environment of the gold particle, measurements on arrays with different mutual distances of both types of particles show that no significant spectral modification of the plasmonic band takes place.

We next increased the grating constant for the SU8/PtTFPP array by $\mathrm{d} \mathcal{G}=2 \mathrm{~nm}$ to $402 \mathrm{~nm}$, while maintaining $\mathcal{G}=400 \mathrm{~nm}$ for the gold particle array. The distance between SU8/PtTFPP and gold nanoparticles forming neighboring pairs is thus shifted by $\mathrm{d} \mathcal{G}$ when moving from one pair to the next. The fluorescence emission intensity changes accordingly, spatially sampling the plasmonic LDOS [Fig. 10.1(d)]. Conse- 
quently, the fluorescence intensity distribution within the array as directly observable with a microscope is an image representing the LDOS of the (virtually identical) gold particles, with a magnification factor of $\mathcal{G} / \mathrm{d} \mathcal{G}=200$. The achievable spatial resolution is determined by the smallest possible step size in the lithographic process, and is not limited by diffraction.

The fluorescence measurements were performed with a conventional optical microscope equipped with a mercury lamp as the illumination source, band-pass filtered to $365 \pm 20 \mathrm{~nm}$. A $20 \times$, numerical aperture $=0.5$ microscope objective was used for both unpolarized sample illumination and fluorescence imaging onto a charge coupled device camera after passing a $515 \mathrm{~nm}$ high-pass filter.

An exemplary fluorescence image of a square gold nanoparticle under unpolarized illumination is shown in Fig. 10.2(a). The variation of the mutual distance between gold and SU8/PtTFPP nanoparticles is illustrated in the insets for five positions within the array. We observe a well defined square pattern in the fluorescence image, corresponding to the particle geometry. The ratio of the full-width-at-half-maximum of the fluorescence pattern (as defined in the cross-cut in Fig. 10.2(b) and the particle size is 210. While a comparison of the dimensions of the geometrical particle size and the plasmonic mode pattern is in principle meaningless, the found value is nevertheless close to the magnification value of 200 , as defined above, due to the strong localization of the plasmonic field to the particle surface. The curvature of the particle corners of about $5 \mathrm{~nm}$ is very well reproduced in the image, emphasizing nanoscale spatial resolution.

A key advantage of our method is the direct access to polarization-dependent and spectroscopic information by simply introducing a polarizer and spectral filters into the optical detection branch. Therefore additional filters were used for spectral selectivity (band pass $650 \pm 10 \mathrm{~nm}, 670 \pm 10 \mathrm{~nm}$ and long pass $\geq 720 \mathrm{~nm}$ ), and a polarizer in front of the camera allowed to analyze polarization dependencies. Fig. 10.3(a) shows a series of images of the square gold nanoparticle as in Fig. 10.2(a) but with both polarized and spectrally filtered detection, indicated to the left and on top of the image, respectively. Depending on polarization the mode pattern appears rotated by $90^{\circ}$, as expected for a dipolar excitation. The spectrally filtered images reveal subtle changes when moving the spectral window over the plasmonic resonance at 
a

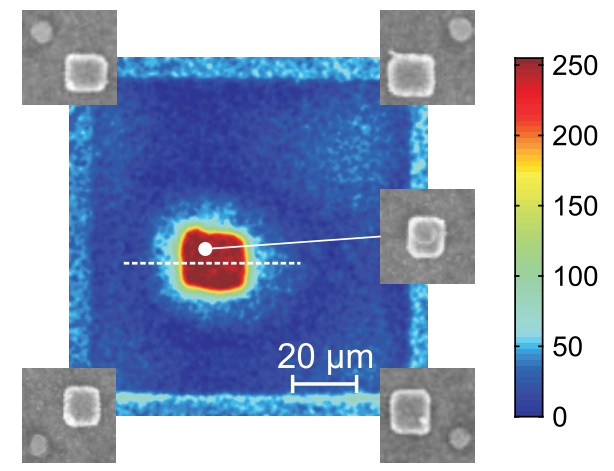

b

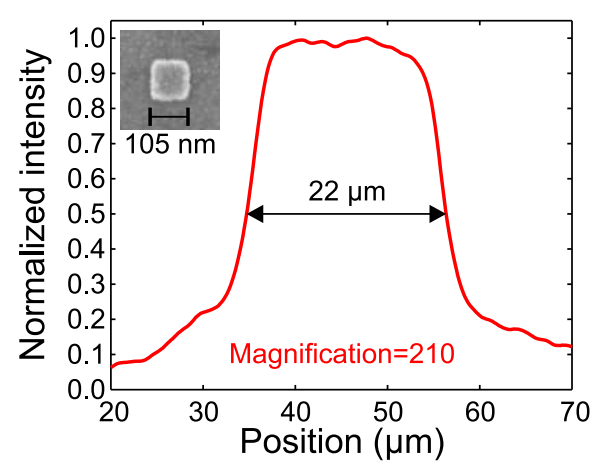

Figure 10.2.: (Color online) Fluorescence Moiré mapping of a gold nanoparticle. (a) Image of a square gold nanoparticle (side length $100 \mathrm{~nm}$, height $50 \mathrm{~nm}$ ) within an $100 \mu \mathrm{m} \times 100 \mu \mathrm{m}$ large array containing $250 \times 250$ particles. Unpolarized excitation at wavelengths of $365 \pm 20 \mathrm{~nm}$ and detection through a high-pass filter with a cut-off wavelength of $515 \mathrm{~nm}$ were applied. The scanning electron micrographs in the insets illustrate the variable distance between gold and SU8/PtTFPP nanoparticles, according to their position within the array. (b) Cross-cut along the dashed line in (a) revealing a ratio of Moiré image size and geometrical particle size (inset) of 210 .

$670 \mathrm{~nm}$ [Fig. 10.1(a)]. Both to the short-wavelength side of the resonance (band-pass filter $650 \pm 10 \mathrm{~nm}$ ) and on-resonance (band-pass filter $670 \pm 10 \mathrm{~nm}$ ) the plasmonic mode appears strongly localized to the particle while on the long-wavelength side (long-pass filter $\geq 720 \mathrm{~nm}$ ) the dipolar mode pattern extends further away from the particle. In addition, we observe bright and dark areas in between the nanoparticles corresponding to areas of enhanced and supressed LDOS, to be discussed further below. Areas of increased LDOS are particularly strong for the wavelength band $650 \pm 10 \mathrm{~nm}$, according to a radiating grating order at near-grazing angles [176]. We note that the intensity levels in the images are normalized to optimum contrast as molecular bleaching taking place during image acquisition (corresponding to about $5 \%$ intensity decrease) makes the comparison of absolute signal levels impossible.

For closer analysis of the experimental data we performed simulations based on the Boundary Element Method (BEM) [56, 82], which does not introduce any fitting parameters. The dielectric function of gold was extracted from optical data [41]. As the presence of a substrate was not taken into account in the calculations we assumed a homogeneous medium with a refractive index of 1.5. The geometry of the modeled 


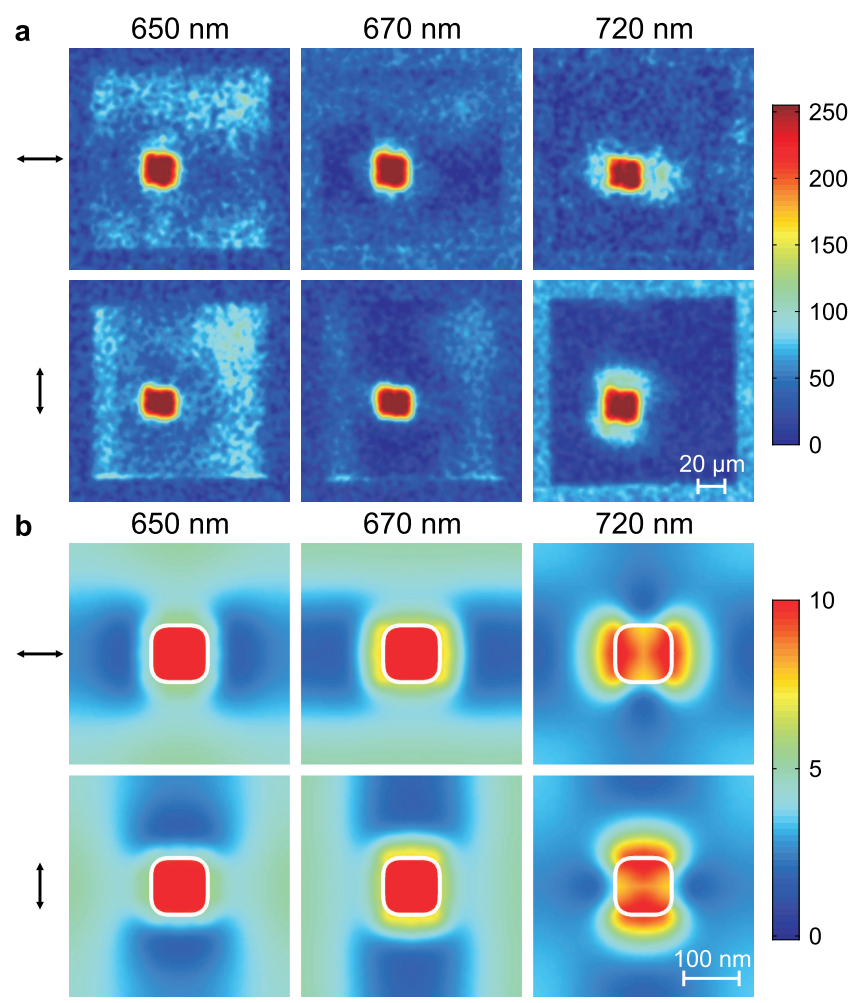

Figure 10.3.: (Color online) Polarization and wavelength dependence of the plasmon mode of a square gold nanoparticle. Polarization directions and wavelength ranges are given to the left and on top, respectively. (a) Experimental images for (upper row) horizontal and (lower row) vertical polarization and spectral ranges (left to right) $650 \pm 10 \mathrm{~nm}, 670 \pm 10 \mathrm{~nm}$ and $\geq 720 \mathrm{~nm}$. The color-encoded intensities in each image are normalized to optimum contrast and thus cannot be compared from one to another. (b) BEM simulations for the polarization directions indicated to the left and for discrete wavelengths of (from left to right) $650 \mathrm{~nm}, 670 \mathrm{~nm}$ and $720 \mathrm{~nm}$.

gold nanoparticles $\left(100 \times 100 \times 50 \mathrm{~nm}^{3}\right.$ for the square particles and $100 \times 300 \times 50$ $\mathrm{nm}^{3}$ for the nanorods) were chosen to match the measured extinction spectra. To account for the regular particle arrangement we simulate an ensemble of four gold nanoparticles arranged according to the experimental conditions, i.e., $\mathcal{G}=400 \mathrm{~nm}$. We model the molecules as dipole resonators, with moment $\boldsymbol{d}$, which are situated at positions $\boldsymbol{r}$. To account for the off-resonant excitation, we assume that the molecule is initially excited and subsequently decays, either through electromagnetic coupling 
or via intra-molecular channels. The electromagnetic decay rate $\gamma_{\mathrm{em}}(\boldsymbol{d}, \boldsymbol{r}, \omega)$ at a given photon energy $\hbar \omega$ is obtained from the imaginary part of the dyadic Green function [4], and the intra-molecular decay rate $\gamma_{\text {mol }}$ is related to the quantum yield of the isolated molecule, which we estimate to be about ten percent [177]. With $\gamma_{\text {rad }}^{\Omega, \ell}$ denoting the differential cross section for light emission into a sphere segment $\Omega$, which corresponds to the experimental conditions, and $\ell$ being the polarization, we can express the measured luminescence intensity, governed by the plasmonic LDOS, as

Moiré luminescence intensity

$$
I_{\text {lum }}^{\Omega, \ell}\left(\mathbf{R}_{\mathbf{0}}, \omega\right) \propto\left\langle\frac{\gamma_{\mathrm{rad}}^{\Omega, \ell}(\mathbf{d}, \mathbf{r}, \omega)}{\gamma_{\mathrm{em}}(\mathbf{d}, \mathbf{r}, \omega)+\gamma_{\mathrm{mol}}}\right\rangle .
$$

This quantity describes the probability that an excited molecule emits a photon into the direction of the photodetector. The brackets denote an ensemble average over the dipole orientations and positions $\boldsymbol{r}$ within the SU8 nanoparticle, centered at $\mathbf{R}_{\mathbf{0}}$. We assume a homogeneous distribution of randomly oriented dipole moments within a volume corresponding to the SU8 nanoparticle. The larger volume of the SU8/PtTFPP particles on top of the gold particles has been taken into account accordingly.

Fig. 10.3(b) shows maps calculated with Eq. (10.1) which directly correspond to the experimental images in Fig. 10.3(a). We emphasize that the simulation shows a single particle while in the experiment a magnified image including many nanoparticles is observed. From the comparison of the according images in Fig. 10.3(a) and (b) the agreement of experiment and simulation is evident, which we take as direct proof of the LDOS imaging properties of our system. In particular, the evolution of the spatial profile of the LDOS pattern as a function of observation wavelength is well reproduced. We found that the dark spots in between the particles occur at the positions where the electric field of the induced surface plasmon interferes destructively with the driving dipole of the molecule. Due to the phase difference 
between driving dipole and driven molecule, the composite system has a smaller effective dipole, thus corresponding to a smaller LDOS.

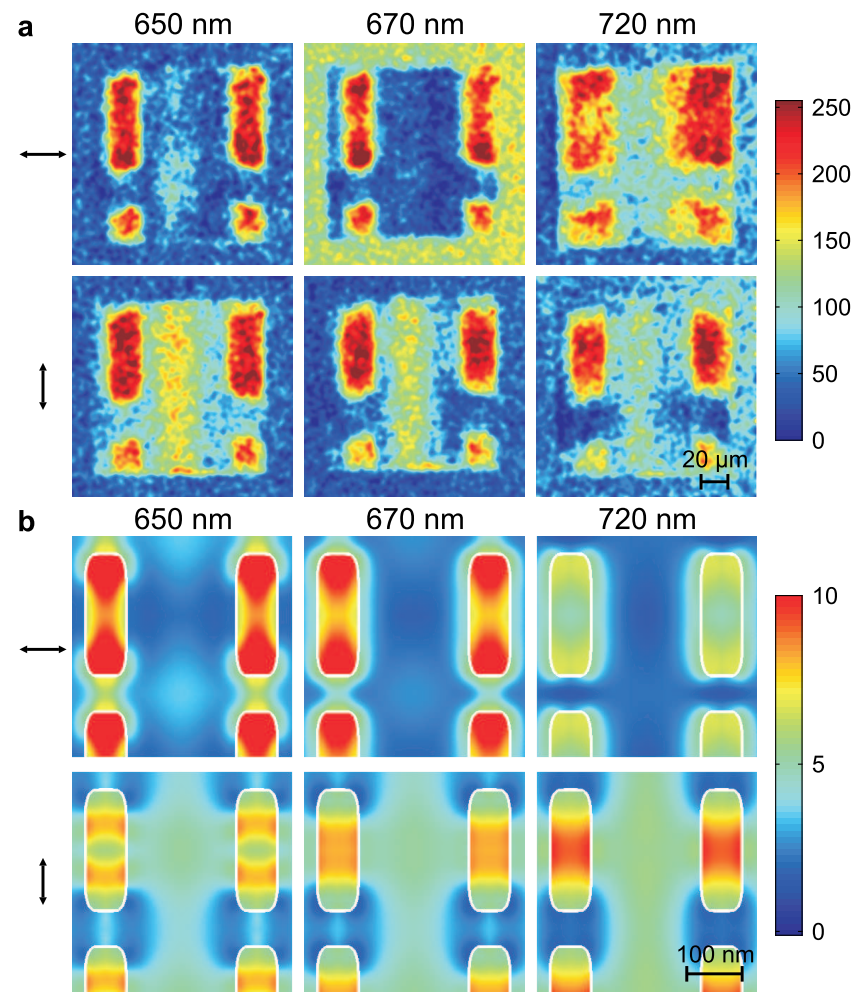

Figure 10.4.: Polarization and wavelength dependence of the plasmon mode of a gold nanorod. (a) Experimental images as in Fig. 10.3, but for a gold nanorod with $300 \mathrm{~nm}$ length, $100 \mathrm{~nm}$ width and $50 \mathrm{~nm}$ height. The intensities in each image are normalized to optimum contrast and thus cannot be compared from one to another. (b) BEM simulations for the polarization directions indicated to the left and for discrete wavelengths of (from left to right) $650 \mathrm{~nm}, 670 \mathrm{~nm}$ and $720 \mathrm{~nm}$.

Next, we investigate gold nanorods with a cross section diameter and length of 100 and $300 \mathrm{~nm}$, respectively, and a height of $50 \mathrm{~nm}$, arranged in a square pattern with $\mathcal{G}$ $=400 \mathrm{~nm}$. The dipolar resonances for excitation light polarizations along the short and long nanorod axes lie at a wavelength of $660 \mathrm{~nm}$ and in the infrared spectral range, respectively. The relative positions of nanorods and SU8/PtTFPP nanoparticles throughout the array were chosen such that two entire and part of two further nanorods appear in the images. Fig. 10.4(a) shows the experimental images, for experimental parameters as in Fig. 10.3. For polarization along the short nanorod axis 
we again find strongly localized mode patterns for the short-wavelength side of the resonance and on-resonance. The corresponding calculations in Fig. 10.4(b) reveal these patterns as due to excitations of a dipolar plasmonic mode along the short axis of the nanorod. For the off-resonant case (wavelengths $\geq 720 \mathrm{~nm}$ ) less confined fields are observed, again corresponding to the simulation. Choosing the polarization direction along the nanorod axis we find that the fluorescence signal becomes more confined to the center of the nanorod when increasing the observation wavelengths. The comparision with the simulations in Fig. 10.4(b) reveals the likely explanation for this effect. For wavelenghts $\geq 720 \mathrm{~nm}$ a non-resonant excitation of the dipolar resonance (in the infrared spectral range) dominates, giving rise to a rather localized mode pattern. For a wavelength of $650 \mathrm{~nm}$, however, the quadrupolar mode prevails, corresponding to a spatially more extented mode. Again, the fluorescence maxima in between the nanorods can be explained due to the grating effect.

The superresolution access to plasmonic modes by fluorescence Moiré mapping as discussed here can in principle be extented to any nanooptical system. While SU8/ PtTFPP nanoparticles have been found to be a particularly stable probe for the LDOS, probing other wavelength ranges on the basis of different fluorophores is straightforward. The challenge of well-controlled pattern fabrication could possibly be met by nanoimprinting based processes. Besides LDOS mapping and nanoparticle/quantum emitter studies, such systems could be useful for applications in sensors or wavelength and polarization encoded security features.

The GASS of NAWI Graz is acknowledged for financial support. 


\title{
11. PAPER: High-Resolution Surface Plasmon Imaging of Gold Nanoparticles by Energy Filtered Transmission Electron Microscopy
}

\author{
Bernhard Schaffer ${ }^{1,2,3}$, Ulrich Hohenester ${ }^{4}$, Andreas Trügler ${ }^{4}$, and Ferdinand Hofer ${ }^{1}$ \\ ${ }^{1}$ Institute for Electron Microscopy and Fine Structure Research, Graz University of Technology, 8010 Graz \\ 2 SuperSTEM Laboratory, STFC Daresbury, Keckwick Lane, WA4 4AD, Warrington, United Kingdom \\ ${ }^{3}$ Department of Physics and Astronomy, University of Glasgow, G12 8QQ, Glasgow, United Kingdom \\ ${ }^{4}$ Institut für Physik, Karl-Franzens-Universität Graz, Universitätsplatz 5, 8010 Graz, Austria
}

Published in: Phys. Rev. B 79, 041401(R) (2009)

\begin{abstract}
We demonstrate the imaging capabilities of energy-filtered transmission electron microscopy at high energy resolution in the low energy loss region, reporting the first direct image of a surface plasmon of an elongated gold nanoparticle at energies around $1 \mathrm{eV}$. Using complimentary model calculations performed within the boundary element method approach we can assign the observed results to the plasmon eigenmodes of the metallic nanoparticle.
\end{abstract}




\subsection{Introduction}

Plasmonics is an emerging field with numerous applications [5, 178], ranging from optical data processing $[14,15]$ over negative-refraction materials $[10,11]$ to biosensors $[8,9]$. Surface plasmons, the workhorse of plasmonics, allow to bridge between the micro- and nanometer length scales of conventional optics and nano-devices. This is achieved by binding or converting light to coherent electron charge oscillations, confined to the surface of metallic nanostructures. When optical emitters, such as molecules or quantum dots, are placed in the vicinity of metallic nanoparticles, they couple strongly to the evanescent fields of the surface plasmons, and the light-matter coupling becomes significantly enhanced. This is exploited in surface enhanced Raman scattering [179] or surface enhanced fluorescence [180], as well as in single-plasmon generation [16].

The electromagnetic enhancement is particularly strong at the so-called "hot spots", with typical sizes of a few nanometers, which are usually located in the gaps between two nanoparticles or at the sharp edges of single particles. While enhancement factors of several orders of magnitude are predicted [4], the analysis of the experimental data is often cumbersome and controversial due to the limited spatial resolution provided by optical far- and nearfield techniques, as well as due to the uncertainties regarding the precise location of the optical emitters. An alternative experimental technique, which allows to bypass these difficulties, is EELS in combination with scanning transmission electron microscopy (STEM) [108]. Here, a high-energy electron beam, with a transversal extension on the sub-nanometer scale, passes in the vicinity or through the nanoparticle, and some electrons lose energy through plasmon excitations, which are subsequently monitored. By raster scanning the beam over the metallic nanoparticle, one obtains information about the photonic local density of states of the metallic nanoparticles $[107,108,181]$. The spatial resolution of the maps is limited to the nanometer range by delocalization of the inelastic scattering [182].

The drawbacks of the EELS technique have so far been the energy cutoff in the lowenergy regime and the limited spatial resolution in large-area mappings. On the one hand, the so-called zero-loss peak (ZLP) of the electron beam usually masks the lowenergy part of the spectrum. This hinders the direct observation of surface plasmons in the red and infra-red regime, which is particularly appealing for (bio)sensors or 
applications at the telecom wavelengths. On the other hand, as STEM EELS data are sequentially acquired point-by-point, the sampling of the spatial dimensions is limited by keeping the total acquisition time low, which leads to usually rather coarse maps.

In this Letter we show that EFTEM as an alternative acquisition technique enables us to directly image surface plasmons at low energies. As a parallel imaging technique, which gathers images using scattered electrons of a specific energy loss range only, it achieves high spatial sampling easily and fast, while providing the same information content as STEM EELS $[183,184]$. The energy resolution of the EFTEM technique is typically in the range of a few $\mathrm{eV}$, but can be pushed to the sub-eV range if a small energy-filtering slit width and appropriate data correction schemes are used [185]. This enabled us to directly monitor surface plasmons at energies around or below $1 \mathrm{eV}$ without employing any deconvolution procedure. We achieved image sizes of $512 \times 512$ pixels for exposure times on the minute scale.

\subsection{Experiment}

In our experiments we used a Wien-filter monochromated FEI Tecnai F20 microscope with a High Resolution Gatan Imaging Filter and an UltraScan CCD camera [186]. The investigated specimen was a rod-shaped gold nanoparticle synthesized by a modification of the conventional citrate reduction of gold salt in water [187]. A holey carbon film was used as the sample support grid. Figure 11.1(a) displays the particle in a TEM bright field image. With the monochromated beam, the condenser lens was adjusted such that a homogeneous illumination of the particle could be achieved. An EFTEM image series was acquired via our customized acquisition script, which adapts exposure times and multiple frame read outs for each energy step to the encountered intensity situation [188]. We used an energy selecting slit width of $0.3 \mathrm{eV}$, and recorded the images in the energy range from 1.0 to $4.5 \mathrm{eV}$ in $0.1 \mathrm{eV}$ steps. The spatial sampling of the images was $512 \times 512$ pixels for a field-of-view of $456 \times 456 \mathrm{~nm}$, and the total acquisition time was 17 minutes. The raw data were then processed to correct for spatial drift, energy drift, and non-isochromaticity [185]. 

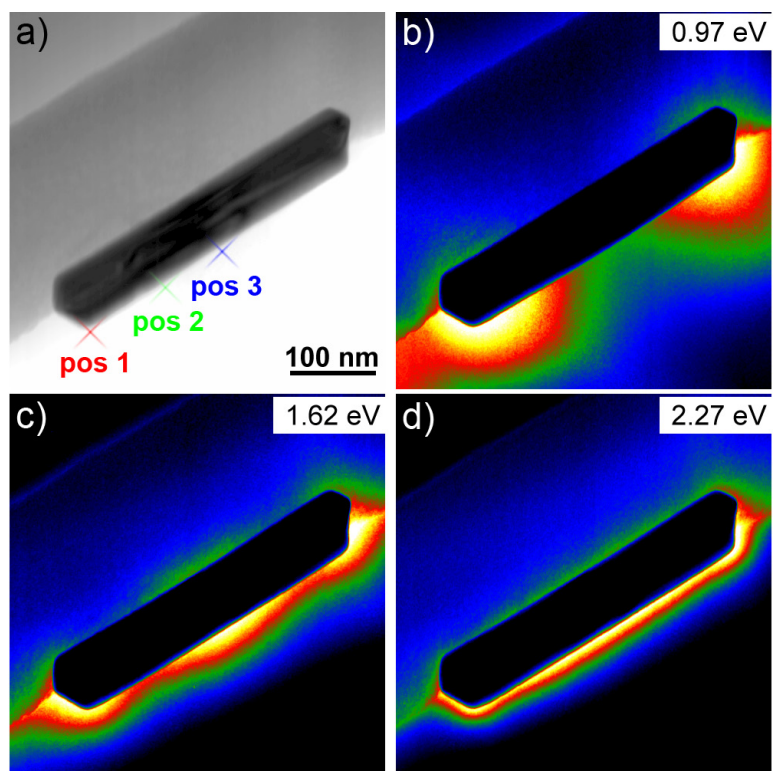

Figure 11.1.: (Color online) (a) TEM bright field image of rod-shaped gold nanoparticle, with approximately $400 \mathrm{~nm}$ length and $75 \mathrm{~nm}$ diameter. The markers indicate pixel positions at which EELS spectra in Fig. 11.2 have been extracted. (b-d) EFTEM images at given energy loss values. We use pseudocolor for better visibility. The EFTEM maps show the electromagnetic eigenmodes of the gold nanorod.

We first extracted, from single pixels of the EFTEM image series, energy loss spectra at the three positions indicated in Fig. 11.1(a). Figure 11.2(a) shows the corresponding spectra. For comparison, we also measured three EELS point spectra at approximately the same positions, which are shown in panel (b). In the spectra we observe three distinct peaks in the energy range below $3 \mathrm{eV}$, which we tentatively ascribe to the surface plasmon eigenmodes of the gold nanorod. This interpretation is supported by the EFTEM images of Fig. 11.1(b-d), taken at the energies of the peak maxima. The mode at $0.97 \mathrm{eV}$ has its intensity maxima at the top and bottom end of the rod, reminiscent of a dipolar excitation, while the map depicted in panel (c) shows an additional peak in the central region of the nanorod, similar to the first excited mode of a linear antenna. Finally, the mode at $2.27 \mathrm{eV}$, panel (d), vaguely shows two maxima at approximately one and two thirds of the long nanorod axis. In all cases we observe a strongly reduced intensity at the sides of the carbon support, in comparison to the vacuum side, which we attribute to absorption losses of the 
electron beam in the carbon.

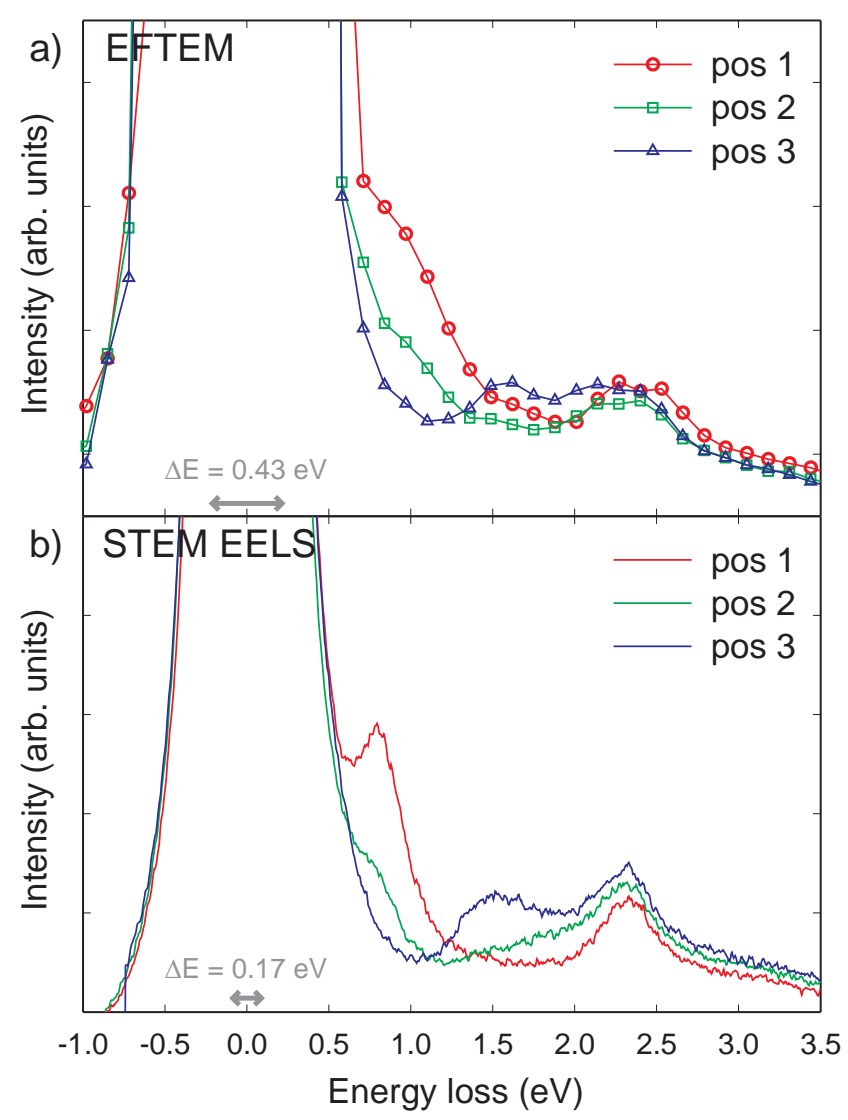

Figure 11.2.: (Color online) (a) Single-pixel extracted EELS spectra from EFTEM data at positions indicated in Fig. 11.1(a). (b) STEM EELS spectra acquired at the same sample positions. Gray bars indicate the energy resolution of the data.

\subsection{Theory}

To unambiguously interpret our experimental results, we additionally performed simulations using the Boundary Element Method (BEM) approach [82, 189]. In our simulations we assume that a beam of $200 \mathrm{keV}$ electrons passes in the vicinity of the metallic nanoparticle, as depicted in Fig. 11.3(a). For simplicity no penetrating trajectories were considered [108]. We model the gold nanoparticle as a rod with 
$400 \mathrm{~nm}$ length and $75 \mathrm{~nm}$ diameter, in accordance to the particle shape depicted in Fig. 11.1(a). The dielectric function of gold was extracted from optical data [41] and the refractive index of the surrounding medium was set to one. The external excitation due to the electron beam is described by the Liènard-Wiechert potentials [66]. We compute the dielectric response of the nanoparticle within the BEM framework by means of auxiliary surface charges and currents [82], which allow us to compute for a given frequency $\omega$ the induced electric field $\boldsymbol{E}_{\text {ind }}\left[\boldsymbol{r}_{e}(t), \omega\right]$ at the positions $\boldsymbol{r}_{e}(t)$ of the electron beam, and to express the energy loss probabilities according to [82]

$$
\Gamma(\omega)=\frac{1}{\pi \omega} \int \operatorname{Re}\left\{e^{-i \omega t} \boldsymbol{v} \cdot \boldsymbol{E}_{\text {ind }}\left[\boldsymbol{r}_{e}(t), \omega\right]\right\} d t .
$$

In our computational approach, we discretize the surface of the nanoparticle by a set of triangles, as shown in Fig. 11.3(a), and match the electromagnetic potentials at the triangle centers $[56,189]$. For spherical nanoparticles we compared the loss probability $\Gamma(\omega)$ with the analytic results of Mie theory [190], and found excellent agreement between the numerical and analytic results.
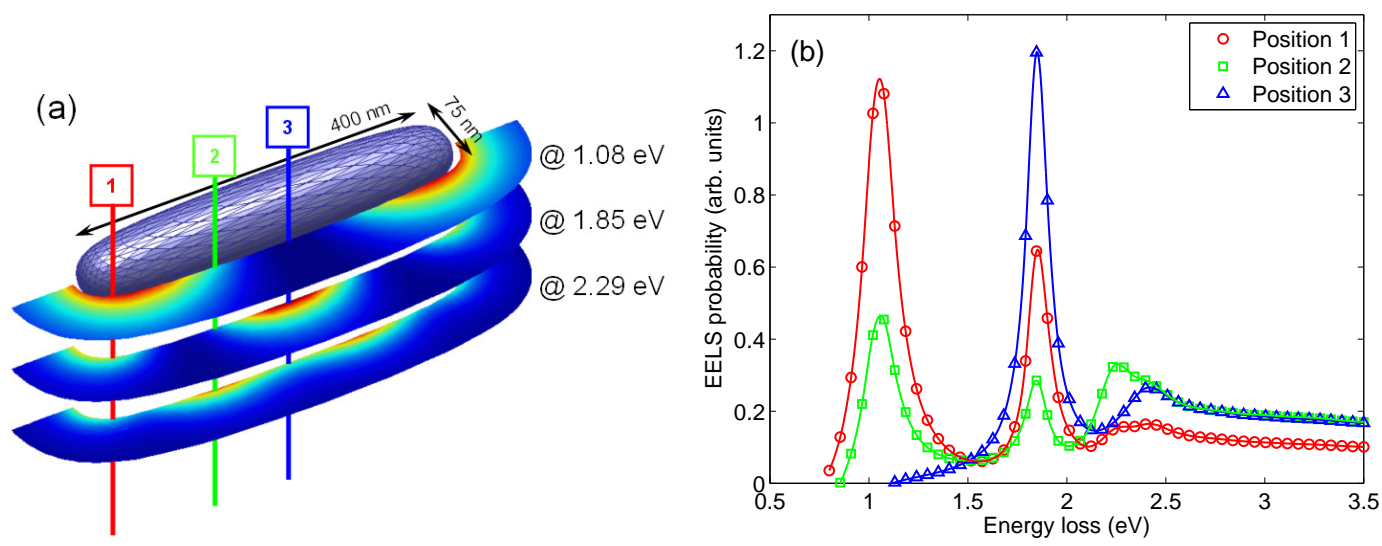

Figure 11.3.: (Color online) Results of BEM simulation. (a) Triangulated surface of rod-shaped nanoparticle and EFTEM maps as computed according to the prescription of Ref. [82]. The maps at the energies of $1.08 \mathrm{eV}, 1.85 \mathrm{eV}$, and $2.29 \mathrm{eV}$ have been displaced for better visibility. The lines indicate the direction of the electron beam. (b) Energy loss probability at the positions indicated in panel (a).

Results of our BEM simulation are shown in Fig. 11.3. Plasmon peak maxima are found at the energy positions of $1.08 \mathrm{eV}, 1.85 \mathrm{eV}$, and approximately $2.29 \mathrm{eV}$. The 
spatial intensity distribution of the plasmonic modes fits the experimental findings very well, giving evidence that the detected peaks in the EELS spectrum can be interpreted as the resonance modes of the plasmon oscillations of the gold nanoparticle. We attribute the differences between the energetic positions of the peak maxima in theory and experiment to our neglect of the holey carbon film in the simulations, which is expected to red-shift the peak maxima and to introduce an anisotropy in the simulated EFTEM maps. As regarding the peak at $2.29 \mathrm{eV}$, we find that it is strongly broadened. This is attributed to interband scatterings in gold, that take place above a threshold of approximately $2 \mathrm{eV}$. We also found that the EFTEM map of the $2.29 \mathrm{eV}$ peak is smeared out when we compute the loss probabilities not at a single energy, but integrate over an energy window of $0.4 \mathrm{eV}$ to simulate the effect of the limited energy resolution in the experiments.

\subsection{Discussion}

The energy resolutions of both EFTEM and EELS is governed by the width of the ZLP. Surface plasmons of silver nanoparticles have been mapped by EELS in an energy regime above $1.70 \mathrm{eV}$, using cold field emission guns together with a deconvolution procedure applied to the raw data [108], but surface plasmons of gold particles, which lie closer to the ZLP, can only be observed with a monochromated system [191]. A monochromated beam has a truly symmetric energy distribution and no pronounced ZLP tails towards the low-loss regime, giving profound advantages over cold field emission guns in this energy range [192]. An analysis of the full width at half maximum of the ZLP, extracted from the data in the vacuum area, reveals for our setup an EFTEM energy resolution of $0.43 \mathrm{eV}$, to be compared with the EELS resolution of $0.17 \mathrm{eV}$. This higher spectral resolution of EELS is also apparent from Fig. 11.2, although in both cases the different plasmonic eigenmodes, whose spectral widths are usually of the same order of magnitude, can be easily discriminated.

The advantage of EFTEM lies in its high spatial resolution over large field-of-views. It is important to realize that any attempt to obtain mapped EELS images with comparable spatial sampling is not simply more cumbersome or time-consuming, but is probably doomed to failure for the following reason. A difficulty for extreme low- 
loss mapping is posed by the strong intensity difference between the ZLP and the adjacent low-loss region, which challenges the dynamic range of the detector. The situation is even worse for a monochromated source, as the ZLP maximum becomes stronger with decreasing peak width. While it is possible to choose a setup which truncates the most intense parts of the ZLP by shifting the spectrum just across the edge of the detector, this technique relies on the stability of the system with respect to energy offsets over the time of acquisition. Considering the pixel dwell time of about $0.6 \mathrm{sec}$, which includes both the exposure time of $0.1 \mathrm{sec}$ and the data readout of the detector, a spatial map of $512 \times 512$ data points would have taken close to 36 hours, during which energy drift must not have exceeded $0.5 \mathrm{eV}$. Such long time stability can hardly be achieved in any modern system. In contrast to the scanning beam approach, within EFTEM the energy dimension is sampled sequentially and the dynamic range problem close to the ZLP can be overcome by especially adapted acquisition routines [188]. A second advantage of EFTEM is its ability to rapidly image large areas at lower magnification, without tediously adjusting the electron beam to coarse sampling conditions. For instance, imaging the spatial distribution of the $1 \mathrm{eV}$ peak alone would have required only 1 minute, thus demonstrating the unique capability of mapping large field-of-views with spatial resolution on the nanometer scale. Furthermore, in a scanned beam approach spatial drift during acquisition can lead to map distortions, which can not be corrected or even unambiguously identified from the data alone, whereas spatial consistence of EFTEM images is immediately evident.

In conclusion, we have shown that EFTEM imaging can be used to directly and efficiently visualize the electromagnetic eigenmodes of surface plasmons in metallic nanoparticles. The technique surpasses the spatial resolution of optical measurements by more than one order of magnitude and still allows for large field-of-views to be investigated within several minutes. Therefore, detailed studies of assembled particles or structured devices become available, bridging the gap between highly resolved local measurements, such as STEM EELS, and optical microscopy techniques, which average over larger areas and smear out inhomogeneities on a nanometer scale. The technique can also be applied for fast screening of many particles with slightly different resonance frequencies, or to localize "hot spots" of strongest resonance in a device, prior to detailed local analysis. It could also be advantageous for beam 
sensitive samples, where high dose rates of a focused beam may alter the structure locally during the measurement, whereas rapid parallel illumination of the sample allows for snap shots of an undamaged specimen. It should be emphasized that a beam monochromator is paramount for resolving plasmon energies around and below $1 \mathrm{eV}$, which constitutes an important energy range for Au nanoparticles. We believe that EFTEM will not supersede the scanning EELS approach as a high-spatial-resolution and high-energy-resolution technique in general, but will amend its capabilities with respect to the spatial domain.

We thank Harald Ditlbacher and Franz Aussenegg for most helpful discussions. This work has been supported in part by the FWF under project P18136-N13, and by the Zukunftsfonds Steiermark. 



\title{
12. PAPER: Influence of surface roughness on the optical properties of plasmonic nanoparticles
}

\author{
Andreas Trügler, Jean-Claude Tinguely, Joachim Krenn, Andreas Hohenau, and \\ Ulrich Hohenester \\ Institut für Physik, Karl-Franzens-Universität Graz, \\ Universitätsplatz 5, $8010 \mathrm{Graz}$, Austria
}

Published in: Phys. Rev. B 83, 081412(R) (2011)

\begin{abstract}
For plasmonic nanoparticles, we investigate the influence of surface roughness inherent to top-down fabrication on the optical properties, and find that it has a surprisingly small influence on the position and width of the plasmon peaks. Our experimental observation is supported by simulations based on the boundary element method approach. Using a perturbation approach, suitable for metallic nanoparticles with a moderate degree of surface roughness, we demonstrate that the reason for this lies in motional narrowing where the plasmon averages over the random height fluctuations. Surface roughness in large arrays of identical nanoparticles, such as encountered in the context of metamaterials, is thus expected to not constitute a major roadblock.
\end{abstract}




\subsection{Introduction}

Plasmonics bridges the gap between the micrometer length scale of light and the length scale of nanostructures [45]. This is achieved by binding light to coherent charge density oscillations of metallic nanostructures, so-called surface plasmons, which allow to focus electromagnetic radiation down to spots with spatial dimensions of the order of a few nanometers [193]. Coupling of quantum emitters, such as quantum dots or molecules, with plasmonic nanostructures can strongly modify their excitation and emission properties, observable in fluorescence [180] or surface enhanced Raman scattering, [194] and offers a unique means for tailoring light-matter interaction at the nanoscale. This has found widespread applications ranging from (bio)sensors [7] and solar cells [20] to optical and quantum communication technology [195]. Plasmonic nanoparticles are also at the heart of the emerging fields of metamaterials and optical cloaking [196].

Huge advances in fabrication techniques over the last years allow nowadays to fabricate plasmonic nanostructures with well understood and predictable properties. Nevertheless, practically all metallic nanoparticles suffer from size inhomogeneities and nanoscale surface roughness, $[197,198]$ which results in deviations of the plasmonic properties from those of idealized nanoparticles [199]. Particularly top-down approaches for nanoparticle fabrication often involve vacuum deposition of the metal structures, which leads to polycrystalline particles with an apparent surface roughness [198]. Despite the important role of surface roughness, there is still little understanding about the impact of such imperfection on the optical properties. Recent publications report on the control of nanoscale roughness and its strong effect on the nanoparticles far- and near-field optical properties $[197,198,200]$. However, varying surface roughness is often accompanied by varying crystallinity, and therefore the results do not allow for a clear distinction between the contributions of the surface and the bulk.

In this Letter we provide evidence from experiment, theory, and simulation that a moderate amount of surface roughness has no significant impact on the far-field optical properties of metallic nanoparticles. We interpret this as a kind of motional narrowing, where the surface plasmon averages over the random height fluctuations of the metal surface, which leads to destructive interference and an overall small net 
effect. Our findings might be beneficial for the design of metamaterials, which rely on large ensembles of practically identical particles, as well as devices based on the far-field properties of plasmonic nanoparticles.

\subsection{Experiment}

We measured the scattering spectra of individual polycrystalline (rough) gold nanoparticles to probe the influence of unavoidable surface roughness on the plasmonic signature of nanoparticles of nominally identical shape. The particles were fabricated by electron beam lithography on an ITO covered glass substrate. The substrate was coated with a polymer resist, which was then exposed and chemically developed. Vacuum deposition of gold and a liftoff process lead to polycrystalline particles (crystallite size $\sim 20 \mathrm{~nm}$ ) of designed shapes, [201] but with apparent surface roughness, as shown in Fig. 12.1(b). The particles were probed in a dark field microscope, collecting the scattered light by a $40 \times, 0.75$ numerical aperture objective and analyzing it with a spectrograph.

Fig. 12.1(a) shows exemplarily scattering spectra of four individual $50 \mathrm{~nm}$ wide, $100 \mathrm{~nm}$ long, and $45 \mathrm{~nm}$ high gold nanorods. Although the exact particle shapes differ due to nanoscale roughness [Fig. 12.1(b)], the dipolar plasmon resonance positions and the spectral widths of the four particles under consideration differ less then $20 \%$ of the full width at half maximum. Similar results were also observed for other nanoparticle shapes (not shown). From this we conclude that surface roughness has a surprisingly small influence on the optical properties of plasmonic nanoparticles, at least in the regime where the roughness does not lead to a noticeable change of the particle aspect ratio.

\subsection{Simulation}

We additionally performed simulations based on the Boundary Element Method (BEM), [23, 56, 82, 83] using a dielectric function representative for gold [41] and a refractive index of $n_{b}=1.65$ for the substrate. The particle shapes in the $(x, y)$ plane were extracted from the SEM images [Fig. 12.1(c)]. We use a particle height of 

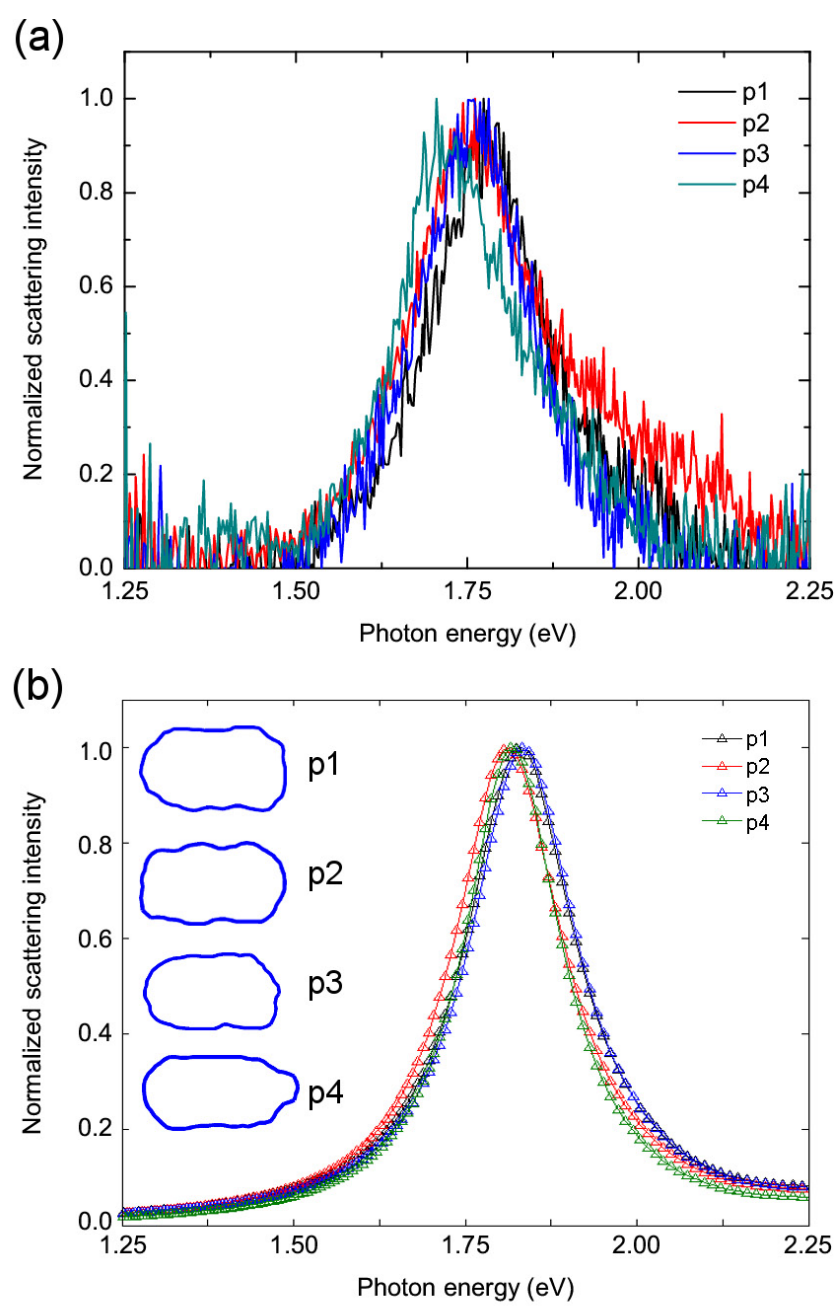

(c)

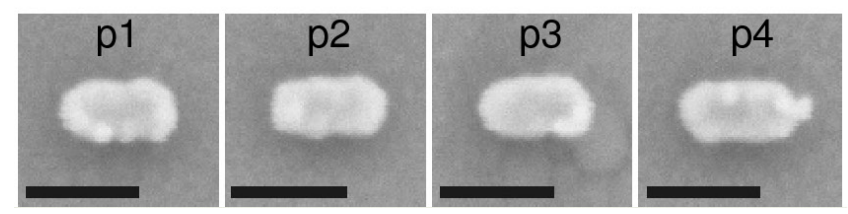

Figure 12.1.: (color online) (a) Scattering spectra of four individual gold nanorods excited with a polarization parallel to the particles long axis. (b) Spectra simulated within a BEM approach, using the gold dielectric function of Ref. [41] and a refractive index of $n_{b}=1.65$ for the substrate, for the particle shapes extraced from the SEM images reported in panel (c). The particle height is $45 \mathrm{~nm}$. The length of the scalebar in the lower panels is $100 \mathrm{~nm}$. 
$45 \mathrm{~nm}$ and round off the edges with a curvature radius of $5 \mathrm{~nm}$. Scattering spectra are reported in Fig. 12.1(b). In accordance to experiment, we find a surprisingly small influence of the particle shape on the plasmon peak positions. Also the absolute peaks heights (not shown) vary by less than 5 percent. We note that there are subtle differences between experiment and theory, such as the weak shoulder around $2 \mathrm{eV}$, which might be indicative of another excited mode sustained by its particular three-dimensional shape.

To inquire into the reasons for the observed behavior, in the following we use cylindrical nanorods as a showcase system, because of their extreme sensitivity to variations of the dielectric environment and shape [156]. We also employ the quasistatic approximation, $[82,85]$ which is justified for small particles and will allow us further below to introduce a perturbation analysis. With respect to the retarded BEM simulations, the quasistatic approximation is expected to lead to a slight overestimation of the plasmon peak energy.

In our simulations, we model surface roughness by adding stochastic height variations to the smooth surface of an ideal nanoparticle. In two dimensions and for a box with periodic boundary conditions, height variations with a Gaussian autocorrelation can be obtained by attaching to all Fourier coefficients arbitrary phase factors $e^{i \phi_{\text {rnd }}}$ viz.

$$
h(x, y)=\Delta h \Re e\left[\mathcal{F}^{-1}\left(e^{-\frac{1}{2} \varsigma_{h}^{2}\left(k_{x}^{2}+k_{y}^{2}\right)+i \phi_{\mathrm{rnd}}}\right)-\frac{1}{2}\right]
$$

where $\mathcal{F}^{-1}$ denotes the inverse Fourier transform and $\varsigma_{h}^{2}$ is the variance of the height fluctuations. We next map $h(x, y)$ to the nanoparticle surface and displace the vertices of the nanoparticle along the surface normal directions ${ }^{1}$. A typical realization of surface roughness is depicted in Fig. 12.2(b) for a nanorod.

Figure 12.2(a) shows spectra for gold nanorods for different height-to-diameter ratios. Throughout we set the rod diameter to $30 \mathrm{~nm}$ and $u s e \varsigma_{h}=3 \mathrm{~nm}$. For each spectrum we average over hundred random realizations of surface roughness and assume light

\footnotetext{
${ }^{1}$ Mapping can be faciliated by computing $h(x, y, z)$ in three dimensions, with an expression similar to Eq. (12.1), and interpolating the stochastic height variations $h$ to the nanoparticle surface.
} 


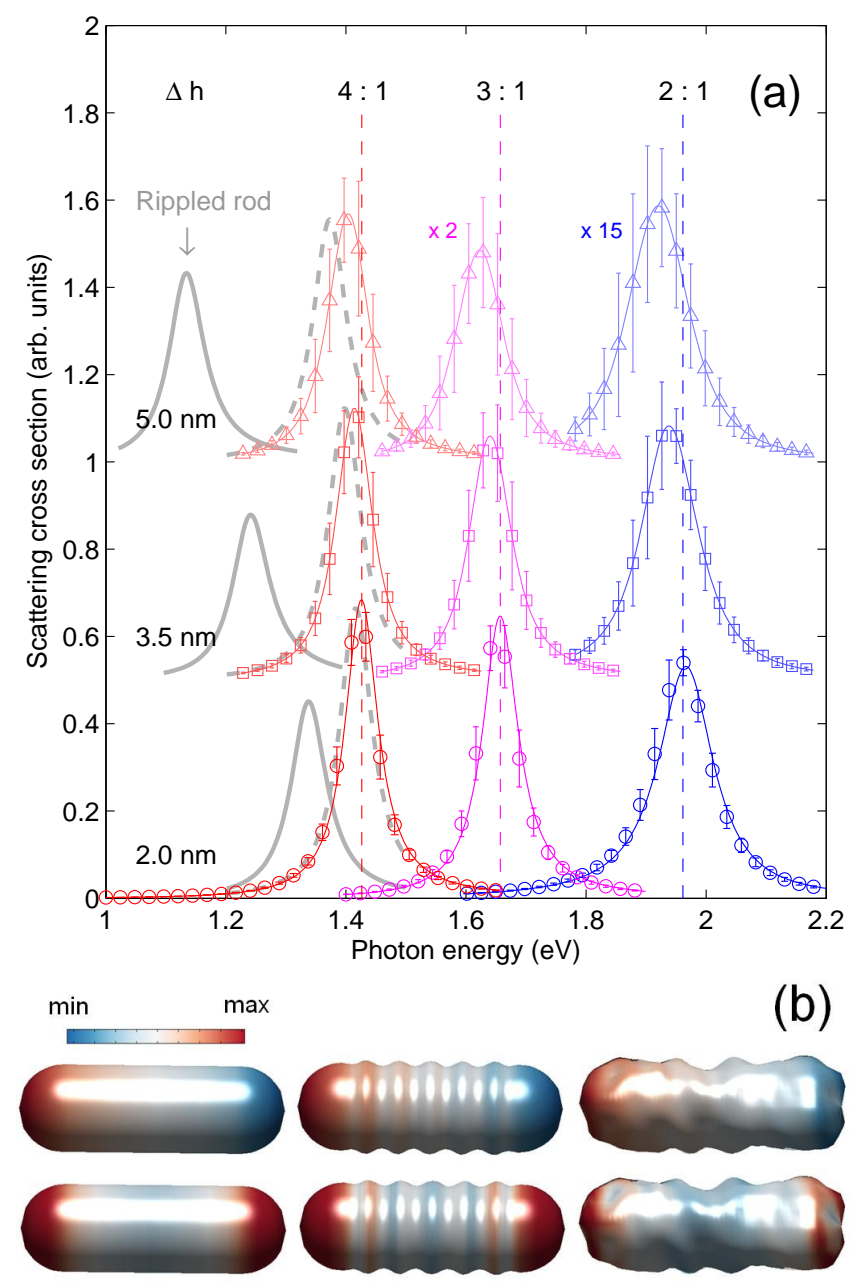

Figure 12.2.: (color online) (a) Simulated spectra for nanorods with a diameter of $30 \mathrm{~nm}$ and for height-to-diameter ratios of $2: 1,3: 1$, and $4: 1$. In our simulations we use a dielectric function representative for gold [41] and a homogeneous dielectric background $\left(n_{b}=1.5\right)$, and add stochastically surface roughness according to the prescription of Eq. (12.1) with $\varsigma_{h}=3 \mathrm{~nm}$ and for different $\Delta h$ values, which determine the height variations of surface roughness. Each spectrum is averaged over 100 randomly generated nanoparticles. The gray lines report results for rippled rods, as discussed in the text. The spectra for ratios $2: 1$ and $3: 1$ have been magnified by factors of 15 and 2, respectively. (b) Surface charge distributions $\sigma_{a}$ for the optically active plasmon modes for the smooth (left), rippled (middle), and rough (right) nanorods. 
excitation with a polarization along long axis of the rod. One clearly observes that when increasing the amount of surface roughness, by choosing different values of $\Delta h$, the shape of the averaged spectra does not change (with exception of an overall small red shift). Only the variance of the individual spectra increases with increasing $\Delta h$, as indicated by the errorbars. Thus, our numerical simulations again demonstrate that surface roughness has a surprisingly small influence on the optical properties of plasmonic nanoparticles.

Things change considerably if we use the more regular height variations of a rippled rod, depicted in Fig. 12.2, that was recently suggested as a viable model for surface roughness [202]. Here the spectra strongly shift to the red when $\Delta h$ is increased, as depicted by the gray lines on the left of Fig. 12.2. Thus, it appears that the irregular shape of the surface height fluctuations is responsible for the overall small shift of the plasmon peak position. Indeed, when ripples are introduced not only along the symmetry axis of the nanorod but also around its circumference (here 6 ripples) the redshift becomes strongly reduced, as shown by the dashed gray lines.

\subsection{Theory}

We next develop a perturbation analyis which will help us to understand the effects of surface roughness more deeply. Let us first recall the basic elements of the BEM approach. For a metallic nanoparticle with dielectric function $\varepsilon_{m}(\omega)$ embedded in a dielectric background with constant $\varepsilon_{b}$, the solutions of the Poisson equation within the two media are given by the Green function $G\left(\boldsymbol{r}, \boldsymbol{r}^{\prime}\right)=1 /\left|\boldsymbol{r}-\boldsymbol{r}^{\prime}\right|$. The electrostatic potential can be written in the ad-hoc form $\Phi=\langle G, \sigma\rangle+\phi_{\text {ext }}$ [82], where $\sigma$ is a surface charge distribution located at the boundary $\partial \Omega$ of the metallic nanoparticle, which has to be chosen such that the boundary conditions of Maxwell's equations are fulfilled, $\langle G, \sigma\rangle=\int_{\partial \Omega} G(\boldsymbol{r}, s) \sigma(s) d s$ defines an inner product, and $\phi_{\text {ext }}$ is the potential of the external perturbation.

Continuity of the normal component of the dielectric displacement at the metaldielectric interface gives an expression 


$$
\Lambda(\omega) \sigma+\left\langle\frac{\partial G}{\partial \hat{n}}, \sigma\right\rangle=-\frac{\partial \phi_{\mathrm{ext}}}{\partial \hat{n}}, \quad \Lambda=2 \pi \frac{\epsilon_{m}+\epsilon_{b}}{\epsilon_{m}-\epsilon_{b}}
$$

that can be used for the calculation of the surface charge distribution. Here $\partial G / \partial \hat{n} \equiv$ $F$ and $\partial \phi_{\text {ext }} / \partial \hat{n} \equiv \phi_{\text {ext }}^{\prime}$ denote the surface derivatives of the Green function and the external potential, respectively.

We next define the right and left eigenvectors $\sigma_{k}^{R}$ and $\sigma_{k}^{L}$ of the surface derivative of the Green function through $[85,86]$

$$
\left\langle F, \sigma_{k}^{R}\right\rangle=\lambda_{k} \sigma_{k}^{R}, \quad\left\langle\sigma_{k}^{L}, F\right\rangle=\lambda_{k} \sigma_{k}^{L},
$$

which form a biorthogonal set with $\left\langle\sigma_{k}^{L}, \sigma_{k^{\prime}}^{R}\right\rangle=\delta_{k k^{\prime}}$. The functions $\sigma_{k}^{R}$ can be interpreted as the surface plasmon eigenmodes, and the response to any external perturbation can be decomposed into these modes viz.

$$
\sigma=-\sum_{k} \frac{\sigma_{k}^{R}}{\Lambda(\omega)+\lambda_{k}}\left\langle\sigma_{k}^{L}, \phi_{\text {ext }}^{\prime}\right\rangle .
$$

Apparently, a given mode $k$ gives a noticeable contribution only if the coupling $\left\langle\sigma_{k}^{L}, \phi_{\text {ext }}^{\prime}\right\rangle$ to the external potential (in our case plane wave illumination) is sufficiently strong and if the denominator becomes small. The plasmon resonance condition translates to $\operatorname{Re}\left[\boldsymbol{\Lambda}(\omega)+\lambda_{k}\right]=0$. This is an extremely useful expression as it allows to separate the structural properties (described by $\lambda_{k}$ ) from the material properties (described by $\boldsymbol{\Lambda}$ ).

We are now in the position to analyze the effects of a moderate surface roughness, which we model as a distortion of the surface $\partial \Omega$ from its ideal shape. In turn, the surface derivative of the Green function $F$ changes to $F+\delta F$, where $\delta F$ is expected to have the same random character as the surface fluctuations ${ }^{2}$. How does $\lambda_{k}$, which determines the peak positions of the plasmons, change in case of surface roughness?

${ }^{2}$ This is because $F\left(s, s^{\prime}\right)$ is governed for $s \approx s^{\prime}$, where deviations of the ideal shape are most significant, directly by the surface curvature. 
When $\delta F$ is sufficiently small we can employ perturbation theory, in complete analogy to quantum mechanics. We treat $F$ as the unperturbed part and $\delta F$ as the "perturbation". Following Ref. [87] we introduce for a given plasmon mode $a$ the projector

$$
\frac{Q_{0}}{a} \equiv \sum_{k \neq a} \frac{\sigma_{k}^{R, 0} \sigma_{k}^{L, 0}}{\lambda_{a}^{0}-\lambda_{k}^{0}},
$$

where the superscript 0 indicates the eigenvalues and eigenfunctions for the ideal nanoparticle surface. The corrections to $\lambda_{a}$ then become within lowest order perturbation theory

$$
\begin{aligned}
\lambda_{a}^{1} & =\left\langle\sigma_{a}^{L, 0}, \delta F \sigma_{a}^{R, 0}\right\rangle \\
\lambda_{a}^{2} & =\left\langle\sigma_{a}^{L, 0}, \delta F \frac{Q_{0}}{a} \delta F \sigma_{a}^{R, 0}\right\rangle .
\end{aligned}
$$

From this we can draw an important conclusion for the dipolar modes, whose $\sigma_{a}^{R, 0}$ distribution usually varies smoothly on the length scale of surface roughness. Since Eq. (12.6a) averages the random variations $\delta F$ over the unperturbed distributions $\sigma_{a}^{R, 0}$, the positive and negative $\delta F$ values associated with the height variations of surface roughness will become effectively averaged out. This results in a small $\lambda_{a}^{1}$ correction, and consequently surface roughness does not affect significantly the optically active plasmon modes.

In a sense, this finding is similar to motional narrowing in semiconductor quantum wells [113], where the motion of an optically excited electron-hole pair (exciton) is subject to the potential induced by the local monolayer fluctuation inherent to quantum wells. When the exciton propagates through the well it "averages" over the fluctuations of the random potential, which results in a narrowing of the exciton lineshape.

In Fig. 12.3 we compare the results of our full simulations with the predictions of Eq. (12.6). The open and full symbols show the true resonance energies (obtained from $\operatorname{Re}\left[\boldsymbol{\Lambda}(\omega)+\lambda_{k}\right]=0$ ) and the perturbation results (obtained with $\lambda_{a}^{0}+\lambda_{a}^{1}$ and $\lambda_{a}^{0}+\lambda_{a}^{1}+\lambda_{a}^{2}$, respectively). Symbols on the diagonal correspond to the situation where perturbation theory and the full BEM simulations coincide. In all cases the 


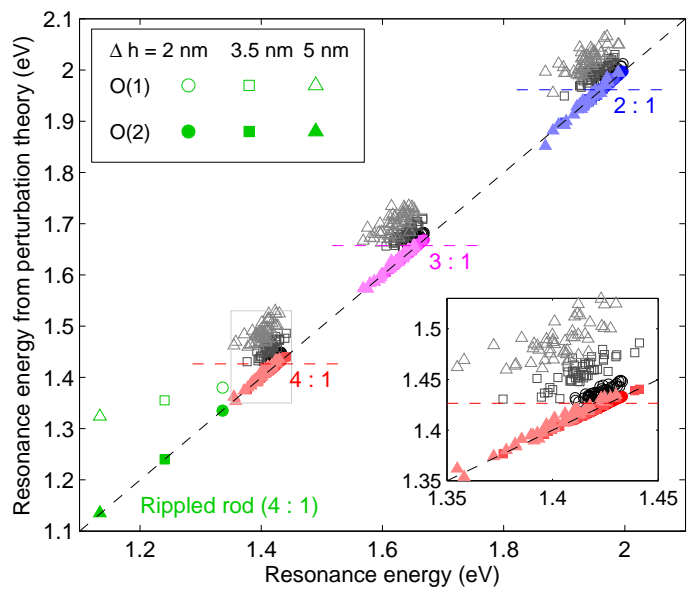

Figure 12.3.: (color online) Comparison of full simulations and perturbation theory of Eq. (12.6), for the positions of the resonance energies of the optically active surface plasmon modes with polarization along the long axis of the rod. The open and filled symbols report results obtained within first and second order perturbation theory, respectively. In the inset we show a blow-up for the nanorod with a height-to-diameter ratio of $4: 1$.

shift of the resonance with respect to the positions of the smooth nanorod (dashed horizontal lines) is small. Quite generally, first order perturbation theory, which ignores any variations of the surface charge distribution $\left(\sigma_{a}=\sigma_{a}^{0}\right)$, leads to a blue shift. Only for second order perturbation theory, which includes modification of $\sigma_{a}$, the red shift of the plasmonic resonances is properly reproduced.

\subsection{Summary}

In summary, we have investigated the influence of surface roughness on the optical properties of plasmonic nanoparticles, and have found a surprisingly small effect. Using a simulation and perturbation theory approach, we have been able to trace back our findings to a motional narrowing, where the plasmon averages over the random height fluctuations. As no corresponding conclusions prevail for the nearfield optical properties, our results do not invalidate the findings of "hot spots" in fluorescence or surface enhanced Raman scattering experiments. This work has been supported in part by the FWF under project No. P21235-N20. 


\title{
13. PAPER: Tailoring spatiotemporal light confinement in single plasmonic nanoantennas
}

\author{
Tobias Hanke ${ }^{1}$, Julijan Cesar ${ }^{1}$, Vanessa Knittel $^{1}$, Andreas Trügler ${ }^{2}$, \\ Ulrich Hohenester $^{2}$, Alfred Leitenstorfer ${ }^{1}$, and Rudolf Bratschitsch ${ }^{1}$ \\ 1 Dep. of Physics and Center for Applied Photonics, Univ. of Konstanz, Univ.str. 10, D-78457 Konstanz \\ 2 Institut für Physik, Karl-Franzens-Universität Graz,Universitätsplatz 5, 8010 Graz, Austria
}

(submitted)

\begin{abstract}
Plasmonic nanoantennas are efficient devices to concentrate light in spatial regions much smaller than the wavelength. Only recently, their ability to manipulate photons also on a femtosecond timescale has been harnessed. Nevertheless, designing the dynamical properties of optical antennas has been difficult since the relevant microscopic processes governing their ultrafast response have remained unclear. Here, we exploit frequency resolved optical gating to directly investigate plasmon response times of different antenna geometries resonant in the near infrared. Third-harmonic imaging is used in parallel to spatially monitor the plasmonic mode patterns. We find that the few-femtosecond dynamics of these nanodevices is dominated by radiative damping. A high efficiency for nonlinear frequency conversion is directly linked to long plasmon dephasing times. This single parameter explains the counterintuitive result that rod-type nanoantennas with minimum active volume generate by far the strongest third-harmonic emission as compared to the more bulky geometries of bowtie-, elliptical- or disc-shaped specimens.
\end{abstract}




\subsection{Light confinement in nanoantennas}

Metal nanoantennas hold great promise for applications in physics, chemistry and biology since they convert propagating light to highly localized and strongly enhanced electric fields $[178,203,204]$. Besides their favorable linear optical properties, nanoantennas have recently been shown to represent excellent nonlinear emitters of light [78,205-207]. In order to understand the mechanisms that govern nonlinear light emission on ultrafast timescales, steady-state characterization techniques such as twophoton photoluminescence [61,171,208-210], cathodoluminescence [211], dark field scattering spectroscopy [135,212], scanning near field optical microscopy [213-215] and electron energy loss spectroscopy [108,172] are insufficient. An extraordinary temporal resolution is required in addition to a high spatial selectivity since plasmonic effects in metal nanoantennas occur on a few-femtosecond timescale. Only recently, few-femtosecond fiber lasers became available generating pulse trains with unprecedented stability [216] and reaching the single-cycle limit [217]. Ultraprecision studies with parallel resolution both at the nanometer and femtosecond scales are enabled with these systems.

Here, we use third-harmonic spectroscopy to compare commonly used nanoantenna shapes with respect to their nonlinear emission of light. Surprisingly, the highest intensity is not linked to a specific shape, but to the smallest antenna volume. Nonlinear imaging with sub-wavelength resolution reveals that the fundamental dipole mode of the plasmon is excited in all antenna types. We find that resonant nanoantennas in the near-infrared are radiation-damped systems. The few-femtosecond plasmon damping time is the only parameter that governs the intensity of coherent nonlinear light emission of these single-mode nanodevices.

Tailor-made arrays of gold nanoantennas are fabricated by electron beam lithography on fused silica substrates of $200 \mu \mathrm{m}$ thickness. They consist of two arms separated by a feedgap. Their dimensions have been carefully chosen to be exactly resonant to femtosecond excitation with linear polarization parallel to the long axis. (Supplementary Information, Tab. 13.1 and Fig. 13.6). Few-cycle laser pulses in the near infrared are generated by a mode-locked Erbium-doped fiber laser system working at a repetition rate of $40 \mathrm{MHz}$. The average power is typically adjusted to be $5 \mathrm{~mW}$. Ultimate temporal resolution is achieved by an excitation bandwidth of $470 \mathrm{meV}$ ( 450 
$\mathrm{nm})$ and using a Cassegrain reflector $(\mathrm{NA}=0.65)$ for focusing. The nonlinear emission from single nanoantennas is collected in transmission geometry with a second objective $(\mathrm{NA}=0.95)$, short-pass filtered (Schott BG39), dispersed with a grating monochromator (Acton Spectra Pro 2300i) and detected with a Peltier-cooled CCD camera (Andor Newton DU 920P-BU).
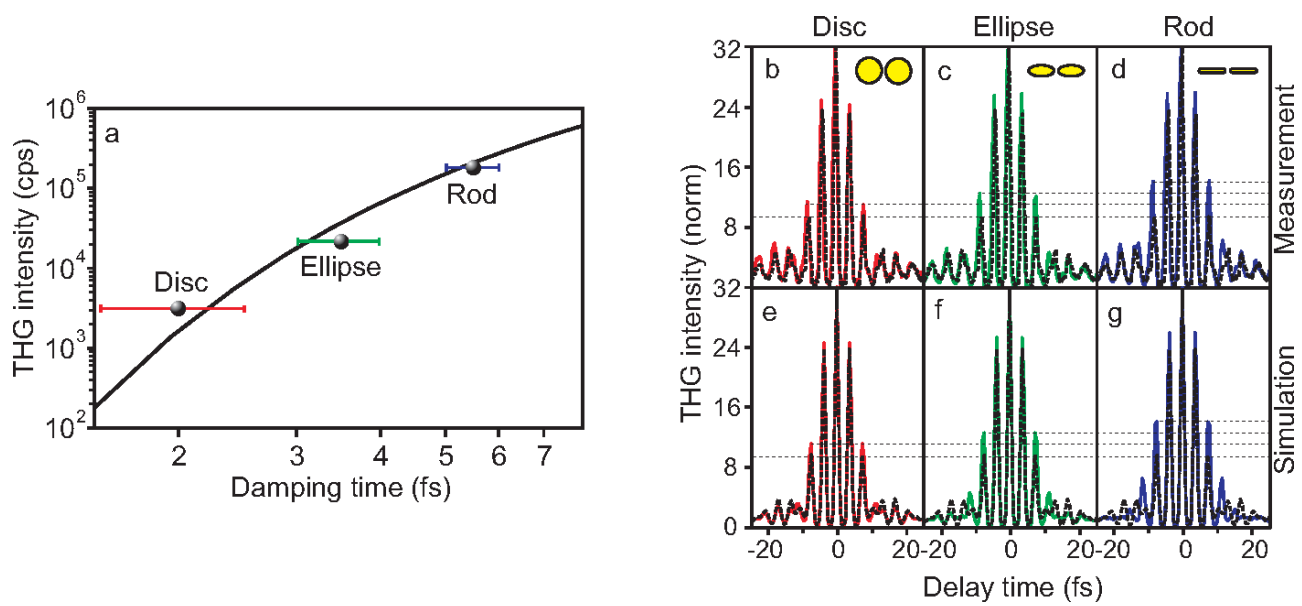

Figure 13.1.: Nonlinear emission and plasmon damping from metal nanoantennas of different shapes. a, Measured (black dots) and calculated (black line) thirdharmonic intensity depending on the plasmon damping time on a logarithmic scale. $\mathbf{b}-\mathbf{d}$, Measured and $\mathbf{e}-\mathbf{g}$, simulated interferometric autocorrelation traces of three resonant nanoantennas, each with the autocorrelation trace of the instantaneous response from the substrate (black dashed line) shown for comparison. The rod antenna $(\mathrm{d}, \mathrm{g})$ exhibits a plasmon damping time of $\tau=5.5 \mathrm{fs}$, the elliptical one (c, f) shows $\tau=3.5 \mathrm{fs}$, and $\tau=2.0 \mathrm{fs}$ for the disc-shaped antenna (b, e). Antenna dimensions are listed in Supporting Information, Tab. 13.1.

The most striking finding is the large intensity difference of the generated thirdharmonic emission for different antenna shapes (Fig. 13.1 a). Even though all antennas are resonant for excitation with light polarized along the long axis, the rod antenna generates one order of magnitude more third-harmonic intensity than the elliptical structure. Compared to the disc-shaped antenna, an increase of even two orders of magnitude is achieved. As we will show, the dynamical properties of the nanoantennas on the femtosecond timescale are key for understanding this phenomenon.

We use frequency resolved optical gating at the third-harmonic (THG-FROG) to measure the plasmon damping time $\tau$ of single nanoantennas directly in the time 
domain [78]. For this purpose, we collinearly excite the structures with two identical transients derived from a Mach-Zehnder interferometer and record the emitted THG signal as a function of time delay between the pulses. Fig. $13.1 \mathrm{~b}-\mathrm{d}$ show measured THG autocorrelation traces for three different antenna shapes. The electric field amplitude and phase of the driving laser pulse are retrieved from the THG-FROG measurement of the instantaneous response from the substrate surface. We simulate the autocorrelation traces (Fig. $13.1 \mathrm{e}-\mathrm{g}$ ) by assuming harmonic oscillation of the resonant plasmon with a single exponential decay [218]. The damping time constant $\tau$ represents the only free fit parameter. It is determined by comparison of the fringe amplitudes of the measured and simulated autocorrelation data. As may be seen in Fig. 13.1, the disc-shaped specimen exhibits the shortest plasmon dissipation time of $\tau=2$ fs. Note that this interval corresponds to only half a cycle of light at the fundamental resonance frequency of $250 \mathrm{THz}$. The elliptical design follows with $\tau=3.5$ fs. At $\tau=5.5 \mathrm{fs}$, the rod antenna shows the longest storage time for the radiation energy. Weak plasmon damping is associated with an effective build-up of the plasmon oscillation, if driven on resonance. Therefore, a strong third-harmonic emission results despite the small active volume of these devices. Indeed, the measured decay times may be used to calculate the relative third-harmonic intensity within the harmonic oscillator model. The excellent agreement between theory and experiment in Fig. 13.1 a clearly demonstrates that knowledge of the plasmon damping time alone suffices to predict the nonlinear emission intensity from different nanoantennas.

We now turn to the question what determines the plasmon damping time and how it can be engineered. One important issue in this context is whether the shape of the nanoantenna plays a significant role or not. It is conceivable that the excitation of differing or even multiple antenna resonances governs the observed changes in the plasmon damping time. Therefore, we analyze the plasmonic mode patterns of different nanoantenna types. Fig. $13.2 \mathrm{a}$ - e show scanning electron micrographs (SEM) of rod-, elliptical-, disc-, bowtie-, and cross-shaped antennas. Ultimate spatial resolution is achieved for nonlinear emission mapping by replacing the Cassegrainian $(\mathrm{NA}=0.65)$ by an oil immersion objective $(\mathrm{NA}=1.3)$. The ultrabroadband spectrum of the pump laser is restricted to a bandwidth of $0.17 \mathrm{eV}(225 \mathrm{~nm})$ centered at $0.97 \mathrm{eV}(1.28 \mu \mathrm{m})$. We still achieve a pulse duration as short as $24 \mathrm{fs}$ in the focus of the oil immersion objective (Supplementary Information, Fig. 13.7). The sample 

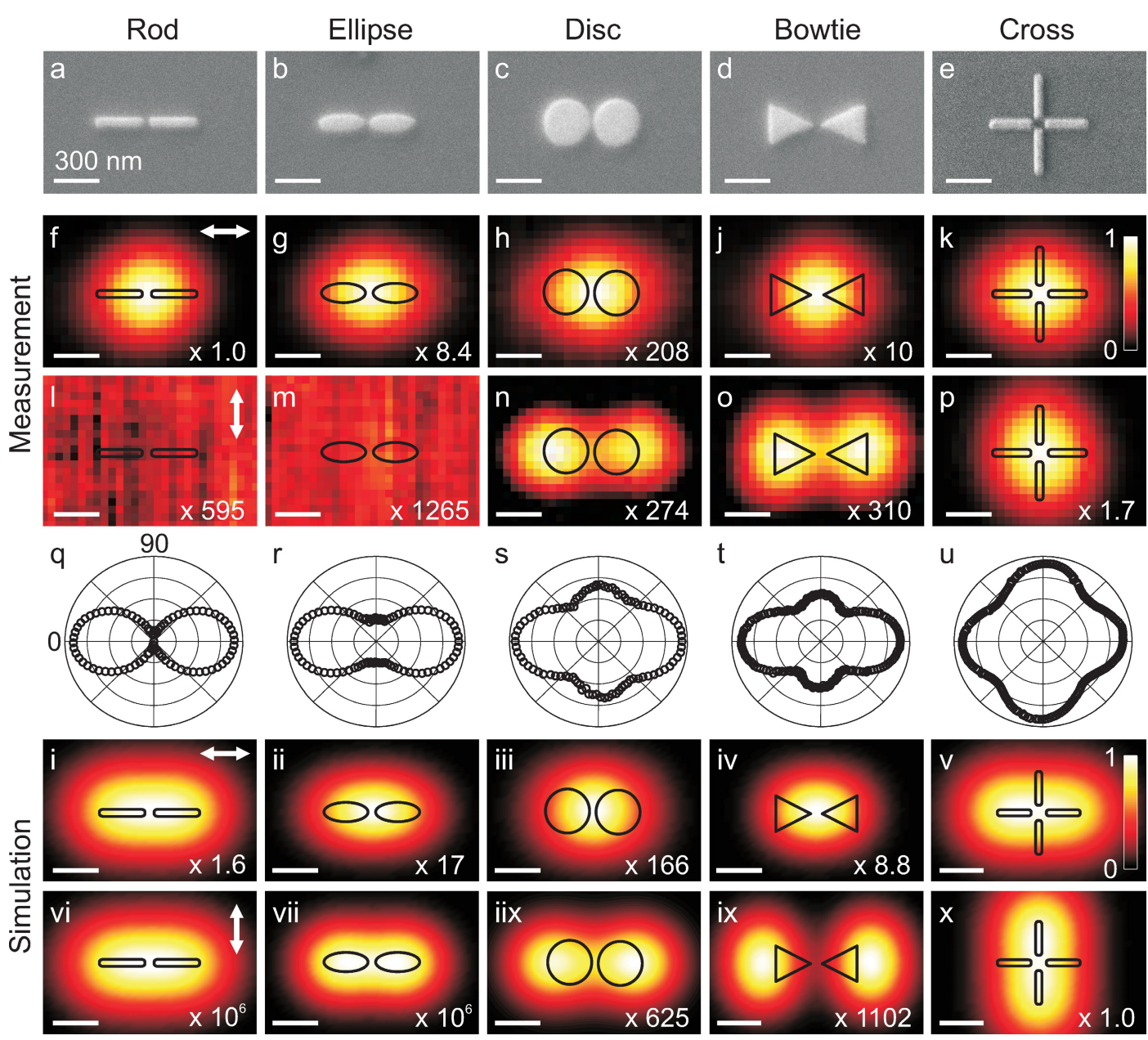

Figure 13.2.: Spatially resolved third-harmonic emission from five gold nanoantennas of different shape. $\mathbf{a}-\mathbf{e}$, Scanning electron microscope images. $\mathbf{f}-\mathbf{p}$, Measurement. The metal nanostructures are scanned through the focus of an oil immersion objective $(\mathrm{NA}=1.3)$ and excited by femtosecond laser pulses in the near infrared. Third-harmonic emission intensity is depicted in a normalized color scale as a function of position, recorded for horizontal $\mathrm{f}-\mathrm{k}$ and vertical $\mathrm{l}-\mathrm{p}$ linear polarization of the excitation. $\mathbf{q}-\mathbf{u}$, Polar plots of the third root of the measured third-harmonic intensity as a function of the polarization angle of the exciting beam. $\mathbf{i}-\mathbf{x}$, Boundary element simulations of the third-harmonic intensity for antenna geometries as in the experiment with horizontal $\mathrm{i}-\mathrm{v}$ and vertical $\mathrm{vi}-\mathrm{x}$ polarization of the excitation. All scale bars are $300 \mathrm{~nm}$. 
is raster-scanned within the confocal region with a step size of $50 \mathrm{~nm}$ and a dwell time of $50 \mathrm{~ms}$. Fig. $13.2 \mathrm{~g}-\mathrm{q}$ depict spatial maps of third-harmonic emission from single gold nanoantennas of different shapes. The free-space spatial resolution of our experiment is better than the lateral extension of the sub-wavelength antenna structures (Supplementary Information, Fig. 13.7). This performance is achieved using a third-order nonlinear process resulting in a much shorter wavelength for imaging as compared to the fundamental in the near infrared. It represents a distinct advantage over linear or lower-order optical methods [171,210] previously used to detect higher mode patterns of single nanoantennas. Here, we directly resolve the fundamental plasmonic mode which is accompanied by the highest field enhancement ( [61] and Supplementary Information, Fig. 13.8). We note that this technique is also ideal for rapid systematic investigations of nanoantennas. A typical high-resolution THG map with 600 pixels and a signal to noise ratio better than $10^{3}$ is recorded within 30 seconds. For linear polarization of the excitation set parallel to the resonant antenna axis ("horizontal") the third-harmonic emission maps for all types of nanoantennas (Fig. $13.2 \mathrm{f}-\mathrm{k}$ ) are found to exhibit the fundamental plasmon mode with a single hotspot in the gap region. For excitation polarized perpendicular to the main antenna axis ("vertical") neither the rod nor the elliptical nanoantenna fulfill the resonance condition for a near-infrared plasmon. As a consequence, the residual third-harmonic emission is at least three orders of magnitude weaker (Fig. $13.2 \mathrm{l}, \mathrm{m}$ ) compared to the horizontal configuration. The other three antenna shapes support plasmonic modes also in the vertical direction (Fig. $13.2 \mathrm{n}-\mathrm{p}$ ). The third-harmonic scans for the disc- and bowtie- shaped antennas are similar. A two-lobed pattern occurs due to the collective electron motion at the triangle bases and at the outer edges of the discs as a result of restoring Coulomb forces. The cross antenna features again one hotspot in the center. We note that arbitrary polarizations may be converted to the near field by this antenna type provided the gap size of the antenna is sufficiently small [219]. The dipolar behavior of the plasmonic modes is clearly seen in the polar plots (Fig. $13.2 \mathrm{q}-\mathrm{u}$ ) of the maximum signal as a function of the direction of incident linear polarization.

In order to quantitatively analyze these mode patterns we have carried out simulations of third-harmonic emission from single nanoantennas by using a boundary element method (BEM) [82,83]. The surface of the nanoantenna is discretized into 
triangles and the centroid of each triangle is taken as a collocation point where the electromagnetic fields are matched (see Supplementary Information, Fig. 13.5). We use the dielectric function of gold extracted from optical data [41] and a refractive index of 1.35 (weighted average of substrate and air) for the embedding medium. The spatial dimensions of the metal nanostructures are analogous to the experiment (Supplementary Information, Tab. 13.1 and Fig. 13.6). We consider a Gaussian excitation spot $(\mathrm{FWHM}=300 \mathrm{~nm})$ and take the third power of the electric field at the surface as source of the third-order polarizations. The coherent sum over these nonlinear dipoles then results in the third-harmonic signal. In the lower panel of Fig. $13.2 \mathrm{i}-\mathrm{x}$, we show the calculated THG maps. The theoretical results are almost perfectly in agreement with the corresponding experimental data above. Both, from experimental data and theoretical calculations of the spatial plasmonic patterns, we find that the fundamental mode is exclusively excited in all antenna types. Hence, a mechanism independent of the shape must be the reason for the different plasmon damping times and the pronounced changes in the emitted THG intensities.

To resolve this question, the basic antenna geometry is kept constant and only one antenna dimension is varied. Rod antennas with identical arm length $(s=260$ $\mathrm{nm})$, gap distance $(g=50 \mathrm{~nm})$ and thickness $(h=30 \mathrm{~nm})$ but varying rod widths are fabricated using electron beam lithography. Fig. 13.3 a shows the third-harmonic emission map of ten individual rod antennas. The nanodevices are spatially separated by $3 \mu \mathrm{m}$ from each other. The width of the antenna arms is increasing from $w=35$ $\mathrm{nm}$ on the left image side to $w=200 \mathrm{~nm}$ on the right. The inset of Fig. 13.3 depicts the narrowest and the widest antenna under investigation. The extracted maximum third-harmonic intensity as a function of rod width is plotted in Fig. 13.3 b. It is apparent that the THG intensity follows an inverse cubic dependence with the rod width identified by the straight line in the logarithmic plot of Fig. 13.3 b. The experimental observation highlights that high nonlinear light emission is directly connected to small antenna volumes. This observation strongly hints to radiative damping in these metal nanostructures, which is proportional to the number of coherently oscillating dipoles and therefore depending on the volume [220,221].

To prove this assumption we move back to the time domain and measure the plasmon damping times as a function of rod width of a second set of nanoantennas ( $s=150$ $\mathrm{nm}, h=20 \mathrm{~nm}, g=50 \mathrm{~nm}$ ). Although the variation in rod width causes a slight 


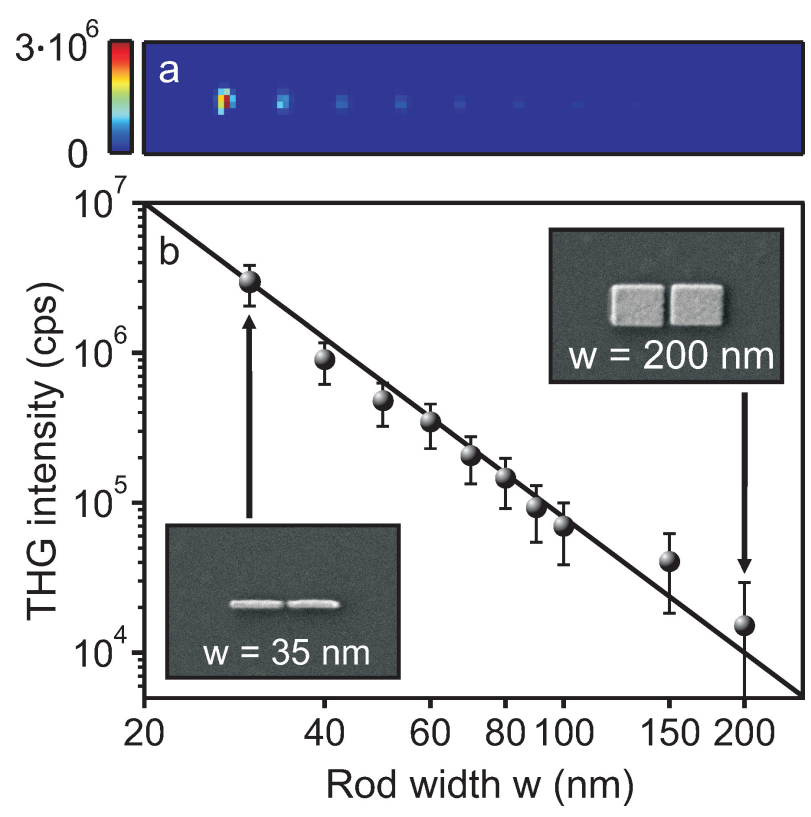

Figure 13.3.: Third-harmonic emission intensity of rod nanoantennas depending on their width. a, Color-coded third-harmonic emission scan of ten antennas with increasing width (from left to right). b. Extracted third harmonic emission intensity values (dots) together with an inverse cubic fit (black line) on a doubly logarithmic scale. Insets: Scanning electron microscope images of the narrowest (top left) and widest (bottom right) antenna, respectively. Arm length $l=260$ $\mathrm{nm}$, structure height $h=30 \mathrm{~nm}$, and gap distance $g=50 \mathrm{~nm}$ are kept constant.

change of the plasmon resonance $[146,222]$ (in our case the center wavelength shift of the third-harmonic is $22 \mathrm{~nm}$ ), resonant driving conditions are ensured through the large excitation bandwidth. We also verified that the pump power has no detectable effect on the damping times. The average pump power was minimized and adjusted between $0.2 \mathrm{~mW}$ and $0.6 \mathrm{~mW}$, keeping the third-harmonic intensities equally low for all antennas. As shown in Fig. 13.4, the plasmon damping time $\tau$ increases with decreasing antenna width. This damping of the fundamental plasmon oscillation directly corroborates the cubic dependence of the third order nonlinear emission on the inverse antenna volumes from Fig. 13.3. Simulated values for the damping times $\tau$ in Fig. 13.4 are gained by using the relation $\tau=\Gamma^{-1}$, where the homogeneous linewidth $\Gamma$ is extracted from scattering spectra calculated with the BEM method for exactly the same antenna geometries. The measured damping times quantitatively 


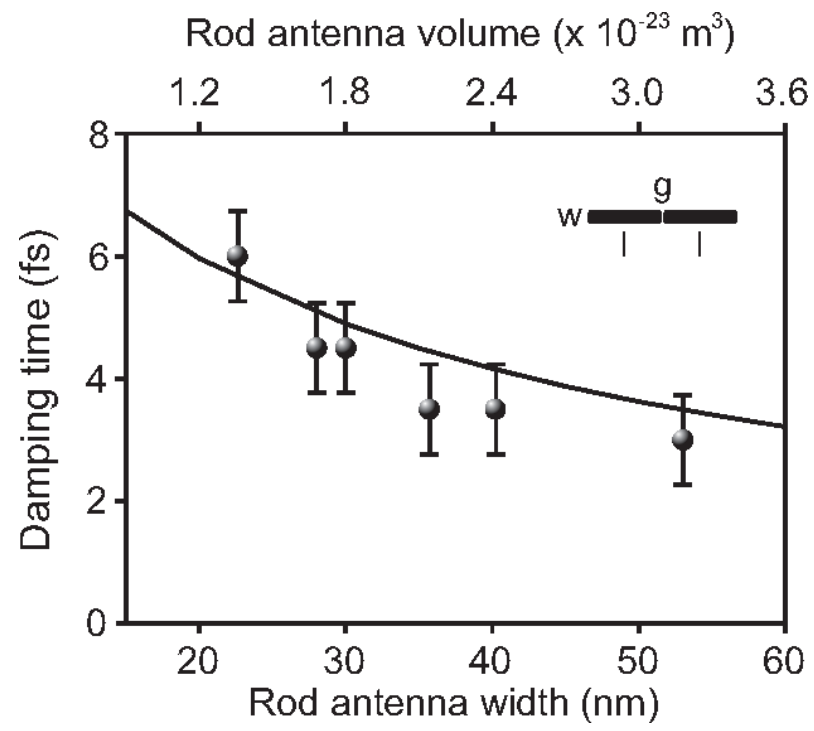

Figure 13.4.: Plasmon damping time of rod nanoantennas of different widths. Measured values are depicted as dots, while the simulated values are drawn as a solid line. Nanoantenna dimensions are: arm length $s=150 \mathrm{~nm}$, height $h=20$ $\mathrm{nm}$, and gap distance $g=50 \mathrm{~nm}$.

agree with simulated ones, which finally proves that our infrared nanoantennas with volumes between $1.4 \times 10^{-22} \mathrm{~m}^{3}$ and $3.4 \times 10^{-21} \mathrm{~m}^{3}$ are indeed dominated by radiative damping. Given that this is a purely dissipative process, we always discuss the energy relaxation time $\tau$. Under our conditions, $\tau$ is directly linked to the decoherence time $T_{2}$ of the oscillation amplitude via $T_{2}=2 \tau$ [223,224]. We would like to note that for gold nanorods with volumes below $0.6 \times 10^{-22} \mathrm{~m}^{3}$ and resonant in the adjacent visible range, damping was attributed to electronic interband transitions [135]. Only for small probed volumes and at higher plasmon energies, loss mechanisms such as carrier-carrier, carrier-lattice, and surface scattering become increasingly important $[225,226]$. In the near infrared, however, metal antennas are limited solely by the process they are intended for, namely radiation coupling.

In conclusion, we have presented nonlinear emission spectroscopy as a new powerful tool to characterize and design single metal nanoantennas in space and time. On the temporal scale, few-femtosecond plasmon damping times are directly measured for different antenna shapes. Due to radiative damping the lowest active volumes generate by far the strongest third-harmonic emission. On the spatial scale, 
the third-harmonic emission of different types of nanoantennas is mapped out to prove operation at the fundamental mode. By combining sub-wavelength concentration in space with few-cycle response times, the ultimate manipulation of light has been demonstrated in all four dimensions. Our findings provide design capabilities for nanoantennas with either an ultrabroadband spectral coverage or low radiative damping rates accompanied by maximum nonlinear efficiencies. This knowledge will also be decisive for the creation of tailored hybrid antenna-nanoemitter systems for applications in few-photon quantum optics on a femtosecond timescale [227]. 


\subsection{Supplementary Information}

(a)

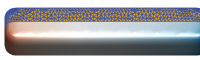

(c)

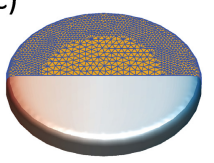

(b)
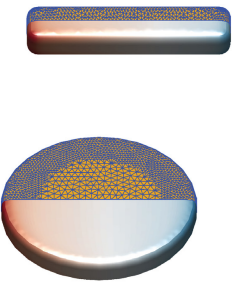

(d)
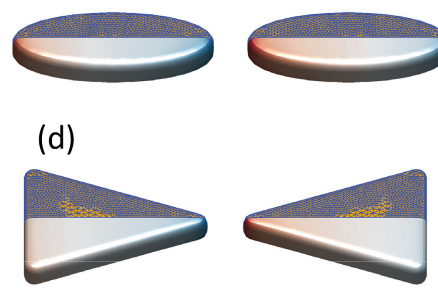

(e)
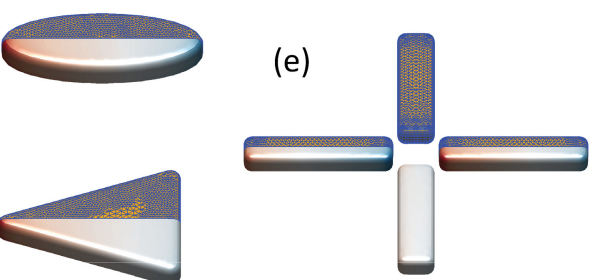

Figure 13.5.: Details of the simulation via the boundary element method. Surface discretization (upper halves of each plot) and real part of the normalized surface charges (lower halves) of the five investigated nanoantennas under resonant excitation with horizontal polarization. Together with surface currents they are used for solving Maxwell's equations [82,83]. The number of surface elements varies between 10000 and 20000. The dielectric function for gold is extracted from optical data [41] and a refractive index of 1.35 is adopted for the embedding medium (corresponding to a weighted average of fused silica substrate and air).

\begin{tabular}{lcccccccc}
\hline Antenna hape & Rod & Ellipse & Disc & Rod & Ellipse & Disc & Bowtie & Cross \\
Figure & $13.1 \mathrm{~d}$ & $13.1 \mathrm{c}$ & $13.1 \mathrm{~b}$ & $13.2 \mathrm{a}$ & $13.2 \mathrm{~b}$ & $13.2 \mathrm{c}$ & $13.2 \mathrm{~d}$ & $13.2 \mathrm{e}$ \\
\hline Arm length $l$ & 240 & 260 & 250 & 305 & 280 & 300 & 295 & 280 \\
Arm width $w$ & 45 & 100 & 250 & 60 & 110 & 300 & 240 & 285 \\
Height $h$ & 35 & 35 & 35 & 35 & 35 & 35 & 40 & 35 \\
Gap distance $g$ & 50 & 45 & 45 & 65 & 50 & 45 & 60 & 90 \\
\hline
\end{tabular}

Table 13.1.: Antenna dimensions in nm of Fig. 13.1 and 13.2 retrieved from scanning electron micrographs with an uncertainty of $5 \mathrm{~nm}$. Identical dimensions are used for the simulated nanostructures. 


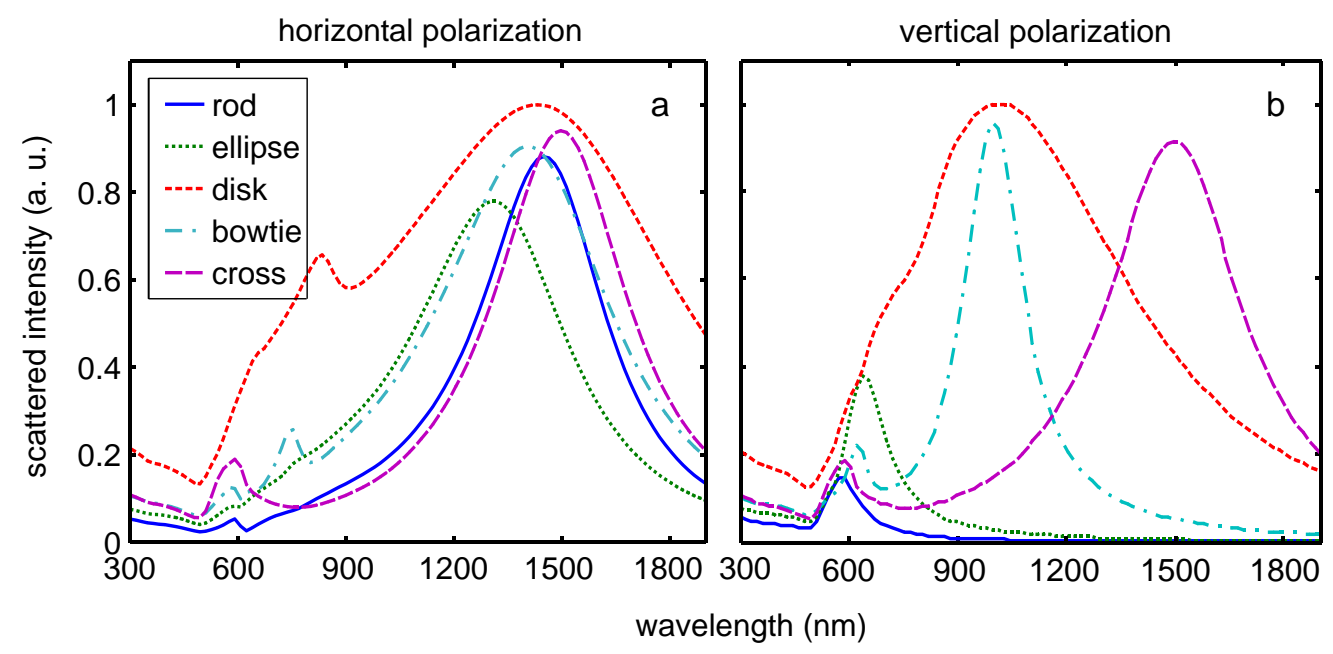

Figure 13.6.: Simulated scattering spectra for the five nanoantennas of Fig. 13.2 for horizontal $\mathbf{a}$ and vertical $\mathbf{b}$ linear polarization. While in the experiment the structures are optimized to yield maximum third-harmonic emission when driven in the wavelength range between $1.2 \mu \mathrm{m}$ and $1.5 \mu \mathrm{m}$, the nominal nanoparticle dimensions (see Tab. 13.1) are taken without further optimization in the simulation. As a result, the maxima of the emission peaks are varying. We account for this effect by setting the central frequency of the ultrabroadband femtosecond pulse resonant to the scattering maxima. 

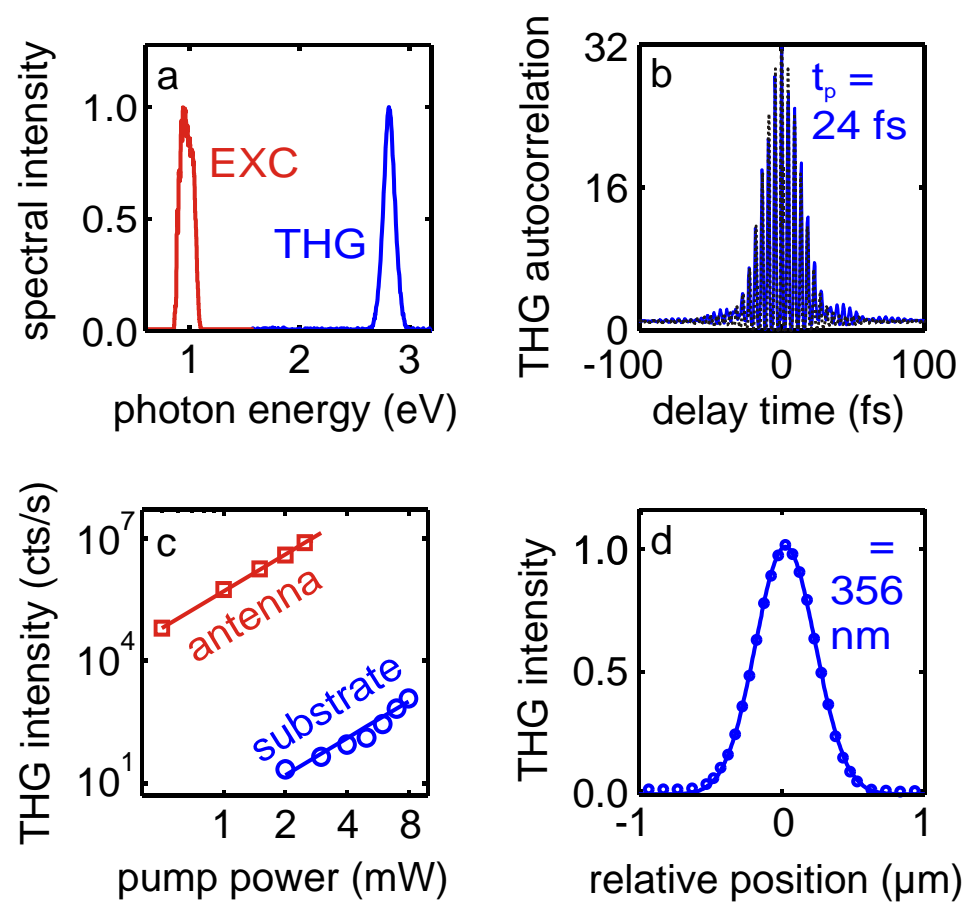

Figure 13.7.: Experimental details for third-harmonic imaging with sub-wavelength spatial resolution of Fig. 13.1 and 13.4. a, Excitation (red) and third-harmonic (blue) intensity spectrum. b, Measured (blue) and simulated (black) thirdharmonic intensity autocorrelation, retrieving a pulse duration of 24 fs. c, Pumppower dependence of the third-harmonic intensity for the fused silica substrate (blue) and a resonant rod antenna (red) together with cubic fits on a logarithmic scale. Note that the intensities differ by five orders of magnitude. d, Horizontal cut (blue dots) through the measured third-harmonic emission map of Fig. $13.1 \mathrm{i}$ together with a Gaussian fit (blue line), yielding a FWHM of $504 \mathrm{~nm}$. 
Fund. int. (cts/s) $0 \square 450$

a $=000+\ldots+3 \mu \mathrm{m}$

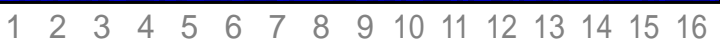

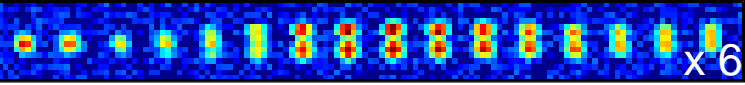

$1718192021222324252627282930 \quad 3132$

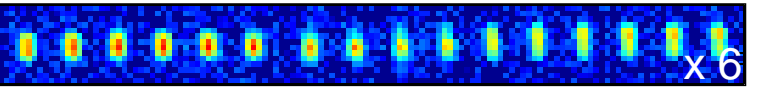

$33 \quad 3435 \quad 363738 \quad 3940 \quad 4142 \quad 4344 \quad 45 \quad 46 \quad 4748$

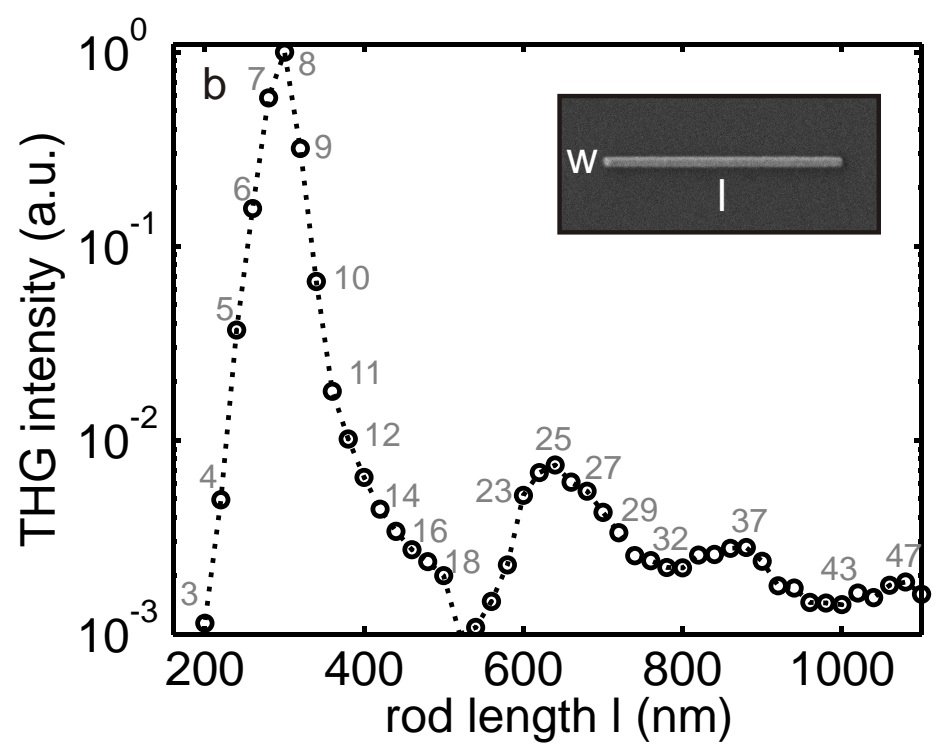

Figure 13.8.: Third-harmonic intensity of single gold nanorods on a fused silica substrate as a function of their length. a, Third root of the third-harmonic intensity scan (color coded) of 48 neighboring rods with rod length increasing from left to right and top to bottom. Note the successive appearance of higher resonances. b, Projection of the THG intensity values (black dots) to the corresponding rod lengths on a logarithmic scale. Despite the drastic THG intensity decrease we can identify up to the fourth mode. The inset shows a SEM image of one specimen with length $l=1.7 \mu \mathrm{m}$. The width $w=50 \mathrm{~nm}$ and thickness $t=30 \mathrm{~nm}$ of the nanoantennas are kept constant. 


\section{Part III.}

\section{Résumé}





\section{Summary}

\subsection{What has been done}

In this work we have discussed many aspects of the optical properties of metallic nanoparticles, ranging from the tunability of the plasmonic resonance to the ultrafast dynamics of the light-matter interaction. We have elaborated the theoretical description of metallic nanoparticles in terms of classical field theory, where the microscopic material response is lumped in the dielectric description. Besides the analytical solution for spheres and ellipsoids we have introduced the BEM and the DDA as versatile simulation tools for the numerical solution of the considered systems.

After the introductory chapters that should highlight the basics of plasmonic interactions and plasmon imaging, we presented a reprint of six papers, where we discussed the properties of an optimal plasmon sensor, the possibility to map the plasmonic LDOS with EELS and Moiré imaging, the influence of surface roughness and the effect of plasmon dephasing on the light confinement in single nanoantennas.

\subsection{What is left to do}

Our MNPBEM toolbox covers a broad area of simulation possibilities, but there is still plenty of room for improvements of course. For example a better interpolation scheme beyond the discussed collocation method as well as further code optimizations should be discussed. Another aspect that has not been addressed in this thesis is the influence of substrate effects, which, nevertheless, can be calculated with our toolbox. Besides the detailed testing of substrate effects we will also investigate grating effects, that come into play in the simulation of particle arrays and grids. 
One of the limitations listed in Sec. 4.5.3 is the possibility of charged particles, which occur in electrochemistry, for example. This is something which in principle can also be included within our MNPBEM toolbox, where the eigenmode expansion allows to incorporate an additional surface charge - but further investigation on this topic is also necessary.

Another very interesting topic that will keep us busy in the next time, is the possibility to generate holographic images of metallic nanoparticles with EELS [228]. This represents a very nice continuation of our EELS simulations and will hopefully allow deeper insights on the nonlocal interaction of high energetic electron beams with particle plasmons.

The key to success in any kind of fundamental research is to find the unexpected and forcing science into the corset of forecasted directions will not work. Thus the prediction of future perspectives in any kind of research field is inherently risky. Nevertheless because of the highly diverse nature of plasmonics we can assume an exciting future and high activity in this field as discussed in [229], where, for example, the merging of plasmonics with quantum systems, active plasmonic devices or biochemical applications are named as future trends.

There are still many other open questions to explore and we are looking forward to new challenges and tasks. 


\section{Acknowledgments}

When I finished my studies and got to decide which path on the scientific road I should take, I got the wise and simple advice to just trust my gut feeling. Well, this is what I did and the person who gave me this advice turned out to be a really great supervisor and a brilliant physicist. I want to thank Ulrich Hohenester for his guidance, his patience, for conveying his passion for physics and his directness. In his subtle way he constantly encouraged me and not even once I found his door closed when I was looking for his help and advice. He has a great talent to bring clearness into intricate problems and I'm still sometimes impressed with how quickly and efficiently he solves such difficulties.

Furthermore, I sincerely thank Joachim Kern, Alfred Leitner, Andreas Hohenau, Jean-Claude Tinguely, Daniel Koller and all other members of the Optical Nanotechnology / Nano-Optics group in Graz for most helpful discussions. I guess the straightforwardness and simplicity of our collaboration serves as a role model of fruitful teamwork which demonstrates how easily experimental and theoretical work can be enriched from each other.

I also want to express my gratitude for all my other collaborations, namely:

- the research group of Carsten Sönnichsen at the Institute for Physical Chemistry in Mainz, where Jan Becker and others did a marvelous job in putting our MATLAB $^{\circledR}$ toolbox to the acid test,

- the group of Alfred Leitenstorfer from the Lehrstuhl für Moderne Optik und Quantenelektronik - especially Tobias Hanke and Rudolf Bratschitsch (who changed to Chemnitz University of Technology in the meantime),

- the group of Ferdinand Hofer at the Austrian Centre for Electron Microscopy and Nanoanalysis (FELMI) in Graz, 
- Birgit Wiedemann and Michael Kaniber from the Walter Schottky institute (Center for Nanotechnology, Munich University of Technology), and

- Christoph Huber from the group of Wolfgang Kautek at the Institut für Physikalische Chemie in Vienna.

Special thanks also go to the theoretical and computational nanoscience group of Elisa Molinari in Modena for a great research stay at the beginning of my $\mathrm{PhD}$, where I was welcomed most cordially particularly by Rosa DiFelice and Guido Goldoni.

On a more personal basis, I would like to thank my family and friends. My parents supported me in every possible way, and no matter what difficulties there may come, my family will always bolster me. Without my two siblings, Thomas and Christine, life would be so much less fun and it is remarkable how different and how similar we are at the same time. It is really calming to know that there will always be a table large enough in my parents' house, where we all can come together.

Speaking of a heartily atmosphere, also every visit with Rosmarie and Thomas in Saalfelden leads to a replenishing of my batteries and when I see the rocky face of the Steinernes Meer it warms my heart - the difficulty of the plains are forgotten.

But above all I owe my deepest thankfulness to Steffi, for her love, her encouragement and for letting me see the real important things when sometimes the blinders of physics obscure my view.

I also want to thank Wolfgang, Markus, Daniela and Birgit, Gernot and Sabine, Gabi, Julia, Markus and Birgit, and all the others, who may forgive me for this incomplete list, for their heartfelt and deep friendship, and for accompanying me in my life. I also want to express my deep gratitude to Michi for our unforgettable trips to frozen waterfalls and glaciated mountain tops - feeding upon these memories keeps my head above water when every now and then the daily routine prevails. Special thanks also go to Jürgen (and his unshakable humor), who helped me to identify errors and typos in this work.

Last but not least I owe thanks to Richard Forsyth for many useful comments concerning the spelling in this thesis. In this respect, also the helpful remarks and advice of Margot Grösslich were of great help to me - thank you for that! 


\section{Part IV.}

\section{Supplement}





\section{A. Appendix - Utilities}

\section{A.1. Boundary conditions at different media}

By following [66], let $V$ be a finite space volume bordered by the surface area $\partial \Omega$, and let $\hat{\boldsymbol{n}}$ be the unit vector in outside direction of the surface element $d a$ (see Fig. A.1).

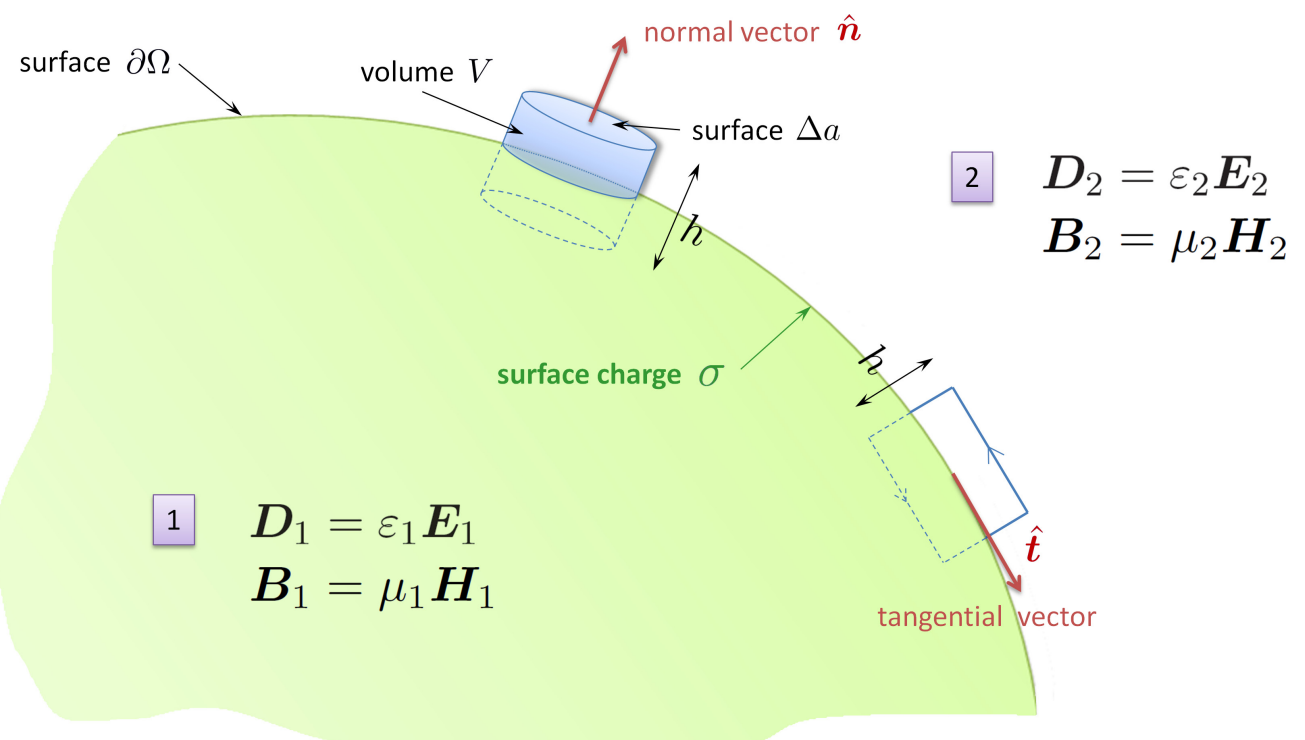

Figure A.1.: Schematic diagram of the interface between two different dielectric media 1 and 2, based on [66]. The small cylinder of height $h$ is given by the volume $V$ and surface $S$. The cylinder's lower and upper top surface area is labeled by $\Delta a$. The normal vector $\hat{\boldsymbol{n}}$ points in outside direction (from medium 1 to medium $2)$. The vector $\hat{\boldsymbol{t}}$ is tangential to the surface $\partial \Omega$. The surface spanned by the rectangular kink $C$ is perpendicular to the interface, i.e., $\hat{\boldsymbol{t}}$ is perpendicular to the kink. At the interface $\partial \Omega$ exists an idealized surface charge $\sigma$. 
At first we transform Eq. (3.1a) and Eq. (3.1b) to their corresponding integral form and apply them to the volume of the small cylinder in Fig. A.1. In the limit of infinitesimal height $(h \rightarrow 0)$ only the lower and upper top surface area are non-zero. If we assume that this surface has the value $\Delta a$ and approximately set $\boldsymbol{D}_{1}$ and $\boldsymbol{D}_{2}$ constant within the surface element, it follows that

$$
\begin{aligned}
& \oint_{S} \mathbf{D} \cdot \mathbf{n} d a=\left(\mathbf{D}_{2}-\mathbf{D}_{1}\right) \cdot \mathbf{n} \Delta a \\
& \oint_{S} \mathbf{B} \cdot \mathbf{n} d a=\left(\mathbf{B}_{2}-\mathbf{B}_{1}\right) \cdot \mathbf{n} \Delta a
\end{aligned}
$$

If the charge density $\varrho$ at the surface $\partial \Omega$ is singular and forms an idealised surface charge density $\sigma$, the right hand side of Eq. (3.1a) yields

$$
4 \pi \int_{V} \rho d^{3} x=4 \pi \sigma \Delta a
$$

From these equations follow the boundary conditions for the normal components of $\boldsymbol{D}$ and $\boldsymbol{B}$ :

$$
\begin{aligned}
\left(\mathbf{D}_{2}-\mathbf{D}_{1}\right) \cdot \boldsymbol{n} & =4 \pi \sigma \\
\left(\mathbf{B}_{2}-\mathbf{B}_{1}\right) \cdot \boldsymbol{n} & =0
\end{aligned}
$$

Applying a similar procedure to the rectangular kink $C$ with (3.1c) and (3.1d), using Stoke's theorem yields the boundary conditions for the tangential field components:

$$
\begin{gathered}
\boldsymbol{n} \times\left(\mathbf{E}_{2}-\mathbf{E}_{1}\right)=0, \\
\boldsymbol{n} \times\left(\mathbf{H}_{2}-\mathbf{H}_{1}\right)=\boldsymbol{j},
\end{gathered}
$$

where $j$ is an idealized surface current density.

For the quasistatic case the scalar potential $\Phi$ obeys analogous conditions, which are 
quickly derived by using the relations

$$
\boldsymbol{D}_{1}=\varepsilon_{1} \boldsymbol{E}_{1}=-\varepsilon_{1} \nabla \Phi_{1}, \quad \boldsymbol{D}_{2}=\varepsilon_{2} \boldsymbol{E}_{2}=-\varepsilon_{2} \boldsymbol{\nabla} \Phi_{2} .
$$

Inserting this in Eq. (A.4) leads to

$$
\left.\varepsilon_{1} \Phi_{1}^{\prime}\right|_{\text {surf }}=\left.\varepsilon_{2} \Phi_{2}^{\prime}\right|_{\text {surf }}
$$

where the apostrophe ' denotes the normal derivative, i.e. the derivative in a direction perpendicular to the surface. Eq. (A.6) shows, that the tangential components of $\boldsymbol{E}$ are continuous at the interface and therefore the derivation of the potential in tangential direction is also continuous.

Using spherical coordinates and considering a sphere with radius $a$ and surface charge density $\sigma$ in a dielectric medium yields

$$
\begin{gathered}
\left.\varepsilon_{\text {in }} \frac{\partial \Phi_{\text {in }}}{\partial r}\right|_{a}-\left.\varepsilon_{\text {out }} \frac{\partial \Phi_{\text {out }}}{\partial r}\right|_{a}=4 \pi \sigma \\
\left.\frac{\partial \Phi_{\text {in }}}{\partial \theta}\right|_{a}-\left.\frac{\partial \Phi_{\text {out }}}{\partial \theta}\right|_{a}=0 .
\end{gathered}
$$




\section{A.2. Conversion between $\mathrm{nm}$ and $\mathrm{eV}$}

The energy of a photon is given by

$$
E=\hbar \omega=\hbar 2 \pi \nu=\hbar 2 \pi \frac{c}{\lambda} .
$$

The SI-values for the involved constants can be found in [230], for example, and are given by

$$
\begin{array}{ll}
\hbar=6.58211899 \times 10^{-16} & {[\mathrm{eV} \mathrm{s}],} \\
c=2.99792458 \times 10^{17} & {[\mathrm{~nm} / \mathrm{s}] .}
\end{array}
$$

From Eq. (A.11) then follows ${ }^{1}$

Conversion from $\mathrm{eV}$ to $\mathrm{nm}$

$$
[\mathrm{eV}]=1239.84 /[\mathrm{nm}]
$$

The values of the visible spectrum of light in $\mathrm{eV}$ and $\mathrm{nm}$ are shown in Fig. A.2.

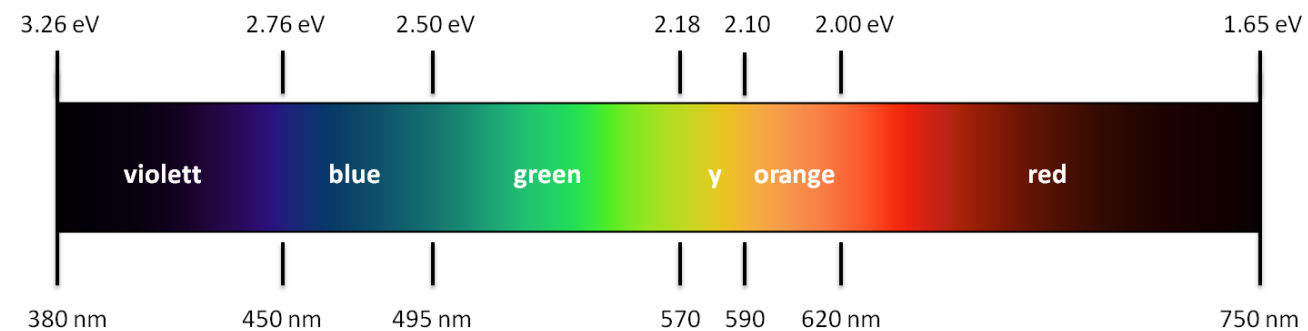

Figure A.2.: Energy values of the visible spectrum in $\mathrm{nm}$ and $\mathrm{eV}$, the approximated color ranges were taken from [231]. The range from $750-3000 \mathrm{~nm}(1.65-0.41 \mathrm{eV})$ is called near-infrared, the region from $10-380 \mathrm{~nm}(123.98-3.26 \mathrm{eV})$ ultraviolet [231].

\footnotetext{
${ }^{1}$ In [43] also the value $8065.48\left[\mathrm{~cm}^{-1} / \mathrm{ev}\right]$ is published.
} 


\section{A.3. Conversion between FWHM and decay time}

By following [42], let us investigate the damped oscillation of an electric field $E(t)$. The exponential decay shall be described by a time constant $\tau$, the eigenfrequency by $\omega_{0}$. From the Fourier transform of $E(t)$

$$
\begin{aligned}
E(t) & =E_{0} e^{-i \omega_{0} t} e^{-t / \tau}, \\
\mathcal{F}\{E(t)\} & =\frac{E_{0}}{\sqrt{2 \pi}} \frac{1 / \tau}{\left(\omega-\omega_{0}\right)^{2}+(1 / \tau)^{2}},
\end{aligned}
$$

follows a so called Lorentz-lineshape (see Fig. A.3), which is characterized by its center position $\omega_{0}$ and the full width at half maximum (FWHM) $\Delta \omega$. In units of energy the FWHM is given by $\Gamma=\hbar \Delta \omega$. By determining the extrema it follows that half of the maximum for Eq. (A.16) is reached at $\left(\omega-\omega_{0}\right)=1 / \tau$, which directly leads to the connection of the FWHM with the decay time: $\Gamma=2 \hbar / \tau$. With Eq. (A.12) and Eq. (A.14) we finally obtain

\section{Conversion between FWHM and decay time}

$$
\tau[\mathrm{fs}]=\frac{1.316424}{\Gamma[\mathrm{eV}]} \quad \text { or } \quad \tau[\mathrm{fs}]=1.061767 \times 10^{-3} \beta,
$$

where we have used the abbreviation

$$
\beta=\left(\frac{1}{\lambda_{1}}-\frac{1}{\lambda_{2}}\right)^{-1}=\frac{\lambda_{1} \lambda_{2}}{\lambda_{2}-\lambda_{1}}
$$

Here $\lambda_{1}$ and $\lambda_{2}$ correspond to the left and right limits of the FWHM (see Fig. 2.21), respectively.

The same relation also holds for a driven harmonic oscillator, see [42] for further details. Note that the oscillator energy (amplitude squared) decays twice as fast as the oscillation amplitude! 

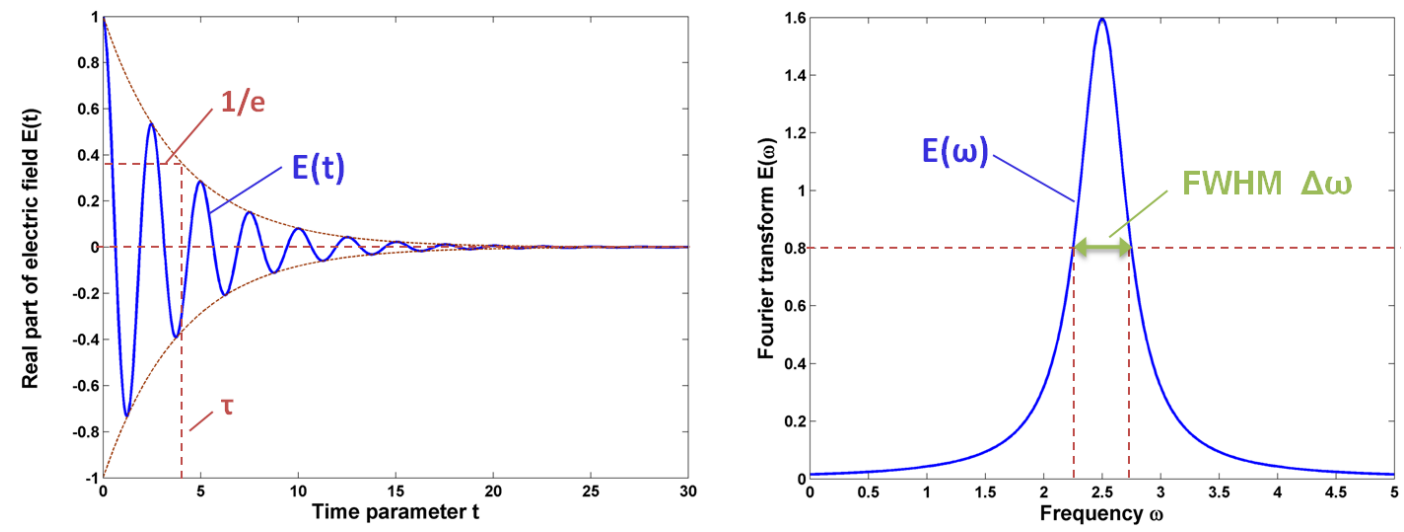

Figure A.3.: Lorentzian curve (right panel, Eq. (A.16)) obtained by the Fourier transform of a damped oscillator in the time domain (left panel, Eq. (A.15)) [42]. Chosen parameters: Damping time $\tau=4$, frequency $\omega_{0}=2.5$. The FWHM is $\Delta \omega$ $=0.5$, which reproduces the damping time: $2 / \Delta \omega=4$ 


\section{A.4. Derivation of retarded surface charges and currents}

The continuity of the tangential magnetic field follows as

$$
H_{1} \boldsymbol{h}_{1}-H_{2} \boldsymbol{h}_{2}-i k \hat{\boldsymbol{n}}\left(G_{1} \varepsilon_{1} \sigma_{1}-G_{2} \varepsilon_{2} \sigma_{2}\right)=\Delta \boldsymbol{A}_{\mathrm{ext}}^{\prime}+i k \hat{\boldsymbol{n}}\left(\varepsilon_{1} \Phi_{1}-\varepsilon_{2} \Phi_{2}\right)
$$

where $H_{1,2}=F \pm 2 \pi \mathbb{1}$. The continuity of the normal electric displacement gives

$$
\begin{aligned}
& H_{1} \varepsilon_{1} \sigma_{1}-H_{2} \varepsilon_{2} \sigma_{2}-i k \hat{\boldsymbol{n}} \cdot\left(G_{1} \varepsilon_{1} \boldsymbol{h}_{1}-G_{1} \varepsilon_{1} \boldsymbol{h}_{2}\right)= \\
& \varepsilon_{1}\left(i k \hat{\boldsymbol{n}} \cdot \boldsymbol{A}_{1}^{\mathrm{ext}}-\Phi_{1}^{\prime}\right)-\varepsilon_{2}\left(i k \hat{\boldsymbol{n}} \cdot \boldsymbol{A}_{2}^{\mathrm{ext}}-\Phi_{2}^{\prime}\right) .
\end{aligned}
$$

We now insert Eq. (3.42) into Eq. (A.18) and Eq. (A.19) and use

$$
\begin{aligned}
& H_{1} \boldsymbol{h}_{1}=H_{1} G_{1}^{-1}\left(G_{2} \boldsymbol{h}_{2}+\Delta \boldsymbol{A}_{\mathrm{ext}}\right)=\Sigma_{1}\left(G_{2} \boldsymbol{h}_{2}+\Delta \boldsymbol{A}_{\mathrm{ext}}\right), \\
& H_{2} \boldsymbol{h}_{2}=H_{2} G_{2}^{-1}\left(G_{2} \boldsymbol{h}_{2}\right)=\Sigma_{2}\left(G_{2} \boldsymbol{h}_{2}\right) .
\end{aligned}
$$

where $\Sigma_{1,2}=H_{1,2} G_{1,2}^{-1}$. Furthermore we get $H_{1} \varepsilon_{1} \sigma_{1}=\Sigma_{1} L_{1} \sigma_{1}$, where $L_{1,2}=$ $G_{1,2} \varepsilon_{1,2} G_{1,2}^{-1}$. After some more algebra we obtain

$$
\begin{gathered}
\left(\begin{array}{ll}
\left.\Sigma_{1}-\Sigma_{2} \quad\right) G_{2} \boldsymbol{h}_{2}-i k \hat{\boldsymbol{n}}\left(L_{1}-L_{2}\right) G_{2} \sigma_{2}= \\
\Delta \boldsymbol{A}_{\mathrm{ext}}^{\prime}+i k \hat{\boldsymbol{n}}\left(\varepsilon_{1} \Phi_{1}-\varepsilon_{2} \Phi_{2}\right)-\Sigma_{1} \Delta \boldsymbol{A}_{\mathrm{ext}}+i k \hat{\boldsymbol{n}} L_{1} \Delta \phi_{\mathrm{ext}}
\end{array}\right. \\
\left(\Sigma_{1} L_{1}-\Sigma_{2} L_{2}\right) G_{2} \sigma_{2}-i k \hat{\boldsymbol{n}}\left(L_{1}-L_{2}\right) G_{2} \boldsymbol{h}_{2}= \\
D_{e}^{\prime}-\Sigma_{1} L_{1} \Delta \phi_{\mathrm{ext}}+i k \hat{\boldsymbol{n}} \cdot L_{1} \Delta \boldsymbol{A}_{\mathrm{ext}} .
\end{gathered}
$$

For a compact notation we introduce the following abbreviations:

$$
\begin{aligned}
D_{e} & =D_{e}^{\prime}-\Sigma^{-1} L_{1} \Delta \phi_{\mathrm{ext}}+i k \hat{\boldsymbol{n}} \cdot L_{1} \Delta \boldsymbol{A}_{\mathrm{ext}}, \\
D_{e}^{\prime} & =\varepsilon_{1}\left(i k \hat{\boldsymbol{n}} \cdot \boldsymbol{A}_{1}^{\mathrm{ext}}-\Phi_{1}^{\prime}\right)-\varepsilon_{2}\left(i k \hat{\boldsymbol{n}} \cdot \boldsymbol{A}_{2}^{\mathrm{ext}}-\Phi_{2}^{\prime}\right), \\
\vec{\alpha} & =\vec{\alpha}^{\prime}-\Sigma_{1} \Delta \boldsymbol{A}_{\mathrm{ext}}+i k \hat{\boldsymbol{n}} L_{1} \Delta \phi_{\mathrm{ext}}, \\
\vec{\alpha}^{\prime} & =\Delta \boldsymbol{A}_{\mathrm{ext}}^{\prime}+i k \hat{\boldsymbol{n}}\left(\varepsilon_{1} \phi_{1}-\varepsilon_{2} \phi_{2}\right), \\
\Sigma & =\Sigma_{1} L_{1}-\Sigma_{2} L_{2}+k^{2} \hat{\boldsymbol{n}} \cdot\left(L_{1}-L_{2}\right) \Delta^{-1} \hat{\boldsymbol{n}}\left(L_{1}-L_{2}\right), \text { and } \Delta=\Sigma_{1}-\Sigma_{2} .
\end{aligned}
$$




\section{A.5. Spherical harmonics}

Many problems in physics lead to equations containing the Laplace operator $\triangle$, e.g. the wave or the Poisson equation. Depending on the symmetry of the considered problem one will try to solve the equations with a suitable set of variables. If spherical symmetry is present, one will choose the spherical coordinates $(r, \theta, \varphi)$. For example the Laplace equation

$$
\triangle f(r, \theta, \varphi)=0
$$

can be solved by the ansatz $f(r, \theta, \varphi)=R(r) S(\theta) T(\phi)$ (see e.g. [232]). The Laplace operator in this coordinate system reads

$$
\triangle \equiv\left[\frac{1}{r^{2}} \frac{\partial}{\partial r}\left(r^{2} \frac{\partial}{\partial r}\right)+\frac{1}{r^{2} \sin \theta} \frac{\partial}{\partial \theta}\left(\sin \theta \frac{\partial}{\partial \theta}\right)+\frac{1}{r^{2} \sin ^{2} \theta} \frac{\partial^{2}}{\partial \varphi^{2}}\right]
$$

and the above product ansatz allows us to separate Eq. (A.22) into three parts. The expression for $\varphi$ yields a wave equation with the solutions $\left\{e^{i m \varphi}, e^{-i m \varphi}\right\}$, and the one for the variable $\theta$ has the form of an associated Legendre differential equation with the associated Legendre polynomials $P_{l}^{m}(\cos \theta)[233]$ as solution. Therefore the angular part of the complete solution is given by

$$
T(\varphi) S(\theta)=e^{ \pm i m \varphi} P_{l}^{m}(\cos \theta)
$$

This leads to the definition of an orthogonal system of functions, and together with a normalization factor ${ }^{2}$ the spherical harmonics $Y_{l m}[233]$ are derived $^{3}$ :

$$
Y_{l m}(\theta, \varphi)=\sqrt{\frac{(2 l+1)}{4 \pi} \frac{(l-m) !}{(l+m) !}} P_{l}^{m}(\cos \theta) e^{i m \varphi}
$$

\footnotetext{
${ }^{2}$ Unfortunately also different normalization factors and signs can be found in the literature. We will stick to the definition (A.25).

${ }^{3}$ Another less obvious but more elegant way is the definition of spherical harmonics as components of some irreducible tensor of rank $l$ with the commutation relations $\left[L_{\mu}, Y_{l m}(\theta, \varphi)\right]=$ $\sqrt{l(l+1)} C_{l m-\mu}^{l m+\mu} Y_{l m+\mu}(\theta, \varphi)$ where $L_{\mu}(\theta, \varphi)$ is a spherical component of the operator $\mathbf{L}$. See [234] for further details.
} 
A detailed explanation and rigorous definition of these functions can be found in the book of Varshalovich et al. [234], which we will follow from here on.

In Eq. (A.25) we see that a spherical harmonic $Y_{l m}(\theta, \varphi)$ is a single-valued, continuous, bounded complex function of two real arguments $\theta, \varphi$ with $0 \leq \theta \leq \pi$ and $0 \leq \phi<2 \pi$. For a given $l$ there exist $(2 l+1)$ functions corresponding to different $m$ 's.

These functions are eigenfunctions of the orbital angular momentum operator in quantum mechanics which is defined as $(\hbar=1)$ [235]

$$
\mathbf{L} \equiv \mathbf{r} \times \mathbf{p} \equiv \frac{1}{i}(\mathbf{r} \times \boldsymbol{\nabla}) .
$$

The eigenvalue equations read

$$
\begin{aligned}
& \mathbf{L}^{2} Y_{l m}(\theta, \varphi)=l(l+1) Y_{l m}(\theta, \varphi), \\
& L_{z} Y_{l m}(\theta, \varphi)=m Y_{l m}(\theta, \varphi),
\end{aligned}
$$

or in expanded form

$$
\begin{gathered}
{\left[\frac{1}{\sin \theta} \frac{\partial}{\partial \theta}\left(\sin \theta \frac{\partial}{\partial \theta}\right)+\frac{1}{\sin ^{2} \theta} \frac{\partial^{2}}{\partial \varphi^{2}}+l(l+1)\right] Y_{l m}(\theta, \varphi)=0} \\
{\left[i \frac{\partial}{\partial \varphi}+m\right] Y_{l m}(\theta, \varphi)=0}
\end{gathered}
$$

In Eq. (A.27) one can see that $l$ specifies the absolute value of orbital angular momentum (because $l(l+1)$ is the eigenvalue of $\mathbf{L}^{2}$ ) and $m$ in Eq. (A.28) is the eigenvalue of $L_{z}$, which is the projection of the orbital angular momentum operator on the quantisation axis.

Equation (A.29) has two linearly independent solutions for fixed $l$ and $m$, but only one of them is regular (i.e. satisfies the condition $\left|Y_{l m}(\theta, \varphi)\right|^{2}<\infty$ ) while the other is singular at $\theta=0$ and $\theta=\pi$. In quantum mechanics and electrodynamics the regular solution is of major interest. The homogeneous boundary conditions

$$
\begin{gathered}
Y_{l m}(\theta, \varphi \pm 2 \pi n)=Y_{l m}(\theta, \varphi), \\
\left.\frac{\partial}{\partial \varphi} Y_{l m}(\theta, \varphi)\right|_{\theta=0, \pi}=0,
\end{gathered}
$$


lead to integer values of $l$ and $m$ (with $|m| \leq l$ ). Since the differential equations (A.29) and (A.30) and the boundary conditions determine the spherical harmonics only up to some arbitrary complex factor, a normalisation of the functions is needed.

\section{A.5.1. Completeness and normalisation}

The normalisation and orthogonality relation of spherical harmonics [233] is given by

$$
\int_{0}^{2 \pi} d \varphi \int_{0}^{\pi} d \theta \sin \theta Y_{l m}^{*}(\theta, \varphi) Y_{l^{\prime} m^{\prime}}(\theta, \varphi)=\delta_{l l^{\prime}} \delta_{m m^{\prime}},
$$

and the completeness relation reads as follows:

$$
\sum_{l=0}^{\infty} \sum_{m=-l}^{+l} Y_{l m}^{*}(\theta, \varphi) Y_{l m}\left(\theta^{\prime}, \varphi^{\prime}\right)=\delta\left(\varphi-\varphi^{\prime}\right) \delta\left(\cos \theta-\cos \theta^{\prime}\right)
$$

A detailed list of symmetry properties can again be found in [234]. Here we only mention the complex conjugation

$$
Y_{l m}^{*}(\theta, \varphi)=Y_{l m}(\theta,-\varphi)=(-1)^{m} Y_{l-m}(\theta, \varphi) .
$$

\section{A.5.2. Expansions in series of the spherical harmonics}

An arbitrary function $f(\theta, \varphi)$ which is defined in the interval $0 \leq \theta \leq \pi, 0 \leq \varphi<2 \pi$ and satisfies the condition

$$
\int_{0}^{2 \pi} d \varphi \int_{0}^{\pi} d \theta \sin \theta|f(\theta, \varphi)|^{2}<\infty
$$

can be expanded into a series of spherical harmonics as

$$
f(\theta, \varphi)=\sum_{l=0}^{\infty} \sum_{m=-l}^{l} a_{l m} Y_{l m}(\theta, \varphi),
$$


with the expansion coefficients $a_{l m}$ given by

$$
a_{l m}=\int_{0}^{2 \pi} d \varphi \int_{0}^{\pi} d \theta \sin \theta Y_{l m}^{*}(\theta, \varphi) f(\theta, \varphi) .
$$

This last relation may be considered as an integral transformation of $f(\theta, \varphi)$ from the continuous variables $\theta, \varphi$ to the discrete variables $l, m$. The transformation matrix in this case is given by $Y_{l m}(\theta, \varphi) \equiv\langle\theta, \varphi \mid l m\rangle^{4}$ :

$$
\langle l m \mid f\rangle=\langle l m \mid \theta, \varphi\rangle\langle\theta, \varphi \mid f\rangle,
$$

where

$$
\langle l m \mid f\rangle \equiv a_{l m}, \quad\langle\operatorname{lm} \mid \theta, \varphi\rangle \equiv Y_{l m}^{*}(\theta, \varphi), \quad\langle\theta, \varphi \mid f\rangle \equiv f(\theta, \varphi)
$$

The expansion (A.37) is widely used in different branches of physics. It is called the multipole expansion and $a_{l m}$ are the multipole moments (e.g. see [66]). The transformations (A.38) and (A.39), respectively, are unitary

$$
\langle f \mid l m\rangle\langle l m \mid f\rangle=\langle f \mid \theta, \varphi\rangle\langle\theta, \varphi \mid f\rangle,
$$

and the expansion coefficients $a_{l m}$ satisfy the Parseval condition (see [233] or [232])

$$
\sum_{l=0}^{\infty} \sum_{m=-l}^{+l}\left|a_{l m}\right|^{2}=\int_{0}^{2 \pi} d \varphi \int_{0}^{\pi} d \theta \sin \theta|f(\theta, \varphi)|^{2}
$$

\footnotetext{
$\overline{{ }^{4} \text { Summation or integration is assumed }}$ over all variables which are repeated twice.
} 


\section{A.6. Generating functions for the vector harmonics}

In Sec. 3.2 and Eq. (3.16) we see, that the time-harmonic electromagnetic field in a linear, isotropic, homogeneous medium fulfills a wave equation:

$$
\left(\nabla^{2}+\varepsilon \frac{\omega^{2}}{c^{2}}\right)\left\{\begin{array}{l}
\boldsymbol{E}(\boldsymbol{r}, \omega) \\
\boldsymbol{B}(\boldsymbol{r}, \omega)
\end{array}\right\}=0 .
$$

By following [93] let us now construct a vector $\mathcal{M}$ with a given scalar function $\psi$ and an arbitrary constant vector $\boldsymbol{c}$ :

$$
\mathcal{M}=\nabla \times(c \psi)
$$

Note that this vector is always perpendicular to $\boldsymbol{c}: \boldsymbol{\mathcal { M }}=-\boldsymbol{c} \times \boldsymbol{\nabla} \psi$. Since the divergence of the curl of any vector function vanishes, we immediately get

$$
\nabla \cdot \mathcal{M}=0
$$

If we insert $\mathcal{M}$ into the wave equation and use the following identities

$$
\begin{aligned}
\nabla \times(\boldsymbol{A} \times \boldsymbol{B}) & =\boldsymbol{A}(\boldsymbol{\nabla} \cdot \boldsymbol{B})-\boldsymbol{B}(\boldsymbol{\nabla} \cdot \boldsymbol{A})+(\boldsymbol{B} \cdot \boldsymbol{\nabla}) \boldsymbol{A}-(\boldsymbol{A} \cdot \boldsymbol{\nabla}) \boldsymbol{B} \\
\boldsymbol{\nabla}(\boldsymbol{A} \cdot \boldsymbol{B}) & =\boldsymbol{A} \times(\boldsymbol{\nabla} \times \boldsymbol{B})+\boldsymbol{B} \times(\boldsymbol{\nabla} \times \boldsymbol{A})+(\boldsymbol{B} \cdot \boldsymbol{\nabla}) \boldsymbol{A}+(\boldsymbol{A} \cdot \boldsymbol{\nabla}) \boldsymbol{B},
\end{aligned}
$$

we see that

$$
\boldsymbol{\nabla}^{2} \mathcal{M}+k^{2} \mathcal{M}=\boldsymbol{\nabla} \times\left[\boldsymbol{c}\left(\nabla^{2} \psi+k^{2} \psi\right)\right] .
$$

If now $\psi$ is a solution to the scalar wave equation

$$
\nabla^{2} \psi+k^{2} \psi=0
$$

$\mathcal{M}$ automatically fulfills the vector wave equation. 


\section{B. Appendix - Fabrication methods}

One of the reasons for the huge increase of research activities of plasmonic nanoparticles in the last decades roots in the improvement of nanofabrication methods and in the involved advancement in the control of matter at the nanoscale. Two main techniques are widely used for the fabrication of metallic nanoparticles: Chemically synthesis and electron beam lithography together with lift-off based vapor deposition. For the sake of completeness, in the following we briefly introduce these two techniques.

\section{B.1. Chemical synthesis}

A widely-used seeded-growth technique for chemical synthetisation of gold nanoparticles was proposed by Nikobaakht et al. in [151], also see [236]. With their approach, rod-shaped particles are grown in a two step method [237]. First a gold salt is quickly reduced in an aqueous medium to elementary gold in metallic form, resulting in nanospheres with an added organic molecule as shell to prevent their aggregation. During the second part a small amount of the previous dispersion is added to a solution containing gold salt in a slower reductive medium, promoting the reaction to metallic gold in the surface of the gold nanospheres. With the organic molecule shell presenting the tendency to bind better to specific crystal orientations, the growth of the particles in a specific direction is favored leading to rod-like, singlecrystalline structures. Lengths for a nanorod synthesized through this route can be varied between $50 \mathrm{~nm}$ and few nanometers, with 25-30 $\mathrm{nm}$ breadth, relatively small size deviations achievable with small aspect ratios.

Nanoparticles made of silver can be obtained by reducing silver nitrate with ethylene glycol in the presence of a water-soluble polymer and sodium sulfide as described 
in $[53,238]$. The particle shape can be controlled by the temperature and the reaction time (with the original method from [238] silver nanocubes are fabricated, applying a higher temperature and a longer reaction time gives rod-shaped particles [53]). Silver triangles for example can be synthesized by a photo-induced process, where spherical silver colloids are transformed into triangular nanoplates [53,239].

\section{B.2. Electron beam lithography}

In the following Fig. B.1 the principle process steps of nanoparticle fabrication with electron beam lithography are shown, see [240].

(1) cleaned substrate surface with ITO

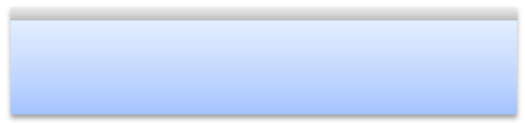

(3) writing nanostructure with e-beam

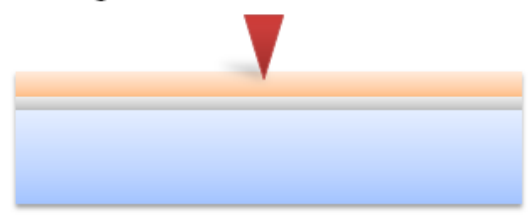

(5) high vacuum metal evaporation

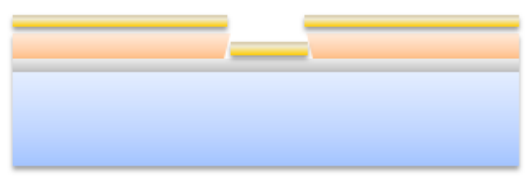

(2) spin-coating PMMA layer

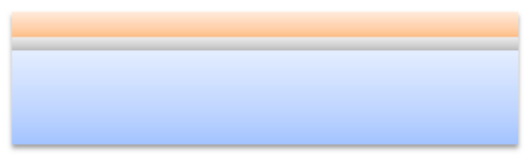

(4) chemical developement

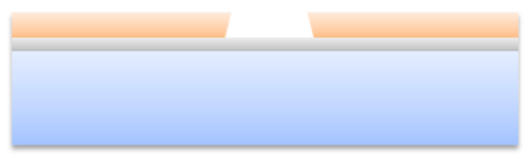

(6) lift off and cleaning

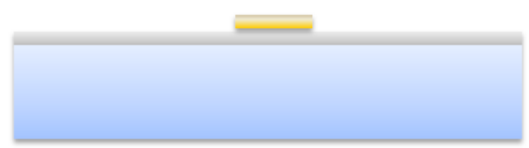

Figure B.1.: Fabrication steps of electron beam lithography as described in the text, figure based on [240].

1. One starts with a cleaned substrate surface, whose material is chosen accordingly to the wanted application. If the material is non-conductive (like a glass substrate), a conductive layer of indium-tin oxide (ITO) or metal has to be deposited on the substrate (high vacuum vapor deposition). 
2. In a spin-coat process a typically $100 \mathrm{~nm}$ thick layer of PMMA is put on the structure. This layer is a positive electron resist and has to be baked on a heating plate at $170{ }^{\circ} \mathrm{C}$ for 8 hours [240].

3. The PMMA layer is exposed by an electron beam and the desired nanoparticle structure is written.

4. After the electron beam exposure the resist layer is chemically developed. (Starting with a developer bath for $30 \mathrm{~s}$, immediately followed by a chemical stopper bath for $30 \mathrm{~s}$ and an isopropanol rinsing step [240].)

5. After cleaning, the nanoparticle metal (e.g. gold) is deposited in a high vacuum evaporation process.

6. An acetone bath is used to remove the remaining PMMA layer resist.

7. To finish the fabrication process, the surface is cleaned with a final isopropanol rinsing.

The resulting particles are polycrystalline and their surface roughness is higher than for chemical synthesized particles (also see Chap. 6). Thermal annealing could help to reduce the roughness and leads to reduced damping, an increasing absorption and the FWHM becomes narrower, see [237]. 



\section{Appendix - MATLAB}

\section{C.1. MATLAB memory limitations for different operating systems}

\begin{tabular}{|c|c|}
\hline Operating system and software platform & Process Limit \\
\hline 32-bit Windows 7, XP and Vista & $2 \mathrm{~GB}$ \\
\hline 32-bit Windows XP, Vista with 3 GB switch & $3 \mathrm{~GB}$ \\
\hline 32-bit Linux & $\sim 3 \mathrm{~GB}$ \\
\hline 64-bit Operating system running 32-bit MATLAB ${ }^{\oplus}$ & $\leq 4 \mathrm{~GB}$ \\
\hline 64-bit Operating system running 64-bit MATLAB ${ }^{\oplus}$ & $8 \mathrm{~TB}$ \\
\hline
\end{tabular}

Table C.1.: Memory characteristics and limitations for different MATLAB ${ }^{\circledR}$ platforms and operating systems. The process limit is the maximum amount of virtual memory a single process (or application) can address - on 32-bit systems, this is the most important factor limiting data set size. The MATLAB ${ }^{\circledR}$ memory function allows to verify the current process limit on Windows systems. Data and table adopted from MATLAB ${ }^{\circledast}$ Product Support 1107, Avoiding 'Out of Memory' Errors, http://www . mathworks. com/support/tech-notes/1100/1107.html. 


\begin{tabular}{|c|c|c|c|c|}
\hline $\begin{array}{c}\text { Operating } \\
\text { system and } \\
\text { configuration }\end{array}$ & $\begin{array}{c}\text { Total workspace } \\
\text { size }\end{array}$ & $\begin{array}{l}\text { Largest matrix } \\
\text { size }\end{array}$ & $\begin{array}{l}\text { Number of } \\
\text { Elements in } \\
\text { largest real } \\
\text { double array }\end{array}$ & $\begin{array}{l}\text { Number of } \\
\text { elements in } \\
\text { largest int } 8 \\
\text { array }\end{array}$ \\
\hline $\begin{array}{c}\text { 32-bit Windows } \\
\text { XP }\end{array}$ & $\sim 1700 \mathrm{MB}$ & $\sim 1189 \mathrm{MB}$ & $\sim 155 \times 10^{6}$ & $\sim 1246 \times 10^{6}$ \\
\hline $\begin{array}{c}\text { 32-bit Windows } \\
\text { Vista }\end{array}$ & $\sim 1643 \mathrm{MB}$ & $\sim 1428 \mathrm{MB}$ & $\sim 187 \times 10^{6}$ & $\sim 1497 \times 10^{6}$ \\
\hline $\begin{array}{l}\text { 32-bit Windows } \\
\text { XP, best case, } \\
\text { with } 3 \text { GB switch }\end{array}$ & $\sim 2700 \mathrm{MB}$ & $\sim 1536 \mathrm{MB}$ & $\sim 200 \times 10^{6}$ & $\sim 1610 \times 10^{6}$ \\
\hline 32-bit Linux & $\sim 2683 \mathrm{MB}$ & $\sim 2385 \mathrm{MB}$ & $\sim 312 \times 10^{6}$ & $\begin{array}{c}2^{31}-2 \\
\left(\sim 2147 \times 10^{6}\right)\end{array}$ \\
\hline $\begin{array}{c}\text { MAC OS X } \\
\text { running 32-bit } \\
\text { MATLAB }^{\circledR}\end{array}$ & $\sim 2919 \mathrm{MB}$ & $\sim 1532 \mathrm{MB}$ & $\sim 200 \times 10^{6}$ & $\sim 1606 \times 10^{6}$ \\
\hline $\begin{array}{l}\text { 64-bit Windows } \\
\text { XP running } \\
\text { 32-bit MATLAB }\end{array}$ & $\sim 3155 \mathrm{MB}$ & $\sim 2047 \mathrm{MB}$ & $\sim 268 \times 10^{6}$ & $\begin{array}{c}2^{31}-2 \\
\left(\sim 2147 \times 10^{6}\right)\end{array}$ \\
\hline $\begin{array}{c}\text { 64-bit Linux } \\
\text { running 32-bit } \\
\text { MATLAB }^{\circledR}\end{array}$ & $\sim 3558 \mathrm{MB}$ & $\sim 2292 \mathrm{MB}$ & $\sim 300 \times 10^{6}$ & $\begin{array}{c}2^{31}-2 \\
\left(\sim 2147 \times 10^{6}\right)\end{array}$ \\
\hline $\begin{array}{l}\text { Solaris running } \\
\text { 32-bit MATLAB }\end{array}$ & $\sim 3535 \mathrm{MB}$ & $\sim 3072 \mathrm{MB}$ & $\sim 402 \times 10^{6}$ & $\begin{array}{c}2^{31}-2 \\
\left(\sim 2147 \times 10^{6}\right)\end{array}$ \\
\hline $\begin{array}{c}\text { 64-bit OS } \\
\text { running } 64 \text {-bit } \\
\text { MATLAB }{ }^{\circledast} 7.4 \\
\text { and earlier }\end{array}$ & $\sim<8 \mathrm{~TB}$ & $\begin{array}{c}16 \mathrm{~GB} \text { (double } \\
\text { array) / 2GB } \\
\text { (int8 array) }\end{array}$ & $\begin{array}{c}2^{31}-2 \\
\left(\sim 2147 \times 10^{6}\right)\end{array}$ & $\begin{array}{c}2^{31}-2 \\
\left(\sim 2147 \times 10^{6}\right)\end{array}$ \\
\hline $\begin{array}{l}\text { 64-bit OS } \\
\text { running } 64 \text {-bit } \\
\text { MATLAB } \\
\text { and later }\end{array}$ & $\sim<8 \mathrm{~TB}$ & $<8 \mathrm{~TB}$ & $\begin{array}{c}2^{48}-1 \\
\left(\sim 2.8 \times 10^{14}\right)\end{array}$ & $\begin{array}{c}2^{48}-1 \\
\left(\sim 2.8 \times 10^{14}\right)\end{array}$ \\
\hline
\end{tabular}

Table C.2.: Largest (real double) matrix and total workspace size for different platforms. Table adopted from MATlAB ${ }^{\circledast}$ Product Support 1110, Maximum Matrix Size by Platform, http://www.mathworks.com/support/tech-notes/1100/ 1110.html. 


\section{C.2. MATLAB script for Mie solution}

With the following code example the scattering, absorption and extinction cross section for a spherical particle of arbitrary shape can be calculated. The dielectric data for the sphere has to be provided, viz. [41] $]^{1}$.

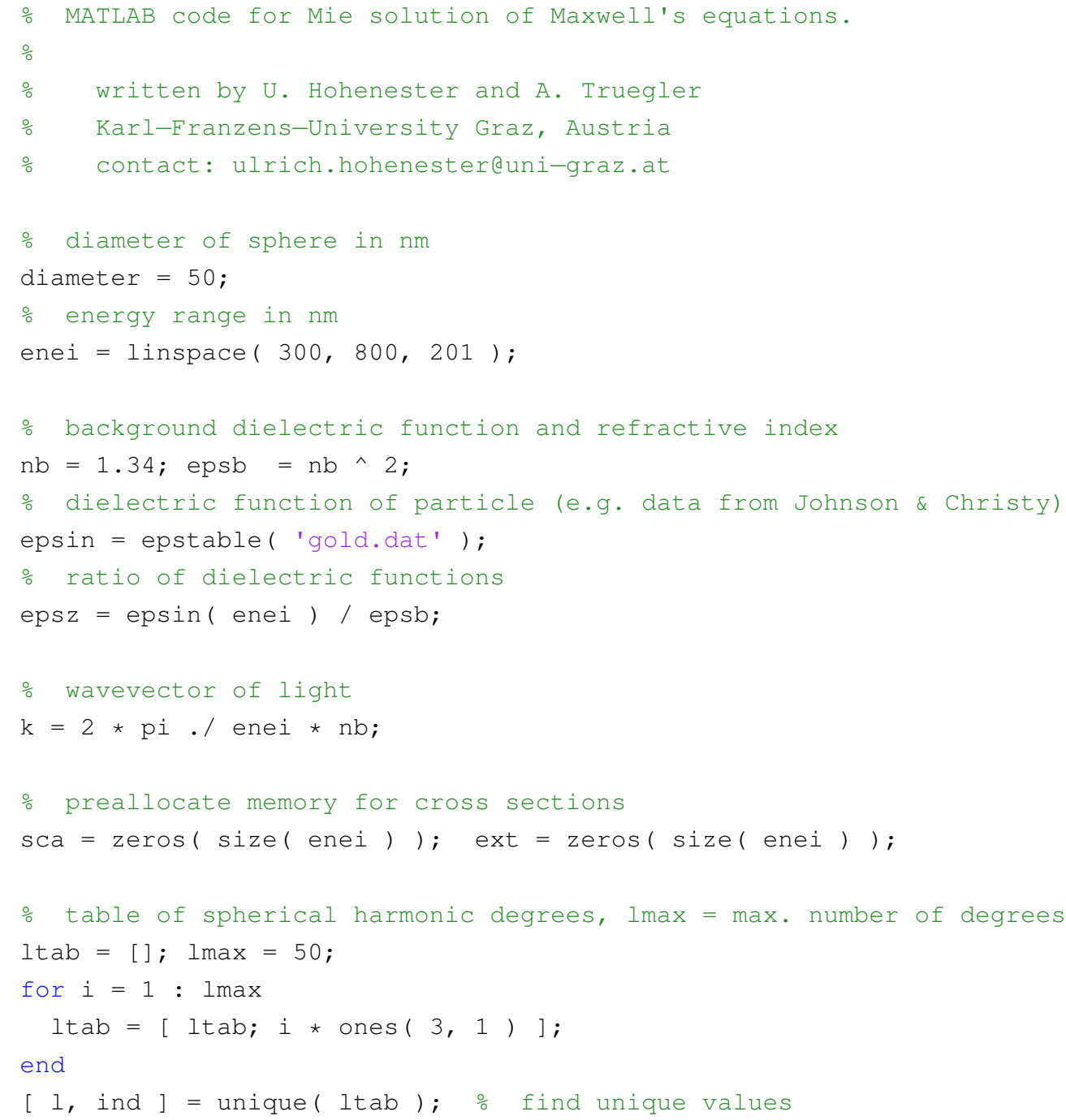

${ }^{1}$ Remember that usually the real and imaginary part of the refractive index $n=\tilde{n}+i \tilde{k}$ is given and therefore $\Re e\{\varepsilon\}=\tilde{n}^{2}-\tilde{k}^{2}$ and $\Im m\{\varepsilon\}=2 \tilde{n} \tilde{k}$. 


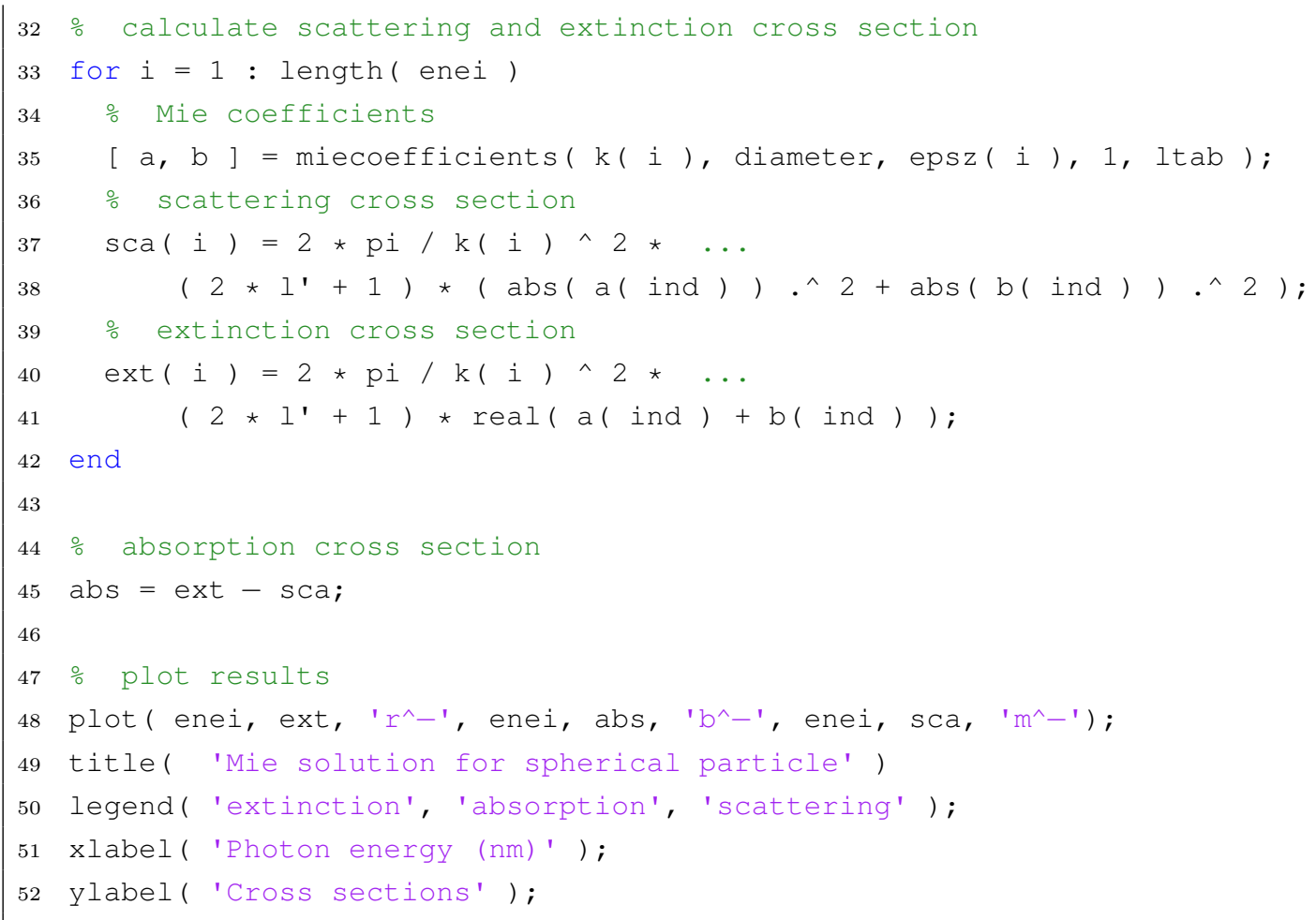

The function miecoefficients.m calculates the Mie coefficients of Eq. (4.10).

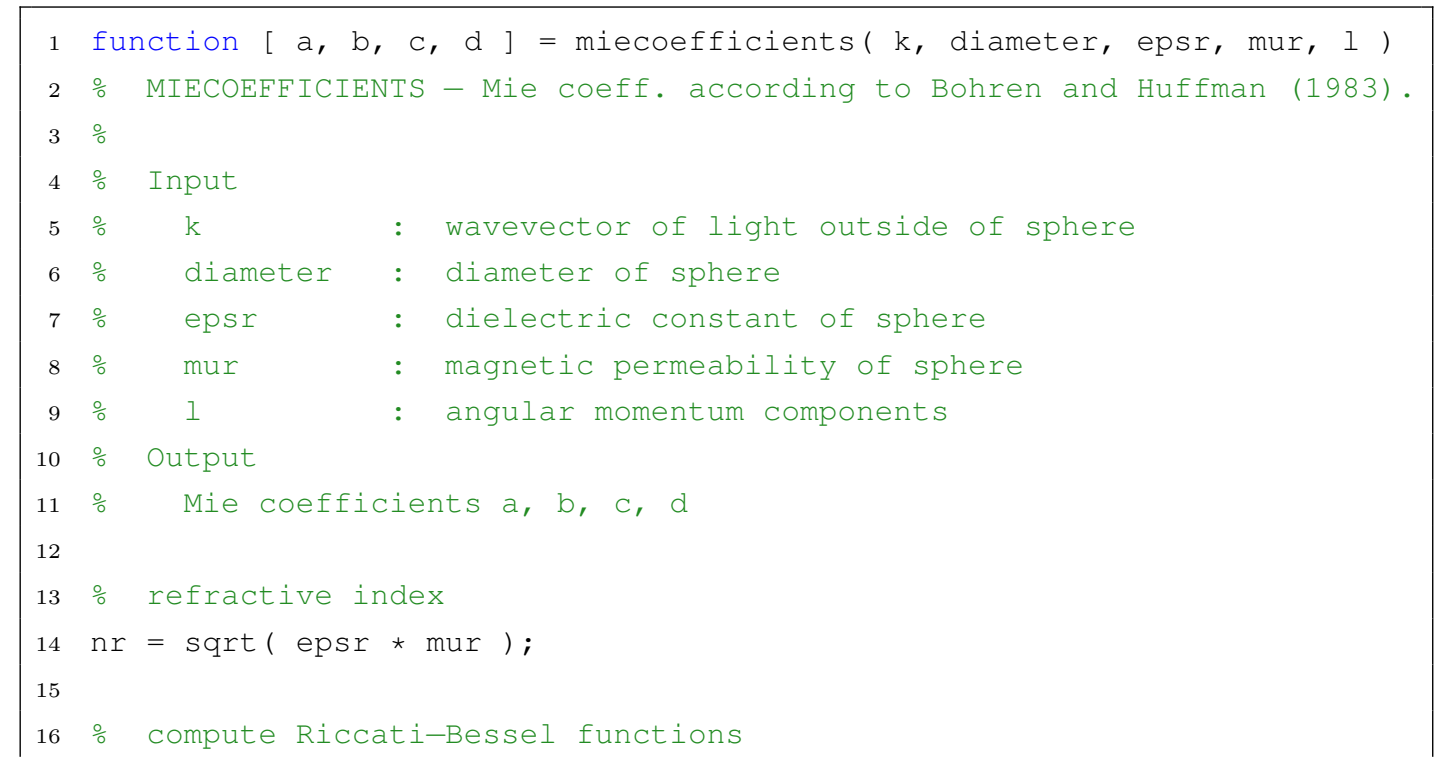




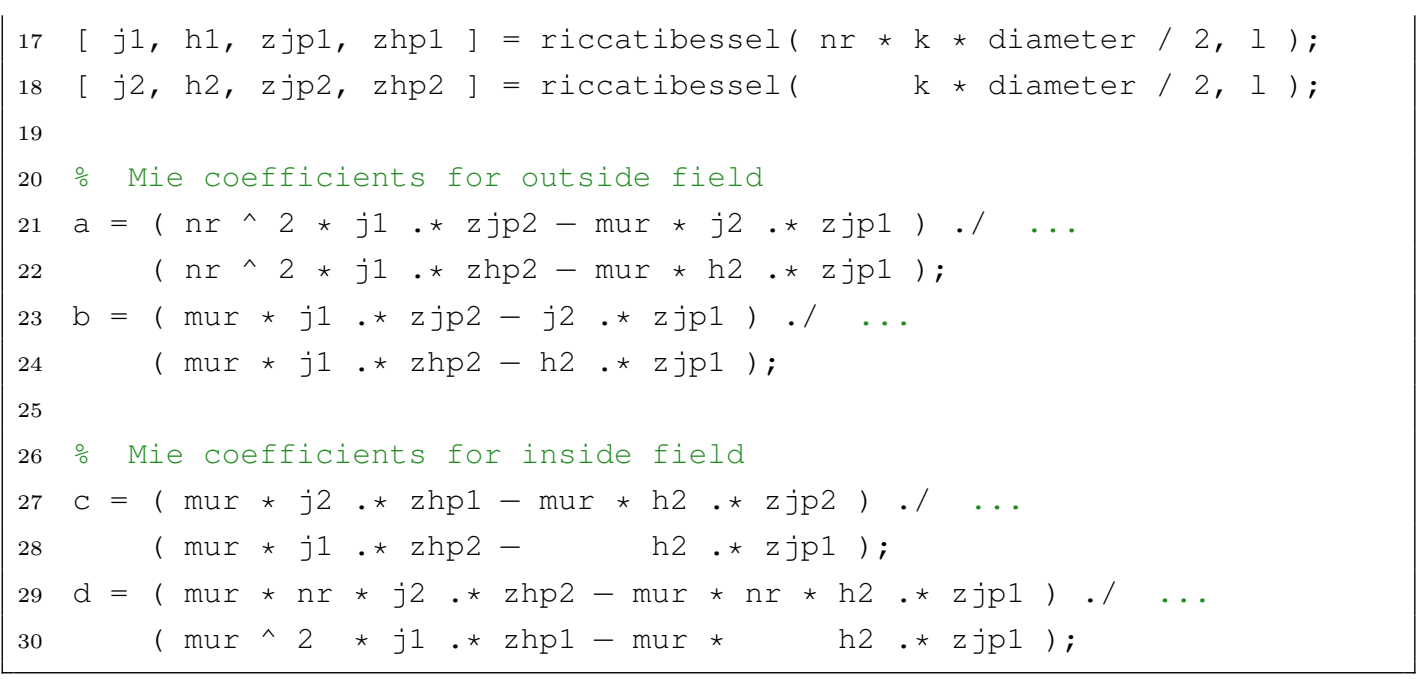

The function riccatibessel.m provides the spherical Bessel functions, see Eq. (4.11).

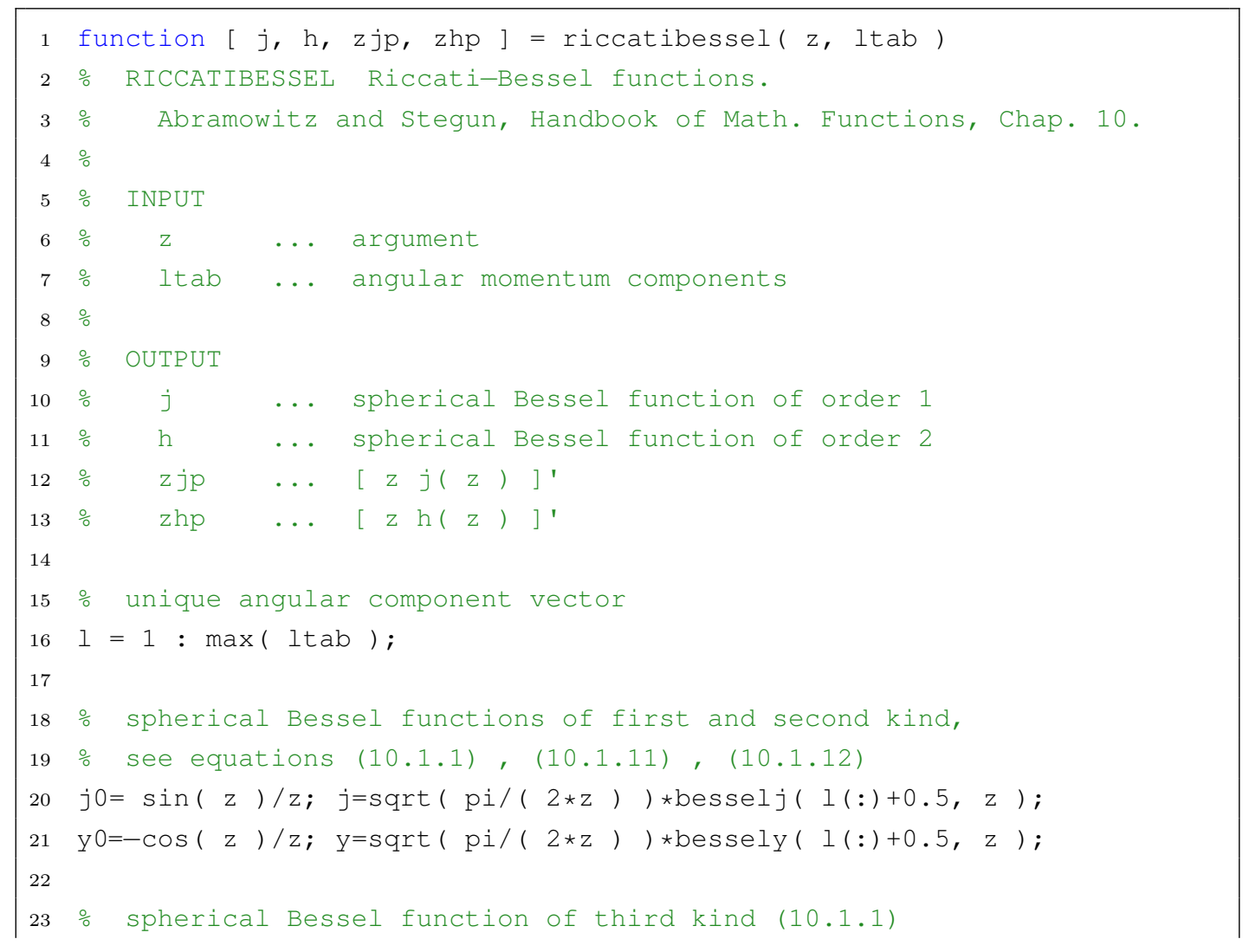




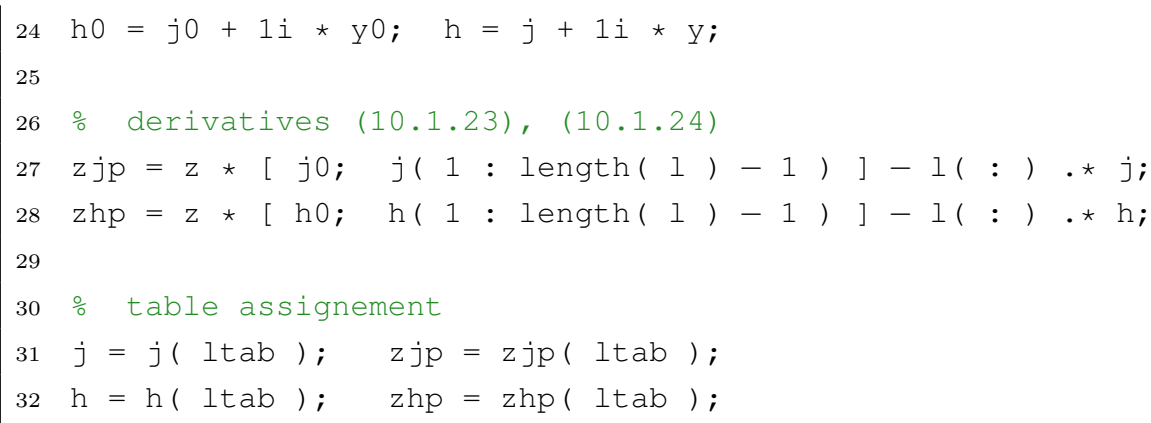




\section{Bibliography}

[1] R. Feynman, "There is plenty of room at the bottom (talk tanscript)," Caltech Engineering and Science 23:5 (1960) 22-36.

[2] N. Taniguchi, ed., On the Basic Concept of Nano-Technology, Proceedings of the International Conference on Production Engineering. Japan Society of Precision Engineering, Tokyo, 1974. Part II.

[3] K. E. Drexler, Molecular Machinery and Manufacturing with Applications to Computation. PhD thesis, Massachusetts Institute of Technology (MIT), Cambridge, Massachusetts, 1991.

[4] L. Novotny and B. Hecht, Principles of Nano-Optics. Cambrigde University Press, UK, 2006, ISBN 978-0521832243.

[5] H. Atwater, "The promise of plasmonics," Scientific American 296(4) (2007) 56.

[6] I. H. El-Sayed, X. Huang, and M. A. El-Sayed, "Selective laser photo-thermal therapy of epithelial carcinoma using anti-EGFR antibody conjugated gold nanoparticles," Cancer Lett. 239 (2006) 129-135.

[7] J. N. Anker, W. P. Hall, O. Lyandres, N. C. Shah, J. Zhao, and R. P. V. Duyne, "Biosensing with plasmonic nanosensors," Nat. Mater. 7 (2008) 442.

[8] A. Haes and R. P. V. Duyne, "A Nanoscale Optical Biosensor: Sensitivity and Selectivity of an Approach Based on the Localized Surface Plasmon Resonance Spectroscopy of Triangular Silver Nanoparticles," J. Am. Chem. Soc. 124 (2002) 10596.

[9] P. Fortina, L. J. Kricka, D. J. Graves, J. Park, T. Hyslop, F. Tam, N. Halas, S. Surrey, and S. A. Waldman, "Applications of nanoparticles to diagnostics and therapeutics in colorectal silver nanoparticles," JACS 124 (2007) 10596.

[10] J. B. Pendry, "Negative refraction makes a perfect lense," Phys. Rev. Lett. 85 (2000) 3966. 
[11] H. J. Lezec, J. A. Dionne, and H. A. Atwater, "Negative refraction at visible frequencies," Science 316 (2007) 5823.

[12] N. I. Zheludev, "A Roadmap for Metamaterials," Opt. Photonics News 22 (2011) 30-35.

[13] P. Zijlstra, J. M. Chon, and M. Gu, "Five-dimensional optical recording mediated by surface plasmons in gold nanorods," Nat. 459 (2009) 410-413.

[14] B. Lamprecht, J. R. Krenn, G. Schider, H. Ditlbacher, M. Salerno, N. Felidj, A. Leitner, F. R. Aussenegg, and J. C. Weeber, "Surface plasmon propagation in microscale metal stripes," Appl. Phys. Lett. 79 (2001) 51.

[15] S. I. Bozhevolnyi, V. S. Volkov, E. Deveaux, J. Y. Laluet, and T. W. Ebbensen, "Channel plasmon subwavelength waveguide components including interferometers and ring resonators," Nature 440 (2006) 509.

[16] A. Akimov, A. Mukherjee, C. L. Yu, D. E. Chang, A. S. Zibrov, P. R. Hemmer, H. Park, and M. D. Lukin, "Generation of single optical plasmons in metallic nanowires coupled to quantum dots," Nat. 450 (2007) 402-406.

[17] R. Kolesov, B. Grotz, G. Balasubramanian, R. J. Stöhr, A. A. L. Nicolet, P. R. Hemmer, F. Jelezko, and J. Wachtrup, "Wave-particle duality of single surface plasmon polaritons," Nat. Phys. 5 (2009) 470-474.

[18] D. M. Koller, A. Hohenau, H. Ditlbacher, N. Galler, F. Reil, F. R. Aussenegg, A. Leitner, E. J. W. List, and J. R. Krenn, "Organic plasmon-emitting diode," Nat. Phot. 2 (2008) 684-687.

[19] A. L. Falk, F. H. L. Koppens, C. L. Yu, K. Kang, N. de Leon Snapp, A. V. Akimov, M.-H. Jo, M. D. Lukin, and H. Park, "Near-field electrical detection of optical plasmons and single-plasmon sources," Nat. Phys. 5 (2009) 475-479.

[20] V. E. Ferry, L. A. Sweatlock, D. Pacifici, and H. A. Atwater, "Plasmonic Nanostructure Design for Efficient Light Coupling into Solar Cells," Nano Lett. 8 (2008) 4391.

[21] H. Raether, Surface Plasmons on Smooth and Rough Surfaces and on Gratings, vol. 111 of Springer Tracts in Modern Physics. Springer-Verlag, Berlin, 1988, ISBN 978-0387173634.

[22] H. Raether, Excitation of Plasmons and Interband Transitions by Electrons, vol. 88 of Springer Tracts in Modern Physics. Springer-Verlag, Berlin, 1980, ISBN 978-3540096771. 
[23] F. J. García de Abajo, "Optical excitations in electron microscopy," Rev. Mod. Phys. 82 (2010) 209.

[24] J. Dostálek, J. Homola, S. Jiang, J. Ladd, S. Löfås, A. McWhirter, D. G. Myszka, I. Navratilova, M. Piliarik, J. Štěpánek, A. Taylor, and H. Vaisocherová, Surface Plasmon Resonance Based Sensors. Springer Series on Chemical Sensors and Biosensors. Springer-Verlag, Berlin Heidelberg, 2006, ISBN 978-3642070464.

[25] R. W. Wood, "On a remarkable case of uneven distribution of light in a diffraction grating spectrum," Philosophical Magazine 4 no. 21, (1902) 396-402.

[26] L. Rayleigh, "On the Dynamical Theory of Gratings," Proc. R. Soc. Lond. A 79 no. 532, (1907) 399-416.

[27] R. B. Schasfoort and A. J. Tudos, eds., Handbook of Surface Plasmon Resonance. The Royal Society of Chemistry, 2008, ISBN 978-0854042678.

[28] U. Fano, "The Theory of Anomalous Diffraction Gratings and of Quasi-Stationary Waves on Metallic Surfaces (Sommerfeld's Waves)," J. Opt. Soc. Am. 31 (1941) 213-222.

[29] R. Ritchie, "Plasma Losses by Fast Electrons in Thin Films," Phys. Rev. 106 no. $5,(1957) 874$.

[30] C. J. Powell and J. B. Swan, "Origin of the Characteristic Electron Energy Losses in Magnesium," Phys. Rev. 116 (1959) 81-83.

[31] C. J. Powell and J. B. Swan, "Effect of Oxidation on the Characteristic Loss Spectra of Aluminum and Magnesium," Phys. Rev. 118 (1960) 640-643.

[32] T. Turbadar, "Complete Absorption of Light by Thin Metal Films," Proc. Phys. Soc. 73 no. 1, (1959) 40.

[33] A. Otto, "Excitation of nonradiative surface plasma waves in silver by the method of frustrated total reflection," Zeits. Phys. 216 no. 4, (1968) 398-410.

[34] E. Kretschmann and H. Raether, "Radiative decay of nonradiative surface plasmons excited by light," Z. Naturforsch. A 23 (1968) 2135-2136.

[35] F. H. K. Koppens, D. E. Chang, and F. J. García de Abajo, "Graphene plasmonics: A platform for strong light-matter interaction," cond-mat.mes-hall (2011) 1-39. 
[36] A. Vakil and N. Engheta, "One-Atom-Thick IR Metamaterials and Transformation Optics Using Graphene,".

[37] P. Drude, "Zur Elektronentheorie der Metalle," Ann. Phys. 306 no. 3, (1900) $566-613$.

[38] P. Drude, "Zur Elektronentheorie der Metalle; II. Teil. Galvanomagnetische und thermomagnetische Effecte," Ann. Phys. 3 (1900) 369-402.

[39] N. W. Ashcroft and N. D. Mermin, Festkörperphysik. Oldenbourg, München, 2007, ISBN 978-3-48658273-4.

[40] F. Ladstädter, U. Hohenester, P. Puschnig, and C. Ambrosch-Draxl, "First-principles caculation of hot-electron scatterings in metals," Phys. Rev. B 70 (2004) 235125.

[41] P. B. Johnson and R. W. Christy, "Optical Constants of the Noble Metals," Phys. Rev. B 6 (1972) 12.

[42] C. Sönnichsen, Plasmons in metal nanostructures. PhD thesis, Fakultät für Physik der Ludwig-Maximilians-Universität München, 2001.

[43] E. D. Palik, ed., Handbook of Optical Constants of Solids. Academic Press, New York, 1985, ISBN 978-0125444200.

[44] U. Kreibig and M. Vollmer, Optical Properties of Metal Clusters, vol. 25 of Springer Series in Material Science. Springer, Berlin, 1995, ISBN 978-3-540-57836-9.

[45] S. A. Maier, Plasmonics: Fundamentals and Applications. Springer, Berlin, 2007, ISBN 978-0-387-33150-8.

[46] B. Lamprecht, Ultrafast plasmon dynamics in metal nanoparticles. PhD thesis, Institut für Physik, Karl-Franzens-Universität Graz, 2000.

[47] M. I. Stockman, "Nanoplasmonics: The physics behind the applications," Physics Today 64 (2011) 39-44.

[48] M. I. Stockman, "Dark-hot resonances," Nat. 467 (2010) 541-542.

[49] U. Leonhardt, "Optical metamaterials: Invisibility cup," Nat. Phot. 1 (2007) 207-208.

[50] H. Tait, ed., Five Thousand Years of Glass. University of Pennsylvania Press, 2004, revised edition, ISBN 978-0-8122-1888-6. 
[51] J. Perèz-Juste, I. Pastoriza-Santos, L. M. Liz-Marzàn, and P. Mulvaney, "Gold nanorods: Synthesis, characterization and applications," Coord. Chem. Rev. 249 (2005) 1870-1901.

[52] S. K. Mitra, N. Dass, , and N. C. Varshneya, "Temperature Dependence of the Refractive Index of Water," J. Chem. Phys. 57 (1972) 1798.

[53] J. Becker, Plasmons as Sensors. PhD thesis, Fachbereich Chemie, Pharmazie und Geowissenschaften der Johannes-Gutenberg Universität Mainz, 2010.

[54] A. Ohlinger, S. Nedev, A. A. Lutich, and F. J., "Optothermal Escape of Plasmonically Coupled Silver Nanoparticles from a Three-Dimensional Optical Trap," Nano Lett. 11 (2010) 1770.

[55] S. Gerber, F. Reil, U. Hohenester, T. Schlagenhaufen, J. R. Krenn, and A. Leitner, "Tailoring light emission properties of fluorophores by coupling to resonance-tuned metallic nanostructures," Phys. Rev. B 75 (2007) 073404.

[56] U. Hohenester and A. Trügler, "Interaction of single molecules with metallic nanoparticles," IEEE Journal of Selected Topics in Quantum Electronics 14 (2008) 1430.

[57] A. Kinkhabwala, Z. Yu, S. Fan, Y. Avlasevich, K. Müllen, and W. E. Moerner, "Large single-molecule fluorescence enhancements produced by a bowtie nanoantenna," Nat. Phot. 3 (2009) 654-657.

[58] M. A. Cooper, "Optical biosensors in drug discovery," Nat. Rev. Drug Discovery 1 (2002) 515-528.

[59] H. A. Atwater and A. Polman, "Plasmonics for improved photovoltaic devices," Nat. Mat. 9 (2010) 205-213.

[60] V. Myroshnychenko, J. Rodríguez-Fernández, I. Pastoriza-Santos, A. M. Funston, C. Novo, P. Mulvaney, L. M. Liz-Marzán, and F. J. G. de Abajo, "Modelling the optical response of gold nanoparticles," Chem. Soc. Rev. $\mathbf{3 7}$ (2008) 1792-1805.

[61] J.-S. Huang, J. Kern, P. Geisler, P. Weinmann, M. Kamp, A. Forchel, P. Biagioni, and B. Hecht, "Mode Imaging and Selection in Strongly Coupled Nanoantennas," Nano Lett. 10 (2010) 2105.

[62] C.-K. Sun, F. Vallée, L. H. Acioli, E. P. Ippen, and J. G. Fujimoto, "Femtosecond-tunable measurement of electron thermalization in gold," Phys. Rev. B 50 (1994) 15337-15348. 
[63] M. Wolf, "Femtosecond dynamics of electronic excitations at metal surfaces," Surf. Scie. 377-379 (1997) 343-349. 首 European Conference on Surface Science.

[64] M. Burresi, D. van Oosten, T. Kampfrath, H. Schoenmaker, R. Heideman, A. Leinse, and L. Kuipers, "Probing the Magnetic Field of Light at Optical Frequencies," Science 326 (2009) 550-553.

[65] H. Giessen and R. Vogelgesang, "Glimpsing the Weak Magnetic Field of Light," Science 23 (2009) 529-530.

[66] J. D. Jackson, Classical Electrodynamics. Wiley, New York, 1962, ISBN 978-0-471-30932-1.

[67] W. Thirring, Lehrbuch der Mathematischen Physik - Band 2 Klassische Feldtheorie. Springer, Wien, New York, 1989, ISBN 978-3211821695.

[68] E. T. Whittaker, A history of the theories of aether and electricity: from the age of Descartes to the close of the nineteenth century. University of California Libraries, 2011 (1910), ISBN 978-1125241103.

[69] C. Cohen-Tannoudji, J. Dupont-Roc, and G. Grynberg, Photons and Atoms Introduction to Quantum Electrodynamics. Wiley-VCH, New York, 1997, ISBN 978-0-471-18433-1.

[70] E. Altewischer, W. P. van Exter, and J. P. Woerdman, "Plasmon-assisted transmission of entangled photons," Nat. 418 (2002) 304-306.

[71] M. O. Scully and M. S. Zubairy, Quantum Optics. Cambridge University Press, UK, 1997, ISBN 978-0521435956.

[72] J. Zuloaga, E. Prodan, and P. Nordlander, "Quantum Description of the Plasmon Resonances of a Nanoparticle Dimer," Nano Lett. 9 (2009) 887-891.

[73] S. J. Plimpton and W. E. Lawton, "A Very Accurate Test of Coulomb's Law of Force Between Charges," Phys. Rev. 50 (1936) 1066-1071.

[74] R. Lakes, "Experimental Limits on the Photon Mass and Cosmic Magnetic Vector Potential," Phys. Rev. Lett. 80 (1998) 1826-1829.

[75] D. L. Mills, Nonlinear Optics: Basic Concepts. Springer, Heidelberg, 1998, ISBN 978-3540541929.

[76] Y. R. Shen, The Principles of Nonlinear Optics. John Wiley \& Sons, New York, 1984, ISBN 978-0-471-43080-3. 
[77] M. I. Stockman, D. J. Bergman, C. Anceau, S. Brasselet, and J. Zyss, "Enhanced Second-Harmonic Generation by Metal Surfaces with Nanoscale Roughness: Nanoscale Dephasing, Depolarization, and Correlations," Phys. Rev. Lett. 92 (2004) 057402.

[78] T. Hanke, G. Krauss, D. Träutlein, B. Wild, R. Bratschitsch, and A. Leitenstorfer, "Efficient Nonlinear Light Emission of Single Gold Optical Antennas Driven by Few-Cycle Near-Infrared Pulses," Phys. Rev. Lett. 103 (2009) 257404.

[79] L. V. Lorenz, "On the identity of the vibrations of light with electrical currents," Phil. Mag. 34 (1867) 287-301.

[80] G. Green, An Essay on the Application of mathematical Analysis to the theories of Electricity and Magnetism. G. Green, Nothingham, 1828. Reprinted in Journ. f. Math. in three parts, digitized version available by Google books.

[81] D. M. Cannell and N. J. Lord, "George Green, Mathematician and Physicist 1793-1841," The Mathematical Gazette 77 no. 478, (1993) 26-51.

[82] F. J. García de Abajo and A. Howie, "Retarded field calculation of electron energy loss in inhomogeneous dielectrics," Phys. Rev. B 65 (2002) 115418.

[83] U. Hohenester and J. Krenn, "Surface plasmon resonances of single and coupled metallic nanoparticles: A boundary integral method approach," Phys. Rev. B 72 (2005) 195429.

[84] U. Hohenester, MATLAB ${ }^{\circledast}$ help for MNPBEM toolbox. Institut für Physik, Karl-Franzens-Universität Graz, 2011.

[85] R. Fuchs, "Theory of the optical properties of ionic crystal cubes," Phys. Rev. $B 11$ (1975) 1732.

[86] I. D. Mayergoyz, Z. Zhang, and G. Miano, "Analysis of Dynamics of Excitation and Dephasing of Plasmon Resonance Modes in Nanoparticles," Phys. Rev. Lett. 98 (2007) 147401.

[87] A. Messiah, Quantum Mechanics. North-Holland, Amsterdam, 1965, ISBN 978-0486409245.

[88] U. Hohenester and A. Trügler, "MNPBEM - A Matlab toolbox for the simulation of plasmonic nanoparticles." to be published at Comp. Phys. Com., 2011. 
[89] R. G. Newton, "Optical Theorem and Beyond," Am. J. Phys. 44 (1976) 639-642.

[90] H. C. van de Hulst, Light Scattering by Small Particles. Dover Publications, New York, 1981, ISBN 978-0486642284.

[91] U. Kreibig, "Hundert Jahre Mie-Theorie," Phys. Unserer Zeit 39 no. 6, (2008) 281-287.

[92] G. Mie, "Beiträge zur Optik trüber Medien, speziell kolloidaler Metalllösungen," Ann. Phys. 330 no. 3, (1908) 377-445.

[93] C. F. Bohren and D. R. Huffman, Absorption and scattering of light by small particles. Wiley-Interscience, 1983, ISBN 978-0471293408.

[94] J. A. Stratton, Electromagnetic Theory. Wiley-IEEE Press, 2007, ISBN 978-0-470-13153-4.

[95] B. T. Draine and P. J. Flatau, "Discrete-dipole approximation for scattering calculations," J. Opt. Soc. Am. A 11 no. 4, (1994) 1491.

[96] B. T. Draine and P. J. Flatau, "User Guide to the Discrete Dipole Approximation Code DDSCAT 7.1," astro-ph.IM (2010) 1-83, arXiv:1002.1505v1.

[97] B. Draine, "The Discrete-Dipole Approximation and its Application to Interstellar Graphite Grains," Astrophys. J. 333 (1988) 848-872.

[98] H. A. Lorentz, The Theory of Electrons and its Applications to the Phenomena of Light and Radiant Heat. Teubner, Leipzig, 1909.

[99] E. M. Purcell and C. R. Pennypacker, "Scattering and absorption of light by nonspherical dielectric grains," Astrophys. J. 186 (1973) 705-714.

[100] A. Sutradhar, G. H. Paulino, and L. J. Gray, Symmetric Galerkin Boundary Element Method. Springer-Verlag, Berlin Heidelberg, 2008, ISBN 978-3-540-68770-2.

[101] M. A. Yurkin and A. G. Hoekstra, "The discrete dipole approximation: An overview and recent developments," J. Quant. Spectrosc. Radiat. Transf. 106 (2007) 558-589. 首 also available on arXiv.

[102] T. Wriedt and U. Comberg, "Comparison of computational scattering methods," J. Quant. Spectrosc. Radiat. Transf. 60 no. 3, (1998) 411-423. 
[103] J. W. Hovenier, K. Lumme, M. I. Mishchenko, N. V. Voshchinnikov, D. W. Mackowski, and J. Rahola, "Computations of scattering matrices of four types of non-spherical particles using diverse methods," J. Quant. Spectrosc. Radiat. Transf. 55 no. 6, (1996) 695-705.

[104] U. Kreibig, "Interface-induced dephasing of Mie plasmon polaritons," App. Phys. B 93 (2008) 79-89.

[105] L. Rayleigh, "On the theory of optical images, with special reference to the microscope," Philos. Mag. 42 (1896) 167-195.

[106] E. Abbe, "Beiträge zur Theorie des Mikroskops und der mikroskopischen Wahrnehmung," Archiv für Mikroskopische Anatomie 9 no. 1, (1873) 413-418.

[107] M. Bosman, V. J. Keast, M. Watanabe, A. I. Maaroof, and M. B. Cortie, "Mapping surface plasmons at the nanometre scale with an electron beam," Nanotechnology 18 (2007) 165505.

[108] J. Nelayah, M. Kociak, O. Stephan, F. J. García de Abajo, M. Tence, L. Henrard, D. Taverna, I. Pastoriza-Santos, L. M. Liz-Martin, and C. Colliex, "Mapping surface plasmons on a single metallic nanoparticle," Nat. Phys. 3 (2007) 348.

[109] R. H. Ritchie and A. Howie, "Inelastic scattering probabilities in scanning transmission electron microscopy," Philos. Mag. A 58 no. 5, (1988) 753-767.

[110] U. Hohenester, H. Ditlbacher, and J. R. Krenn, "Electron-Energy-Loss Spectra of Plasmonic Nanoparticles," Phys. Rev. Lett. 103 (2009) 106801.

[111] F. J. García de Abajo and M. Kociak, "Probing the Photonic Local Density of States with Electron Energy Loss Spectroscopy," Phys. Rev. Lett. 100 (2008) 106804.

[112] J. M. McMahon, S. K. Gray, and G. C. Schatz, "Nonlocal Optical Response of Metal Nanostructures with Arbitrary Shape," Phys. Rev. Lett. 103 (2009) 097403.

[113] M. Z. Maialle, E. A. de Andrada e Silva, and L. J. Sham, "Exciton spin dynamics in quantum wells," Phys. Rev. B 47 (1993) 15776.

[114] P. S. Pershan, "Nonlinear Optical Properties of Solids: Energy Considerations," Phys. Rev. 130 (1963) 919-929. 
[115] E. Adler, "Nonlinear Optical Frequency Polarization in a Dielectric," Phys. Rev. 134 (1964) A728-A733.

[116] M. Lippitz, M. A. van Dijk, and M. Orrit, "Third-Harmonic Generation from Single Gold Nanoparticles," Nano Lett. 5 (2005) 799-802.

[117] B. K. Canfield, H. Husu, J. Laukkanen, B. Bai, M. Kuittinen, J. Turunen, and M. Kauranen, "Local Field Asymmetry Drives Second-Harmonic Generation in Noncentrosymmetric Nanodimers," Nano Lett. 7 (2007) 1251-1255.

[118] J. E. Sipe, D. J. Moss, and H. M. van Driel, "Phenomenological theory of optical second- and third-harmonic generation from cubic centrosymmetric crystals," Phys. Rev. B 35 (1987) 1129-1141.

[119] N. Bloembergen, W. K. Burns, and M. Matsouka, "Reflected third harmonic generated by picosecond laser pulses," Opt. Commun. 1 (1969) 195-198.

[120] U. Gubler and C. Bosshard, "Optical third-harmonic generation of fused silica in gas atmosphere: Absolute value of the third-order nonlinear optical susceptibility $\chi^{(3)}, "$ Phys. Rev. B 61 (2000) 10702-10710.

[121] F. Krausz and E. Wintner, "Atmospheric influences in optical third-harmonic generation experiments," App. Phys. B 49 (1989) 479-483.

[122] P. K. Jain, W. Y. Huang, and M. A. El-Sayed, "On the universal scaling behavior of the distance decay of plasmon coupling in metal nanoparticle pairs: a plasmon ruler equation," Nano Lett. 7 (2007) 2080-2088.

[123] C. Sönnichsen, B. Reinhard, J. Liphardt, and A. Alivisatos, "A molecular ruler based on plasmon coupling of single gold and silver nanoparticles," Nat. Biotechnol. 23 (2005) 741-745.

[124] H. Wang and B. M. Reinhard, "Monitoring Simultaneous Distance and Orientation Changes in Discrete Dimers of DNA Linked Gold Nanoparticles," J. Phys. Chem. C 113 (2009) 11215-11222.

[125] B. M. Reinhard, M. Siu, H. Agarwal, A. P. Alivisatos, and J. Liphardt, "Calibration of Dynamic Molecular Rulers Based on Plasmon Coupling between Gold Nanoparticles," Nano Lett. 5 (2005) 2246-2252.

[126] C. Sönnichsen and A. P. Alivisatos, "Gold Nanorods as Novel Nonbleaching Plasmon-Based Orientation Sensors for Polarized Single-Particle Microscopy," Nano Lett. 5 (2005) 301-304. 
[127] J. M. Bingham, K. A. Willets, N. C. Shah, A. D. Q., and R. P. Van Duyne, "Localized Surface Plasmon Resonance Imaging: Simultaneous Single Nanoparticle Spectroscopy and Diffusional Dynamics," J. Phys. Chem. C 113 (2009) 16839-16842.

[128] S. Pierrat, E. Hartinger, S. Faiss, A. Janshoff, and C. Sönnichsen, "Rotational Dynamics of Laterally Frozen Nanoparticles Specifically Attached to Biomembranes," J. Phys. Chem. C 113 (2009) 11179-11183.

[129] O. Schubert, J. Becker, L. Carbone, Y. Khalavka, T. Provalska, I. Zins, and C. Sönnichsen, "Mapping the Polarization Pattern of Plasmon Modes Reveals Nanoparticle Symmetry," Nano Lett. 8 (2008) 2345-2350.

[130] C. Novo, A. M. Funston, and P. Mulvaney, "Direct observation of chemical reactions on single gold nanocrystals using surface plasmon spectroscopy," Nat. Nanotechnol. 3 (2008) 598-602.

[131] L. Carbone, A. Jakab, Y. Khalavka, and C. Sönnichsen, "Light-Controlled One-Sided Growth of Large Plasmonic Gold Domains on Quantum Rods Observed on the Single Particle Level," Nano Lett. 9 (2009) 3710-3714.

[132] J. Homola, S. S. Yee, and G. Gauglitz, "Surface plasmon resonance sensors: review," Sens. Actuat. B Chem. 54 (1999) 3-15.

[133] C. Sönnichsen, S. Geier, N. E. Hecker, G. von Plessen, J. Feldmann, H. Ditlbacher, B. Lamprecht, J. R. Krenn, F. R. Aussenegg, V. Z. H. Chan, J. P. Spatz, and M. Moller, "Spectroscopy of single metallic nanoparticles using total internal reflection microscopy," Appl. Phys. Lett. 77 (2000) $2949-2951$.

[134] G. Raschke, S. Kowarik, T. Franzl, C. Sönnichsen, T. A. Klar, J. Feldmann, A. Nichtl, and K. Kürzinger, "Biomolecular Recognition Based on Single Gold Nanoparticle Light Scattering," Nano Lett. 3 (2003) 935-938.

[135] C. Sönnichsen, T. Franzl, T. Wilk, G. von Plessen, and J. Feldmann, "Drastic Reduction of Plasmon Damping in Gold Nanorods," Phys. Rev. Lett. $8 \mathbf{8}$ (2002) 077402.

[136] J. Becker, O. Schubert, and C. Sönnichsen, "Gold Nanoparticle Growth Monitored in situ Using a Novel Fast Optical Single-Particle Spectroscopy Method," Nano Lett. 7 (2007) 1664-1669.

[137] A. D. McFarland and R. P. Van Duyne, "Single Silver Nanoparticles as Real-Time Optical Sensors with Zeptomole Sensitivity," Nano Lett. 3 (2003) 1057-1062. 
[138] C. L. Baciu, J. Becker, A. Janshoff, and C. Sönnichsen, "Protein-Membrane Interaction Probed by Single Plasmonic Nanoparticles," Nano Lett. 8 (2008) $1724-1728$.

[139] K. S. Lee and M. A. El-Sayed, "Gold and Silver Nanoparticles in Sensing and Imaging: Sensitivity of Plasmon Response to Size, Shape, and Metal Composition," J. Phys. Chem. B 110 (2006) 19220-19225.

[140] Y. Khalavka, J. Becker, and C. Sönnichsen, "Synthesis of Rod-Shaped Gold Nanorattles with Improved Plasmon Sensitivity and Catalytic Activity," J. Am. Chem. Soc. 131 (2009) 1871-1875.

[141] N. Liu, T. Weiss, M. Mesch, L. Langguth, U. Eigenthaler, M. Hirscher, C. Sönnichsen, and H. Giessen, "Planar Metamaterial Analogue of Electromagnetically Induced Transparency for Plasmonic Sensing," Nano Lett. 10 (2010) 1103-1107.

[142] J. Becker, I. Zins, A. Jakab, Y. Khalavka, O. Schubert, and C. Sönnichsen, "Plasmonic Focusing Reduces Ensemble Linewidth of Silver-Coated Gold Nanorods," Nano Lett. 8 (2008) 1719-1723.

[143] J. Burgin, M. Z. Liu, and P. Guyot-Sionnest, "Dielectric Sensing with Deposited Gold Bipyramids," J. Phys. Chem. C 112 (2008) 19279-19282.

[144] L. J. Sherry, S. H. Chang, G. C. Schatz, R. P. Van Duyne, B. J. Wiley, and Y. N. Xia, "Localized Surface Plasmon Resonance Spectroscopy of Single Silver Nanocubes," Nano Lett. 5 (2005) 2034-2038.

[145] S. W. Prescott and P. Mulvaney, "Gold nanorod extinction spectra," J. Appl. Phys. 99 (2006) 123504.

[146] G. W. Bryant, F. J. García de Abajo, and J. Aizpurua, "Mapping the Plasmon Resonances of Metallic Nanoantennas," Nano Lett. 8 (2008) 631-636.

[147] M. Z. Liu and P. Guyot-Sionnest, "Synthesis and Optical Characterization of Au/Ag Core/Shell Nanorods," J. Phys. Chem. B 108 (2004) 5882-5888.

[148] J. A. Osborn, "Demagnetizing Factors of the General Ellipsoid," Phys. Rev. 67 (1945) 351-357.

[149] A. Lambrecht, I. Pirozhenko, L. Duraffourg, and P. Andreucci, "The Casimir effect for silicon and gold slabs," EPL 77 (2007) 44006. 
[150] M. Cao, M. Wang, and N. Gu, "Optimized Surface Plasmon Resonance Sensitivity of Gold Nanoboxes for Sensing Applications," J. Phys. Chem. C 113 (2009) 1217-1221.

[151] B. Nikoobakht and M. A. El-Sayed, "Preparation and Growth Mechanism of Gold Nanorods (NRs) Using Seed-Mediated Growth Method," Chem. Mater. 15 (2003) 1957-1962.

[152] K. M. Mayer, F. Hao, S. Lee, P. Nordlander, and J. H. Hafner, "A single molecule immunoassay by localized surface plasmon resonance," Nanotechnol. 21 (2010) 255503.

[153] C. L. Wu and Q. H. Xu, "Stable and Functionable Mesoporous Silica-Coated Gold Nanorods as Sensitive Localized Surface Plasmon Resonance (LSPR) Nanosensors," Langmuir 25 (2009) 9441-9446.

[154] M. A. Otte, B. Sepulveda, W. H. Ni, J. P. Juste, L. M. Liz-Marzan, and L. M. Lechuga, "Identification of the Optimal Spectral Region for Plasmonic and Nanoplasmonic Sensing," ACS Nano 4 (2010) 349-357.

[155] K. L. Kelly, E. Coronado, L. L. Zhao, and G. C. Schatz, "The Optical Properties of Metal Nanoparticles: The Influence of Size, Shape, and Dielectric Environment," J. Phys. Chem. B 107 (2003) 668-677.

[156] J. Becker, A. Trügler, A. Jakab, U. Hohenester, and C. Sönnichsen, "The optimal aspect ratio of gold nanorods for plasmonic bio-sensing," Plasmonics 5 (2010) 161.

[157] M. A. Mahmoud and M. A. El-Sayed, "Gold Nanoframes: Very High Surface Plasmon Fields and Excellent Near-Infrared Sensors," J. Am. Chem. Soc. 132 (2010) 12704-12710.

[158] L. M. Liz-Marzan, "Tailoring Surface Plasmons through the Morphology and Assembly of Metal Nanoparticles," Langmuir 22 (2006) 32-41.

[159] P. K. Jain and M. A. El-Sayed, "Noble Metal Nanoparticle Pairs: Effect of Medium for Enhanced Nanosensing," Nano Lett. 8 (2008) 4347-4352.

[160] E. Ringe, J. M. McMahon, K. Sohn, C. Cobley, Y. N. Xia, J. X. Huang, G. C. Schatz, L. D. Marks, and R. P. Van Duyne, "Unraveling the Effects of Size, Composition, and Substrate on the Localized Surface Plasmon Resonance Frequencies of Gold and Silver Nanocubes: A Systematic Single-Particle Approach," J. Phys. Chem. C 114 (2010) 12511-12516. 
[161] H. Wei, A. Reyes-Coronado, P. Nordlander, J. Aizpurua, and H. X. Xu, "Multipolar Plasmon Resonances in Individual Ag Nanorice," ACS Nano 4 (2010) 2649-2654.

[162] M. D. Malinsky, K. L. Kelly, G. C. Schatz, and R. P. Van Duyne, "Nanosphere lithography: Effect of the substrate on the localized surface plasmon resonance spectrum of silver nanoparticles," J. Phys. Chem. B 105 (2001) 2343-2350.

[163] B. Pietrobon, M. McEachran, and V. Kitaev, "Synthesis of Size-Controlled Faceted Pentagonal Silver Nanorods with Tunable Plasmonic Properties and Self-Assembly of These Nanorods," ACS Nano 3 (2009) 21-26.

[164] A. Unger and M. Kreiter, "Analyzing the Performance of Plasmonic Resonators for Dielectric Sensing," J. Phys. Chem. C 113 (2009) 12243-12251.

[165] J. Sekhon and S. Verma, "Optimal Dimensions of Gold Nanorod for Plasmonic Nanosensors," Plasmonics 6 (2010) 163-169.

[166] Y. Tang and M. Ouyang, "Tailoring Properties and Functionalities of Metal Nanoparticles through Crystallinity Engineering," Nat. Mater. 6 (2007) 754-759.

[167] X. C. Jiang and A. B. Yu, "Silver Nanoplates: A Highly Sensitive Material toward Inorganic Anions," Langmuir 24 (2008) 4300-4309.

[168] N. Cathcart, A. J. Frank, and V. Kitaev, "Silver nanoparticles with planar twinned defects: effect of halides for precise tuning of plasmon resonance maxima from 400 to $>900 \mathrm{~nm}$," Chem. Commun. (2009) 7170-7172.

[169] J. Dorfmüller, R. Vogelgesang, R. T. Weitz, C. Rockstuhl, C. Etrich, T. Pertsch, F. Lederer, and K. Kern, "Fabry-Pérot Resonances in One-Dimensional Plasmonic Nanostructures," Nano Lett. 9 (2009) 2372-2377.

[170] M. Schnell, A. García-Etxarri, A. J. Huber, K. Crozier, J. Aizpurua, and R. Hillenbrand, "Controlling the near-field oscillations of loaded plasmonic nanoantennas," Nat. Phot. 3 (2009) 287-291.

[171] P. Ghenuche, S. Cherukulappurath, T. H. Taminiau, N. F. van Hulst, and R. Quidant, "Spectroscopic Mode Mapping of Resonant Plasmon Nanoantennas," Phys. Rev. Lett. 101 (2008) 116805. 
[172] B. Schaffer, U. Hohenester, A. Trügler, and F. Hofer, "High-resolution surface plasmon imaging of gold nanoparticles by energy-filtered transmission electron microscopy," Phys. Rev. B 79 (2009) 041401(R).

[173] S. Gerber, F. Reil, U. Hohenester, T. Schlagenhaufen, J. R. Krenn, and A. Leitner, "Tailoring light-emission properties of fluorosphores to resonance-tuned metallic nanostructures," Phys. Rev. B 75 (2007) 073404.

[174] P. Anger, P. Bharadwaj, and L. Novotny, "Enhancement and quenching of single-molecule fluorescence," Phys. Rev. Lett. 96 (2006) 113002.

[175] S. Kühn, U. Håkanson, L. Rogobete, and V. Sandoghdar, "Enhancement of Single-Molecule Fluorescence Using a Gold Nanoparticle as an Optical Nanoantenna," Phys. Rev. Lett. 97 (2006) 017402.

[176] B. Lamprecht, G. Schider, R. T. Lechner, H. Ditlbacher, J. R. Krenn, A. Leitner, and F. R. Aussenegg, "Metal Nanoparticle Gratings: Influence of Dipolar Particle Interaction on the Plasmon Resonance," Phys. Rev. Lett. 84 (2000) 4721-4724.

[177] M. I. J. Stich, S. Nagl, O. Wolfbeis, U. Henne, and M. Schaeferling, "A Dual Luminescent Sensor Material for Simultaneous Imaging of Pressure and Temperature on Surfaces," Adv. Funct. Mater. 18 (2008) 1399.

[178] E. Ozbay, "Plasmonics: Merging Photonics and Electronics at Nanoscale Dimensions," Science 311 (2006) 189.

[179] S. Nie and S. R. Emory, "Probing single molecules and single nanoparticles by surface enhanced Raman scattering," Science 275 (1997) 1102.

[180] P. Anger, P. Bharadwaj, and L. Novotny, "Enhancement and Quenching of Single-Molecule Fluorescence," Phys. Rev. Lett. 96 (2006) 113002.

[181] F. J. Garcia-de Abajo and M. Kociak, "Probing the photonic local density of states with electron energy loss spectroscopy," Phys. Rev. Lett. 100 (2008) 106804 .

[182] R. Egerton, "Spatial resolution of nanostructural analysis by electron energy-loss spectroscopy and energy-filtered imaging," Journal of Electron Microscopy 48 (1999) 711.

[183] A. Eggeman, P. J. Dobson, and A. K. Petford-Long, "Optical spectroscopy and energy-filtered transmission electron microscopy of surface plasmons in core-shell nanoparticles," Journal of Applied Physics 101 (2007) 024307. 
[184] W. Grogger, M. Varela, R. Ristau, B. Schaffer, F. Hofer, and K. M. Krishnan, "Energy-filtering transmission electron microscopy on the nanometer length scale," Journal of Electron Spectroscopy and Related Phenomena 143 (2005) 139.

[185] B. Schaffer, G. Kothleitner, and W. Grogger, "EFTEM Spectrum Imaging at High Energy Resolution," Ultramicroscopy 106 (2006) 1129.

[186] G. Kothleitner and F. Hofer, "EELS performance measurements on a new high energy resolution imaging filter," Micron 34 (2003) 211.

[187] J. Turkevich, P. C. Stevenson, and J. Hillier, "The size and the shape factor in colloidal systems," Discussion of the Faraday Society 11 (1951) 9.

[188] G. Kothleitner and B. Schaffer, "Automated EFTEM spectrum image acquisition: Overcoming EELS dynamic range problems," Microscopy and Microanalysis 13 (2007) 156.

[189] U. Hohenester and J. R. Krenn, "Surface plasmon resonances of single and coupled metallic nanoparticles: A boundary integral method approach," Phys. Rev. B 72 (2005) 195429.

[190] F. J. Garcia de Abajo, "Relativistic energy loss and induced photon emission in the interaction of a dielectric sphere with an external electron beam," Phys. Rev. B 59 (1999) 3095.

[191] B. Schaffer, K. Riegler, G. Kothleitner, W. Grogger, and F. Hofer, "Monochromated, spatially resolved electron energy-loss spectroscopic measurements of gold nanoparticles in the plasmon range," Micron 40 (2008) 269-273.

[192] K. Kimoto, G. Kothleitner, W. Grogger, Y. Matsui, and F. Hofer, "Advantages of a monochromator for bandgap measurements using electron energy-loss spectroscopy," Micron 36 (2005) 185.

[193] J. A. Schuller, E. S. Barnard, W. Cai, Y. C. Jun, J. S. White, and M. L. Brongersma, "Plasmonics for extreme light concentration and manipulation," Nat. Mater. 9 (2010) 193.

[194] K. Kneipp, M. Moskovits, and H. Kneipp, eds., Surface-Enhanced Raman Scattering, vol. 103 of Topics in Applied Physics. Springer, Heidelberg, New York, 2006, ISBN 978-3-540-33566-5.

[195] D. E. Chang, A. S. Sørensen, P. R. Hemmer, and M. D. Lukin, "Quantum Optics with Surface Plasmons," Phys. Rev. Lett. 97 (2006) 053002. 
[196] J. B. Pendry, D. Schurig, and D. R. Smith, "Controlling electromagnetic fields," Science $\mathbf{3 1 2}$ (2006) 1780.

[197] J. Rodriguez-Fernandez, A. M. Funston, J. Perez-Juste, R. A. Alvarez-Puebla, L. M. Liz-Marzan, and P. Mulvaney, "The effect of surface roughness on the plasmonic response of individual sub-micron gold spheres," Phys. Chem. Chem. Phys. 11 (2009) 5909.

[198] K.-P. Chen, V. P. Drachev, J. D. Borneman, A. V. Kildishev, and V. M. Shalaev, "Drude Relaxation Rate in Grained Gold Nanoantennas," Nano Lett. 10 (2009) 916.

[199] W. L. Barnes, "Comparing experiment and theory in plasmonics," J. Opt. A. 11 (2009) 114002.

[200] J. S. Huang, V. Callegari, P. Geisler, C. Brüning, J. Kern, J. Prangsma, P. Weinmann, M. Kamp, A. Forchel, P. Biagioni, U.Sennhauser, and B. Hecht, "Atomically flat single-crystalline gold nanostructures for plasmonic nanocircuitry," Nat. Comm. 1 (2010) 150. 首 also on http://arxiv.org/pdf/1004.1961v4arXiv.

[201] A. Hohenau, H. Ditlbacher, B. Lamprecht, J. R. Krenn, A. Leitner, and F. R. Aussenegg, "Electron beam lithography: a helpful tool for nanooptics," Microelec. Engin. 83 (2006) 1464.

[202] C. Pecharromán, J. Pérez-Juste, G. Mata-Osoro, L. M. Liz-Marzán, and P. Mulvaney, "Redshift of surface plasmon modes of small gold rods due to their atomic roughness and end-cap geometry," Phys. Rev. B 77 no. 3, (Jan, 2008) 035418.

[203] K. Crozier, A. Sundaramurthy, G. Kino, and C. Quate, "Optical antennas: resonators for local field enhancement," Journ. Appl. Phys. 94 (2003) 4632.

[204] P. Bharadwaj, B. Deutsch, and L. Novotny, "Optical Antennas," Adv. Opt. Photon. 1 (2009) 438.

[205] M. Danckwerts and L. Novotny, "Optical Frequency Mixing at Coupled Gold Nanoparticles," Phys. Rev. Lett. 98 (2007) 026104.

[206] R. Kim et al, "High-harmonic generation by resonant plasmon field enhancement," Nat. 453 (2008) 757. 
[207] K. D. Ko, A. Kumar, K. H. Fung, R. Ambekar, G. L. Liu, N. X. Fang, and K. C. Toussaint Jr., "Nonlinear Optical Response from Arrays of Au Bowtie Nanoantennas," Nano Lett. 11 (2010) 61.

[208] P. Mühlschlegel, H.-J. Eisler, O. J. F. Martin, B. Hecht, and D. W. Pohl, "Resonant Optical Antennas," Science 308 (2005) 1607.

[209] P. J. Schuck, D. P. Fromm, A. Sundaramurthy, G. S. Kino, and W. E. Moerner, "Improving the Mismatch between Light and Nanoscale Objects with Gold Bowtie Nanoantennas," Phys. Rev. Lett. 94 (2005) 017402.

[210] J. Beermann, S. M. Novikov, T. Sondergaard, A. Boltasseva, and S. I. Bozhevolnyi, "Two-photon mapping of localized field enhancements in thin nanostrip antennas," Opt. Express 16 (2008) 17302.

[211] E. J. R. Vesseur, R. de Waele, M. Kuttge, and A. Polman, "Direct Observation of Plasmonic Modes in Au Nanowires Using High-Resolution Cathodoluminescence Spectroscopy," Nano Lett. 7 (2007) 2843.

[212] J. Merlein, M. Kahl, A. Zuschlag, A. Sell, A. Halm, J. Boneberg, R. Leiderer, A. Leitenstorfer, and R. Bratschitsch, "Nanomechanical control of an optical antenna," Nat. Phot. 2 (2008) 230.

[213] R. e. a. Esteban, "Direct Near-Field Optical Imaging of Higher Order Plasmonic Resonances," Nano Lett. 8 (2008) 3155.

[214] A. C. Jones, R. L. Olmon, S. E. Skrabalak, B. J. Wiley, Y. N. Xia, and M. B. Raschke, "Mid-IR Plasmonics: Near-Field Imaging of Coherent Plasmon Modes of Silver Nanowires," Nano Lett. 9 (2009) 2553.

[215] M. Schnell, A. Garcia-Etxarri, A. J. Huber, K. B. Crozier, A. Borisov, J. Aizpurua, and R. Hillenbrand, "Amplitude- and Phase-Resolved Near-Field Mapping of Infrared Antenna Modes by Transmission-Mode Scattering-Type Near-Field Microscopy," J. Phys. Chem. C 114 (2010) 7341.

[216] A. Sell, G. Krauss, R. Scheu, R. Huber, and A. Leitenstorfer, "8-fs pulses from a compact Er:fiber system: Quantitative modeling and experimental implementation," Opt. Express 17 (2009) 1070.

[217] G. Krauss, S. Lohss, T. Hanke, A. Sell, S. Eggert, R. Huber, and A. Leitenstorfer, "Synthesis of a single cycle of light with compact erbium-doped fibre technology," Nat. Phot. 4 (2010) 33. 
[218] B. Lamprecht, J. R. Krenn, A. Leitner, and F. R. Aussenegg, "Resonant and Off-Resonant Light-Driven Plasmons in Metal Nanoparticles Studied by Femtosecond-Resolution Third-Harmonic Generation," Phys. Rev. Lett. 83 (1999) 4421.

[219] P. Biagioni, J. S. Huang, L. Duo, M. Finazzi, and B. Hecht, "Cross Resonant Optical Antenna," Phys. Rev. Lett. 102 (2009) 256801.

[220] J. Crowell and R. H. Ritchie, "Radiative Decay of Coulomb-Stimulate Plasmons in Spheres," Phys. Rev. 172 (1968) 436-440.

[221] A. Wokaun, J. P. Gordon, and P. Liao, "Radiation Damping in Surface-Enhanced Raman Scattering," Phys. Rev. Lett. 48 (1982) 957.

[222] L. Novotny, "Effective Wavelength Scaling for Optical Antennas," Phys. Rev. Lett. 98 (2007) 266802.

[223] L. Rogobete, F. Kaminski, M. Agio, and V. Sandoghdar, "Design of plasmonic nanoantennae for enhancing spontaneous emission," Opt. Lett. 32 (2007) 1623.

[224] F. Stietz, J. Bosbach, T. Wenzel, T. Vartanyan, A. Goldmann, and F. Träger, "Decay Times of Surface Plasmon Excitation in Metal Nanoparticles by Persistent Spectral Hole Burning," Phys. Rev. Lett. 84 (2000) 5644.

[225] T. Klar, M. Perner, S. Grosse, G. von Plessen, W. Spirkl, and J. Feldmann, "Surface-Plasmon Resonances in Single Metallic Nanoparticles," Phys. Rev. Lett. 80 (1998) 4249.

[226] A. Anderson, K. S. Deryckx, X. G. Xu, G. Steinmeyer, and M. B. Raschke, "Few-Femtosecond Plasmon Dephasing of a Single Metallic Nanostructure from Optical Response Function Reconstruction by Interferometric Frequency Resolved Optical Gating," Nano Lett. 10 (2010) 2519.

[227] F. Sotier, T. Thomay, T. Hanke, J. Korger, S. Mahapatra, A. Frey, K. Brunner, R. Bratschitsch, and A. Leitenstorfer, "Femtosecond few-fermion dynamics and deterministic single-photon gain in a quantum dot," Nat. Phys. 5 (2009) 352.

[228] A. C. Twitchett-Harrison, T. J. V. Yatesa, R. E. Dunin-Borkowski, and P. A. Midgleya, "Quantitative electron holographic tomography for the 3D characterisation of semiconductor device structures," Ultramicroscopy 108 (2008) 1401-1407.

[229] N. Halas, "Plasmonics: An Emerging Field Fostered by Nano Letters," Nano Lett. 10 (2010) 3816-3822. 
[230] P. J. Mohr, B. N. Taylor, and D. B. Newell, "CODATA recommended values of the fundamental physical constants: 2006," Rev. Mod. Phys. 80 (2008) $633-730$.

[231] T. J. Bruno and P. D. N. Svoronos, CRC Handbook of Fundamental Spectroscopic Correlation Charts. CRC Press (Taylor \& Francis Group), 2006, ISBN 978-0849332500.

[232] C. B. Lang and N. Pucker, Mathematische Methoden in der Physik. Spektrum Akademischer Verlag, Heidelberg, Berlin, 2010, ISBN 978-3-8274-1558-5.

[233] M. Abramowitz and I. A. Stegun, Handbook of Mathematical Functions. Dover Publications, New York, 1973, ISBN 978-0486612720.

[234] D. A. Varshalovich, A. N. Moskalev, and V. K. Khersonskii, Quantum Theory of Angular Momentum. World Scientific Publishing, USA, 1988, ISBN 978-9971509965.

[235] J. J. Sakurai, Modern Quantum Mechanics. Addison-Wesley Publishing Company, USA, 1994, ISBN 978-0201539295.

[236] Q. Li, T. Bürgi, and H. Chen, "Preparation of gold nanorods of high quality and high aspect ratio," Journal of Wuhan University of Technology Materials Science Edition 25 (2010) 104-107.

[237] J.-C. Tinguely, The influence of nanometric surface morphology on surface plasmon resonances and surface enhanced effects of metal nanoparticles. $\mathrm{PhD}$ thesis, Institut für Physik, Karl-Franzens-Universität Graz, 2012.

[238] A. R. Siekkinen, J. M. McLellan, J. Chen, and Y. Xia, "Rapid synthesis of small silver nanocubes by mediating polyol reduction with a trace amount of sodium sulfide or sodium hydrosulfide," Chem. Phys. Lett. 432 no. 4-6, (2006) 491-496.

[239] Y. Sun and Y. Xia, "Triangular Nanoplates of Silver: Synthesis, Characterization, and Use as Sacrificial Templates For Generating Triangular Nanorings of Gold," Adv. Mat. 15 no. 9, (2003) 695-699.

[240] D. Koller, Luminescence coupling to dielectric and metallic nanostructures. PhD thesis, Institut für Physik, Karl-Franzens-Universität Graz, 2009. 


\section{List of Equations}

1. Drude-Sommerfeld model of a free electron gas . . . . . . . . . . . . 9

2. Dielectric function of Drude form . . . . . . . . . . . . . . . . 10

3. Plasmon dispersion relation . . . . . . . . . . . . . . . . 14

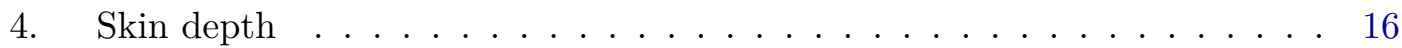

5. Lorentz force in Gaussian units . . . . . . . . . . . . . . . . . . . . . 29

6. Macroscopic Maxwell equations in atomic units . . . . . . . . . . . . . . 33

7. Maxwell's equations in vacuum . . . . . . . . . . . . . . . . 34

8. Linear and nonlinear dipole moment per unit volume . . . . . . . . . . . 37

9. Wave equation for electromagnetic fields . . . . . . . . . . . . . . 39

10. Electromagnetic fields expressed with potentials . . . . . . . . . . . . 40

11. Helmholtz equation for potentials . . . . . . . . . . . . . . . 40

12. Quasistatic Green function . . . . . . . . . . . . . . . . 42

13. Quasistatic surface charge . . . . . . . . . . . . . . . 45

14. Quasistatic surface charge eigenmode expansion . . . . . . . . . . . . 46

15. Retarded Green function and surface derivative . . . . . . . . . . . . . 50

16. Retarded surface charge and current . . . . . . . . . . . . . . 53

17. Clausius-Mossotti relation . . . . . . . . . . . . . 56

18. Cross sections within the quasistatic approximation . . . . . . . 56

19. Scalar wave equation in spherical coordinates . . . . . . . . . . 57

20. Outside Mie solution, scattered wave . . . . . . . . . . . . . . 59

21. Inside Mie solution . . . . . . . . . . . . . . . . . . . . . . . 59

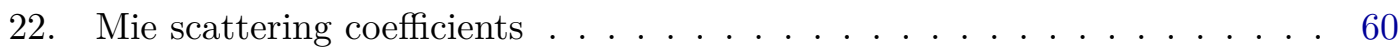


23. Riccati-Bessel functions . . . . . . . . . . . . . . . . . . . 60

24. Mie coefficients for inside field . . . . . . . . . . . . . . . . . 60

25. Polarizations for Mie-Gans solution . . . . . . . . . . . . . . . . . . . 62

26. Discrete Dipole Approximation . . . . . . . . . . . . . . . . . . . . . 64

27. Cross sections with DDA . . . . . . . . . . . . 66

28. Cross sections for retarded simulation . . . . . . . . . . . . 68

29. Electron energy loss probability in terms of Green function . . . . . . . . 80

30. Generation of surface roughness . . . . . . . . . . . . . . . 87

31. Second and third order autocorrelation function . . . . . . . . . . . . . 93

32. Moiré luminescence intensity . . . . . . . . . . . . . . . . . 136

33. Conversion from $\mathrm{eV}$ to $\mathrm{nm}$. . . . . . . . . . . . . . . . . . . . . 184

34. Conversion between FWHM and decay time . . . . . . . . . . . . . . 185 


\section{List of Figures}

2.1. Electronic band structure of gold . . . . . . . . . . . . . . . 11

2.2. Dielectric function for gold and silver . . . . . . . . . . . . . . 12

2.3. Comparison between Palik and Johnson and Christy . . . . . . . . 13

2.4. Evanescent waves at the interface of two media . . . . . . . . . . 14

2.5. Plasmon dispersion relation . . . . . . . . . . . . . 15

2.6. Plasmon excitation by Otto and Kretschmann configuration . . . . . . 16

2.7. Particle plasmon . . . . . . . . . . . . . . . . . 17

2.8. Changing color with nanoparticles in solution . . . . . . . . . 18

2.9. Tuning the plasmon resonance by size and shape . . . . . . . . . . 19

2.10. Tuning the plasmon resonance with $\varepsilon_{b} \ldots \ldots \ldots$

2.11. Fluorophore emission spectrum and sample makeup . . . . . . . . 21

2.12. Nanoantennas as biosensors . . . . . . . . . . . . . . . . 22

2.13. Energy levels and fluorophore decay rates . . . . . . . . . . . . 22

2.14. Nanoantennas as biosensors . . . . . . . . . . . . . . . . . 23

2.15. Example of an optical biosensor . . . . . . . . . . . . . . . . . . 24

2.16. Plasmonic light-trapping geometries for solar cells . . . . . . . . . 25

2.17. Influencing the plasmonic absorption . . . . . . . . . . . . . 26

2.18. Plasmon hybridization . . . . . . . . . . . . . 26

2.19. Plasmon decay processes . . . . . . . . . . . . . . . 27

2.20. Plasmon decay times . . . . . . . . . . . . . . . . . . 28

2.21. Homogeneous linewidth $(\mathrm{FWHM})$ of a plasmon $\ldots . . . . .28$ 
2.22. Detection of electromagnetic fields of optical waves . . . . . . . . . 30

3.1. Scattering spectra of an equilateral and isosceles triangle . . . . . . . . 47

3.2. Surface charge of an equilateral triangle . . . . . . . . . . . . . . 48

3.3. Eigenmodes of an equilateral triangle . . . . . . . . . . . . . . . . 48

3.4. Sum of eigenmodes . . . . . . . . . . . . . . . . . . . . . 49

3.5. Eigenenergies of an equilateral triangle . . . . . . . . . . . . . . . . 49

3.6. Boundary regions for full BEM approach . . . . . . . . . . . . . . . 51

3.7. Surface charge and current of a nanotriangle . . . . . . . . . . . . 53

4.1. Mie modes of different materials . . . . . . . . . . . . . . . 61

4.2. Prolate and oblate ellipsoids . . . . . . . . . . . . . . . . . 62

4.3. Principle of BEM . . . . . . . . . . . . . . . . . . 67

4.4. Electric field of a nanotriangle . . . . . . . . . . . . . . . . 68

4.5. Scattering cross section: BEM, Mie and DDA solution . . . . . . . 70

4.6. Comparison between BEM and DDA . . . . . . . . . . . 71

5.1. Principal scheme of optical Moiré effect . . . . . . . . . . . . . . 79

5.2. First reported plasmon mapping with EELS . . . . . . . . . . . . . . 79

5.3. Principle of EELS interaction . . . . . . . . . . . . . . 80

5.4. Comparison of measured and simulated EFTEM maps . . . . . . . . . 81

5.5. EELS and LDOS maps of coupled particles . . . . . . . . . . . . 82

5.6. Plasmon excitation by electron transmission . . . . . . . . . . . 83

6.1. Smooth and rough rod nanorod surface . . . . . . . . . . . 86

6.2. Generating rough surfaces with respect to an enveloping box . . . . 8 86

6.3. Parameter influence on particle roughness . . . . . . . . . . . . . 87

6.4. Generating realistic particles by contour extraction . . . . . . . . . . 88

7.1. THG intensity for bowtie nanoantenna . . . . . . . . . . . . . . 92 
7.2. THG autocorrelation for nanoantennas . . . . . . . . . . . . . . 93

7.3. Principle of THG measurement . . . . . . . . . . . . . . . . . . . . 94

7.4. Bowtie nanoantenna as single molecule sensor . . . . . . . . . . . . 95

8.1. PAPER: Plasmon resonance shifts and sensitivity . . . . . . . . . 106

8.2. PAPER: FOM to identify the optimal aspect ratio . . . . . . . . 107

8.3. PAPER: Experimental data for the FOM . . . . . . . . . . . . . 108

8.4. PAPER: Quality factor . . . . . . . . . . . . . . . . 110

9.1. PAPER: TEM of silver nanorods with extinction spectra . . . . . . 117

9.2. PAPER: Dark field spectroscopy _ . . . . . . . . . . . . . . . 118

9.3. PAPER: TEM of silver nanorods with extinction spectra . . . . . . . . 120

9.4. PAPER: Cumulative distribution of resonance wavelength shifts . . . 123

9.5. PAPER: Histogram of width and length for silver rods . . . . . . . . . 125

9.6. PAPER: Sensitivity comparison for BEM and Drude model . . . . . . 126

9.7. PAPER: White light scattering spectrum of nanorod . . . . . . . . 126

9.8. PAPER: Spectrum of blue LED lamp . . . . . . . . . . . . . 126

9.9. PAPER: White light scattering spectrum of nanorod . . . . . . . 126

10.1. PAPER: Principles of fluorescence Moiré mapping . . . . . . . . . 131

10.2. PAPER: Fluorescence Moiré mapping of a gold nanoparticle . . . . . . 134

10.3. PAPER: Polarization and wavelength dependence of a nano square . . 135

10.4. PAPER: Polarization and wavelength dependence of a nanorod . . . 137

11.1. PAPER: TEM and EFTEM images . . . . . . . . . . . . . . . . . 142

11.2. PAPER: EELS and STEM EELS spectra . . . . . . . . . . . . 143

11.3. PAPER: Results of BEM simulation . . . . . . . . . . . . . . . 144

12.1. PAPER: Scattering spectra of four rough nanorods . . . . . . . . . . 152

12.2. PAPER: Simulated spectra for nanorods with variing ratios . . . . . . 154 
12.3. PAPER: Comparison of full simulations and perturbation theory . . . 158

13.1. PAPER: Nonlinear emission and damping of antennas . . . . . . . . 161

13.2. PAPER: Spatially resolved third-harmonic emission _ . . . . . . . 163

13.3. PAPER: THG emission intensity of rod nanoantennas . . . . . . . 166

13.4. PAPER: Width dependent damping of rod nanoantennas . . . . . . 167

13.5. PAPER: BEM simulation details . . . . . . . . . . . . . . . . . 169

13.6. PAPER: Simulated scattering spectra of nanoantennas . . . . . . . . . 170

13.7. PAPER: Experimental details for third-harmonic imaging . . . . . . . 171

13.8. PAPER: Third-harmonic intensity of single nanorods . . . . . . . . . 172

A.1. Boundary conditions at different media . . . . . . . . . . . . . . 181

A.2. Energy values of visible spectrum in $\mathrm{nm}$ and $\mathrm{eV}$. . . . . . . . . . 184

A.3. Damped oscillation and Fourier transform . . . . . . . . . . . . . 186

B.1. Electron beam lithography . . . . . . . . . . . . . . . . . . . . 194 


\section{List of Tables}

4.1. CPU time for different BEM simulations . . . . . . . . . . . . . 71

4.2. Performance comparison of BEM, DDA and FDTD . . . . . . . . . 72

5.1. Characteristic length scales in optics . . . . . . . . . . . 75

8.1. PAPER: Quantities describing the quality of plasmon sensors . . . . 108

9.1. PAPER: Size parameters of nanorods . . . . . . . . . . . . . 119

9.2. PAPER: Dimensional characterization of silver nanorods . . . . . . . 125

13.1. PAPER: Antenna dimensions . . . . . . . . . . . . . . . . . 169

C.1. MAtLAB ${ }^{\oplus}$ memory limitations . . . . . . . . . . . . . . . . . 197

C.2. Largest MATLAB ${ }^{\oplus}$ matrix size . . . . . . . . . . . . . . . . . 198 


\section{Index}

\section{A}

Abbe, Ernst .................... 76

Absorption........... see cross section

Absorption broadening...........25

Absorption losses ................ 142

Ampère's law................33, 39

Analytes ................ 101 f., 116

Analytic solutions..............56

Antenna.............. 93, 159-169

bowtie.............. 159, 164

disc-shaped ........... 159, 164

elliptical ................... 159

$\operatorname{rod} \ldots \ldots \ldots \ldots$...... 159, $161 \mathrm{f} ., 165 \mathrm{ff}$.

volume...........160, 165, 167

Antibonding mode.........26, $81 \mathrm{f}$.

Antiquity .................... 18

Aspect ratio . . 101-113, 115-128, 151

optimal..............105, 111

Autocorrelation......91-95, 159-169

Gaussian.............. 85f., 153

\section{B}

Band transition ................. 10

BEM simulation ........44-53, $67 \mathrm{f}$.

Bessel functions ................58f.

Bethe, Hans. . . . . . . . . . . . . . . . . . . .9

Bio-sensing..........4, 19-24, 104

with THG ................ 95

Boltzmann, Ludwig .............. 41

Bonding mode ..........26, 81 f., 95

Bosonic excitation................. 9

Bosonic quasiparticles.............31
Bound charge density ....... 35, 116

Boundary conditions.....43, 45, 52, 155, $181 \mathrm{ff}$.

three dimensional............73

Boundary Element Method......667f.

Boundary integral equations.... 44, 67

Broken symmetry ............... 91

\section{C}

Cancer research ............4, 130

Carbon film ................ $141 \mathrm{f}$, 145

Cassegrain reflector.............. 161

Cathodoluminescence ............. 160

Cavendish, Henry .................. 34

Centrosymmetric material ...... 37, 91

Characterisation of MNPs ........ 124

Characteristic length scales ....... 75

Characteristic peak ratio......... 93

Charge distribution

free vs. bound .............. 35

Charged nanoparticles ............ 73

Chemical interface damping........73

Chemical synthesis...............193

Church windows ................ 18

Classical electrodynamics ..... . 31-53

Classical limit of QED ............ 31

Clausius-Mossotti relation....56, $62 \mathrm{ff}$.

Cold field emission gun ........... 145

Collocation method ............... 68

Color of nanoparticles............. 18

Comparison

BEM vs DDA................71

BEM vs Mie................ 144 
BEM vs Mie vs DDA ........ 70

between methods ............. 70

of CPU time................. 71

of sensors ................. 28

QM vs. Maxwell theory........31

Computer chips

new generation.............. 4

Confinement

of light ............. 159-169

spatial.................... 76

Contour extraction.............. 88

Convergence test

DDA .................. 66

Conversion

between $\varepsilon$ and $n \ldots \ldots \ldots \ldots 11$

between $\mathrm{eV}$ and $\mathrm{nm}$........11, 184

between FWHM and $\tau$.......185

of light ................. 160

Coulomb forces ................. 164

Coulomb's law ................... 34

Coulomb, Charles Augustin de..... 34

Coupled Dipole Approximation ...... see DDA

Coupling to external potential.46, 156

Cross section

absorption ............66, 68

extinction ............66, 68

from Mie-Gans theory . . . . . . 62

scattering ............66, 68

total...................... 55

with BEM .................68 68

with DDA $\ldots \ldots \ldots \ldots \ldots \ldots 66$

with Mie theory $\ldots \ldots \ldots \ldots \ldots 61$

D

D-band absorption...........11f., 47

Damped oscillator ....... see oscillator

Damping ................... 106

intraband ......... see transition

of plasmons ............ radiative......95, 159 f., 165, 167

Dark field microscope . . 105, 117, 151

Data storage ....................4

DDA .................. 63-66

DDSCAT $\ldots \ldots \ldots \ldots \ldots \ldots 64,71$

Decay

channels .................... 27

nonradiative .............. 27

radiative..................27

time ................. $27 \mathrm{f}$.

Decay rate

electromagnetic............. 135

intra-molecular ............ 136

nonradiative ............... 21

of coupled system...........21

of molecule .................. 21

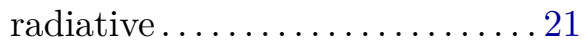

Decoherence time.............. 167

Decoupling of material and structure 45 , 156

Descartes, René..................31

Dichoric glass . ................. 18

Dielectric function ....... $10 \mathrm{f}$, , 13, 16

connection with $n \ldots \ldots \ldots \ldots . .11$

for $\mathrm{Au}$ and $\mathrm{Ag} \ldots \ldots \ldots \ldots . . .12$

from experiment............ 11

of Drude form ........... $9 \mathrm{f}$., 12

Differential geometry.............41

Dimers.......................26

Dipolar mode...134, 138, 151, 157, 164

Dipole moment

linear and nonlinear...........36

per unit volume..............35

Dipole polarization..............64

Direct inversion .................6 65

Dispersion relation............14f., 75

of free electron..............78

Distribution

Fermi-Dirac ................. 9

Maxwell-Boltzmann ...........9 9

DNA strand .................... 3 
Drude model...........9, 108 f., 115

Drude-Sommerfeld model.... . see Drude model

Dye emission

\section{$\mathbf{E}$}

E-beam lithography .... . 85, 151, 194

Earth ............................ 34

EELS ......... 78-83, 130, 139-147 spectra..........83, $141 \mathrm{f} ., 144$

EFTEM..........78, 81, 139-147

Eigenmode expansion ..... 45-49, 89

Eigenmodes .. see plasmon eigenmodes

Eigenvector

left and right..........46, 156

EIT ........................ 104

Electromagnetic waves ........... 40

Electron

beam...........78, 80, 140, 142

charge ................... 9

density ................... 10

gas ...................... 10

gas parameter ............... 10

mass ....................... 9

tunneling .................... 32

Electron-hole pairs ..... 27, 89, 157

Emission

channel modification..........130

rate ..................... 130

Energy dissipation ......... 16, 167

Energy loss .................. 78

probability ................. 144

spectra ........ see EELS spectra

Energy resolution

EFTEM . . . . . . . . . . . . . . . . 141

Energy selecting slit ............ 141

Ensemble average ............... 136

Equilateral triangle ....... see triangle

Evanescent wave........ 14-17, $77 \mathrm{ff}$

Excitation rate ................... 130

selective .................. 77

Exciton..............27, 89, 157

Experimental setup

EELS..................... 141

Moiré mapping ............. 131

optimal sensor ............. 112

particle roughness ........... 151

silver nanorods ....... 119, $123 \mathrm{ff}$.

Exposure time ............ 141, 146

Extinction ......... see cross section

F

Fabrication

of nanoparticles............. 193

top-down ................. 149

Fano, Ugo..................... 7

Far-field .................... 21

Faraday's law ............... 33, 39

Faust ........................ 3

FDTD ......................... 69

Femtosecond dynamics........... 159

Fermi

energy ............... 11, 29

velocity .................... 29

Fermi's golden rule..........21, 130

Fermi-Dirac distribution.............9

Ferroelectrics ................... 36

Few-femtosecond fiber laser ...... 160

Feynman, Richard................. 3

Field

electromagnetic..............33

electromagnetic (via potentials) 40

enhancement ........ see near-field

Figure of merit . . 28, 103 ff., 109 f., 119

for small layers ............. 105

for thin layers...........104, 111

Flow cell ................ 117 f., 125

Fluctuation of atomic fields ....... 31

Fluctuation-dissipation theorem.... 32 
Fluorescence

enhancement...............20

image................... 134

intensity ........... 129, 132

measurement .............. 133

Fluorophore.........20 f., 78, $129 \mathrm{ff}$.

FOM............see figure of merit

Force

electrostatic .............. 34

Lorentz ........ see Lorentz force

Forward scattering amplitude .....55

Fourier

coefficients ..........85, 153

transform . 38, 76, 80, 87, 153, 185

Free charge density .......... 33, 35

Full Maxwell equations...........50

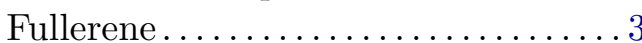

Fundamental mode... . . . . . . . . $164 \mathrm{ff}$.

FWHM........27, 103, 165, 185

\section{G}

Galerkin method .68

Gans solution .. see Mie-Gans solution Gap region......55, 81, 95, 140, 164

GASS.................111, 138

Gauge invariance..............440

Gauss's law ... . . . . . . . . . . . . . . . 33

Gaussian autocorrelation ... . 85 f., 153

Gaussian excitation.............. 165

Gaussian units ............ 5, 33

Glittering metal...............10

Golden rule.... see Fermi's golden rule

Graphene ....................... 9

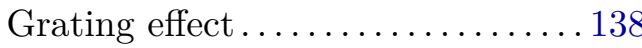

Grating monochromator ......... 161

Green function

dyadic...........21, 32, 136

quasistatic ...........42, 155

retarded .................. 50

singularity ............4 $42 \mathrm{f}$. surface derivative.....46, 50, 156

Green, George .................. 42

\section{$\mathbf{H}$}

Harmonic fields .......... . 33, 93

Harmonic generation

second ..................91

third .......... 92, 159-169

Harmonic oscillator...... see oscillator

Heisenberg's uncertainty principle.... see uncertainty principle

Helmholtz equation ...... 12, 39 f., 51

Hot spot 23, 55, 81, 140, 146, 158, 164

\section{I}

Idealizations .............. 73

Imaging

nonlinear ................ 160

technique ................. 141

third-harmonic . . . . . . . . . . . 159

Induced electric field . . . . . . . . . . . 80

Infrared ................... 10

Interband scattering .... see transition

Interface mode ... see evanescent wave

Interference

destructive............136, 150

Intraband damping ..... see transition

Inversion symmetry ....... . 37, 91

Ion background..............9 $\mathrm{f}$.

Isosceles triangle......... see triangle

Iterative inversion . . . . . . . . . . 65

$\mathbf{J}$

Justification of classical treatment . . 32

$\mathbf{K}$

Kretschmann configuration .... . 8, 16 
$\mathbf{L}$

Langmuir waves................ 7

Laplace equation ............42, 188

Laser

pulse ............... 160, 162

spectrum ................. 162

LDOS .............. 78, 81 f., 134

mapping.................. 78

photonic.................... 140

plasmonic.............130, 132

probing................... 130

Legendre functions ..............58

Liènard-Wiechert potentials . . . 80, 144

Light

absorption in solar cells .......25

bulb....................... 32

concentration ................ 159

cone ...................... 15

elastic scattering............56

harvesting ................. 25

spectrum ................. 184

trapping .................. 25

Light-matter interaction time ...... 91

Linear and nonlinear optics ... . 35-39

Linearity of Maxwell's equations ... 33

Linewidth ............27, 103, 110

Lithography ... see e-beam lithography

Lord Rayleigh .............. 7, 76

Lorentz force.....................29

Lorentz, Hendrik A. ........ 9, 31, 63

Lorentz-Lorenz relation .............. see

Clausius-Mossotti relation

Lorentzian curve............... 186

Lorenz condition ................. 40

Lorenz gauge ................. 50

Low-energy regime............... 140

Luminescence intensity............136

Lycurgus cup .................. 18
M

Mach-Zehnder interferometer .... 162

Macroscopic field................35

Macroscopic phenomena ........... 32

Magnetic permeabilitysee permeability

Magnetic response..............29f.

Magnification ................... 133

Mapping....... see plasmon mapping

Matlab toolbox .................. 68

Matrix inversion ................ 45

Maxwell's equations................ 33

Maxwell, James C................31

Maxwell-Boltzmann distribution.....9

Measurement of plasmonic LDOS . 130

Metallic glittering............... 10

Metamaterial........4, 10, 30, $149 \mathrm{f}$.

Method of Moments ............ 69

Microscopic response .......... 35, 37

Microscopy label................. 102

Mie

coefficients................60

theory ................... 57

Mie, Gustav ................. 57

Mie-Gans solution ...........57, 61

MNPBEM ..............68, 71

Mode mapping. . see plasmon mapping

Moiré

mapping $\ldots \ldots \ldots \ldots 78,129-138$
pattern $\ldots \ldots \ldots \ldots \ldots 78,130$

Molecular

detector.................. 4

emission rate .............. 130

Molecule

as dipole resonator........... 135

decay..................... 135

Monitoring of surface plasmons ... 141

Motional narrowing..... 85, 89, $149 \mathrm{f}$.

Multiple multipole method ........69

Multiple scattering method ....... 69 
$\mathbf{N}$

NA $\ldots . . . \ldots . .$. see numerical aperture Nanoantennas .......... see antenna

Nanooptics......................75

Nanoparticle

in solution ................. 18

polymer see polymer nanoparticles sensor ............... see sensor synthetisation..........112, 193

Nanorod sensitivity ..........115-128

NAWI Graz..............111, 138

Near-field .................21, 30

enhancement ....... 22 f., 140, 164

Near-infrared..........164, 167, 184

Negative refraction .. see metamaterial

Non-Hermitian matrix ............ 46

Nonlinear

effects .................. 91-95

emission............... 159-169

emission spectroscopy......... 167

Nonlocal interaction . . . . . . . . . 80

Numerical aperture...........75, 161

\section{O}

Ohmic power loss

Optical antennas ........ see antenna

Optical data processing ........... 4

Optical gating

frequency resolved 159

Optical response

linear and nonlinear ...... 35-39

modeling .............. 55-73

Optical theorem ................ 55

Optimal sensor ............ 101-113

Optoelectronics ..................4

Orientation sensing .............. 102

Oscillator

damped ...............9, 186

harmonic ............. 93, 185

Otto configuration............ 8,16
$\mathbf{P}$

Parseval condition .............. 191

Particle

plasmons ................. 17

roughness ... see surface roughness

Particle plasmons

excitation.................... 17

Pauli principle ................. 9

Performance .......... see simulation

Permeability

magnetic..............29, 33

Permittivity $\ldots \ldots \ldots \ldots \ldots \ldots \ldots . \ldots 9$

Perturbation analysis ... 89, 155-158

Phase coherence ................ 27

Phonon scattering................27

Photochemical process ........... 122

Photodetector .................. 136

Photoemission ................... 79

Photon

massive.................... 34

massless ................... 34

Photonic energy..................47

Photovoltaics........... 4, 25, 130

Pixel dwell time ................. 146

Planar interface.................. 13

Plasma model..................... 7

Plasmon.................... 7-30

bulk ..................... 10

damping........... $27 \mathrm{ff}$., $160 \mathrm{f}$.

decay time.... 28, 94, 162, $165 \mathrm{ff}$.

dephasing............27ff., 91

derivation................... 9

dimers ..................... 26

dispersion relation ........... 14

dynamics .................. 29

eigenmodes . 46, 129 f., $133 \mathrm{ff}$., $137 \mathrm{ff}$., $144 \mathrm{ff} ., 156$

electron interaction ........... 79

excitation................... 8

first observation.............. 7 
hybridization .............. 26

imaging .......... 139-147, 159

lifetime..............27 ff., 109

linewidth........... see linewidth

magnetic effects ...........29f.

mapping... .78-83, 129-138, 159

oscillation................... 17

particle ..... see particle plasmons

polariton .................. 17

propagation length............ 16

relaxation.................91

resonance .......... 18, 25, 102

resonance tuning ....... see resonance tuning

response times.............. 159

scattering spectroscopy ....... 102

sensitivity ........... 115, 119

sensor ......... 20-24, 115-128

surface....................17

Plasmonic

applications. .4, 130, 138, 140, 150

mode density ............. 130

sensing .................. $19 \mathrm{f}$

Plasmonics........................ 3

Point array $\ldots \ldots \ldots \ldots \ldots \ldots 63$

Poisson equation.......44 f., 155, 188

Polarizability .................. 56

of a spheroid...............106

tensor .................. $64 \mathrm{ff}$.

Polycrystalline particle..... 85, $150 \mathrm{f}$.

Polymer nanoparticles

dye doped ................ 130

Potential

electromagnetic..............39

electrostatic ................ 35

gauge invariance ............ 40

Liènard-Wiechert . . . . . . . . . . . 80

scalar..............40,42f., 155

vector................40, 50

Poynting vector ................68

Principle of near-field optics ...........75

of plasmonic sensing ......20-24

Prism ......................... 16

Product ansatz.................55

Projector .................... 157

Promises of plasmonics............4 4

Pseudorandom numbers...........87

PtTFPP ................ $131 \mathrm{f}$, 136

Purcell, Edward M............63f.

\section{Q}

QED ......................... 31

QSA .... see quasistatic approximation

Quadrupolar mode...............138

Quality factor ............ 101, $109 \mathrm{ff}$.

Quantum emitter .............. 130

Quantum information processing....4

Quantum mechanical description . $31 \mathrm{f}$.

Quantum nature of plasmons.......31

Quantum optics..............4, 75

Quantum yield..................136

Quasistatic approximation...42, 56, 105, 107, 109, 112, 121, 153

Quenching.................. 132

$\mathbf{R}$

Radiative damping....... see damping

Random potential...............85

Rayleigh

scattering $\ldots \ldots \ldots \ldots \ldots 42,56$

theory .................... 56

Reagents.....................123

Refractive index...............10 f.

connection with $\varepsilon \ldots \ldots \ldots \ldots 11$

sensitivity.............19, 116

Regular height variations ........ 155

Relativistic motion...............80

Relativity....... see special relativity

Resolution

diffraction-limited............76 
fundamental limit............75

increase....................77

limit ..................... $76 \mathrm{f}$.

of an optical microscope ...... 75

Resonance

broadening ..............28

linewidth...........see linewidth shift.......19, $101 \mathrm{ff} ., 110,116 \mathrm{f}$. tuning ........... 18 f., $101 \mathrm{f} ., 116$

wavelength ............... 105

Retardation effects..............42

Reversibility............. see sensing

Riccati-Bessel functions . . . . . . . . . 60

Rippled rod.................. 155

Rod width ................... $165 \mathrm{f}$.

Roman cup ........ see Lycurgus cup

Roughness ..... see surface roughness

\section{S}

Sainte Chapelle................. 18

Scalar potential.................40

Scalar wave equationsee wave equation

Scattering........... see cross section

with impurities ............ 27

Schumann resonances ............ 34

Screening.............10, 32, 118

Self-interaction ............21, 79

\section{SEM}

of bowtie antenna...........92

of MNP arrays..............131

of nanoantennas ............ 162

of rough particle ............ 88

Sensing reversibility.............122

Sensitivity .............. 106, 108

best ................... 111

to dielectric environment.. 19, $102 \mathrm{f}$, 116,153

to shape and size........18, 105

Sensor

plasmonic $18 \mathrm{f}$., 102, 111, 116-128 quality ........... 103 f., 108, 111

Shape factor .............62, 107

Sharp features ................. 86

SHG ........ see harmonic generation

Shift of plasmon resonance..18-29, 102, 104,116

Silver nanorods ............ 115-128

Simulation

accuracy ................. 70

EELS ................... $143 \mathrm{f}$.

ensemble of particles......... 135

limits and inaccuracies........73

Moiré mapping ............. 134

optimal sensor ..............111

particle roughness........151, 155

performance................ 71

silver nanorods.......121, $127 \mathrm{f}$.

third-harmonic maps ......... 164

Single particle microscopy......... 124

Single particle sensitivity ... . 117-128

Single plasmon generation ........ 140

Single-cycle limit...............160

Singularity ....... see Green function

Skin depth.................... 16

Smooth nanorod ................. 158

SNOM .................. 78, 160

Solar cells....................25

Sommerfeld, Arnold................ 9

Spatial resolution

EELS................... 140

Special relativity ................ 40

Spectra

absorption............21, $25 \mathrm{f}$.

extinction............661, 131

photoluminescence...........131

scattering .... 47, 117, 151 ff., 166

Spectral selectivity...............133

Spectroscopy

dark field .................. 160

single particle................112

third-harmonic ............. 160 
Spherical harmonics.............57

Spheroidal particles ...........6 $61 \mathrm{f}$.

Spill-out effect ...................73

Spontaneous emission rate.....78, 129

SPP ................... see plasmon

Stained glass...................18

Standard model of particle physics . 31

STEM ....................... 140

Stochastic height variations... 85 ff., 153, 157

Stokes shift ................. 130

Superposition of fields.............33

Superresolution ................. 129

Surface charge ....43, 45, 51, 68, 155

quasistatic................ 45

retarded .................... 52

Surface plasmon.......... see plasmon

Surface roughness... .85-89, 149-158 generation ............. 85-88

Susceptibility

electric .................. 37

magnetic.................29

nonlinear..............37, 94

of gold ....................94

tensor ................. 36,38

Synthesis

of nanoparticles........... $193 \mathrm{f}$.

of nanorods ..........116f., 124

$\mathbf{T}$

Taylor series .................. 36

TE modes ...................... 13

Temperature dependent $n \ldots \ldots \ldots 20$

THG........ see harmonic generation

Thin-film solar cell...... see solar cells

Time delay between pulses .... 93, 162

Timescale

femtosecond ...... 92, 159 ff., 168

ultrafast ............ 91, 160

TM modes ................... $13 \mathrm{f}$.
Total reflection..................16

Transition

interband ......... 12, 145, 167

intra- and interband .........27

intraband................... 109

Triangle

eigenmodes ............... 48

equilateral .............. $47 \mathrm{f}$.

isosceles ................. 48

polarization...............47

surface charge ............. 48

Tuning .......... see resonance tuning

Two-photon photoluminsecence ... 160

$\mathrm{U}$

Ultrafast plasmon dynamics.......91

Ultrafast response ............. 159

Ultraviolet..............10, 21, 184

Uncertainty principle.......... $75 \mathrm{f}$.

V

Vacuum deposition .............. 150

Vector harmonics...............57 f.

Vector potential

cosmic magnetic ............. 34

electromagnetic .........40, 50

Visible spectrum.............. 184

\section{W}

Wave equation

for electromagnetic fields ... 12, 39

for potentials ............... 40

scalar...................... 57

Wave vector at interface ......... 14

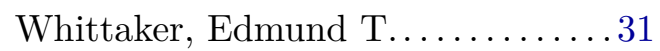

Wood, Robert W................. 7

\section{Z}

Zero loss peak............ 140-146

Zero-frequency limit ........... 64 